\title{
Electrochemical Study of Corrosion Behavior of Stellite 6 Alloy and 17-4PH Stainless Steel in Amine Solutions
}

by

Yi Li

A thesis submitted to the Faculty of Graduate and Postdoctoral Affairs in partial fulfillment of the requirements for the degree of

Master of Applied Science

in

Materials Engineering

Carleton University

Ottawa, Ontario

(C) 2017, Yi Li 


\section{Abstract}

Stellite 6 alloy has been popularly employed as the material for the seat face of various control valves due to its excellent combined mechanical, corrosion, wear, and high temperature properties. However, a suggestion was made in an article that Stellite 6 alloy should be avoided in the amine containing feed water service of power plants, and Stellite 6 alloy should be replaced by stainless steels, such as $17-4 \mathrm{PH}$ stainless steel. To provide the power generation industry with scientific evidence, the corrosion behavior of Stellite 6 alloy and $17-4 \mathrm{PH}$ stainless steel is studied in morpholine solution, cyclohexylamine solution and sodium hydroxide solution at the same $\mathrm{pH}$ around 9.5 using electrochemical test methods such as potentiodynamic polarization, cyclic polarization, and electrochemical impedance spectroscopy (EIS) in this research. The microstructures of the tested materials and corroded surfaces are analyzed using scanning electron microscopy (SEM) with energy dispersive X-ray (EDX) spectroscopy. The experimental results show that Stellite 6 alloy and 17-4PH stainless steel are generally resistant to corrosion in amine solutions by forming a Cr-rich oxide films on their surfaces, but they can be corroded when the oxide film is broken, leading to damage of the surfaces and the removal of the materials. The oxide film of $17-4 \mathrm{PH}$ stainless steel is more resistant to electron transferring than that

of Stellite 6 alloy, thus has better resistance to corrosion. However, the oxide film of 17-4PH stainless steel has less durability and it works well only within a certain potential range. Otherwise, Stellite 6 alloy has better overall corrosion and pitting corrosion resistance than 17-4PH stainless steel in amine environments. 


\section{Acknowledgements}

It is my great pleasure to take this opportunity to thank many people who made this thesis possible.

First, I would like to thank my supervisor, Professor Rong Liu, for her excellent guidance and patience during my research and study at Carleton University. Her professional attitudes toward science and engineering have inspired me on the independent work.

Second, I would like to sincerely thank Professor Glenn McRae for his insightful and knowledgeable advice on the corrosion experiments.

In addition, I would like to thank Mathew Yao, Rachael Collier in assisting me with the sample preparation and providing deep knowledge about Stellite alloys.

Moreover, I would like to thank Dr. Jianqun Wang for his help in SEM/EDX.

I would also like to thank Xiaozhou Zhang, Kun Zhuang, Fu Liu for their sincere advice on writing skills.

Lastly, I would like to thank my parents for their supports morally and financially, and my wife, Mengyang Zha, for her patience and great support during my thesis writing. 


\section{Table of Contents}

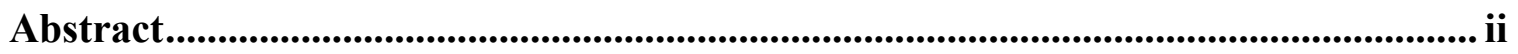

Acknowledgements ....................................................................................................... iii

Table of Contents ............................................................................................. iv

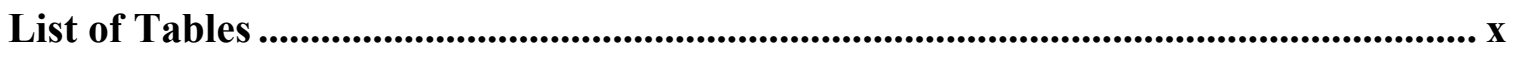

List of Figures........................................................................................................................... xii

List of Appendices ....................................................................................................... $\mathrm{xxi}$

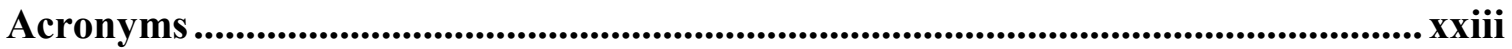

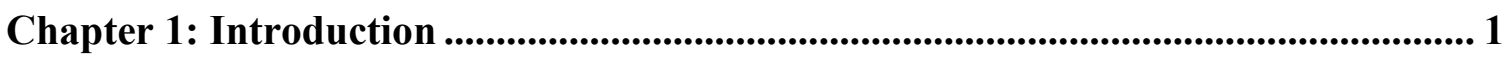

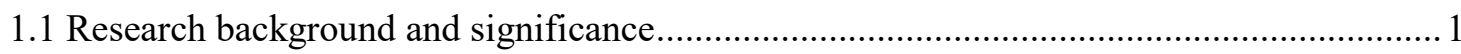

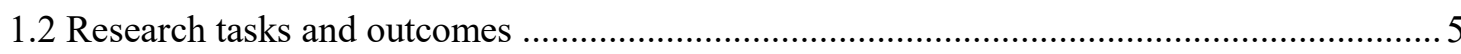

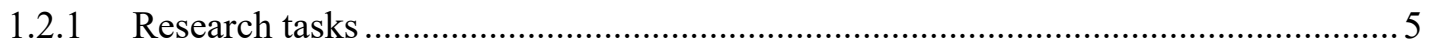

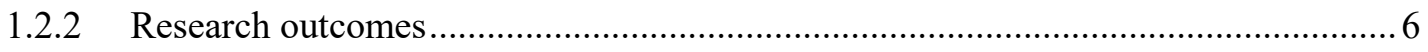

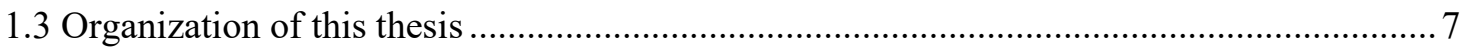

Chapter 2: Literature Review ................................................................................... 10

2.1 Boiling water systems in power generation plants ................................................... 10

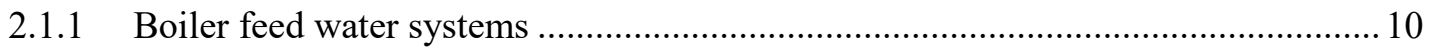

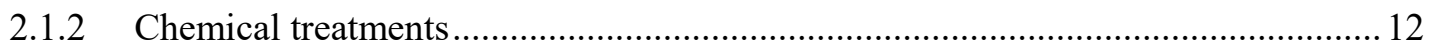

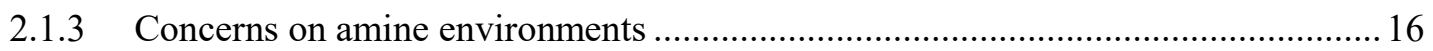

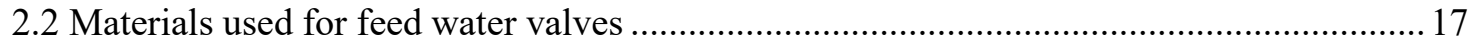

2.2.1 Common materials used for feed water valves .................................................. 17 


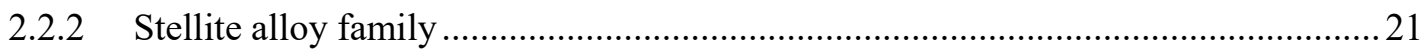

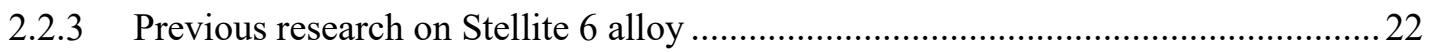

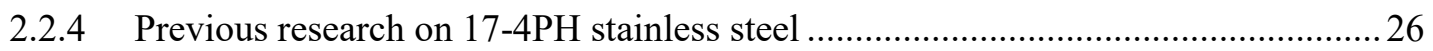

2.2.5 Summary of previous research on Stellite 6 alloy and 17-4PH stainless steel......... 30

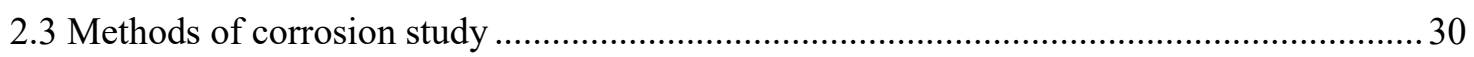

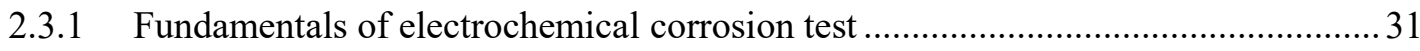

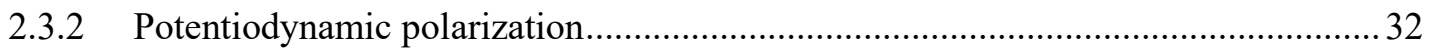

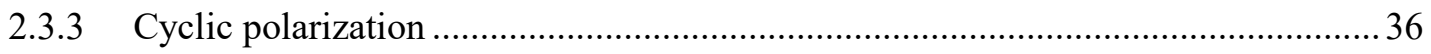

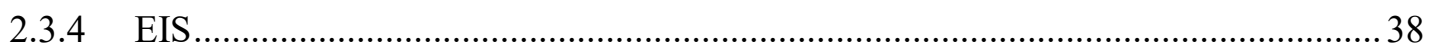

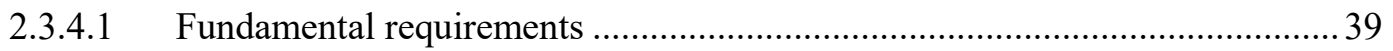

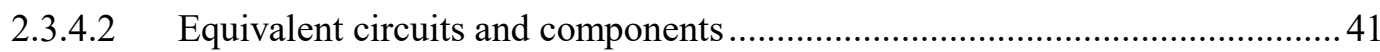

2.3.5 Corrosion studies of metals using electrochemical methods................................. 44

Chapter 3: Electrochemical Corrosion Experiments .............................................. 48

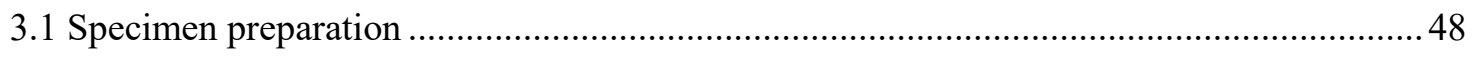

3.1.1 Chemical compositions and microstructure ........................................................ 48

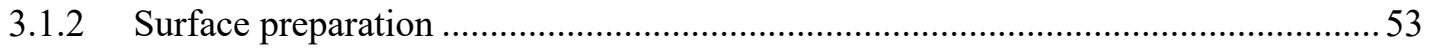

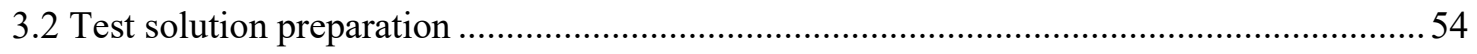

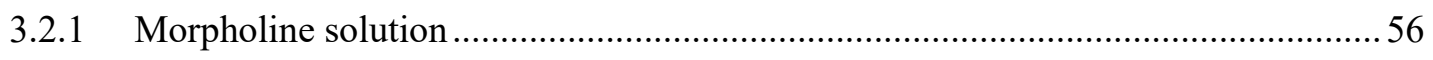

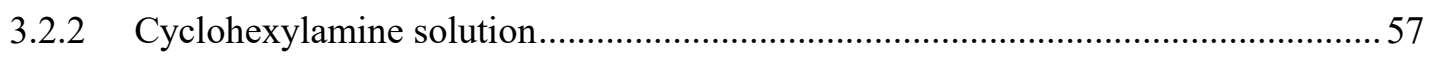

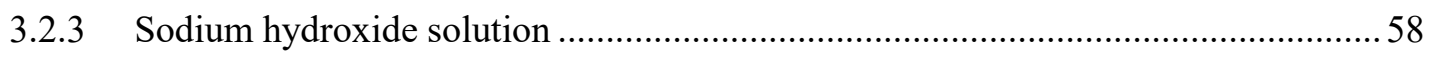

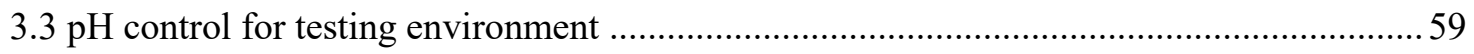




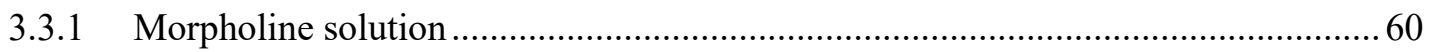

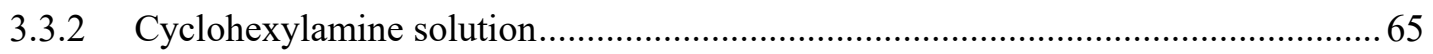

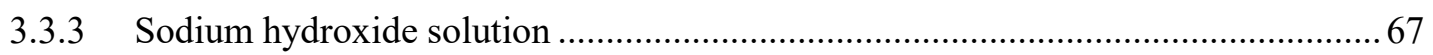

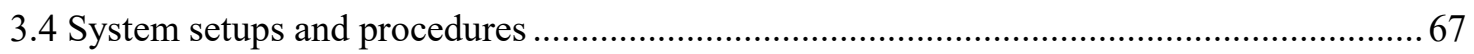

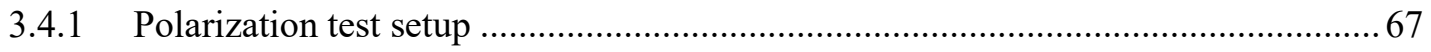

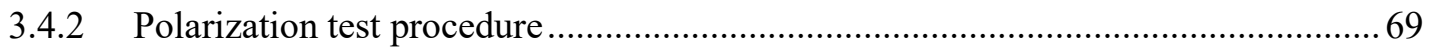

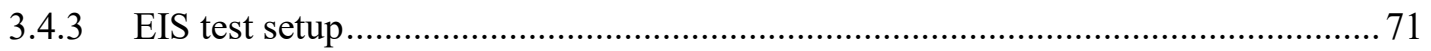

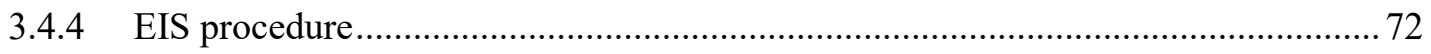

Chapter 4: Experimental Results and Analyses............................................................... 74

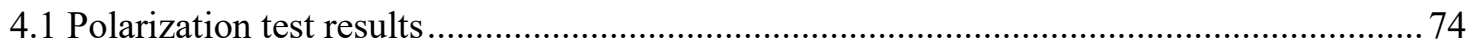

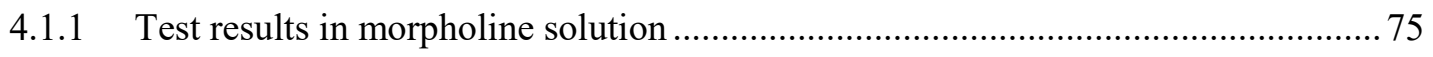

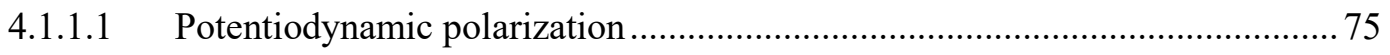

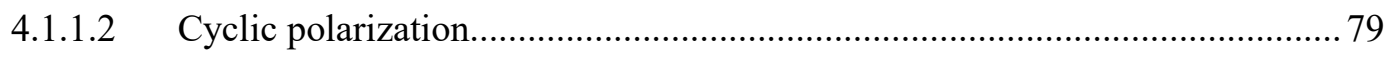

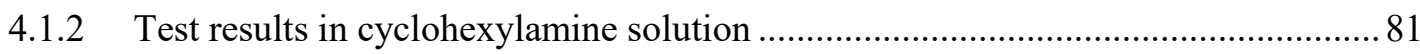

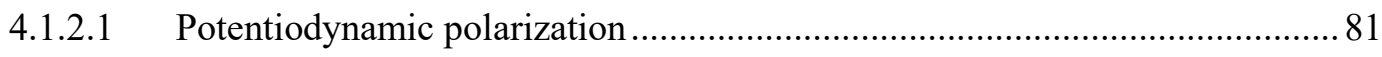

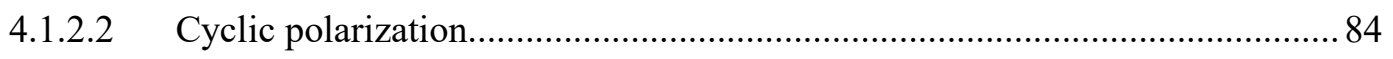

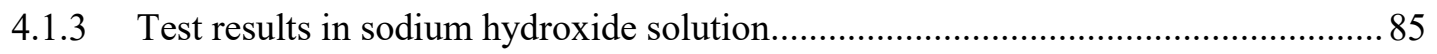

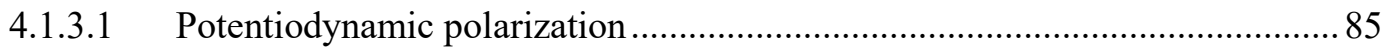

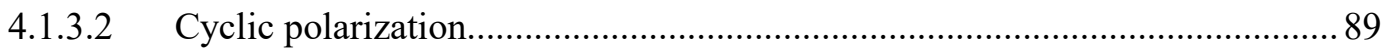

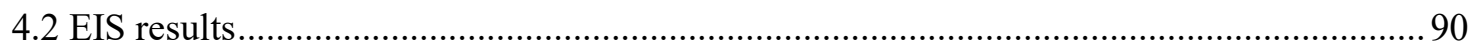

4.2.1 Test results in morpholine solution .............................................................. 91 


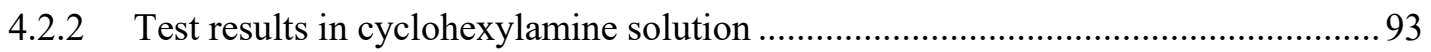

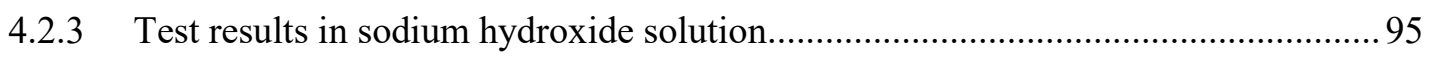

Chapter 5: Failure Tests and Analyses ................................................................................ 98

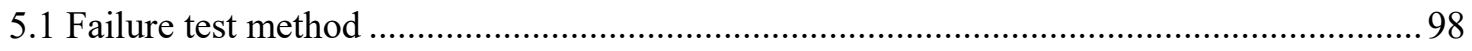

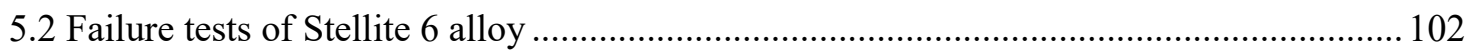

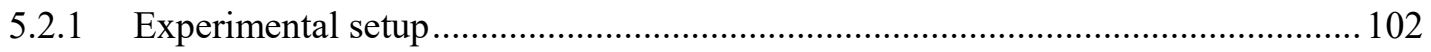

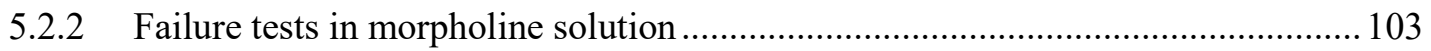

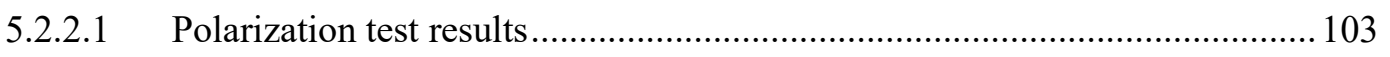

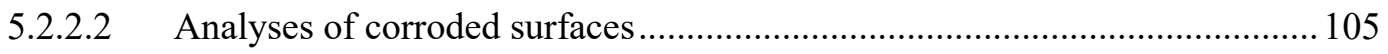

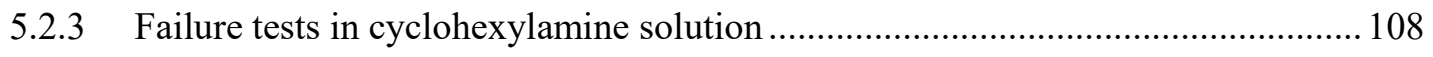

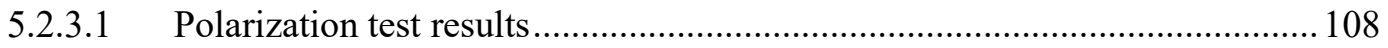

5.2.3.2 Analyses of corroded surfaces ................................................................. 110

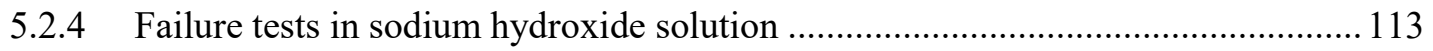

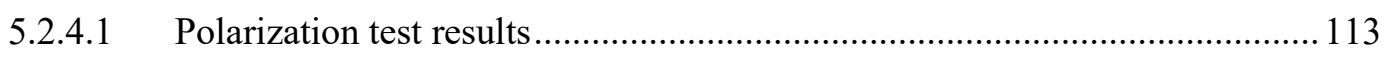

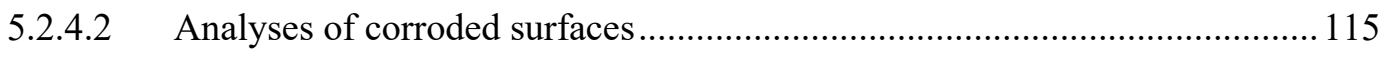

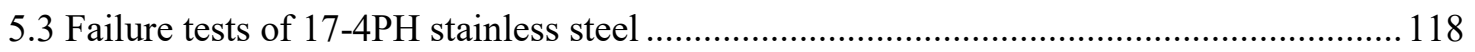

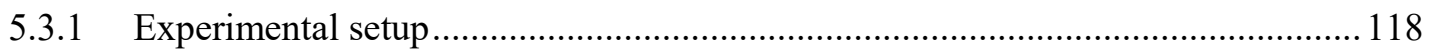

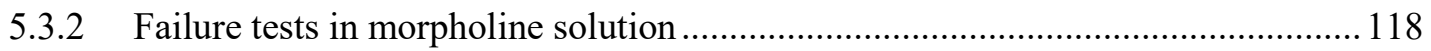

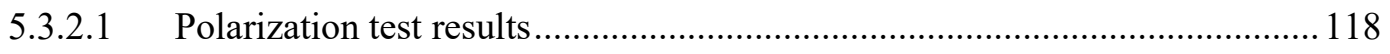

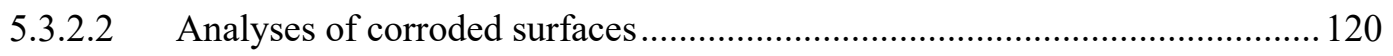

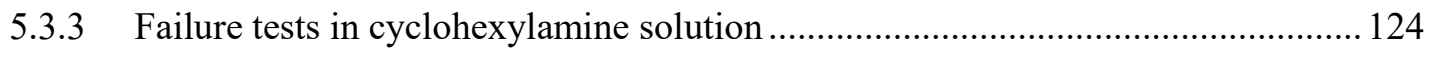




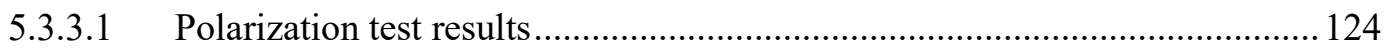

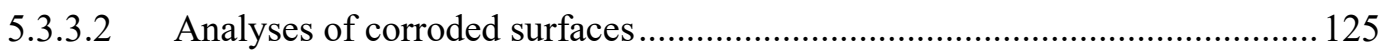

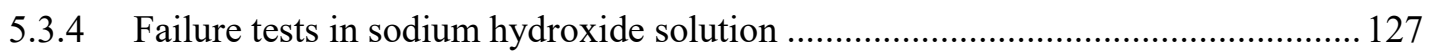

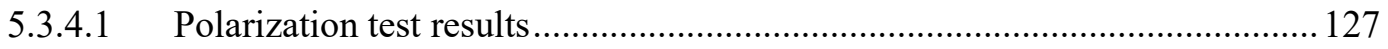

5.3.4.2 Analyses of corroded surfaces ................................................................ 129

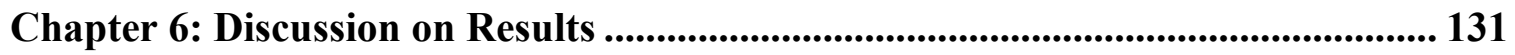

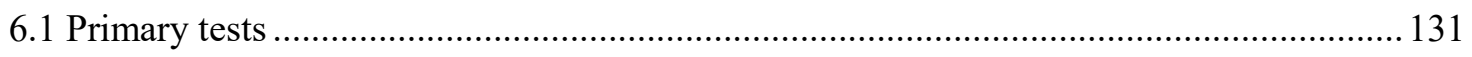

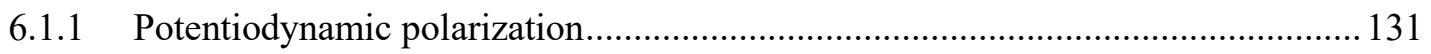

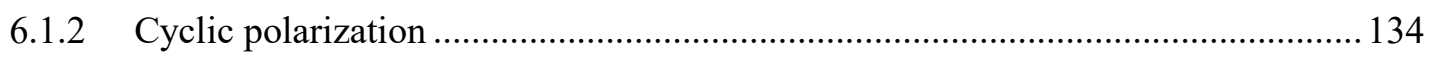

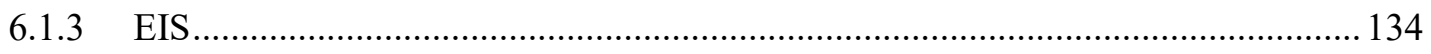

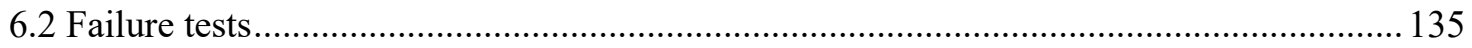

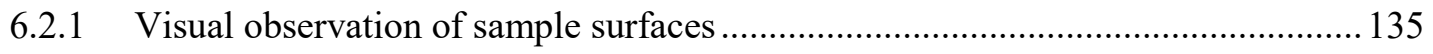

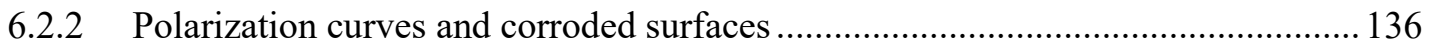

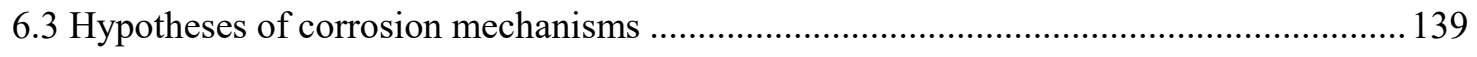

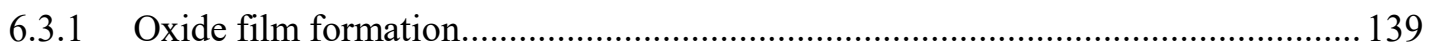

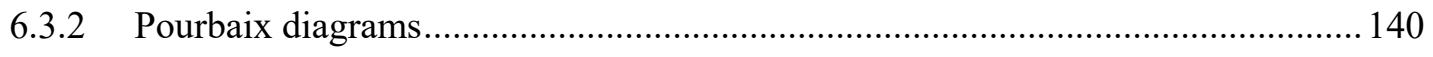

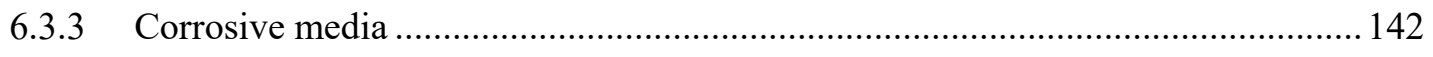

Chapter 7: Conclusions and Future Work ........................................................................ 145

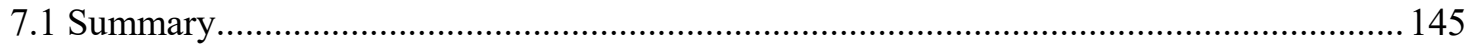

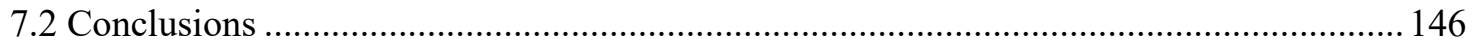

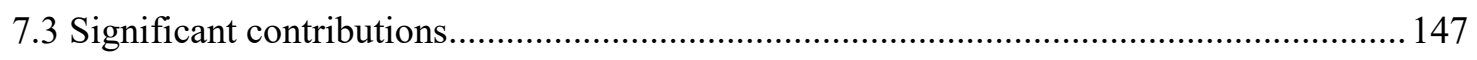


7.4 Future work

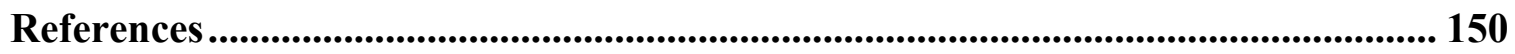

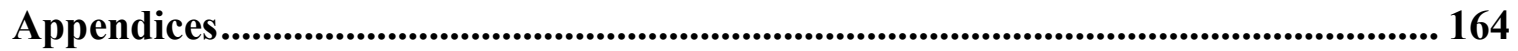




\section{List of Tables}

Table 2-1 Problems caused by impurities in water [16] ................................................ 14

Table 2-2 ASME suggested water chemistry limits for industrial watertube, high duty,

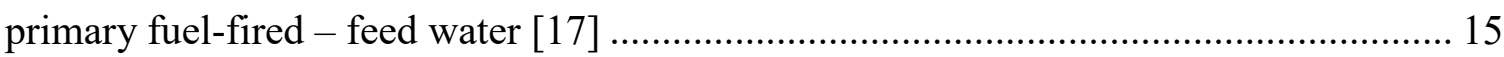

Table 2-3 Boiler feed water treatments for once-through boilers [19] ............................ 16

Table 2-4 Typical chemical compositions (in weight), mechanical properties, and PREN of cast stainless steels used for pumps and valves [21] ............................................. 18

Table 2-5 Relative erosion/abrasion resistance of various alloys [21] ........................... 19

Table 2-6 Common usage of cast stainless steels [21] …………………………….... 20

Table 2-7 Compositions (wt\%, Co in balance) of various Stellite alloys [25] ................ 22

Table 3-1 Chemical compositions of Stellite 6 alloy and 17-4PH stainless steel (wt\%) [40]

Table 3-2 The $\mathrm{pH}$ exploration data of morpholine solution for $2 \mathrm{~h}$................................. 62

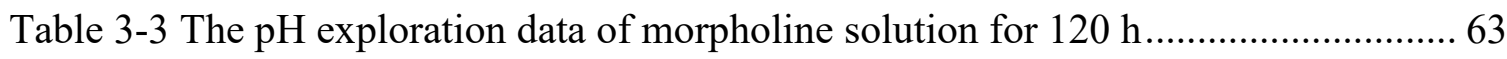

Table 3-4 The pH variation of morpholine solution ........................................................... 64

Table 3-5 The pH exploration data of cyclohexylamine solution for $48 \mathrm{~h} . . . \ldots \ldots \ldots \ldots \ldots \ldots . . . . . . .66$

Table 3-6 The pH variation of cyclohexylamine solution ................................................ 66

Table 4-1 Summary of polarization test results of Stellite 6 alloy and 17-4PH stainless steel

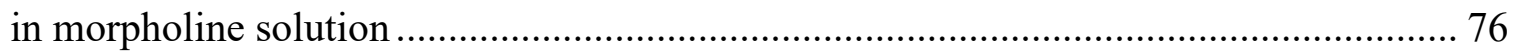

Table 4-2 Summary of polarization test results of Stellite 6 alloy and 17-4PH stainless steel

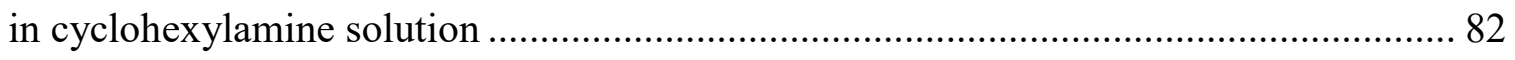


Table 4-3 Summary of polarization test results of Stellite 6 alloy and 17-4PH stainless steel

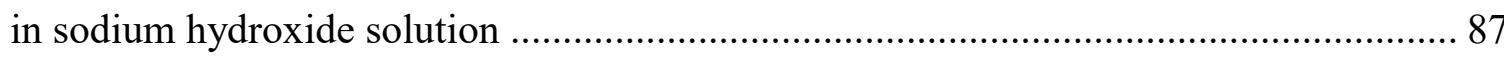

Table 4-4 Electrochemical corrosion parameters fitted from the equivalent circuit in

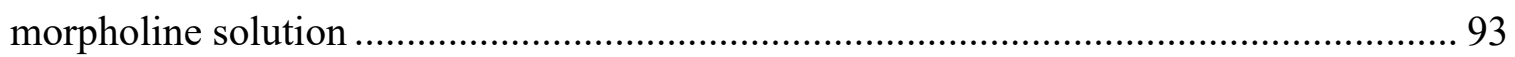

Table 4-5 Electrochemical corrosion parameters fitted from the equivalent circuit in

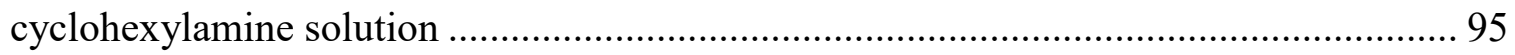

Table 4-6 Electrochemical corrosion parameters fitted from the equivalent circuit in

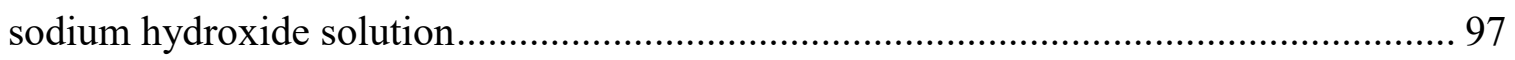
Table 6-1 The slopes of divided phases of polarization curves for Stellite 6 alloy and

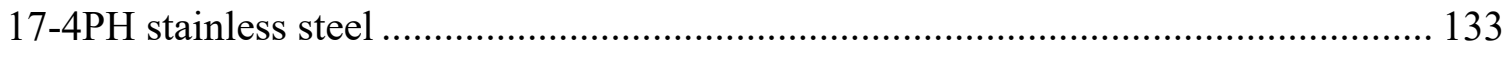

Table 6-2 Average polarization parameters for Stellite 6 alloy and 17-4PH stainless steel 133

Table 6-3 Average charge transfer resistances of Stellite 6 alloy and 17-4PH stainless steel 135 


\section{List of Figures}

Figure 1-1 Erosion/corrosion damage of the valve seat [4] .............................................. 2

Figure 1-2 Interface of Stellite 6 alloy showing cratered face [4] f.................................. 2

Figure 1-3 Schematic illustration of erosion/corrosion attack [5] .................................. 3

Figure 2-1 Typical thermal power plant system [11]................................................ 10

Figure 2-2 Water systems of thermal power plants [12]............................................ 11

Figure 2-3 Schematic of basic boiler system [16] ………........................................... 14

Figure 2-4 Three-terminal electrochemical cell [63] ................................................... 32

Figure 2-5 Typical polarization curves: (a) anodic polarization curve; (b) parameters related to polarization curve (c) Tafel slope [64]....................................................... 35

Figure 2-6 Cyclic polarization curves tested in $\mathrm{pH} 1$ sulfuric acid solution with 10,000 ppm chlorine, $1000 \mathrm{ppm}$ fluorine, and $5000 \mathrm{ppm}$ manganese at $50^{\circ} \mathrm{C}$ for: (a) Alloy G-3; (b) Type

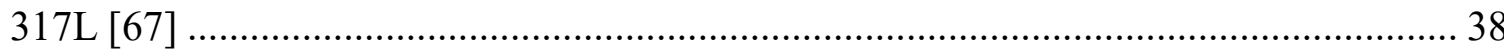

Figure 2-7 Structures used to simulate the electrode process: (a) steady

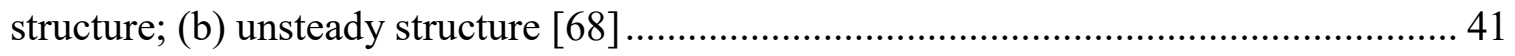

Figure 2-8 Equivalent circuits and corresponding Nyquist plots: (a) purely capacitive coating circuit; (b) simplified Randles circuit; (c) Nyquist plot of purely capacitive coating;

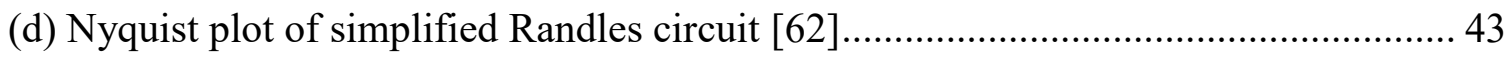

Figure 2-9 Interpretation of the equivalent circuits of a passive metal surface: (a) circuit 1 of $\mathrm{R}(\mathrm{Q}(\mathrm{R}(\mathrm{QR})))$; (b) circuit 2 of $\mathrm{R}(\mathrm{QR})(\mathrm{QR})$; (c) illustration of the model of $\mathrm{R}(\mathrm{Q}(\mathrm{R}(\mathrm{QR})))$;

(d) illustration of the model of $\mathrm{R}(\mathrm{QR})(\mathrm{QR})[69]$ 44 
Figure 3-1 Tescan Vega-II XMU SEM with backscatter electron imaging and an EDX spectroscopy system 49

Figure 3-2 SEM microstructure of Stellite 6 alloy: (a) at low magnification; (b) at high magnification. 50

Figure 3-3 SEM microstructure of 17-4PH stainless steel: (a) at low magnification; (b) at high magnification. 50

Figure 3-4 EDX results of the white phase in Stellite 6 alloy: (a) SEM morphology; (b) EDX spectrum; (c) elemental content table 51

Figure 3-5 EDX results of the black phase in Stellite 6 alloy: (a) SEM morphology; (b) EDX spectrum; (c) elemental content table 51

Figure 3-6 EDX results of the grey phase in Stellite 6 alloy: (a) SEM morphology; (b) EDX spectrum; (c) elemental content table 52

Figure 3-7 EDX results of the martensitic matrix of 17-4PH stainless steel: (a) SEM morphology; (b) EDX spectrum; (c) elemental content table 52

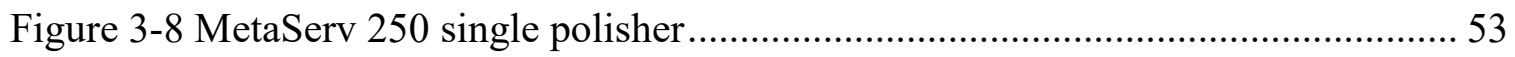

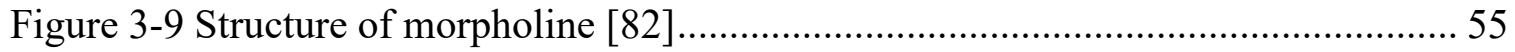

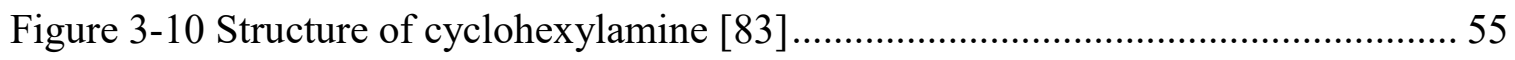

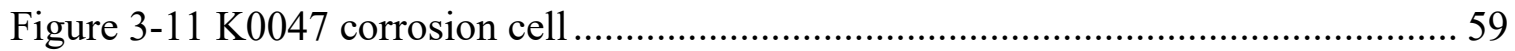

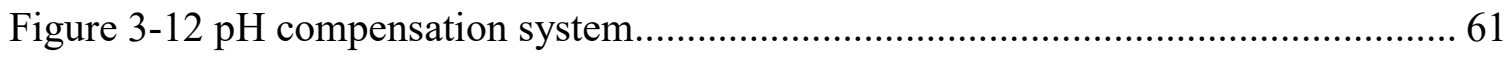

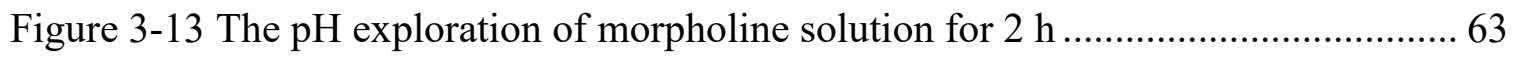

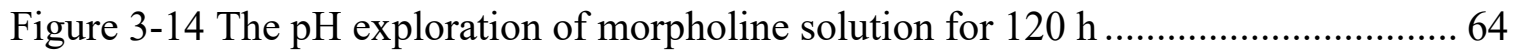

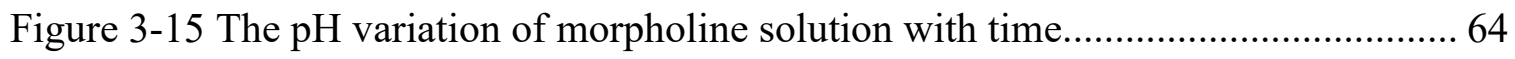

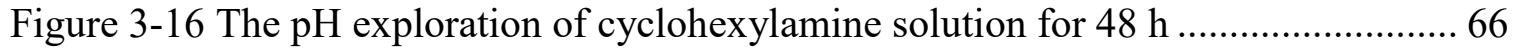


Figure 3-17 System setup: (a) Computer, Solartron 1287 potentiostat, and 1255 FRA; (b) K0047 corrosion cell kit with cable connections; (c) Schematic diagram of the

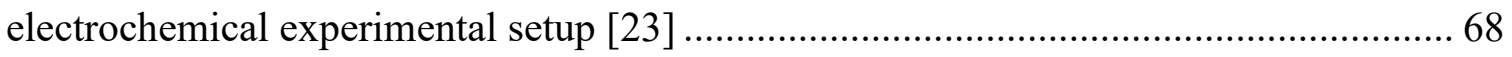

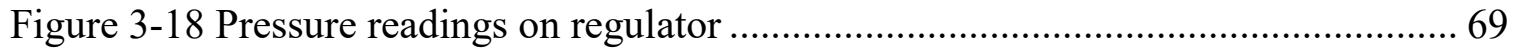

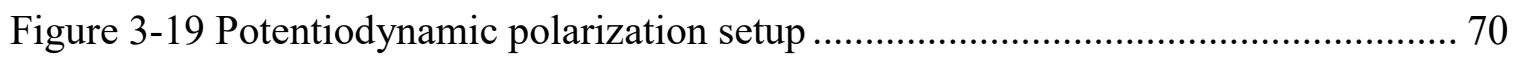

Figure 3-20 System setup of cable connections with RE and CE shorted..................... 72

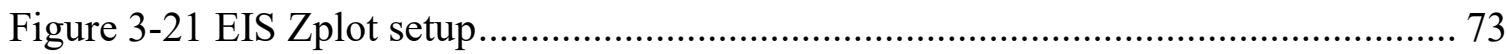

Figure 4-1 Polarization curves of Stellite 6 alloy in morpholine solution ...................... 75

Figure 4-2 Polarization curves of 17-4PH stainless steel in morpholine solution ............ 76

Figure 4-3 Divided phases of polarization curve of Stellite 6 alloy in morpholine solution

Figure 4-4 Divided phases of polarization curve of 17-4PH stainless steel in morpholine

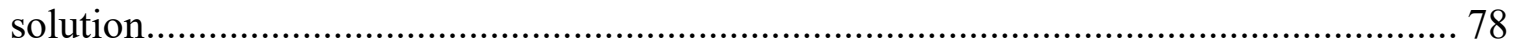

Figure 4-5 Cyclic polarization curve of Stellite 6 alloy in morpholine solution .............. 80

Figure 4-6 Cyclic polarization curve of 17-4PH stainless steel in morpholine solution .. 80 Figure 4-7 Polarization curves of Stellite 6 alloy in cyclohexylamine solution .............. 81 Figure 4-8 Polarization curves of 17-4PH stainless steel in cyclohexylamine solution ... 82 Figure 4-9 Divided phases of polarization curve of Stellite 6 alloy in cyclohexylamine

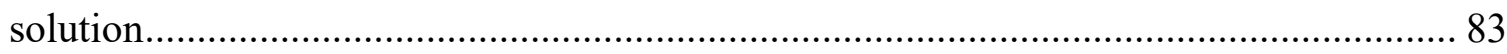

Figure 4-10 Divided phases of polarization curve of 17-4PH stainless steel in

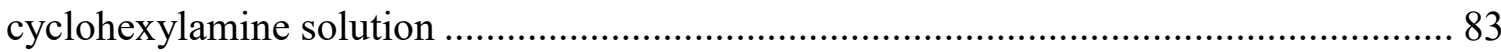

Figure 4-11 Cyclic polarization curve of Stellite 6 alloy in cyclohexylamine solution ... 84 
Figure 4-12 Cyclic polarization curve of 17-4PH stainless steel in cyclohexylamine solution. 85

Figure 4-13 Polarization curves of Stellite 6 alloy in sodium hydroxide solution 86 Figure 4-14 Polarization curves of 17-4PH stainless steel in sodium hydroxide solution 86 Figure 4-15 Divided phases of polarization curve of Stellite 6 alloy in sodium hydroxide solution. 88

Figure 4-16 Divided phases of polarization curve of 17-4PH stainless steel in sodium hydroxide solution 88

Figure 4-17 Cyclic polarization curve of Stellite 6 alloy in sodium hydroxide solution.. 89 Figure 4-18 Cyclic polarization curve of 17-4PH stainless steel in sodium hydroxide solution. 90

Figure 4-19 Equivalent circuit used to fit the EIS data............................................ 91

Figure 4-20 Nyquist plots of Stellite 6 alloy in morpholine solution .......................... 92

Figure 4-21 Nyquist plots of 17-4PH stainless steel in morpholine solution ................. 92

Figure 4-22 Nyquist plots of Stellite 6 alloy in cyclohexylamine solution .................... 94

Figure 4-23 Nyquist plots of 17-4PH stainless steel in cyclohexylamine solution .......... 94

Figure 4-24 Nyquist plots of Stellite 6 alloy in sodium hydroxide solution................... 96

Figure 4-25 Nyquist plots of 17-4PH stainless steel in sodium hydroxide solution......... 96 Figure 5-1 Cyclic polarization curve of Stellite 6 alloy tested in morpholine solution at high potentials 100

Figure 5-2 Photo of the Stellite 6 alloy sample tested in morpholine solution for cyclic polarization at high potentials. 100 
Figure 5-3 Photo of the Stellite 6 alloy sample tested in morpholine solution under potentiostatic polarization: (a) for $25000 \mathrm{~s}$; (b) for $70000 \mathrm{~s}$ 101

Figure 5-4 Photo of the 17-4PH stainless steel sample tested in morpholine solution under potentiostatic polarization: (a) for $1500 \mathrm{~s}$; (b) for $2550 \mathrm{~s}$ 102

Figure 5-5 Potentiostatic polarization failure test setup for Stellite 6 alloy 103 Figure 5-6 Photos of the Stellite 6 alloy sample tested in morpholine solution under potentiostatic polarization: (a) for $0 \mathrm{~s}$; (b) for $5000 \mathrm{~s}$; (c) for $10000 \mathrm{~s}$; (d) for $15000 \mathrm{~s}$; (e) for $20000 \mathrm{~s}$; (f) for $25000 \mathrm{~s}$ 104

Figure 5-7 Variation of current with time of the Stellite 6 alloy sample tested in morpholine solution under potentiostatic polarization 104

Figure 5-8 SEM images of corroded surface of the Stellite 6 alloy sample tested in morpholine solution: (a) at low magnification; (b) at high magnification 106

Figure 5-9 EDX results of the white area on the corroded surface of the Stellite 6 alloy sample tested in morpholine solution: (a) SEM morphology; (b) EDX spectrum; (c) elemental content table 106

Figure 5-10 EDX results of the black area on the corroded surface of the Stellite 6 alloy sample tested in morpholine solution: (a) SEM morphology; (b) EDX spectrum; (c) elemental content table 107 Figure 5-11 EDX results of the grey area on the corroded surface of the Stellite 6 alloy sample tested in morpholine solution: (a) SEM morphology; (b) EDX spectrum; (c) elemental content table 107 
Figure 5-12 Photos of the Stellite 6 alloy sample tested in cyclohexylamine solution under potentiostatic polarization: (a) for $0 \mathrm{~s}$; (b) for $5000 \mathrm{~s}$; (c) for $10000 \mathrm{~s}$; (d) for $15000 \mathrm{~s}$; (e) for $20000 \mathrm{~s}$; (f) for $25000 \mathrm{~s}$ 109

Figure 5-13 Variation of current with time of the Stellite 6 alloy sample tested in cyclohexylamine solution under potentiostatic polarization. 109

Figure 5-14 SEM images of corroded surface of the Stellite 6 alloy sample tested in cyclohexylamine solution: (a) at low magnification; (b) at high magnification

Figure 5-15 EDX results of the white area on the corroded surface of the Stellite 6 alloy sample tested in cyclohexylamine solution: (a) SEM morphology; (b) EDX spectrum; (c) elemental content table

Figure 5-16 EDX results of the black area on the corroded surface of the Stellite 6 alloy sample tested in cyclohexylamine solution: (a) SEM morphology; (b) EDX spectrum; (c) elemental content table 112

Figure 5-17 EDX results of the grey area on the corroded surface of the Stellite 6 alloy sample tested in cyclohexylamine solution: (a) SEM morphology; (b) EDX spectrum; (c) elemental content table 112 Figure 5-18 Photos of the Stellite 6 alloy sample tested in sodium hydroxide solution under potentiostatic polarization: (a) for $0 \mathrm{~s}$; (b) for $5000 \mathrm{~s}$; (c) for $10000 \mathrm{~s}$; (d) for $15000 \mathrm{~s}$; (e) for $20000 \mathrm{~s}$; (f) for $25000 \mathrm{~s}$ 114

Figure 5-19 Variation of current with time of the Stellite 6 alloy sample tested in sodium hydroxide solution under potentiostatic polarization 114 Figure 5-20 SEM images of corroded surface of the Stellite 6 alloy sample tested in sodium hydroxide solution: (a) at low magnification; (b) at high magnification..... 115 
Figure 5-21 EDX results of the white area on the corroded surface of the Stellite 6 alloy sample tested in sodium hydroxide solution: (a) SEM morphology; (b) EDX spectrum; (c)

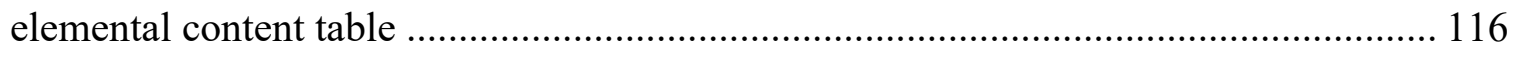
Figure 5-22 EDX results of the black area on the corroded surface of the Stellite 6 alloy sample tested in sodium hydroxide solution: (a) SEM morphology; (b) EDX spectrum; (c) elemental content table 116 Figure 5-23 EDX results of the grey area on the corroded surface of the Stellite 6 alloy sample tested in sodium hydroxide solution: (a) SEM morphology; (b) EDX spectrum; (c) elemental content table 117

Figure 5-24 Potentiostatic polarization failure test setup for 17-4PH stainless steel ..... 118 Figure 5-25 Photos of the 17-4PH stainless steel sample tested in morpholine solution under potentiostatic polarization: (a) for $0 \mathrm{~s}$; (b) for $1500 \mathrm{~s}$ 119 Figure 5-26 Variation of current with time of the 17-4PH stainless steel sample tested in morpholine solution under potentiostatic polarization 119 Figure 5-27 SEM images of corroded surface of the 17-4PH stainless steel sample tested in morpholine solution: (a) at low magnification; (b) at high magnification 121 Figure 5-28 EDX results of the white area in the porous region on the corroded surface of the 17-4PH stainless steel sample tested in morpholine solution: (a) SEM morphology; (b) EDX spectrum; (c) elemental content table 122

Figure 5-29 EDX results of the grey area in the porous region on the corroded surface of the 17-4PH stainless steel sample tested in morpholine solution: (a) SEM morphology; (b) EDX spectrum; (c) elemental content table 122 
Figure 5-30 EDX results of the dark grey area in the smooth region on the corroded surface of the 17-4PH stainless steel sample tested in morpholine solution: (a) SEM morphology; (b) EDX spectrum; (c) elemental content table 123

Figure 5-31 EDX results of the light grey area in the smooth region on the corroded surface of the 17-4PH stainless steel sample tested in morpholine solution: (a) SEM morphology; (b) EDX spectrum; (c) elemental content table 123

Figure 5-32 Photos of the 17-4PH stainless steel sample tested in cyclohexylamine solution under potentiostatic polarization: (a) for $0 \mathrm{~s}$; (b) for $1500 \mathrm{~s}$. 124 Figure 5-33 Variation of current with time of the 17-4PH stainless steel sample tested in cyclohexylamine solution under potentiostatic polarization. 124

Figure 5-34 SEM images of corroded surface of the 17-4PH stainless steel sample tested in cyclohexylamine solution: (a) at low magnification; (b) at high magnification......... 126 Figure 5-35 EDX results of the dark area on the corroded surface of the 17-4PH stainless steel sample tested in cyclohexylamine solution: (a) SEM morphology; (b) EDX spectrum; (c) elemental content table 126

Figure 5-36 EDX results of the light area on the corroded surface of the 17-4PH stainless steel sample tested in cyclohexylamine solution: (a) SEM morphology; (b) EDX spectrum; (c) elemental content table 127 Figure 5-37 Photos of the 17-4PH stainless steel sample tested in sodium hydroxide solution under potentiostatic polarization: (a) for $0 \mathrm{~s}$; (b) for $1500 \mathrm{~s}$ 128 Figure 5-38 Variation of current with time of the 17-4PH stainless steel sample tested in sodium hydroxide solution under potentiostatic polarization 128 
Figure 5-39 SEM images of corroded surface of the 17-4PH stainless steel sample tested in sodium hydroxide solution: (a) at low magnification; (b) at high magnification....... 129 Figure 5-40 EDX results of the dark area on the corroded surface of the 17-4PH stainless steel sample tested in morpholine solution: (a) SEM morphology; (b) EDX spectrum; (c) elemental content table 130

Figure 5-41 EDX results of the light area on the corroded surface of the 17-4PH stainless steel sample tested in morpholine solution: (a) SEM morphology; (b) EDX spectrum; (c) elemental content table 130

Figure 6-1 Variation of current with time for Stellite 6 alloy under potentiostatic polarization. 137

Figure 6-2 Variation of current with time for 17-4PH stainless steel under potentiostatic polarization 138

Figure 6-3 Pourbaix diagram for the chromium-water system [99] 141

Figure 6-4 Pourbaix diagram for the cobalt-water system [101] 141

Figure 6-5 Pourbaix diagram for the iron-water system [103] 142

Figure 6-6 Possible amine adsorption mechanism: (a) chemisorption on the metal surface; (b) hydrogen bonding to the oxidized surface [111]..... 143 


\section{List of Appendices}

Figure A1 The electrical tape for collecting the corrosion products 164

Figure A2 SEM images of the corrosion products of the Stellite 6 alloy sample tested in morpholine solution: (a) region 1; (b) region 2 165

Figure A3 EDX results of region 1 of the corrosion products on the Stellite 6 alloy sample tested in morpholine solution: (a) SEM morphology; (b) EDX spectrum; (c) elemental content table. 165

Figure A4 EDX results of region 2 of the corrosion products on the Stellite 6 alloy sample tested in morpholine solution: (a) SEM morphology; (b) EDX spectrum; (c) elemental content table 166

Figure A5 SEM images of the corrosion products of the Stellite 6 alloy sample tested in cyclohexylamine solution: (a) region 1 ; (b) region 2 . 166

Figure A6 EDX results of region 1 of the corrosion products on the Stellite 6 alloy sample tested in cyclohexylamine solution: (a) SEM morphology; (b) EDX spectrum; (c) elemental content table 167

Figure A7 EDX results of region 2 of the corrosion products on the Stellite 6 alloy sample tested in cyclohexylamine solution: (a) SEM morphology; (b) EDX spectrum; (c) elemental content table 167

Figure A8 SEM images of the corrosion products of the 17-4PH stainless steel sample tested in morpholine solution: (a) region 1; (b) region 2 168 
Figure A9 EDX results of region 1 of the corrosion products on the 17-4PH stainless steel sample tested in morpholine solution: (a) SEM morphology; (b) EDX spectrum; (c) elemental content table

Figure A10 EDX results of region 2 of the corrosion products on the 17-4PH stainless steel sample tested in morpholine solution: (a) SEM morphology; (b) EDX spectrum; (c) elemental content table 169

Figure A11 SEM images of the corrosion products of the 17-4PH stainless steel sample tested in cyclohexylamine solution: (a) region 1 ; (b) region 2 170 Figure A12 EDX results of region 1 of the corrosion products on the 17-4PH stainless steel sample tested in cyclohexylamine solution: (a) SEM morphology; (b) EDX spectrum; (c) elemental content table 170 Figure A13 EDX results of region 2 of the corrosion products on the 17-4PH stainless steel sample tested in cyclohexylamine solution: (a) SEM morphology; (b) EDX spectrum; (c) elemental content table 171 


\section{Acronyms}

\begin{tabular}{|c|c|}
\hline $\mathrm{AC}$ & Alternating Current \\
\hline ASTM & American Society for Testing and Materials \\
\hline $\mathrm{CE}$ & Counter Electrode \\
\hline $\mathrm{CPE}$ & Constant Phase Element \\
\hline $\mathrm{DC}$ & Direct Current \\
\hline DR & Distribution Ratio \\
\hline EDX & Energy Dispersive X-ray \\
\hline EIS & Electrochemical Impedance Spectroscopy \\
\hline FRA & Frequency Response Analyzer \\
\hline HIP & Hot Isostatic Press \\
\hline OCP & Open Circuit Potential \\
\hline PREN & Pitting Resistance Equivalent Number \\
\hline $\mathrm{RE}$ & Reference Electrode \\
\hline SCE & Saturated Calomel Electrode \\
\hline SEM & Scanning Electron Microscopy \\
\hline UNS & Unified Numbering System \\
\hline WE & Working Electrode \\
\hline XPS & X-ray Photoelectron Spectroscopy \\
\hline
\end{tabular}




\section{Chapter 1: Introduction}

\subsection{Research background and significance}

The seat surface of a control valve is critical for reliable metal-to-metal sealing operation. In coal power plants, the seat faces of control valves must be capable of withstanding very high contact stress without cracking, severe wear, corrosion, and cavitation without damage. As a result, the reduction or elimination of surface damage at high impact loads and in severe operating environments has become an important consideration in the valve design. To enhance the seat face of a control valve, a hardfacing layer is usually applied on it. Therefore, the service life of a control valve relies significantly on the extent of seat face damage and the impact toughness of the hard-faced layer. Stellite 6 alloy has been popularly employed as the hardfacing material for the seat faces of various control valves due to its unique chemical composition of $\mathrm{Co} 29 \mathrm{Cr} 4.5 \mathrm{~W} 1.5 \mathrm{Mo} 1.2 \mathrm{C}$ (in weight) and excellent combined mechanical, corrosion, wear, and high temperature properties [1-3]. It is frequently deposited with welding processes and laser cladding methods.

However, in January 1991, Bush published an article titled "Be alert to new valve threat: erosion/corrosion in feed water" in an edition of Power Magazine. This article discussed that "Loss of corrosion resistance in Alloy 6 comes to notice most often in boiler feed water service where the water treatment includes hydrazine or other amine derivative." [4]. Moreover, it analyzed a failed Alloy 6 valve seat, as shown in Figure 1-1 and Figure 1-2, concluding with a suggestion to avoid using cobalt-containing alloys in feed water service unless the feed water is known to be compatible with Alloy 6. However, this problem has 
not been reported in other publications, and the industry experience of Kennametal Stellite Inc. leads them to believe that this type of failure is rare.

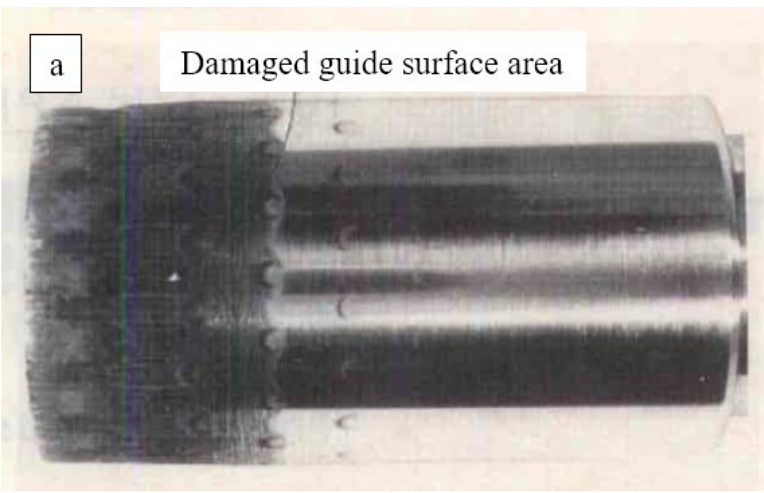

a. Erosion/corrosion damage of valve plug occurred at seat and well along the plug

b. Seat end of plug had damage band next to outer diameter. Cut was for specimen removal

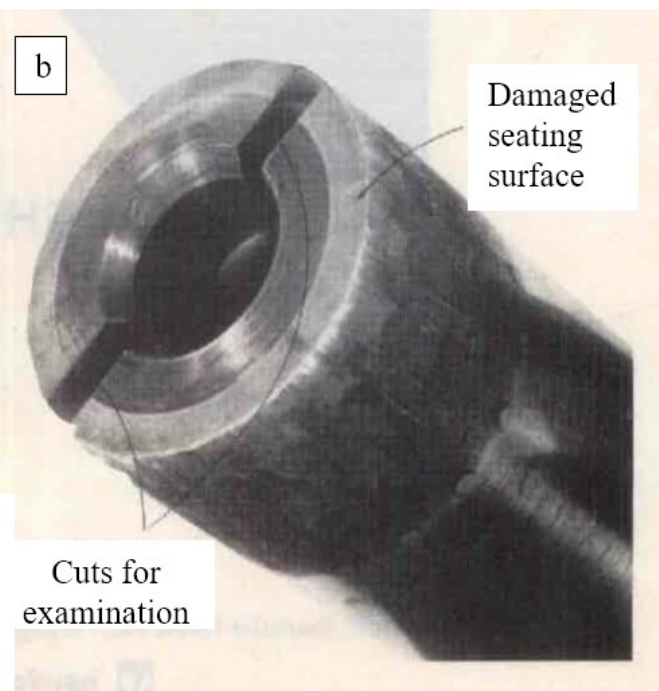

Figure 1-1 Erosion/corrosion damage of the valve seat [4]

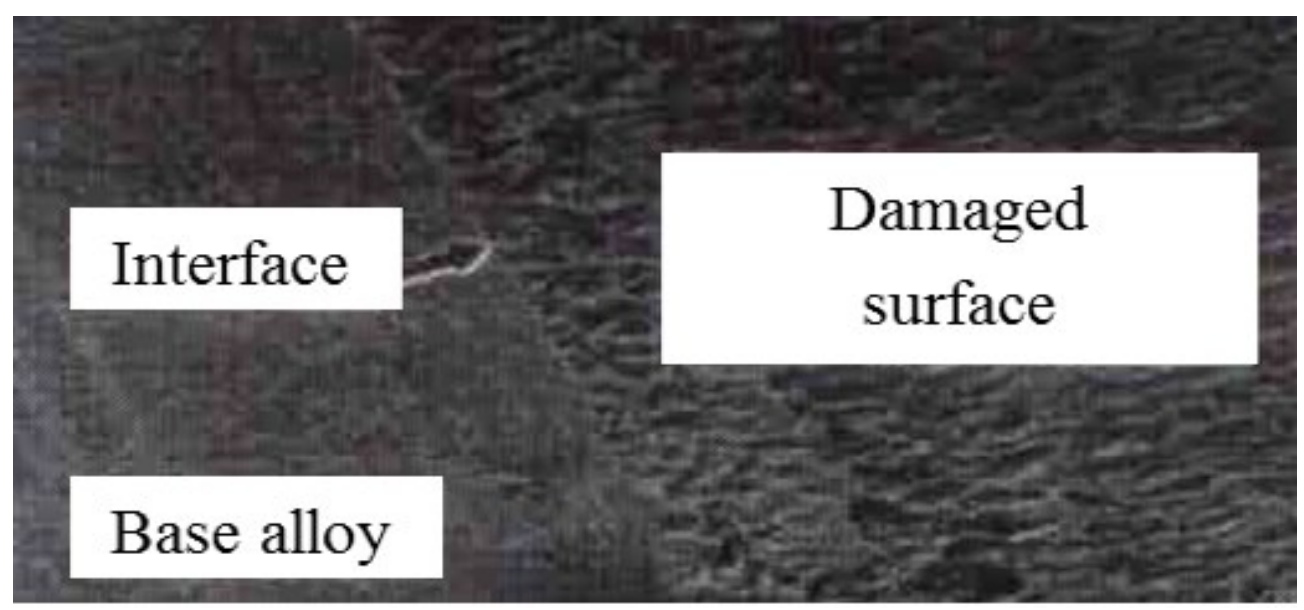

Figure 1-2 Interface of Stellite 6 alloy showing cratered face [4]

Although the cratered surface resulted from the attack of erosion/corrosion, which can be schematically depicted in Figure 1-3 [5], the real erosion/corrosion attack cannot be easily simulated and tested simultaneously using electrochemical methods. Thus, the goal of investigating the relatively complicated erosion/corrosion behavior must be simplified to 
master the corrosion behavior of the tested material in the corrosive media in the lab environment. The effect of erosion/corrosion can be considered as a severely exaggerated result of the corrosion process.

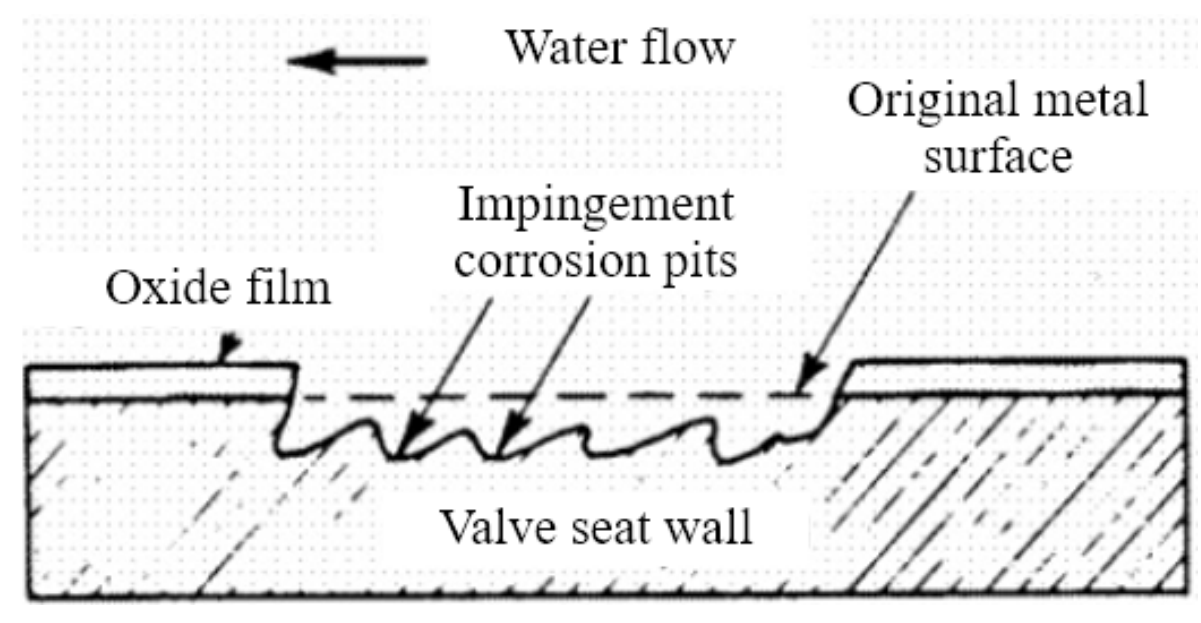

Figure 1-3 Schematic illustration of erosion/corrosion attack [5]

The basic concept of corrosion is a deterioration process due to the chemical or electrochemical interactions between substances such as metals, polymers, ceramics, and their surroundings, but it mostly refers to the corrosion process of metals [6], which leads to material loss or dissolving of the constituents of the environment. Although corrosion condition differs from metal to metal, four factors govern the corrosion system: the chemical composition and microstructure of the metal, the chemical composition of the environment, the physical parameters such as temperature, convection, irradiation and the mechanical forces including tensile stresses, shocks or friction [7].

Although possible mechanisms of the corrosion loss for Stellite 6 alloy in amine environments have been reported in previous research [4], the performance of this alloy in 
such a service condition has not been well understood. For example, it has been theorized (but not proven) by Bush that the presence of the amine compounds somehow prevents the reformation of the passive oxide layer on Stellite 6 alloy after it has been removed by high-velocity flow impingement. What is not understood is why this only occurs on cobalt alloys and not on other materials, for example, austenitic stainless steels, which also rely on a passive oxide layer for corrosion protection. This unsolved problem has become urgent and has restricted significantly the application of Stellite alloys for boiler feed water service in power plants as well as in refineries where amine compounds are used to absorb unwanted dissolved gas — primarily hydrogen sulfide from liquid hydrocarbons, and in gas treatment plants where amine compounds are used to absorb hydrogen sulfide and carbon dioxide. To devise a solution for the problem, therefore, this research was aimed to investigate the reaction mechanisms of Stellite alloys, such as Stellite 6 alloy in amine media. 17-4PH stainless steel is also studied for comparison because this material is reported to be selected as a typical hardened stainless steel material showing the least attack in most cases.

There are two types of amines, filming amine and neutralizing amine. The former is insoluble with water at room temperature so that only the latter can be tested in the lab. Neutralizing amines, such as morpholine, cyclohexylamine and diethylaminoethanol are commonly present in feed water system. Since the feedwater of power generation plants cannot be obtained for this research, to simply simulate the industrial amine environment, morpholine solution with $\mathrm{pH} 9.5$ was selected to be the corrosive medium for the corrosion studies of Stellite 6 alloy and 17-4PH stainless steel in the first try, because it is a typical 
amine medium. To further this research, Stellite 6 alloy and 17-4PH stainless steel were also tested in cyclohexylamine solution with $\mathrm{pH} 9.5$ to examine if different amine media with the same $\mathrm{pH}$ can result in any change in the corrosion behavior of the materials. On the other hand, the neutralizing amines are known for the hydrolysis of amines to form hydroxyl $\left(\mathrm{OH}^{-}\right)$ions, which boost the $\mathrm{pH}$ of amine solutions [8]. In order to take into account this factor in the corrosion study, sodium hydroxide was chosen as another corrosive medium to offset the effect of $\mathrm{pH}$ change, because sodium hydroxide solution could provide the same amount of hydroxyl ions without introducing other unwanted variables into the testing environment.

\subsection{Research tasks and outcomes}

\subsubsection{Research tasks}

Aiming at solving the industry problems, this research has completed the following tasks:

(1) Simplify the industrial problems into the tasks that could be carried out in lab conditions.

(2) Identify the microstructures of Stellite 6 alloy and 17-4PH stainless steel using SEM with EDX spectrum.

(3) Create a relatively constant $\mathrm{pH}$ system for morpholine, cyclohexylamine and sodium hydroxide solutions.

(4) Conduct EIS, potentiodynamic polarization and cyclic polarization tests on Stellite 6 alloy and $17-4 \mathrm{PH}$ stainless steel in morpholine, cyclohexylamine and sodium hydroxide solutions with $\mathrm{pH} 9.5$. 
(5) Analyze the data from EIS and polarization tests.

(6) Make comparisons of the experimental results between Stellite 6 alloy and 17-4PH stainless steel in morpholine, cyclohexylamine and sodium hydroxide solutions, respectively.

(7) Provide suggestions on which material has better corrosion resistance in the amine environments.

(8) Perform failure tests on Stellite 6 alloy and 17-4PH stainless steel in morpholine, cyclohexylamine and sodium hydroxide solutions and investigate the failure mechanisms with the analyses of corroded surfaces using SEM.

\subsubsection{Research outcomes}

With the completion of these tasks, the following outcomes have been derived:

(1) The microstructures and phase identification of Stellite 6 alloy and 17-4PH stainless steel were obtained.

(2) The $\mathrm{pH}$ compensation systems were created for morpholine and cyclohexylamine solutions to make the $\mathrm{pH}$ level stable.

(3) The $\mathrm{pH}$ value of sodium hydroxide solution can be maintained at a relatively steady level by purging argon gas through the corrosion cell.

(4) The EIS curves of Stellite 6 alloy and 17-4PH stainless steel were obtained in morpholine, cyclohexylamine and sodium hydroxide solutions.

(5) Cyclic potentiodynamic polarization curves of Stellite 6 alloy and 17-4PH stainless steel were obtained in morpholine, cyclohexylamine and sodium hydroxide solutions. 
(6) Comparison results of experimental data between Stellite 6 alloy and 17-4PH stainless steel in morpholine, cyclohexylamine, and sodium hydroxide solutions were obtained.

(7) Comparison results of experimental data of Stellite 6 alloy in morpholine, cyclohexylamine and sodium hydroxide solutions were obtained.

(8) Comparison results of experimental data of $17-4 \mathrm{PH}$ stainless steel in morpholine, cyclohexylamine and sodium hydroxide solutions were obtained.

(9) The better candidate between the tested materials for amine environment applications was suggested.

(10) Failure occurred on Stellite 6 alloy and 17-4PH stainless steel when conducting the potentiostatic test on them in morpholine, cyclohexylamine and sodium hydroxide solutions at a high potential $(4 \mathrm{~V})$ over a certain period of time. The microstructural changes of the failed samples were analyzed using SEM/EDX, and the obtained results explained the failure mechanisms of the materials.

\subsection{Organization of this thesis}

This thesis is a summary of the present research. In total seven chapters cover the entire contents of the research, including introduction, literature review, experimental details, experimental results and analyses, discussion on results, conclusions, and future work. Furthermore, the contents of each chapter are summarized below:

Chapter 1 is an introduction to this thesis. The background, significance, objectives, tasks, and outcomes of this research are described. The organization of the thesis is outlined at the end of this chapter. 
Chapter 2 is the literature review. The boiling water systems in power plants are reviewed step by step, from the basic structures of the boiler feed water systems in common power generation plants to the chemical treatments of the boiling feed water, as well as the concerns on the amine environment due to the chemical treatments. The focus of the review is on the materials used for the feed water valves which operate in amine environments, starting from common materials used for the valves, including Stellite alloys and stainless steels. Following that, the review is focused on the previous researches on Stellite 6 alloy and $17-4 \mathrm{PH}$ stainless steel, which are the most popular materials for such applications. Various corrosion study methods are also reviewed in this chapter, with the emphasis on electrochemical methods, which are employed in this research.

Chapter 3 describes the details of the corrosion experiments. It starts with the preparation of the Stellite 6 alloy and 17-4PH stainless steel specimens and the amine solutions. The similarities and differences of the tested solutions are explained. The methods used to control the $\mathrm{pH}$ level in the electrochemical test systems are described, and the testing system setup is presented. The procedures for potentiodynamic polarization, cyclic polarization, and EIS methods are stated in detail.

Chapter 4 presents the experimental results of the potentiodynamic polarization, cyclic polarization, and EIS. Additionally, the results of these three methods for each tested material are discussed. 
Chapter 5 presents the experimental procedures and results of the failure tests using potentiostatic polarization. Additionally, the SEM and EDX results of the polarized sample of each tested material in each solution are presented and discussed.

Chapter 6 discusses the experimental results of potentiodynamic polarization, cyclic polarization, and EIS as well as the results of the failure tests. SEM/EDX analyses on the corroded specimens, Pourbaix diagrams of chromium, cobalt and iron, and the differences of the corrosive media are integrated in the exploration of the corrosion mechanisms of the materials.

Chapter 7 summarizes the outcomes, draws the conclusions, and highlights the significant contributions, of this research. The future work towards the goal of the entire project "Investigation of Corrosion Resistance of Stellite Alloys in Amine Environments" is proposed. 


\section{Chapter 2: Literature Review}

\subsection{Boiling water systems in power generation plants}

\subsubsection{Boiler feed water systems}

It is known that more than sixty percent of the world's power is generated by the combustion of coal, oil and natural gas in thermal power plants [9]. The hot gasses generated by the combustion of the fuels transfer its heat to the feed water in the boiler tubes, which are typically in a large array $[10,11]$. Once the water is heated by the hot gasses, the steam is generated for further use of driving turbine [9]. The steam generated in this process is collected from the boiler tubes in a steam drum before it is sent to the turbine. Figure 2-1 illustrates the typical system layout of a large thermal power generation plant [11]. Once the turbine is coupled to the alternator, the rotation of the turbine blades driven by the steam will generate electrical energy.

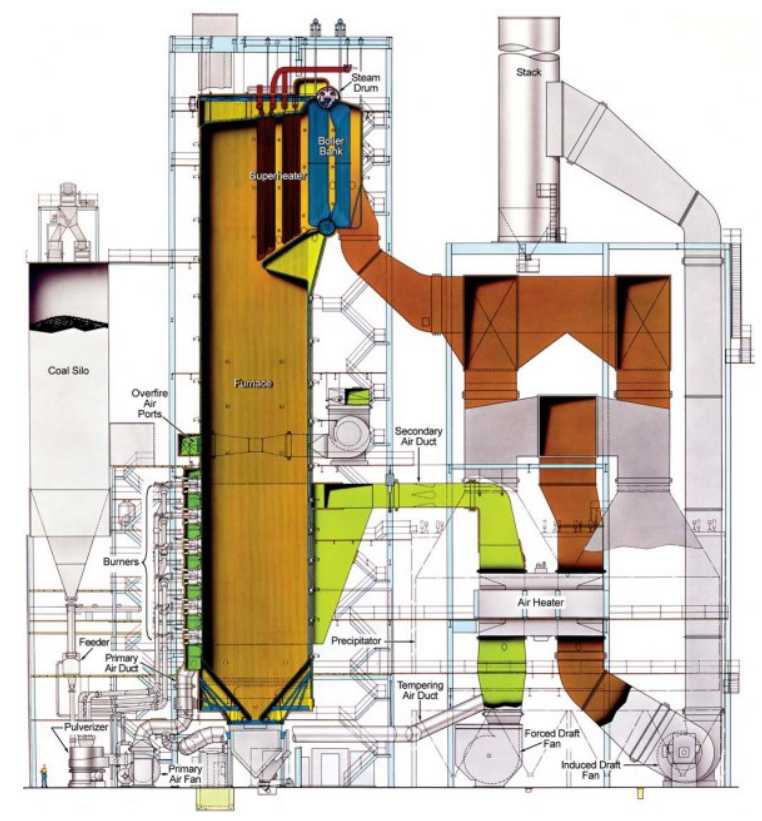

Figure 2-1 Typical thermal power plant system [11] 
The boiler tube is a component that contains feed water of the boiler. The system for the boiler feed water used in the steam boiler is designed for the condensate water to exchange heat with the burning gas which drives the steam turbine. Therefore, the feed water plays an important role in thermal power plants. Figure 2-2 shows the water system used in thermal power plants [12].

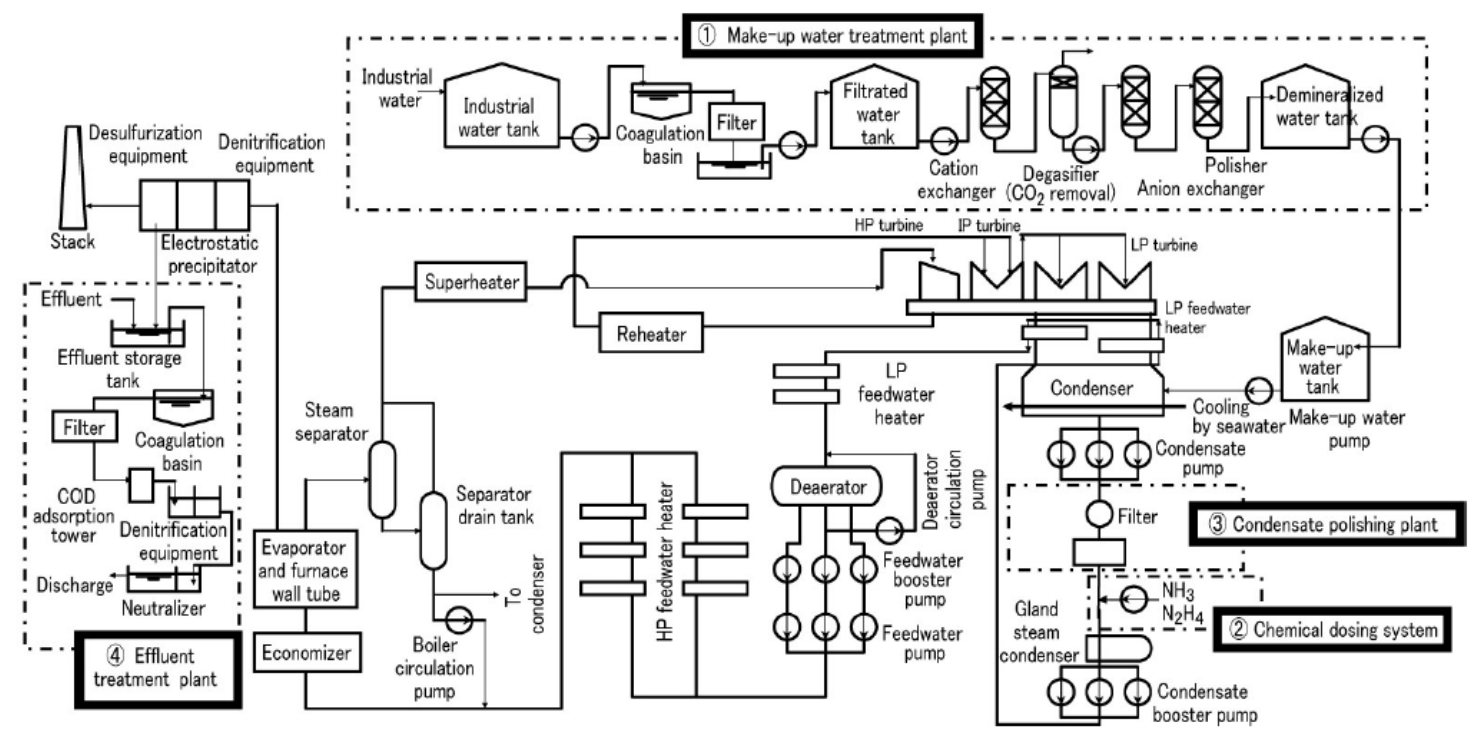

Figure 2-2 Water systems of thermal power plants [12]

The typical water system generally consists of four parts. The first stage of water is condensated water. When it flows towards the boiler, it can be named as boiler feed water. As it flows into the boiler, it becomes the boiler water. After heat exchanging with the hot gas, it becomes steam in the turbine. Finally, it goes back to condensate water again. The makeup water treatment plant, chemical dosing system, condensate polishing plant and effluent treatment plant are also water-related systems in thermal power plants. The makeup water treatment plant is a place where the industrial water can be purified. Chemical dosing system monitors the quality of water and adjusts the quality of water to 
prevent corrosion. Condensate polishing plant is the place where the circulating water can be purified for reuse. To minimize the influence of the waste product produced in thermal power plants, the effluent treatment plant was created to purify the wastewater [12].

\subsubsection{Chemical treatments}

Proper chemical treatment and conditioning of water are required because water is the foundation of all the other components in steam-based power plants. Scaling and corrosion may occur in the boiler tubes, which will result in economic loss if the water treatment is not decent. As the production capacity decreases and the operating cost increases, the economic loss will be enormous [13].

There are commonly three types of impurities in water, suspended solids, dissolved solids, and dissolved gasses, but the percentages of each in water vary from case to case [14]. The suspended solids are the main reason for the corrosion and deposition problems in the boilers; filtration and clarification are two common ways to treat the suspended solids. The filter is always composed of different sizes of gravel, sand, and anthracite coal, but they are good enough to make the concentration of suspended solids to an acceptable level. Similarly, the clarifier is used for the settling process of particulates. This process can be accelerated when coagulant and flocculent are added into the still water in the clarifier. Demineralization, reverse osmosis and ion exchange softening are the methods to treat the dissolved solids because calcium and magnesium can result in scales on the boiler related tubes. These scales are formed when the hardness of water is high. Hence softeners can be added to solve this problem primarily. However, if a higher control in dissolved solids is 
needed, demineralization methods can be used. Demineralizers contain cation beds and anion beds, where cations can exchange hydrogen ions with positive ions (sodium, calcium, magnesium, and iron) and anions can exchange hydroxide ions with negative ions (chloride, sulfate, carbonate, and silica). Reverse osmosis can result in the same result with the demineralization process, but they function in a totally different way. When applying pressure on a certain amount of water through the reverse osmosis membrane, the pure water can go through the membrane. However, the contaminants cannot go through it. Dissolved gasses are always treated via de-aeration, degasification, and de-alkalization pretreatment methods [14]. The deaerators could change physical properties of the temperature and pressure so that the dissolved carbon dioxide and oxygen can be driven off, especially when the water gets warmer. Degasification refers to the removal of the dissolved gasses from the liquid; heating and using membrane contactors [15] are two typical methods in boiler feed water applications. As for de-alkalization, dealkalizers contain ion exchange resins (mostly chloride and some hydroxide) that could be used to exchange chloride for carbonate, bicarbonate, and sulfate.

Therefore, chemicals are added to the water for purification. In addition, this is more necessary in boiler feed water systems for protecting the boiler against corrosion and scale formation and maintaining the steam purity. To treat the water, many chemicals can be added to control the water impurities and the overall chemistry of water, such as alkalinity, chlorides, sulfite, phosphates, dispersants, amines. Figure 2-3 gives a good illustration of how the chemical additives work in the boiler water treatment system [16]. The associated 
problems caused by the impurities in water and common treatment methods are summarized in Table 2-1.

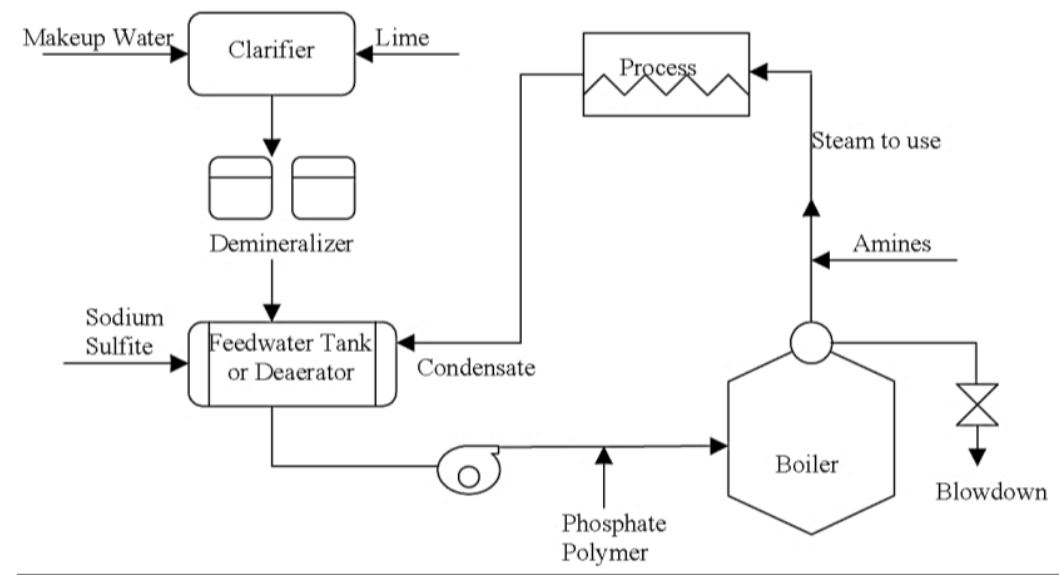

Figure 2-3 Schematic of basic boiler system [16]

Table 2-1 Problems caused by impurities in water [16]

\begin{tabular}{|c|c|c|}
\hline $\begin{array}{c}\text { Impurity (chemical } \\
\text { formula) }\end{array}$ & Problem & $\begin{array}{l}\text { Common chemical treatment } \\
\text { method }\end{array}$ \\
\hline $\begin{array}{l}\text { Alkalinity }\left(\mathrm{HCO}_{3}^{-} \text {, }\right. \\
\left.\mathrm{CO}_{3}{ }^{2-} \text { and } \mathrm{CaCO}_{3}\right)\end{array}$ & $\begin{array}{l}\text { Carryover of feed water into } \\
\text { steam, produce } \mathrm{CO}_{2} \text { in steam } \\
\text { leading to formation of } \\
\text { carbonic acid (acid attack) }\end{array}$ & $\begin{array}{l}\text { Neutralizing amines, filming } \\
\text { amines, combination of both, and } \\
\text { lime-soda. }\end{array}$ \\
\hline $\begin{array}{l}\text { Hardness (calcium } \\
\text { and magnesium } \\
\text { salts, } \mathrm{CaCO} \text { ) } \\
\end{array}$ & $\begin{array}{c}\text { Primary source of scale in heat } \\
\text { exchange equipment }\end{array}$ & $\begin{array}{l}\text { Lime softening, phosphate, } \\
\text { chelates and polymers }\end{array}$ \\
\hline Iron $\left(\mathrm{Fe}^{3+}\right.$ and $\left.\mathrm{Fe}^{2+}\right)$ & $\begin{array}{c}\text { Causes boiler and water line } \\
\text { deposits }\end{array}$ & Phosphate, chelates and polymers \\
\hline Oxygen $\left(\mathrm{O}_{2}\right)$ & $\begin{array}{c}\text { Corrosion of water lines, } \\
\text { boiler, return lines, heat } \\
\text { exchanger equipment, etc. } \\
\text { (oxygen attack) }\end{array}$ & $\begin{array}{l}\text { Oxygen scavengers, filming } \\
\text { amines and deaeration }\end{array}$ \\
\hline pH & $\begin{array}{l}\text { corrosion occurs when } \mathrm{pH} \\
\text { drops below } 8.5\end{array}$ & $\begin{array}{c}\mathrm{pH} \text { can be lowered by addition of } \\
\text { acids and increased by addition of } \\
\text { alkalies }\end{array}$ \\
\hline $\begin{array}{l}\text { Hydrogen sulfide } \\
\left(\mathrm{H}_{2} \mathrm{~S}\right)\end{array}$ & Corrosion & Chlorination \\
\hline Silica $\left(\mathrm{SiO}_{2}\right)$ & $\begin{array}{l}\text { Scale in boilers and cooling } \\
\text { water systems }\end{array}$ & Lime softening \\
\hline
\end{tabular}


In Table 2-2, a variety of guided parameters are suggested by ASME (American Society of Mechanical Engineers) for controlling the good chemistry of water [17]. In all cases, the mechanisms are related to the mechanical damage of the protective film. Environmental factors that control erosion/corrosion include water velocity, oxygen content, $\mathrm{pH}$, temperature, flow geometry, and the presence of particulates in the water. Both mechanical properties of a metal and the nature of the passive film on the metal affect the erosion-corrosion susceptibility [18]. Table 2-3 gives a suggested parameters setting for boiler feed water treatments [19].

Table 2-2 ASME suggested water chemistry limits for industrial watertube, high duty, primary fuel-fired - feed water [17]

\begin{tabular}{|c|c|c|c|c|c|c|c|c|}
\hline $\begin{array}{c}\text { Drum operating } \\
\text { pressure psig (MPa) }\end{array}$ & $\begin{array}{c}0-300 \\
(0-2.07)\end{array}$ & $\begin{array}{c}301-450 \\
(2.08- \\
3.10)\end{array}$ & $\begin{array}{c}451-600 \\
(3.11- \\
4.14)\end{array}$ & $\begin{array}{c}601-750 \\
(4.14- \\
5.17)\end{array}$ & $\begin{array}{c}751-900 \\
(5.18- \\
6.21)\end{array}$ & $\begin{array}{c}901- \\
1000 \\
(6.22- \\
6.89)\end{array}$ & $\begin{array}{c}1001- \\
1500 \\
(6.90- \\
10.34)\end{array}$ & $\begin{array}{c}1501- \\
2000 \\
(10.35- \\
13.79)\end{array}$ \\
\hline $\begin{array}{c}\text { Dissolved oxygen } \\
\text { ppm (mg/L) } \mathrm{O}_{2}- \\
\text { measured before } \\
\text { chemical scavenger } \\
\text { addition }\end{array}$ & $<0.007$ & $<0.007$ & $<0.007$ & $<0.007$ & $<0.007$ & $<0.007$ & $<0.007$ & $<0.007$ \\
\hline $\begin{array}{c}\text { Total iron ppm } \\
(\mathrm{mg} / \mathrm{L}) \mathrm{Fe}\end{array}$ & $\leq 0.1$ & $\leq 0.05$ & $\leq 0.03$ & $\leq 0.025$ & $\leq 0.02$ & $\leq 0.02$ & $\leq 0.01$ & $\leq 0.01$ \\
\hline $\begin{array}{c}\text { Total copper ppm } \\
(\mathrm{mg} / \mathrm{L}) \mathrm{Cu}\end{array}$ & $\leq 0.05$ & $\leq 0.025$ & $\leq 0.02$ & $\leq 0.02$ & $\leq 0.015$ & $\leq 0.01$ & $\leq 0.01$ & $\leq 0.0$ \\
\hline $\begin{array}{l}\text { Total hardness ppm } \\
(\mathrm{mg} / \mathrm{L}) \text { as } \mathrm{CaCO}_{3}\end{array}$ & $\leq 0.3$ & $\leq 0.3$ & $\leq 0.2$ & $\leq 0.2$ & $\leq 0.1$ & $\leq 0.05$ & ND & ND \\
\hline pH $@ 25^{\circ} \mathrm{C}$ & $8.3-10.0$ & $8.3-10.0$ & $8.3-10.0$ & $8.3-10.0$ & $8.3-10.0$ & $8.8-9.6$ & $8.8-9.6$ & $8.8-9.6$ \\
\hline $\begin{array}{c}\text { Chemicals for } \\
\text { preboiler system } \\
\text { protection }\end{array}$ & NS & NS & NS & NS & NS & NS & NS & NS \\
\hline $\begin{array}{c}\text { Nonvolatile TOC } \\
\text { ppm (mg/L) C }\end{array}$ & $<1$ & $<1$ & $<0.5$ & $<0.5$ & $<0.5$ & $<0.2$ & $<0.2$ & $<0.2$ \\
\hline $\begin{array}{c}\text { Oily matter ppm } \\
(\mathrm{mg} / \mathrm{L})\end{array}$ & $<1$ & $<1$ & $<0.5$ & $<0.5$ & $<0.5$ & $<0.2$ & $<0.2$ & $<0.2$ \\
\hline
\end{tabular}


Table 2-3 Boiler feed water treatments for once-through boilers [19]

\begin{tabular}{|c|c|}
\hline Boiler type & Once-through boiler \\
\hline Water treatment type & All volatile treatment \\
\hline pH (at $\left.25^{\mathbf{0}} \mathbf{C}\right)$ & $9.0-9.7$ \\
\hline Cation conductivity $(\mathbf{m S} / \mathbf{m})$ & $\leqq 0.025$ \\
\hline Dissolved oxygen $(\boldsymbol{\mu g} / \mathbf{L})$ & $\leqq 7$ \\
\hline Chemicals & Ammonia hydrazine \\
\hline
\end{tabular}

\subsubsection{Concerns on amine environments}

Water treatment programs are complex and vary from plant to plant. Many factors may result in the failure of the valve seats. Simplifications may be needed to make the variables controllable so that the mechanisms of the failure can be studied. As Bush [4] noticed that the failure might result from the impact of amines so that the investigation in whether amines do have effects on the valve materials is meaningful. However, amines also have different functions. Neutralizing amines and the filming amines are being used with good results [20]. The main reason for using the filming amines is to coat the inside of the return condensate system further protecting it from corrosion. Neutralizing amines are volatile alkaline materials used for adjusting the $\mathrm{pH}$ of the feed water and play an effective role in combating with carbon dioxide corrosion, but they are useless against the oxygen corrosion $[14,20]$. The most common filming amines $[16,21]$ are octadecylamine and ethoxylated soya amine, but both of these filming amines are insoluble in water at room temperature, unless they have been heated to a very high temperature, because they are very high molecular weight amines with long hydrocarbon chains. Therefore, the experiments focused on filming amines cannot be done in a lab environment. 
As a simplification, morpholine, which is a common neutralizing amine, was chosen to start with in the thesis. The $\mathrm{pH}$ was set to be around 9.5 at room temperature as a compromising result of the $\mathrm{pH}$ ranges defined in Table 2-2 and Table 2-3, because the measurement of $\mathrm{pH}$ for a certain concentration of the solution may change and result in a range when accounting for the experimental and instrumental errors. The tests in cyclohexylamine and sodium hydroxide solutions at the same $\mathrm{pH}$ level were performed for the comparison purposes.

\subsection{Materials used for feed water valves}

\subsubsection{Common materials used for feed water valves}

Common materials used for feed water valves are given in Table 2-4. These alloys can be classified into martensitic, precipitation hardening, duplex, austenitic and super austenitic alloys. The semi-quantitative method for estimating the relative corrosion resistance of materials has been developed for acid chloride environments based on the compositions of the materials. PREN is an abbreviation of the Pitting Resistance Equivalent Number. Base on this method, the data in Table 2-4 were derived from the alloys' critical pitting temperatures in ferric chloride. The calculated PREN numbers allow engineers to have a primary understanding of the corrosion resistance of the materials in acid environments. 
Table 2-4 Typical chemical compositions (in weight), mechanical properties, and PREN of cast stainless steels used for pumps and valves [21]

\begin{tabular}{|c|c|c|c|c|c|c|c|c|c|c|}
\hline Cast grade & $\mathrm{C}_{\max }$ & $\mathrm{Cr}$ & $\mathbf{N i}$ & Mo & $\mathbf{N}$ & \multicolumn{2}{|c|}{$\begin{array}{c}\text { Yield } \\
\text { strength } \\
\text { MPa (ksi) }\end{array}$} & \multicolumn{2}{|c|}{$\begin{array}{c}\text { Tensile } \\
\text { strength MPa } \\
\text { (ksi) }\end{array}$} & $\operatorname{PREN}^{(5)}$ \\
\hline \multicolumn{11}{|c|}{ White cast irons } \\
\hline $28 \% \mathrm{Cr}$ & $\begin{array}{l}2.0- \\
3.3\end{array}$ & $\begin{array}{c}23- \\
30\end{array}$ & - & $\begin{array}{c}3.0 \\
\max \end{array}$ & - & & - & \multicolumn{2}{|c|}{$\begin{array}{l}\text { Hardened to } \\
699 \mathrm{Bhn}, \\
59 \mathrm{HRC}\end{array}$} & \\
\hline \multicolumn{11}{|l|}{ Martensitic } \\
\hline CA-15 & 0.15 & 12.8 & 1 & 0.5 & - & 450 & -65 & 620 & -90 & 13 \\
\hline CA-6NM & 0.06 & 12.8 & 4 & 0.7 & - & 515 & -80 & \multicolumn{2}{|c|}{$760(110)$} & 14 \\
\hline \multicolumn{11}{|l|}{$\begin{array}{c}\text { CB-6 } \\
\text { 16Cr5Ni1Mo }\end{array}$} \\
\hline DIN $1.4405^{(1)}$ & 0.03 & 16 & 5 & 1 & - & 540 & -78 & \multicolumn{2}{|c|}{$900(130)$} & 18 \\
\hline \multicolumn{11}{|c|}{ Precipitation hardening } \\
\hline CB-7Cu-1 & 0.07 & 16.6 & 4.1 & - & 0.05 & \multicolumn{2}{|c|}{$670(97)^{(2)}$} & \multicolumn{2}{|c|}{$860(125)^{(2)}$} & 17 \\
\hline $\mathrm{CB}-7 \mathrm{Cu}-2$ & 0.07 & 15.3 & 5 & - & 0.05 & \multicolumn{2}{|c|}{$670(97)^{(2)}$} & \multicolumn{2}{|c|}{$860(125)^{(2)}$} & 16 \\
\hline \multicolumn{11}{|l|}{ Duplex } \\
\hline $\begin{array}{c}\text { CD- } \\
\text { 61VIN(3A) }\end{array}$ & 0.06 & 25.5 & 5 & 2.1 & 0.2 & 450 & -65 & 655 & -95 & 35 \\
\hline $\begin{array}{c}\text { CD-4MCuN } \\
\text { (1B) }\end{array}$ & 0.04 & 25.5 & 5.4 & 2 & 0.2 & 485 & -70 & \multicolumn{2}{|c|}{$690(100)$} & 30 \\
\hline J92205 & 0.03 & 22.5 & 5.5 & 3 & 0.2 & 415 & -60 & 620 & -90 & 34 \\
\hline \multicolumn{11}{|l|}{ Austenitic } \\
\hline CF-3 & 0.03 & 19 & 10 & & - & 205 & -30 & 485 & -70 & 19 \\
\hline CF-8 & 0.08 & 19.5 & 9.5 & - & - & 205 & -30 & 485 & -70 & 19 \\
\hline CF-3M & 0.03 & 19 & 11 & 2.5 & - & 205 & -30 & 485 & -70 & 25 \\
\hline CF-8M & 0.08 & 19.5 & 10.5 & 2.5 & - & 205 & -30 & 485 & -70 & 25 \\
\hline CG-3M & 0.03 & 19.5 & 11 & 3.5 & - & 240 & -35 & 515 & -75 & 29 \\
\hline CG-8M & 0.08 & 19.5 & 11 & 3.5 & - & 240 & -35 & 520 & -75 & 29 \\
\hline CN-7M & 0.07 & 20.5 & 29 & 2.5 & - & 170 & -25 & 425 & -62 & 27 \\
\hline CU-5MCUC ${ }^{(3)}$ & 0.05 & 21 & 41 & 3 & - & 240 & -35 & 520 & -75 & 30 \\
\hline \multicolumn{11}{|c|}{ Superaustenitic } \\
\hline CK-3MCuN & 0.025 & 20 & 18.5 & 6.5 & 0.2 & 260 & -38 & 550 & -80 & 38 \\
\hline CN-3MN & 0.03 & 21 & 24.5 & 6.5 & 0.2 & 260 & -38 & 550 & -80 & 41 \\
\hline $654 S M 0^{(4)}$ & 0.01 & 24.5 & 22 & 7.5 & 0.5 & 440 & -64 & \multicolumn{2}{|c|}{$725(106)$} & 57 \\
\hline \multicolumn{11}{|c|}{$\begin{array}{l}\text { (1) German alloy designation. } \\
\text { (2) Minimum; can be increased by variations in heat treatment } \\
\text { (3) Contains } 1.0 \mathrm{Nb} \\
\text { (4) Designation not yet assigned } \\
\text { (5) The PREN rankings, while useful in bleach plant acidic chloride environments, may } \\
\text { not be directly applicable to all environments. }\end{array}$} \\
\hline
\end{tabular}


In steam-powered plants, the possibility that the steam contains abrasive materials is very high. If an alloy has high tensile strength, it may also exhibit high hardness and good working hardening characteristics, that is, the good erosion/abrasion resistance of a material is associated with high hardness proportionally. Table $2-5$ shows the relative erosion/abrasion resistance of various alloys as a basic guide. There are six groups of alloys in Table 2-5. The materials in group 1 show excellent erosion/abrasion resistance which allows them to serve in most severe circumstances [21]. Stellite 6 Co-base alloy is one of the materials in group 1. Table 2-6 lists some of the principal applications of various alloys for different applications [21]. ASTM is an abbreviation of American Society for Testing and Materials. 17-4PH is remarkable for its outstanding performance for rotary valves and pressure feeders. In general, Stellite alloy and stainless steel are two common types of materials used for feed water valves [22].

Table 2-5 Relative erosion/abrasion resistance of various alloys [21]

\begin{tabular}{|c|c|c|c|}
\hline Group & Alloy & Hardness & Erosion/abrasion \\
\hline \multirow{2}{*}{ 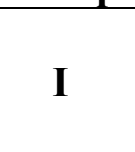 } & $28 \% \mathrm{Cr}$ white iron & $600 \mathrm{Bhn} 59 \mathrm{HRC}$ & Excellent \\
\hline & $\begin{array}{c}\text { Stellite } 6 \text { Co-base hard facing } \\
\text { alloy }\end{array}$ & 45-50HRC & \\
\hline \multirow{3}{*}{ II } & & Tensile strength MPa & \\
\hline & Precipitation hardening alloys & 860 & \\
\hline & Martensitic stainless steels & $620-900$ & \\
\hline III & Duplex stainless steels & $620-690$ & \\
\hline \multirow{3}{*}{ IV } & $\mathrm{CU}-5 \mathrm{M} \mathrm{CuC}$ & 520 & \\
\hline & Superaustenitic & 550 & \\
\hline & CG-3M/CG-8M & $515-520$ & \\
\hline $\mathbf{V}$ & CF-3/CF-8 and CF-3M/CF-8M & 485 & Poor \\
\hline
\end{tabular}


Table 2-6 Common usage of cast stainless steels [21]

\begin{tabular}{|c|c|c|c|c|}
\hline NO. & Cast grade & $\begin{array}{c}\text { ASTM } \\
\text { specification }\end{array}$ & $\begin{array}{l}\text { Wrought } \\
\text { equivalent }\end{array}$ & Usage \\
\hline \multicolumn{5}{|c|}{ White cast irons } \\
\hline 1 & $28 \% \mathrm{Cr}$ & A523 IIIA & none & Refiner discs and pumps \\
\hline \multicolumn{5}{|c|}{ Martensitic } \\
\hline 2 & CA-15 & A487 & S41000 & $\begin{array}{l}\text { Boiler feed water pumps, mechanical } \\
\text { pulping equipment, feeders for } \\
\text { continuous digesters }\end{array}$ \\
\hline 3 & CA-6NM & A487 & S41500 & Upgrade from 2, preferred. \\
\hline 4 & CB-6 & A743 & $\mathrm{SS} 2387^{(2)}$ & Mechanical pulping equipment \\
\hline & 16Cr5Ni1Mo & $\mathrm{SS} 2387^{(2)}$ & DIN $1.4418^{(1)}$ & \\
\hline & DIN $1.4405^{(1)}$ & DIN $1.4418^{(1)}$ & & \\
\hline \multicolumn{5}{|c|}{ Precipitation hardening } \\
\hline 5 & $\mathrm{CB}-7 \mathrm{Cu}-1$ & A747 & $17-4 \mathrm{PH}$ & $\begin{array}{c}\text { Liners for rotary valves and pressure } \\
\text { feeders }\end{array}$ \\
\hline 6 & $\mathrm{CB}-7 \mathrm{Cu}-2$ & A747 & $15-5 \mathrm{PH}$ & $\begin{array}{c}\text { Refiner discs; other mechanical pulping } \\
\text { equipment }\end{array}$ \\
\hline 7 & $\mathrm{CD}-6 \mathrm{MN}(3 \mathrm{~A})$ & A890 & $\begin{array}{c}329 \\
(\mathrm{SS} 2324)^{(2)}\end{array}$ & Same as 8 \\
\hline 8 & $\begin{array}{l}\text { CD-4MCuN } \\
\text { (1B) }\end{array}$ & A 890 & $x_{1}$ & $\begin{array}{l}\text { Alternative to } 10-17 \text { below, preferred } \\
\text { when sand or other abrasives are present }\end{array}$ \\
\hline 9 & J92205 & A890 & 2205 & Same as 8 \\
\hline 10 & CF-3 & A743/A744 & $304 \mathrm{~L}$ & Digester blow valves, stock pumps in \\
\hline 11 & $\mathrm{CF}-8$ & A743/A744 & 304 & $\begin{array}{c}\text { clean water, kraft liquor and mill } \\
\text { effluent }\end{array}$ \\
\hline 12 & CF-3M & A743/A744 & $316 \mathrm{~L}$ & $\begin{array}{l}\text { White water, chemical recovery, milder } \\
\text { bleach }\end{array}$ \\
\hline 13 & CF-8M & A743/A744 & 316 & $\begin{array}{c}\text { plant services, \& kraft liquor \& mill } \\
\text { effluent }\end{array}$ \\
\hline 14 & CG-3M & A743/A744 & $317 \mathrm{~L}$ & $\begin{array}{c}\text { Bleach plant services except most } \\
\text { aggressive }\end{array}$ \\
\hline 15 & CG-8M & A743/A744 & 317 & chlorine and chlorine dioxide services \\
\hline 16 & $\mathrm{CN}-7 \mathrm{M}$ & A743/A744 & N08020 & Alternative to CF-8M for valves \\
\hline 17 & CU-5MCUC ${ }^{(3)}$ & A494 & N08825 & Chip injectors for continuous digesters \\
\hline \multicolumn{5}{|c|}{ Superaustenitic } \\
\hline 18 & $\mathrm{CK}-3 \mathrm{MCuN}$ & A743/A744 & S31254 & $\begin{array}{l}\text { Aggressive chlorine and chlorine } \\
\text { dioxide bleach plant services }\end{array}$ \\
\hline 19 & $\mathrm{CN}-3 \mathrm{MN}$ & A743/A744 & N08637 & $\begin{array}{l}\text { Aggressive chlorine and chlorine } \\
\text { dioxide bleach plant services }\end{array}$ \\
\hline 20 & $654 \mathrm{SM}^{(4)}$ & - & S32654 & $\begin{array}{l}\text { Aggressive chlorine dioxide bleach } \\
\text { plant services }\end{array}$ \\
\hline \multicolumn{5}{|c|}{$\begin{array}{l}\text { (1) German alloy } \\
\text { (2) Swedish alloy designation } \\
\text { (3) Contains } 1.0 \mathrm{Nb} \\
\text { (4) Designation not yet assigned. }\end{array}$} \\
\hline
\end{tabular}




\subsubsection{Stellite alloy family}

Stellite alloy refers to a family of alloys that contain at least $50 \mathrm{wt} \%$ cobalt, which is popularly used in various industries, such as jet engine turbine blades in gas turbine engine operation, valves in oil production and refining or power generation, cutting tools in mechanical manufacturing and high corrosion-resistant parts of machinings. Stellite alloys have a good combination of high hardness and strength, good adhesive and abrasive wear resistance and good resistance to erosion in all of these industries [23]. Actually, this is a trademarked name of Kennametal Stellite Inc., and Stellite alloy is known for its superior wear resistance [24]. The composition of Stellite alloy differs from alloy to alloy, but the main alloying elements of these alloys are similar. Cobalt, nickel, iron, aluminum, boron, carbon, chromium, manganese, molybdenum, phosphorus, sulfur, silicon and titanium may compose different types of Stellite alloys. However, four to six of these elements with different proportions may constitute a specific Stellite alloy.

Table 2-7 summarizes the chemical compositions of commonly used Stellite alloys [25]. It is obvious that most of the Stellite alloys contain 20 to $30 \mathrm{wt} \% \mathrm{Cr}, 5$ to $15 \mathrm{wt} \% \mathrm{~W}$ or Mo and 0.1 to $3 \mathrm{wt} \% \mathrm{C}$. The classification of Stellite alloy family comes from the weight percentages of carbon and tungsten/molybdenum contents, which affect the carbide formation and microstructure [25]. The carbides at grain boundaries and in the Co matrix highly affect the strength, corrosion resistance and hardness of Stellite alloys, whereas the tantalum, tungsten, molybdenum, chromium, and columbium decide the strengthening capacity of the Co solid solution [26]. 
Table 2-7 Compositions (wt\%, Co in balance) of various Stellite alloys [25]

\begin{tabular}{|c|c|c|c|c|c|c|c|c|c|c|}
\hline Alloy & Process & Cr & W & Mo & C & Fe & Ni & Si & Mn & Others \\
\hline Stellite 1 & Cast & 30 & 13 & 0.5 & 2.5 & 3 & 1.5 & 1.3 & 0.5 & \\
\hline Stellite 3 & P/M & 30.5 & 12.5 & & 2.4 & 5 & 3.5 & 2 & 2 & $1 \mathrm{~B}$ \\
\hline Stellite 4 & Cast & 30 & 14 & 1 & 0.57 & 3 & 3 & 2 & 1 & \\
\hline Stellite 6 & Cast & 29 & 4.5 & 1.5 & 1.2 & 3 & 3 & 1.5 & 1 & \\
\hline Stellite 6 & P/M & 28.5 & 4.5 & 1.5 & 1 & 5 & 3 & 2 & 2 & $1 \mathrm{~B}$ \\
\hline Stellite 12 & Cast & 30 & 8.3 & & 1.4 & 3 & 1.5 & 0.7 & 2.5 & \\
\hline Stellite 21 & Cast & 27 & & 5.5 & 0.25 & 3 & 2.75 & 1 & 1 & \\
\hline Stellite 20 & Cast & 33 & 17.5 & & 2.45 & 2.5 & 2.5 & & 1 & \\
\hline Stellite 22 & Cast & 27 & & 11 & 0.25 & 3 & 2.75 & 1 & 1 & \\
\hline Stellite 25 & Cast & 20 & 15 & & 0.1 & 3 & 10 & 1 & 1.5 & \\
\hline Stellite 31 & Cast & 22 & 7.5 & & 0.5 & 1.5 & 10 & 0.5 & 0.5 & \\
\hline Stellite 80 & Cast & 33.5 & 19 & & 1.9 & & & & & $1 \mathrm{~B}$ \\
\hline Stellite 188 & Cast & 22 & 14 & & 0.1 & 3 & 22 & 0.35 & 1.25 & $0.03 \mathrm{La}$ \\
\hline Stellite 190 & Weld & 27 & 14 & 1 & 3.3 & 3 & 3 & 1 & 1 & \\
\hline Stellite 300 & Cast & 22 & 32 & & 1.5 & & & & & \\
\hline Stellite 694 & Cast & 28 & 19 & & 1 & 5 & & 1 & 1 & \\
\hline Stellite 712 & Cast & 29 & & 8.5 & 2 & 3 & 3 & 1.5 & 1.5 & \\
\hline Stellite 720 & Cast & 33 & & 18 & 2.5 & 3 & 3 & 1.5 & 1.5 & $0.3 \mathrm{~B}$ \\
\hline Stellite 703 & Cast & 32 & & 12 & 2.4 & 3 & 3 & 1.5 & 1.5 & \\
\hline Stellite 706 & Cast & 29 & & 5 & 1.2 & 3 & 3 & 1.5 & 1.5 & \\
\hline Stellite 706K & Wrought & 29 & & 6 & 1.4 & 3 & 3 & 1.5 & 1.5 & \\
\hline Stellite 6B & Wrought & 30 & 4 & 1.5 & 1 & 3 & 2.5 & 0.7 & 1.4 & \\
\hline Stellite 6K & Wrought & 30 & 4.5 & 1.5 & 1.6 & 3 & 3 & 2 & 2 & \\
\hline Stellite 98M2 & P/M & 30 & 18.5 & 0.8 & 2 & 5 & 3.5 & 1 & 1 & $4.2 \mathrm{~V}, 1 \mathrm{~B}$ \\
\hline
\end{tabular}

\subsubsection{Previous research on Stellite 6 alloy}

Stellite 6 alloy is the first alloy of the Stellite alloy family, developed by Elwood Haynes in 1900. The approximate chemical composition of Stellite 6 alloy is Co28Cr4.5W1.1C (in weight) [27]. Because of its good all-round performance, it becomes one of the most widely used Stellite alloys. Consequently, lots of studies on this alloy have been reported.

For example, the aqueous oxidation of Stellite 6 alloy was studied using X-ray photoelectron spectroscopy (XPS) method in 1979 [28]. The specimen was put into water 
$(\mathrm{pH}=10)$ at $285^{\circ} \mathrm{C}$. A function of dissolved oxygen concentration with length of exposure time was measured to investigate the aqueous oxidation behavior of Stellite 6 alloy.

In 1985, Hocking et al. studied the corrosion mechanisms of Stellite 6 in lithiated high-temperature water [29]. They tried to simulate the operation of a pressurized water reactor coolant circuit in the lab to study the corrosion behavior of Stellite 6 alloy in lithiated high-temperature water. The XPS method was employed to obtain the chemical composition of the corrosion layer, and a Scanning Auger Microscopy was used to determine the spatial distributions of elements within the oxide layer and alloy matrix.

The wear resistance of Stellite 6 alloy with the change of microstructure was investigated by Frenk and Kurz in 1994 [27]. A wide range of solidification microstructures of Stellite 6 alloy were obtained via casting and laser cladding methods, and the specimens with different microstructures were tested under dry sliding wear conditions. Based on the experimental results, Frenk and Kurz suggested that Stellite 6 alloy was capable of serving in such severe metallic wear conditions.

The wear behavior of lased-clad stellite 6 alloy was studied in 1996 [30]. The specimens were made with laser cladding Stellite 6 powder on mild and medium carbon steels, and they were then tested on a pin-on-disc tribometer. SEM examination and X-ray diffraction analysis were performed on the worn surfaces of the laser-clad Stellite 6 alloy specimens under various controlled loads and sliding speeds. 
In 1997 and 1998, the investigation in the microstructure and mechanical properties of Stellite 6 alloy with some additives was made by Kuzucu et al. [31]. They studied the change in properties of Stellite 6 alloy as a result of adding silicon to $6 \mathrm{wt} \%$ [32]. In the research of Kuzucu et al., the metallographic, X-ray diffraction techniques and thermal analysis methods were employed, and hardness tests were performed on Stellite 6 for the phase analyses.

A comparison study in the performance of Stellite 6 processed via different techniques in liquid-solid slurries was made by Malayoglu and Neville in 2003 [33]. The specimens were prepared via Hot Isostatic Press (HIP) and cast process, respectively, and they then underwent liquid-solid erosion tests at the same temperature $\left(20^{\circ} \mathrm{C}\right.$ and $\left.50^{\circ} \mathrm{C}\right)$. Two stainless steels (UNS S32760 and UNS S31603) were tested under the same temperature and liquid-solid loading conditions. UNS refers to Unified Numbering System. It was shown that the HIPed Stellite 6 alloy had the best erosion and corrosion resistance among these test alloys.

In 2005, erosion-corrosion test was performed on Stellite 6 alloy and Stellite 706 alloy by Malayoglu et al., which were fabricated via cast and HIP process, respectively [34]. Malayoglu et al. also studied the kinetics and mechanisms of the corrosion of cast and HIPed Stellite 6 in aqueous saline $(3.5 \% \mathrm{NaCl})$ environments in the same year [35]. A comparison in corrosion behavior between cast Stellite 6 alloy and HIP consolidated Stellite 6 alloy was made using DC (Direct Current) electrochemical methods in static saline circumstances. It was shown that the significant difference in the corrosion 
performance between cast Stellite 6 alloy and HIP consolidated Stellite 6 alloy was associated to the different microstructures resulting from the two processing methods.

Wear, corrosion and cracking resistances of some $\mathrm{W}$ or Mo containing Stellite alloy hardfacings were reported by Yao et al. in 2005 [36]. A series of tests were conducted to investigate the abrasive, adhesive and erosive wear resistances of these alloy hardfacings. Scratch test was also performed on these alloy hardfacings to evaluate the critical plastic shear strain, which was associated with the cracking resistance of the materials.

In 2008, Chang et al. investigated the impact wear resistance of Stellite 6 hard-faced valve seats via plasma transferred arc and laser cladding, respectively [37]. The advantages of increased hardness, impact toughness, and superior impact wear resistance were found on the laser-clad specimens.

Thermal fatigue tests were performed on Inconel 647 and Stellite 6 alloys as potential tooling materials by Birol in 2010 [38]. He also tested the thermal fatigue of the hot work tool steel when Stellite 6 alloy was used as a coating [39]. The Cr content in Stellite 6 alloy allowed the base material to survive longer because Cr-rich oxides can form on the alloy so that the thermal stresses at the surface can be arrested. As a result, the crack initiation can be retarded.

Gholipour et al. conducted the microstructure and the wear experiments of Stellite 6 laser cladding on 17-4PH stainless steel in 2011 [40]. The phases formed in the coating were 
identified using X-ray diffraction analysis. The microstructure and wear mechanisms of the Stellite 6 laser cladding were studied using optical metallography, SEM/ EDX.

In 2012, Romo et al. tested welded Stellite 6 alloy to investigate the cavitation and high-velocity slurry erosion resistances [41]. The ASTM G32 standard was used for instructing the cavitation resistance experiments. A high-velocity erosion tester was used with different impact angles for the slurry erosion resistances measurements.

\subsubsection{Previous research on 17-4PH stainless steel}

$17-4 \mathrm{PH}$ stainless steel is a very popular martensitic precipitation-hardening stainless steel with a chemical composition of $\mathrm{Fe} 15.8 \mathrm{Cr} 3.8 \mathrm{Cu} 3.8 \mathrm{Ni}$ (in weight). It exhibits a superior combination of high strength, good corrosion resistance, and good mechanical properties $[42,43]$. Additionally, the heat treatment processes are relatively easy for $17-4 \mathrm{PH}$ to gain high strength and excellent corrosion resistance [44]. However, the limitations of 17-4PH stainless steel are the relatively low hardness and poor tribological properties [45].

The strength, fracture toughness and low cycle fatigue behavior of $17-4 \mathrm{PH}$ stainless steel were studied by Rack and Kalish in 1974 [46]. They tried to find a correlation between the fatigue crack growth rate and the monotonie tensile property, but it was unsuccessful. They finally found a relationship between the final failure and the critical strain criteria, thus the preferential void nucleation and growth at $\delta$-ferrite-matrix interfaces could reasonably describe the fracture toughness. 
In 1988, Viswanathan et al. investigated the effects of aging on the microstructure of 17-4PH stainless steel [47]. Viswanathan et al. studied the microstructures of 17-4PH stainless steels as the results of various heat treatments. Then the discussion was made on the morphologies of the transformation products and the crystallography of the transformation. Viswanathan et al. also did the experiments for the kinetics of precipitation in 17-4PH stainless steel in 1989 [48]. The electrical resistivity and microstructure of the $17-4 \mathrm{PH}$ stainless steel were investigated to study the sequence of precipitation and its kinetics for 17-4PH stainless steel.

Murayama et al. analyzed the microstructures of $17-4 \mathrm{PH}$ stainless steel at various stages of heat treatment in 1998 [49]. The atom probe field ion microscopy and transmission electron microscopy were used to study the microstructures of $17-4 \mathrm{PH}$ stainless steels after solution heat treatment, tempering at $580^{\circ} \mathrm{C}$, and long-term aging at $400^{\circ} \mathrm{C}$.

Hsiao et al. investigated the aging reactions on the microstructure development in 17-4PH stainless steel using transmission electron microscopy in 2002 [50].

The research in activated gas nitriding of $17-4 \mathrm{PH}$ stainless steel was performed by Kochmański and Nowacki in 2006 [51]. The 17-4PH stainless steel specimens were nitrided in the supersaturated or precipitation-hardened state, and then SEM, EDX, and wavelength dispersive X-ray spectroscopy, and X-ray diffraction were utilized to study the nitrided layers. Although the same nitriding conditions were used, the differences in 
surface morphology, thickness, phase composition and nitrogen concentration of the diffusion layers were found on 17-4PH stainless steels.

Ping et al. published an article about the effect of aging temperature on the erosion-corrosion behavior of 17-4PH stainless steels in dilute sulphuric acid slurry [52]. Before the tests, different aging temperatures were chosen for the 17-4PH stainless steel specimens. They conducted these tests on a self-made erosion-corrosion apparatus and used SEM to analyze the surface micrographs of the specimens after the erosion-corrosion tests.

In 2008, the influence of hardness on the wear resistance of $17-4 \mathrm{PH}$ stainless steel was investigated by Bressan et al. using the pin-on-disc test method [53]. The precipitation hardening and the aging method allowed the steel to reach various hardness levels. SEM was also used to study the worn surfaces of the steel specimens to find the wear mechanisms.

Hot compression test was performed for the flow curve analysis of $17-4 \mathrm{PH}$ stainless steel by Mirzadeh et al. in 2009 [54]. They tried to find if the dynamic recrystallization phenomenon occurred in $17-4 \mathrm{PH}$ stainless steel in its work hardening rate analysis. Moreover, they also studied experimentally the aging kinetics of 17-4PH stainless steel in the same year [55]. The artificial neural networks method was used for modeling and analyzing the effects of aging temperature and time with regard to tempering parameters on the strengthening behavior of $17-4 \mathrm{PH}$ stainless steel. It was found that precipitation rate 
exponentially depended on aging temperature. The precipitation kinetics of this martensitic aging hardenable alloy was characterized via the Johnson-Mehl-Avrami-Kolmogorov analysis.

In 2012, Murr et al. conducted experiments on the microstructures and properties of 17-4PH stainless steel using the selective laser melting method [56]. The samples of 17-4PH stainless steel were fabricated via the selective laser melting method in nitrogen or argon atmosphere. Optical microscopy, SEM, X-ray diffraction, and transmission electron microscopy were employed to investigate the microstructures and phase structures.

The research in the hot deformation and dynamic recrystallization of 17-4PH stainless steel was published by Mirzadeh and Najafizadeh in 2013 [57]. The effects of deformation conditions on the occurrence of dynamic recrystallization and on the final grain size were revealed through developing a dynamic recrystallization map. This research was much similar to the research of Mirzadeh et al. using the same compression test in 2009 [54].

In 2014, Nakhaie and Moayed studied the pitting corrosion of cold rolled solution treated 17-4PH stainless steel [58]. The effect of cold rolling on the pitting of 17-4PH stainless steel was investigated. The transition from metastable to stable pitting was facilitated by cold rolling and the cold working experienced an increasing trend when the frequency of metastable pitting decreased, but there was no significant change in the pitting potential distribution as a result of cold working. 


\subsubsection{Summary of previous research on Stellite 6 alloy and 17-4PH stainless steel}

The wear behavior studies of 17-4PH stainless steel and Stellite 6 have been reported extensively. There were also lots of studies in the microstructure and material properties of 17-4PH stainless steel as well as aging reactions or kinetics. As for Stellite 6 alloy, the researches in microstructures and material properties as a result of the addition of other alloy elements have been found largely in literature. The fatigue behavior of Stellite 6 alloy and 17-4PH stainless steel were also the interests of many researchers. The corrosion problems of 17-4PH stainless steel in acid [52] or neutral environments [45] were reported. The corrosion experiments of Stellite 6 alloy were made in water [29], $\mathrm{NaCl}$ solution [34, 35] and acid solution [36]. However, the studies of these materials in amine environments were rarely reported.

\subsection{Methods of corrosion study}

The methods of investigating the corrosion behavior of materials can be divided into three categories: electrochemical method, immersion test method, and optical analysis. Electrochemical method refers to a series of analytical chemistry techniques measuring the potential or current of the electrochemical cell. Immersion test method is known as a traditional approach for studying the corrosion behavior of materials. The corrosive medium, $\mathrm{pH}$ value, test temperature, exposure time and pressure may be controlled as the selective variables to simulate or accelerate the real work environments [23]. SEM, EDX, X-ray diffraction and XPS are the main methods included in optical analysis technologies. 
Two of them are often utilized together to analyze the materials according to elements and microstructures [23].

\subsubsection{Fundamentals of electrochemical corrosion test}

High sensitivity, accuracy, precision, large linear dynamic range and relatively low cost for instrumentation are the main advantages of electrochemical methods [59]. Extraordinary progress has been made in the electrochemical analysis. Monitoring the electron flow as a measuring process is the fundament of electrochemical testing because corrosion is an electrochemical reaction and the current flowing between anodic and cathodic areas determines the corrosion rate [60]. Polarization methods, including potentiodynamic polarization, polarization resistance, cyclic polarization, EIS method, electrochemical noise measurement, potentiostatic and galvanostatic methods, are popular in corrosion analysis [61].

In this thesis, potentiodynamic polarization, cyclic polarization and EIS methods were chosen together to study the corrosion behavior of Stellite 6 alloy and 17-4PH stainless steel in amine environments. Electrochemical tests commonly run in three-electrode mode [62]: a Working Electrode (WE) or material sample and a graphite rod used as the Counter Electrode (CE) and a Saturated Calomel Electrode (SCE) used as a Reference Electrode (RE) work together to compose a common three-terminal electrochemical cell, as shown in Figure 2-4 [63]. 


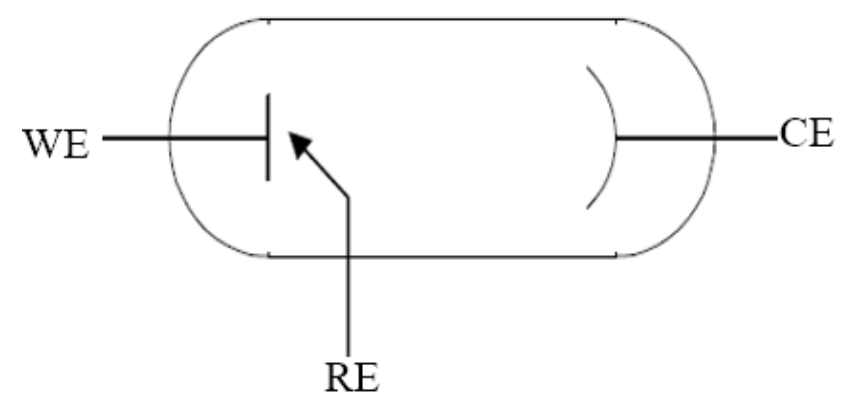

Figure 2-4 Three-terminal electrochemical cell [63]

\subsubsection{Potentiodynamic polarization}

Potentiodynamic polarization method is a representative of the DC polarization technique. It is performed in a wide range of use in obtaining the corrosion rate, pitting susceptibility, passivity and the cathodic behavior of an electrochemical system. As well known, an anode, a cathode, an ionic and an electrical conduction path between the anode and the cathode are four primary conditions for the electrochemical process. The WE, CE and the solution between them create the ionic conduction path, whereas the potentiostat provides the electrical path. In this way, the potentiostat can control and dictate the electrochemical process. As the electrons flow through the electrical path from the anode to the cathode, the anode and cathode are generally regarded as the negative electrode and the positive electrode respectively. The reaction rate of the WE is represented by the current. The current density, which is expressed as the current per unit area of the WE, is commonly used [64].

Oxidation takes place at the anode, and the oxidation process in metals can be described by Equation 2-1: 


$$
M \rightarrow M^{x+}+x e^{-}
$$

where $M$ represents the alloy or the pure metal, $M^{x+}$ represents the ion of the metal and $x e^{-}$represents how many electrons can be transferred in the process.

However, metals are not the only materials that can be oxidized, other chemical species in that environment may also be oxidized. For instance, ferrous ions can be oxidized to ferric ions $\mathrm{Fe}^{2+}$ or $\mathrm{Fe}^{3+}$ in iron-containing solutions. In aqueous environments, oxygen products may also be obtained and this process is described by Equation 2-2:

$$
2 \mathrm{OH}^{-} \rightarrow 1 / 2 \mathrm{O}_{2}+\mathrm{H}_{2} \mathrm{O}+2 e^{-}
$$

On the other hand, reduction takes place at the cathode. The oxide or hydroxide on the cathode surface may experience the reduction process, as expressed by Equation 2-3:

$$
M_{x}(O H)_{y}+y e^{-} \rightarrow x M+y O H^{-}
$$

Similar to the oxidation process, the reduction reaction occurs not only on the oxides or hydroxides present on that electrode, but also water reduction may occur in aqueous environments at sufficiently negative potentials through Equation 2-4:

$$
\mathrm{H}_{2} \mathrm{O}+e^{-} \rightarrow 1 / 2 \mathrm{H}_{2}+\mathrm{OH}^{-}
$$

A typical hypothetical polarization curve obtained using the electrochemical measurement method is found in Figure 2-5 (a). The absolute value of the current density is plotted in logarithm with base 10 .

It is shown that the polarization curve starts at point 1 in the cathodic region and ends at point 2. The point $A$ indicates the open circuit potential (OCP) or corrosion potential $E_{c o r r}$ 
where the sum of the anodic and cathodic reaction rates is zero on the electrode surface. The region $B$ is the active region where the dominant reaction is metal oxidation. As the applied potential keeps increasing, the passivation potential $E_{p p}$ and critical current density $i_{c}$ (point $C$ ) are reached. After this point, the region $D$ experiences a decrease in current density when the potential increases until the scan goes into the passive region $E$ where the passive current density $i_{p}$ is very low and stable when the passive potential $E_{p a}$ increases. Point $F$ is referred to breakaway potential $E_{b}$ or pitting potential $E_{p i t}$. After this point, region $G$ demonstrates the region where the applied current increases dramatically. This increase can be related to the pitting phenomenon (localized breakdown of passivity) [64]. Some relevant parameters from polarization curve are given in Figure 2-5 (b). However, it should be noticed that the real potentiodynamic polarization curve may include some but not all of the features of the typical hypothetical polarization curve.

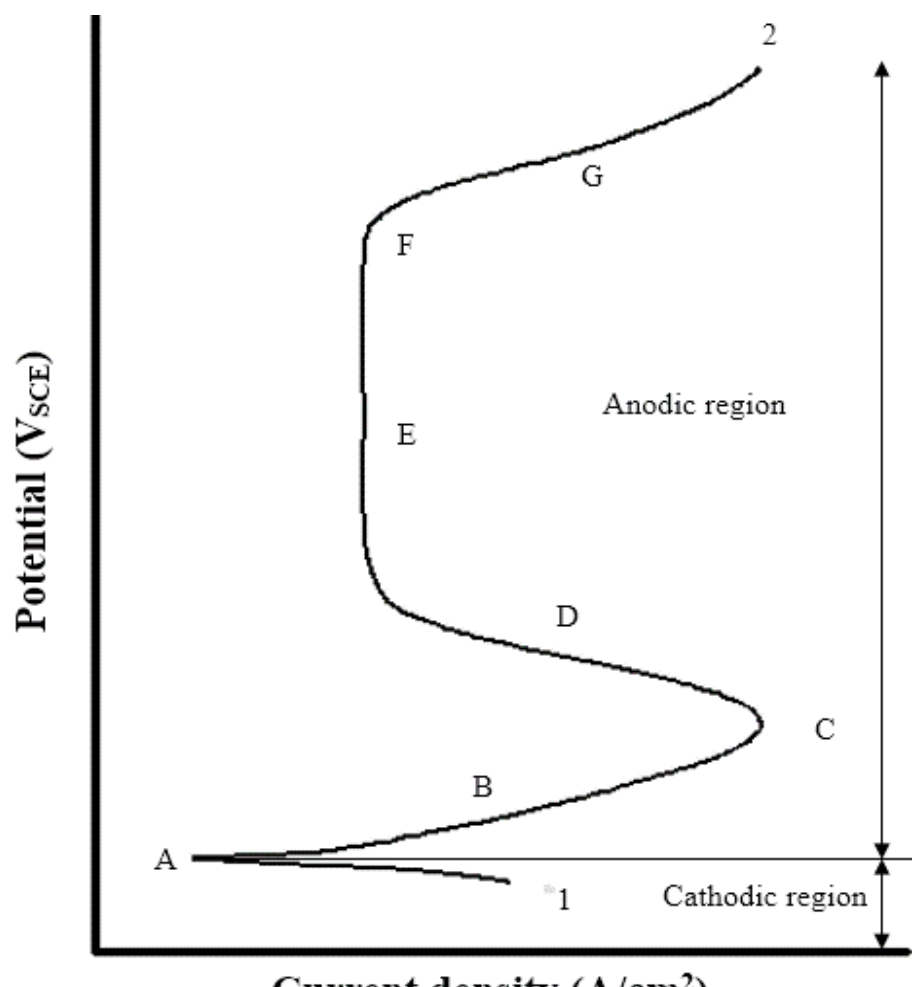

Current density $\left(\mathrm{A} / \mathrm{cm}^{2}\right)$

(a) 


\begin{tabular}{|c|c|}
\hline & Meaning \\
\hline $\mathbf{i}_{\mathbf{p}}$ & Passive current density \\
\hline $\mathbf{i}_{\mathbf{c o r r}}$ & Corrosion current density \\
\hline $\mathbf{i}_{\mathbf{c}}$ & Critical current density \\
\hline $\mathbf{E}_{\mathbf{p a}}$ & Passive potential \\
\hline $\mathbf{E}_{\mathbf{c o r r}}$ & Corrosion potential \\
\hline $\mathbf{E}_{\mathbf{p p}}$ & Primary passive potential \\
\hline $\mathbf{E}_{\mathbf{b}}$ & Breakaway potential \\
\hline
\end{tabular}

(b)

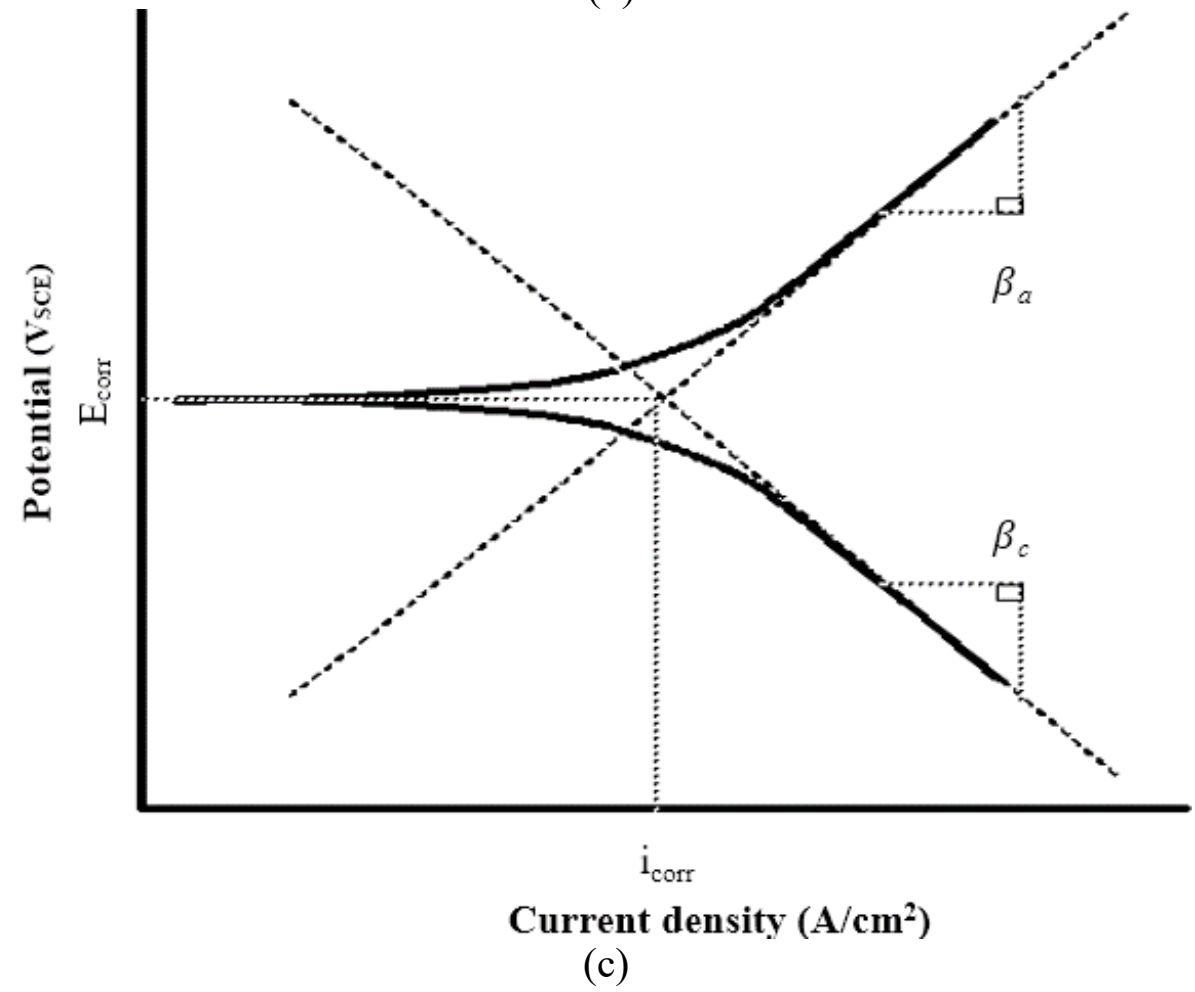

Figure 2-5 Typical polarization curves: (a) anodic polarization curve; (b) parameters related to polarization curve (c) Tafel slope [64]

The anodic and cathodic regions are two of the main regions of a polarization curve, as indicated in Figure 2-5 (c). The $\beta_{a}$ and $\beta_{c}$ are the anodic slope and cathodic slope respectively. They can be used in Tafel extrapolation method for further calculation. The corrosion potential $E_{c o r r}$, current density $i_{c o r r}$ and polarization resistance $R_{p}$ can be calculated using the Tafel extrapolation method [23]. The details of Tafel extrapolation method are given below: 
(1) Stern-Geary Equation 2-5 can be used to calculate the corrosion current $i_{\text {corr }}[65]$ :

$$
i_{\text {corr }}=\frac{\beta_{a} \beta_{c}}{2.3026\left(\beta_{a}+\beta_{c}\right) R_{P}}
$$

(2) The Tafel slopes $\beta_{a}$ and $\beta_{c}$ correspond to the anodic and cathodic regions, respectively, and can be obtained from the Tafel diagram directly.

(3) The parameter $B$ represents the Stern-Geary constant and can be calculated using Equation 2-6:

$$
B=\frac{\beta_{a} \beta_{c}}{2.3026\left(\beta_{a}+\beta_{c}\right)}
$$

(4) The polarization resistance $R_{p}$ can be obtained through the Equation 2-7:

$$
R_{p}=\frac{B}{i_{c o r r}}
$$

Thus, the corrosion behavior of the material can be predicted from the potentiodynamic polarization curve. A quicker passivation and better corrosion resistance of the material can be deduced from a lower calculated current density [66].

\subsubsection{Cyclic polarization}

Cyclic polarization is similar to potential dynamic polarization. The main difference between them is that the cyclic polarization curve has a reverse scan until a predetermined value of potential is reached, and then the reverse scan may stop at another predetermined value. However, these predetermined values change in different experimental environments for different metals. The reverse scan can be named as hysteresis loop, and the existence of hysteresis is usually the indicator of pitting, while the size of the loop is 
often related to the amount of pitting. A large difference in hysteresis loop can be seen in Figure 2-6 as an example [67].

For these curves, the protection potential $E_{\text {prot }}$ demonstrates the potential where the passivity may restore after its breaking down. There is a large hysteresis loop on the curve of stainless steel 317L, but none or little can be seen for alloy G-3. A larger protection potential $E_{\text {prot }}$ and a smaller difference between breakaway potential $E_{b}$ and $E_{\text {prot }}$ always indicate better resistance to localized corrosion [66].

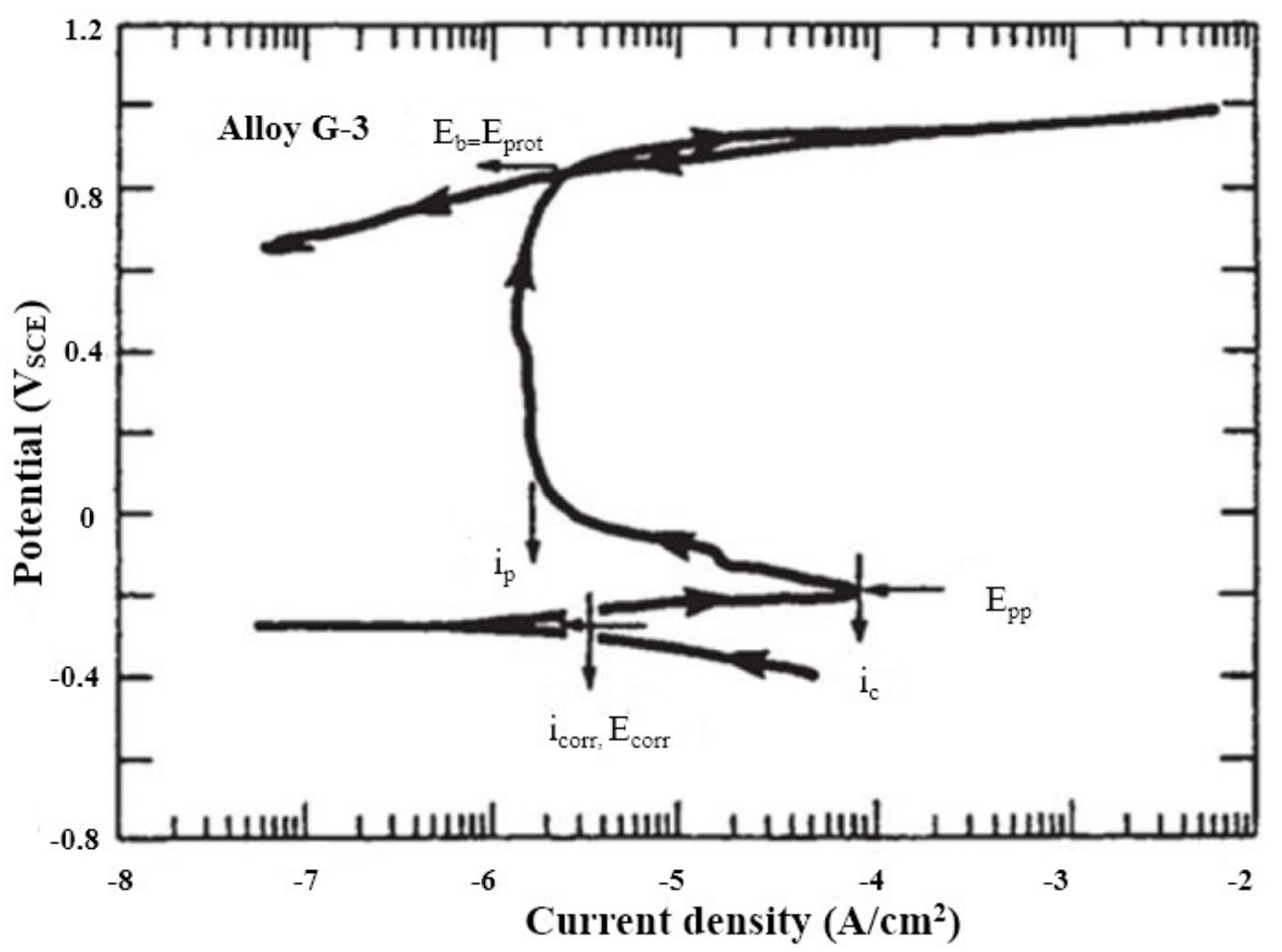

(a) 


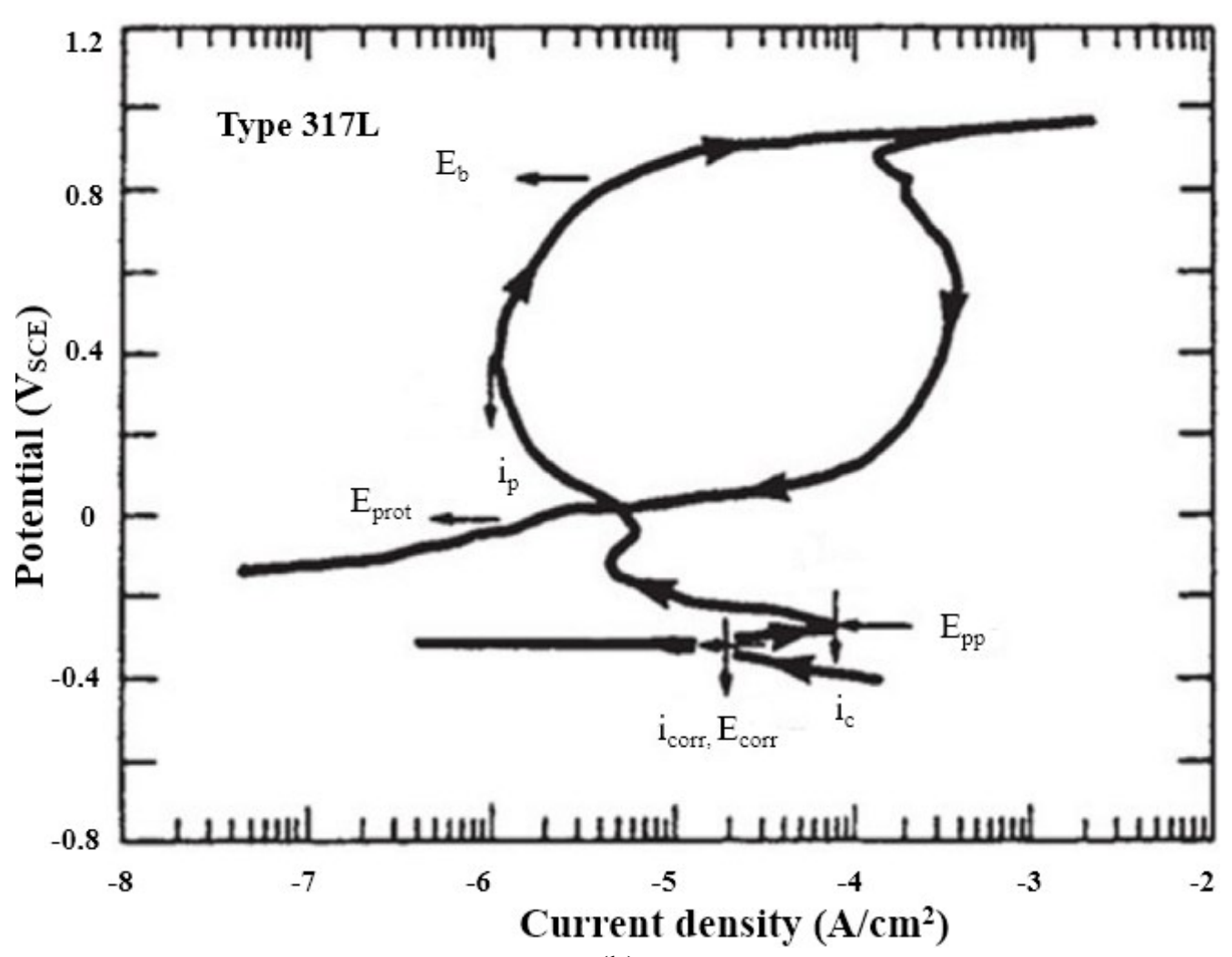

(b)

Figure 2-6 Cyclic polarization curves tested in $\mathrm{pH} 1$ sulfuric acid solution with 10,000 ppm chlorine, $1000 \mathrm{ppm}$ fluorine, and $5000 \mathrm{ppm}$ manganese at $50^{\circ} \mathrm{C}$ for: (a) Alloy G-3; (b) Type 317L [67]

\subsubsection{EIS}

A current will flow through the cell if a potential is applied to an electrode system. The movement of ions through the electrolyte leads to the formation of new chemical species. If the applied potential is a sinusoid $(\Delta E \cdot \sin \omega t)$, the Alternating Current (AC) resulting from the potential will also be sinusoidal $(\Delta i \cdot \sin \omega t)$, and the impedance is known as the relationship between the applied potential and the current flow. Moreover, this relationship is similar to the resistance-current-potential relationship of a DC circuit (Ohm's law). The impedance $(Z)$ has a magnitude $(\Delta \mathrm{E} / \Delta \mathrm{i})$ and phase $(\varphi)$ and is thus a vector quantity [63]. Therefore, EIS is the result of a series of impedance $(Z)$ with a series of different phases 
$(\varphi)$. However, EIS must satisfy the three basic conditions: the causality condition, the linear condition and the stability condition.

\subsubsection{Fundamental requirements}

The causality, the linearity, and the stability are three fundamental requirements of EIS.

When the sinusoidal potential is applied on the electrode system, the electrode system must respond only to that potential signal according to the causality requirement. Thus, all the other state variables and the potential of the electrochemical cell must change accordingly. In the circuit consisting of the electrical components, there is only one state variable. However, in the circuit consisting of the electrochemical components, there are a number of state variables, such as the concentration of the reactant, the temperature of the environment, and so on. To meet the requirements of the causality requirement between the response and the applied signal, the state variables must be controlled [68].

Because of the kinetics of the electrode process, the relationship between the change rate of the electrode process and the state variable does not exhibit linearity. The relationship between the change of the electrode process rate and the state variable can be considered linear only when the change of the state variable is small enough. In order to make sure that the linearity requirement is satisfied, the amplitude of the sinusoidal potential excitation must be very small. Thus the linearity can be found between the response signal and the excitation signal similarly [68]. 
The stability requirement refers to whether the electrode system can restore to its original state when the excitation stops or does not have a close relationship with the internal structure of the electrode system, that is, the kinetics of the electrode process. When the excitation is applied on the electrode system, there is no significant change in the internal structure, the electrode system can return to its original state.

This electrode process is similar to the situation shown in Figure 2-7 (a). The ball stays at bottom forever if there are no external forces. When the external force is not too large, the ball is on the slide. When the external force disappears, the ball returns to the bottom. However, if the electrode process is similar to the situation shown in Figure 2-7 (b). When the external force is too large, or the boundary between the two valleys is not very high, the ball could go across the boundary and fall into the other valley, thus the original state cannot be maintained. An irreversible electrode process may lead to this similar situation easily. For instance, anodic dissolution and cathodic reduction processes can occur simultaneously on the surface of the corroded metal electrode. As a result, the surface of the electrode changes constantly. Of course, if the change on the electrode surface is not too fast the amplitude of the excitation is small, the operation time is not long, and the electrode system could return to the situation which is very similar to the original state, the stability requirement can be considered satisfied approximately. 


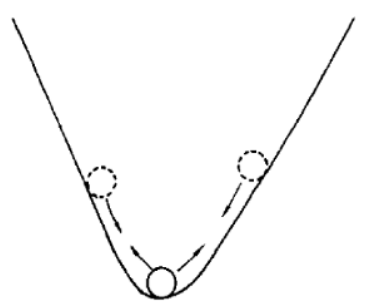

(a)

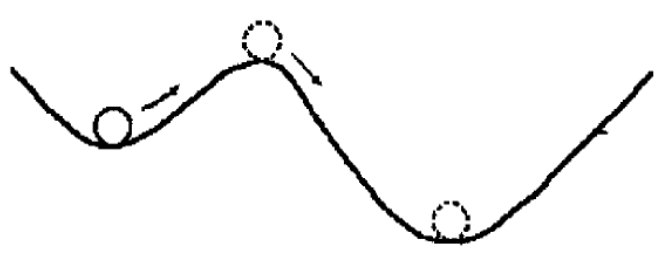

(b)

Figure 2-7 Structures used to simulate the electrode process: (a) steady structure; (b) unsteady structure [68]

\subsubsection{Equivalent circuits and components}

The EIS of the electrode system can be obtained when the above three basic criteria are met. If an electrical circuit can be made by some electrical elements or some electrochemical elements making the impedance spectroscopy of this electrical circuit the same as the tested EIS of the electrode system, this electrical circuit is called equivalent circuit and the components included in the equivalent circuit are called equivalent elements.

The resistor $R$, inductor $L$, and capacitor $C$ are three common equivalent elements in the equivalent circuit [62]. It should be noticed that the impedance of the resistor is independent of frequency. There is only a real impedance component and no imaginary component $\left(Z=R, Z^{\prime}=R, Z^{\prime \prime}=0\right)$, whereas the inductor only has an imaginary component and the impedance of the inductor increases with the frequency, which results in a phase shift of 90 degrees of the current with respect to the voltage $\left(Z=i \omega L, Z^{\prime}=0\right.$, $\left.Z^{\prime \prime}=\omega L\right)$. The capacitor also only has an imaginary impedance component and the phase shift of 90 degree occurs between the current and the voltage. But opposite to the behavior of the inductor, the impedance of the capacitor decreases when the frequency increases $\left(Z=1 /(i \omega C), Z^{\prime}=0, Z^{\prime \prime}=1 /(\omega C)\right)$. 
The constant phase element (CPE) may also be introduced into the circuit as the substitute of the capacitor in some cases when the surface of the sample is rough, and the capacitor cannot represent the capacity of the equivalent electrical element ideally. The impedance of a CPE can be expressed as $Z_{C P E}=1 /\left((j \omega)^{\alpha} Y_{0}\right)$, where $Y_{0}$ has the unit of $\mathrm{s}^{\alpha} / \Omega$ and $\alpha$ is an exponent that varies in a range from 0.5 to 1 . The CPE can also be written simply as Q. When $\alpha$ equals 1, the constant phase $Q$ is identical to the capacitor and the unit of $Y_{0}$ becomes the same unit as farad (F). When $\alpha$ equals 0.5 , a 45-degree line can be seen from the complex plane graph.

The real electrochemical cell can be represented by a network of resistors, capacitors and sometimes inductors, which is known as the equivalent circuit. Figure 2-8 (a) shows the equivalent circuit of a purely capacitive coating. The resistor $R$ and the capacitor $C$ are in series in the model. When setting the frequency range to be $0.1 \mathrm{~Hz}$ to $1 \mathrm{MHz}$ and assigning the value of resistor to be $500 \Omega$ and the value of the capacitor to be $200 \mathrm{pF}$, the Nyquist plot displayed on the complex plane of the model is shown in Figure 2-8 (c). The X-axis represents the $Z_{\text {real }}$ and the $\mathrm{Y}$-axis represents the $Z_{\text {imagine. }}$. The $Z_{\text {total }}$ equals to the sum of $Z_{\text {real }}$ and $Z_{\text {imagine }}$ in the complex quantity.

The solution resistance $R_{s}$, the double layer capacitor $C_{d l}$ and the charge transfer resistance $R_{c t}$ (or polarization resistance $R_{p}$ ) form the simplified Randles model — one of the most popular models, as shown in Figure 2-8 (b). This simplified Randles model is usually used as a starting point for other more complex models [62]. If the polarization resistance $R_{p}$ is 
assigned to be $250 \Omega$ and the capacitance of $40 \mu \mathrm{F}$ and the solution resistance of $20 \Omega$ are assumed, the Nyquist plot can be obtained, as presented in Figure 2-8 (d).

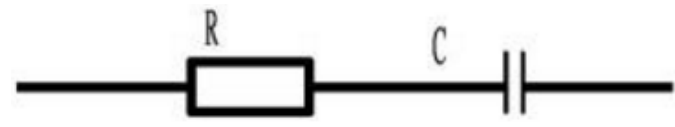

(a)

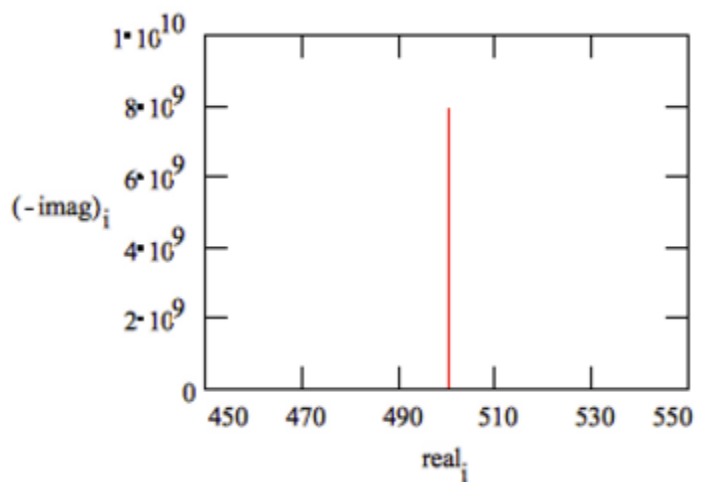

(c)

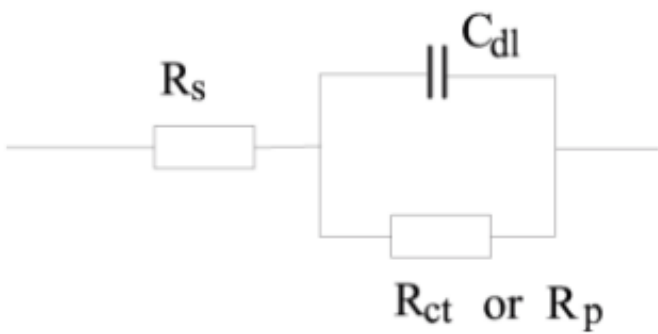

(b)

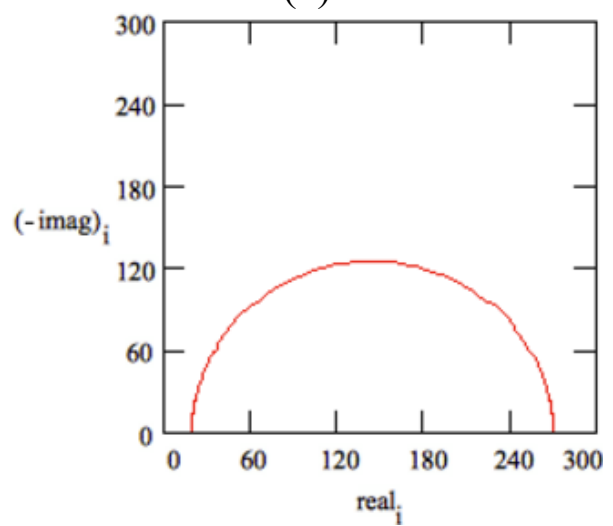

(d)

Figure 2-8 Equivalent circuits and corresponding Nyquist plots: (a) purely capacitive coating circuit; (b) simplified Randles circuit; (c) Nyquist plot of purely capacitive coating; (d) Nyquist plot of simplified Randles circuit [62]

Figure 2-9 (a) and Figure 2-9 (b) characterize the two circuits used for the equivalent circuits of a passive metal surface [69]. These two circuits were made base on the simplified Randles circuit, and they were further used to investigate the electrochemical behavior of titanium in an aqueous environment [70]. In Figure 2-9 (a), the $R$ and $Q$ elements in parallel are introduced in the simplified Randles circuit, which leads to an equivalent model of $R(Q(R(Q R)))$, as shown in Figure 2-9 (c). This model may be used to characterize the point defect in a metal [62]. 


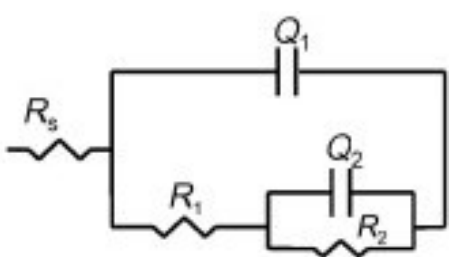

(a) Electrolyte

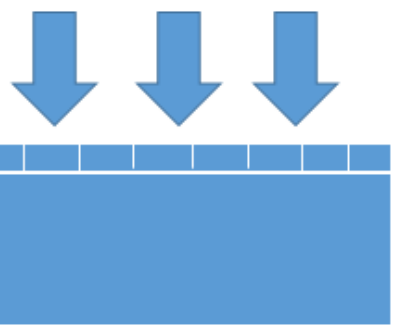

(c)

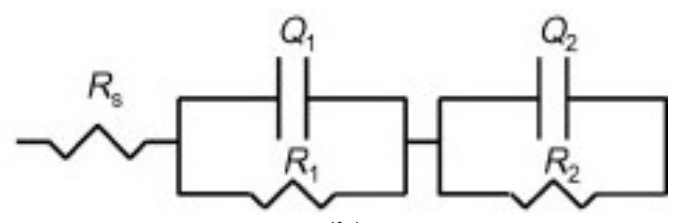

(b)

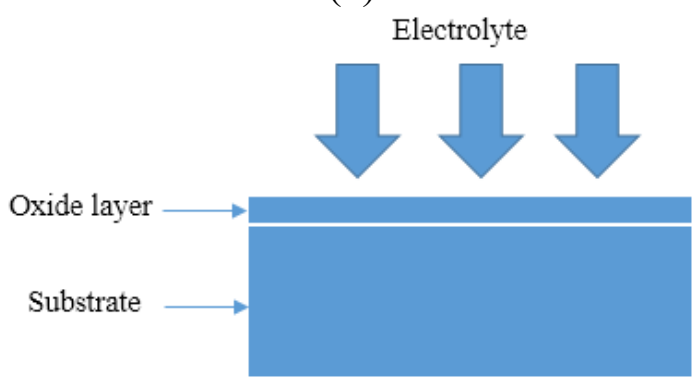

(d)

Figure 2-9 Interpretation of the equivalent circuits of a passive metal surface: (a) circuit 1 of $R(Q(R(Q R)))$; (b) circuit 2 of $R(Q R)(Q R)$; (c) illustration of the model of $R(Q(R(Q R)))$; (d) illustration of the model of $R(Q R)(Q R)$ [69]

However, in Figure 2-9 (b), the simplified Randles circuit uses two $(R Q)$ elements in series, which leads to an equivalent model of $R(Q R)(Q R)$. It is assumed that the passive film is more homogeneous than that of the model of $R(Q(R(Q R)))$, as shown in Figure 2-9 (d). It is suggested that model 2 is more appropriate to simulate highly protective oxide films and model 1 is more suitable for defective films [70]. Although these equivalent models have been used well, they are not unique. Alternative modes may also work for the equivalent circuits of a passive metal surface.

\subsubsection{Corrosion studies of metals using electrochemical methods}

Various electrochemical methods for corrosion studies are similar. Potentiodynamic polarization, cyclic polarization, potentiostatic polarization and EIS are commonly used for investigating the corrosion behavior of metals $[23,66]$. 
In the research of Popova et al., the corrosion of mild steels in acid media was studied using EIS, polarization resistance and dynamic polarization methods [71]. The frequency was set varying from $0.1 \mathrm{~Hz}$ to $95 \mathrm{kHz}$, and the $\mathrm{AC}$ amplitude was set to be $5 \mathrm{mV}$ in the EIS test. The setting $\pm 10 \mathrm{mV}$ vs. $E_{c o r r}$, at a scan rate of $0.1 \mathrm{mV} / \mathrm{s}$ was used for the polarization resistance test. As for the dynamic polarization test, the potential range was set to be $-200 \mathrm{mV}$ to $+120 \mathrm{mV}$ vs. $E_{\text {corr }}$ with a scan rate of $0.1 \mathrm{mV} / \mathrm{s}$.

Qiu et al. used the electrochemical corrosion test in the investigation of the corrosion behavior and surface change of a $\mathrm{Co}-\mathrm{Cr}$ and two $\mathrm{Ni}-\mathrm{Cr}$ dental alloys before and after simulated porcelain firing [72]. The potentiodynamic test was performed after each specimen reached a steady OCP for $2 \mathrm{~h}$, the scanning range $(-400 \mathrm{mV}$ to $+160 \mathrm{mV}$ vs. reference) with a scan rate of $1 \mathrm{mV} / \mathrm{s}$ was chosen.

The electrochemical method was also employed in the research of the effect of the passivity on the electrochemical corrosion behavior of 20SiMn low alloy steel and 0Cr13Ni5Mo and CrMnN stainless steels by Zheng et al. [73]. They set the start point of potentiodynamic polarization test to be $-250 \mathrm{mV}$ relative to corrosion potential with a sweep rate of $1 \mathrm{mV} / \mathrm{s}$, but they did not set a specific value of potential as the end point. They made the scan stop when the current reached a value of $10 \mathrm{~mA}$.

Kocijan et al. used the electrochemical methods of potentiodynamic measurement and EIS simultaneously when investigating the corrosion behavior of austenitic and duplex stainless steels in artificial saliva, and with the addition of fluoride [69]. They chose to start the 
potentiodynamic scan at $-250 \mathrm{mV}$ vs. reference with a scan rate of $1 \mathrm{mV} / \mathrm{s}$ and stop the scan when the scan reached the transpassive region. As for the EIS test, the frequency range was chosen from $65 \mathrm{kHz}$ to $5 \mathrm{MHz}$, with the $\mathrm{AC}$ amplitude of $10 \mathrm{mV}$.

Faget studied the effect of silicon on the electrochemical corrosion resistance of carbon steels in concentrated hydrochloric acid and carbonated water using potentiodynamic polarization [74]. A comparison of corrosion rate was made between the potentiodynamic polarization results of three carbon steels with different levels of silicon: $0.02,0,19$, and $0.94 \mathrm{wt} \%$.

Rodič and Milošev investigated the corrosion inhibition of pure aluminum and alloys AA2024-T3 and AA7075-T6 in cerium (III) and cerium (IV) salts [75]. The potentiodynamic polarization was performed with the start point of $-250 \mathrm{mV}$ vs. OCP to the anodic direction until the breakaway potential $E_{b}$ was reached, and the scan rate was set to be $1 \mathrm{mV} / \mathrm{s}$.

Roach et al. used the cyclic polarization test to evaluate the corrosion behavior of six nickel-chromium alloys in an artificial saliva solution [76]. The cyclic polarization test started from $-100 \mathrm{mV}$ vs. SCE with a scan rate of $0.1667 \mathrm{mV} / \mathrm{s}$ and the scan reversed back to the corrosion potential $E_{c o r r}$ of the alloys when the scan reached a current density of $1 \times 10^{-3} \mathrm{~A} / \mathrm{cm}^{2}$

Blin et al. used the cyclic polarization measurement and the EIS measurement together in 
the research to study the corrosion inhibition mechanism of new rare earth cinnamate compounds [77]. The cyclic polarization mainly focused on the primary corrosion inhibition mechanism and the pitting behavior. The cyclic potentiodynamic scan started from $-0.6 \mathrm{~V}$ vs. OCP to $+0.6 \mathrm{~V}$ vs. OCP and then reversed at a scan rate of $0.1667 \mathrm{mV} / \mathrm{s}$. In the meanwhile, EIS was used to determine how many fundamental parameters were associated with the electrochemical mechanism and to study the corrosion behavior of the materials. The frequency setting of EIS was from $0.01 \mathrm{~Hz}$ to $10 \mathrm{kHz}$ with an $\mathrm{AC}$ amplitude of $3 \mathrm{mV}$.

In the research of $\mathrm{Ni0.5Zn0.5Fe} 2 \mathrm{O} 4$ as a potential corrosion inhibitor for API 5L X80 steel in the acidic environment, Chaudhry et al. used EIS and potentiodynamic polarization methods to make the electrochemical measurements for analyzing the corrosion behavior of the steel [78]. The frequency of $0.01 \mathrm{~Hz}$ to $10 \mathrm{kHz}$ was applied with the $\mathrm{AC}$ amplitude of $10 \mathrm{mV}$ in the EIS tests. The range of potentiodynamic test was set to be $-250 \mathrm{mV}$ vs. OCP to $+750 \mathrm{mV}$ vs. OCP at a scan rate of $0.1667 \mathrm{mV} / \mathrm{s}$.

Although the reported corrosion researches were made on different materials in different solutions, the used electrochemical methods were similar. Potentiodynamic polarization, cyclic polarization, potentiostatic polarization and EIS methods may be used separately or be combined together according to the purpose of specific research. Even for the same method, the experimental setup may vary. Which electrochemical method will be chosen for a specific study depends on the purpose of the research and the instruments available in the lab. 


\section{Chapter 3: Electrochemical Corrosion Experiments}

\subsection{Specimen preparation}

\subsubsection{Chemical compositions and microstructure}

The specimens of Stellite 6 alloy and 17-4PH stainless steel used in this research are all cast products, provided by Kennametal Stellite Inc.. Table 3-1 [40] gives the chemical compositions (in weight) of Stellite 6 alloy and 17-4PH stainless steel. The hardness of Stellite 6 alloy and 17-4PH stainless steel were tested and the average values were HRC40 for the former and HRC34 for the latter.

Table 3-1 Chemical compositions of Stellite 6 alloy and 17-4PH stainless steel $(\mathrm{wt} \%)$ [40]

\begin{tabular}{|c|c|c|c|c|c|c|c|c|c|c|}
\hline & Co & Cr & Si & W & Cu & Mn & Ni & Mo & C & Fe \\
\hline Stellite 6 & Bal. & 28.87 & 1 & 4.31 & $/$ & 0.49 & 2.41 & $<1$ & 1.2 & 2.95 \\
\hline 17-4PH & $/$ & 15.8 & 0.44 & $/$ & 3.85 & 0.28 & 3.77 & 0.2 & $<0.1$ & Bal. \\
\hline
\end{tabular}

The microstructural analyses of the Stellite 6 alloy and 17-4PH stainless steel specimens were conducted on a Tescan Vega-II XMU SEM with backscatter electron imaging and an

Oxford EDX spectroscopy system, which is shown in Figure 3-1. The obtained images of the microstructures were captured at low magnification $(500 \mathrm{x})$ and high magnification $(2 \mathrm{kx})$. The EDX spectroscopy system was employed to provide the chemical constituents of the chosen area on the specimens. Hence, a local elemental content table of each specimen can be obtained. However, the EDX system is deficient to reflect the real alloy elements because of the inaccuracy results from the interfering of the carbon element. The 
carbon contents presented in the elemental content tables must be omitted, then the percentage of other alloy elements can be considered to be convincible.

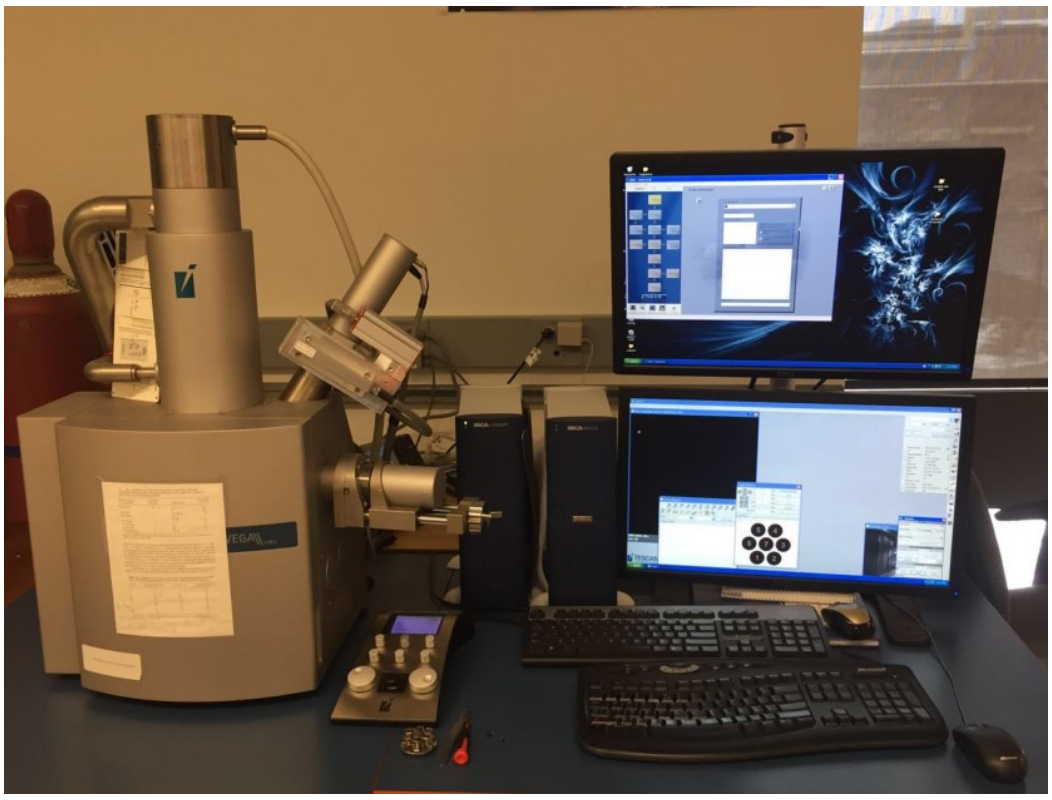

Figure 3-1 Tescan Vega-II XMU SEM with backscatter electron imaging and an EDX spectroscopy system

Figure 3-2 and Figure 3-3 show the SEM microstructures of Stellite 6 alloy and 17-4PH stainless steel at low magnification and high magnification respectively. It is observed that the microstructure of Stellite 6 alloy has three distinct phases in grey, black and white, respectively. The EDX results in Figure 3-4 to Figure 3-6 reveal that the white phase is W-rich carbide - $(\mathrm{W}, \mathrm{Co})_{6} \mathrm{C}[23]$, because it contains a very high $\mathrm{W}$ content, the black phase contains a very high $\mathrm{Cr}$ content so that it must be $\mathrm{Cr}$-rich carbide $-\mathrm{M}_{7} \mathrm{C}_{3}$, and the grey phase is Co solid solution, because it contains the highest Co content [23].

The microstructure of $17-4 \mathrm{PH}$ stainless steel exhibits a martensitic matrix. It is a martensitic precipitation hardened stainless steel. Figure 3-7 reports the EDX results of the martensitic matrix of 17-4PH stainless steel. 


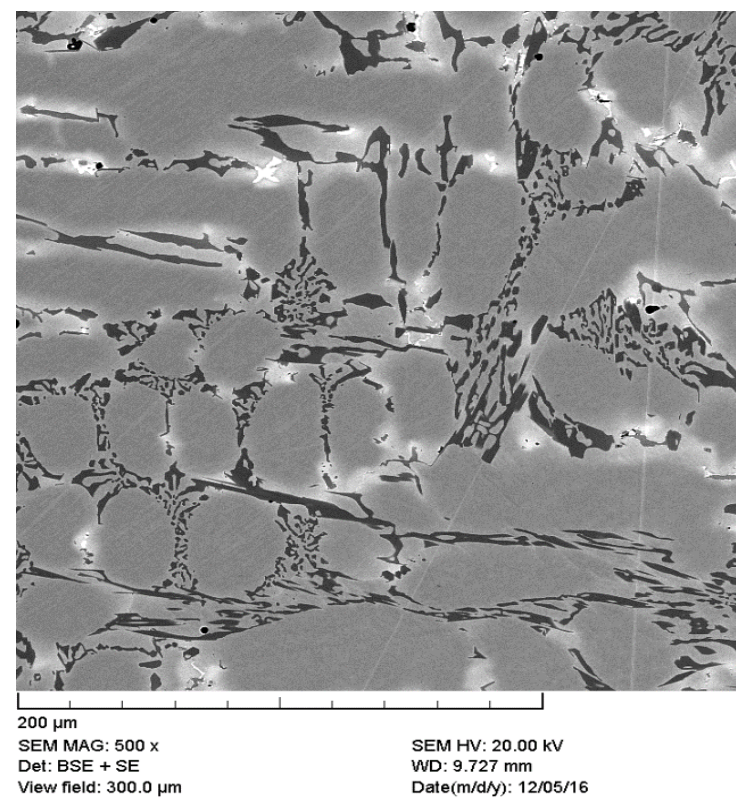

(a)

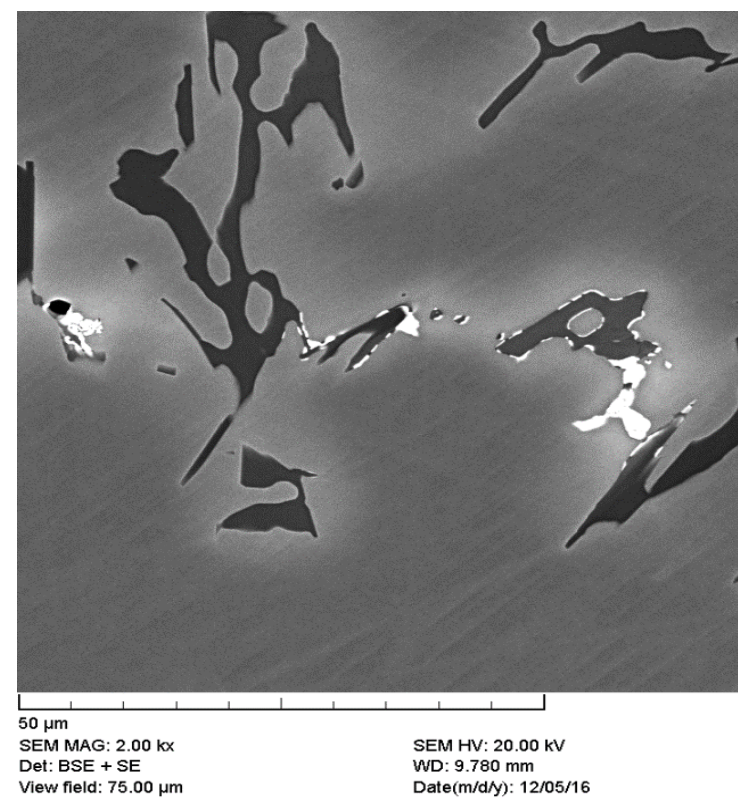

(b)

Figure 3-2 SEM microstructure of Stellite 6 alloy: (a) at low magnification;

(b) at high magnification

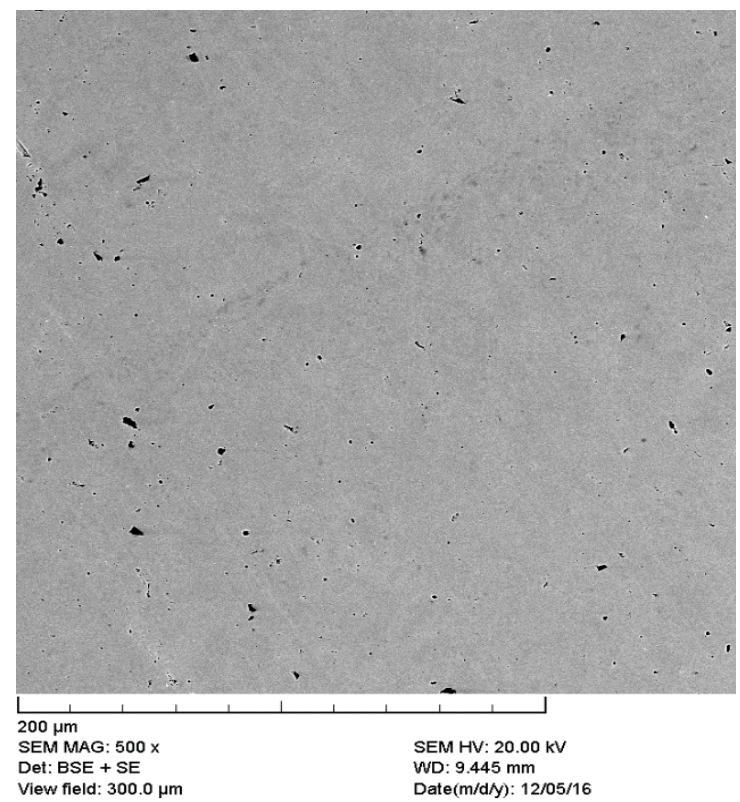

(a)

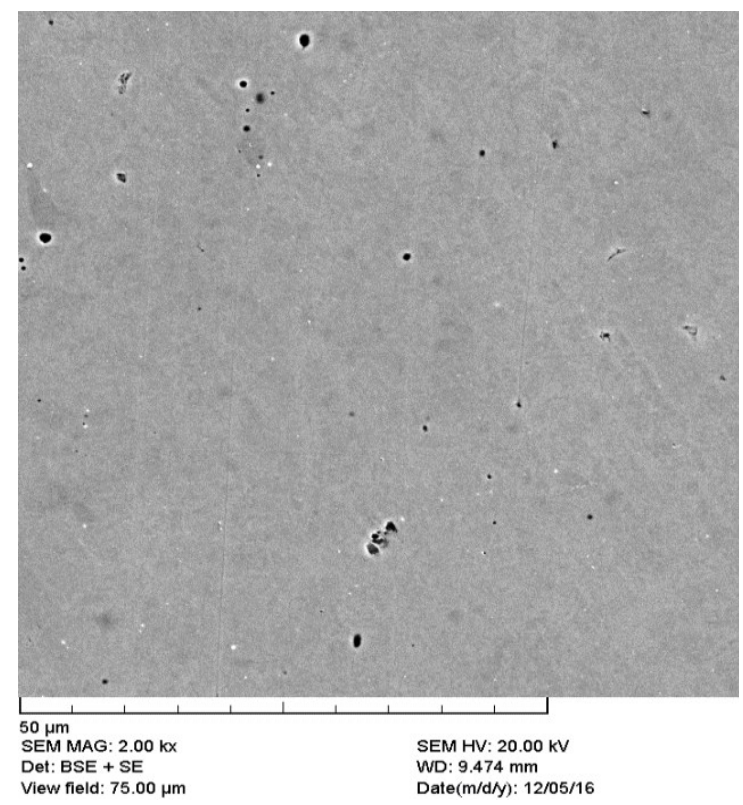

(b)

Figure 3-3 SEM microstructure of 17-4PH stainless steel: (a) at low magnification;

(b) at high magnification 


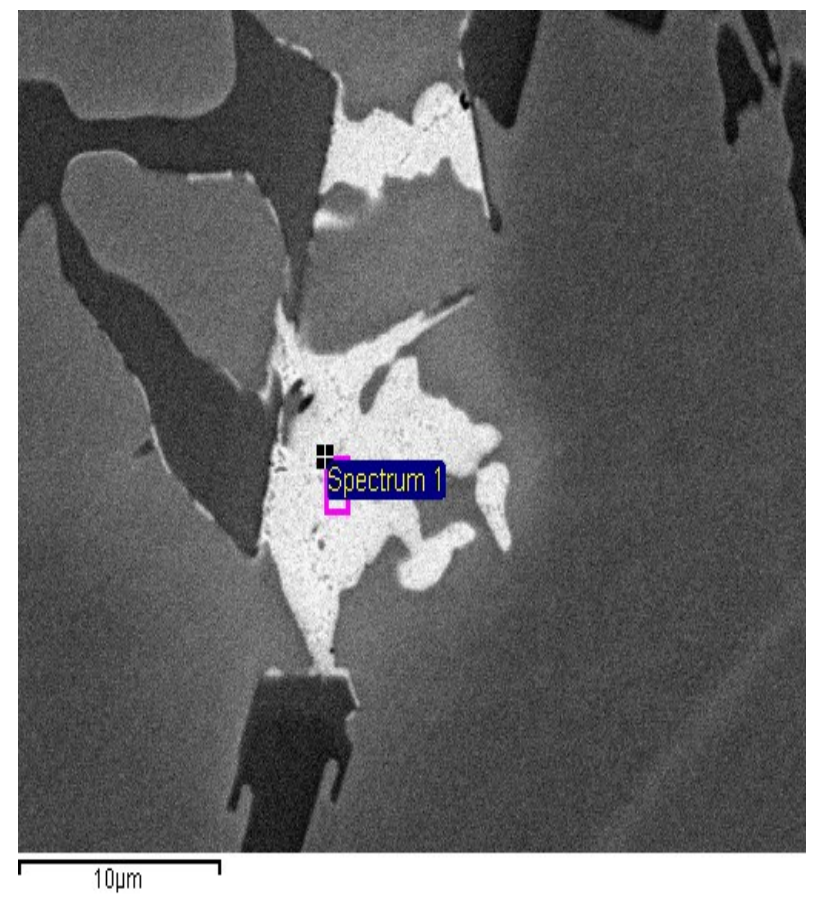

(a)

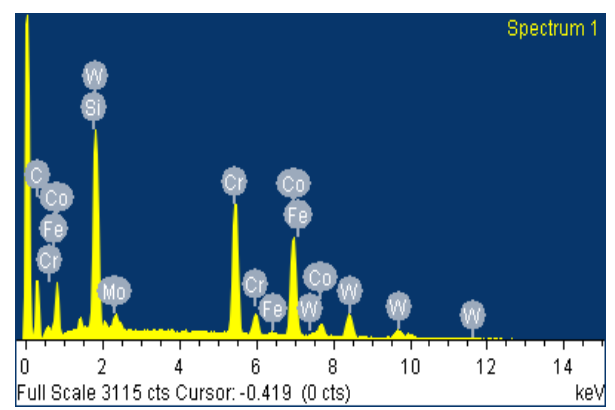

(b)

\begin{tabular}{|c|c|c|}
\hline Element & wt\% & at\% \\
\hline Si K & 1.58 & 3.86 \\
\hline Cr K & 24.14 & 31.78 \\
\hline Fe K & 0.69 & 0.85 \\
\hline Co K & 30.20 & 35.07 \\
\hline Mo L & 3.43 & 2.45 \\
\hline W M & 37.86 & 14.09 \\
\hline C & 2.09 & 11.90 \\
\hline Totals & 100.00 & 100.00 \\
\hline
\end{tabular}

(c)

Figure 3-4 EDX results of the white phase in Stellite 6 alloy: (a) SEM morphology;

(b) EDX spectrum; (c) elemental content table

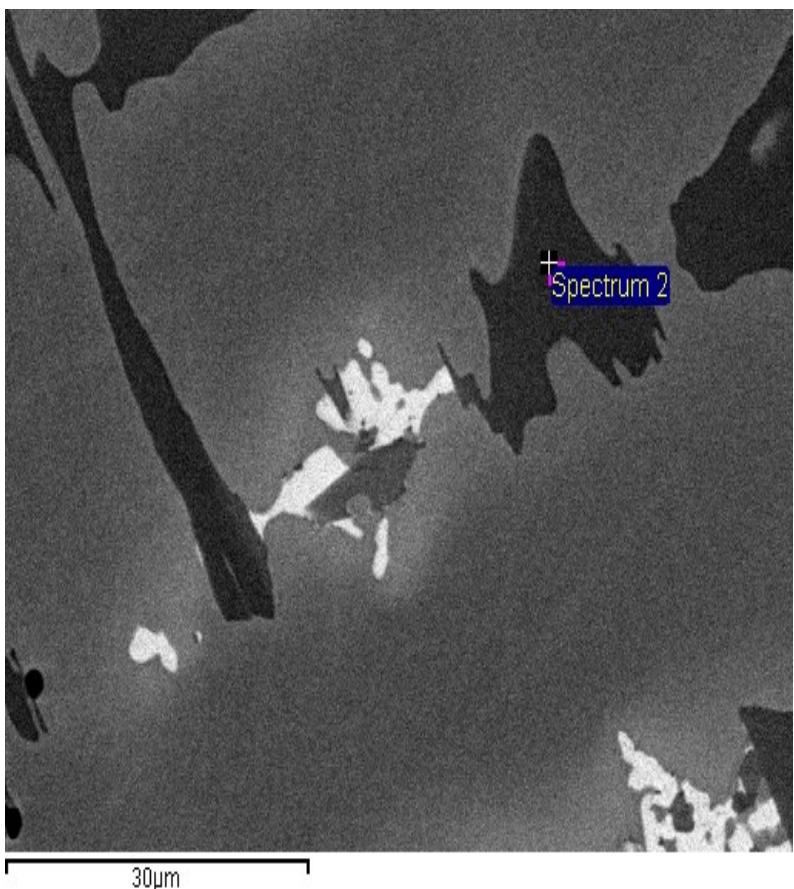

(a)

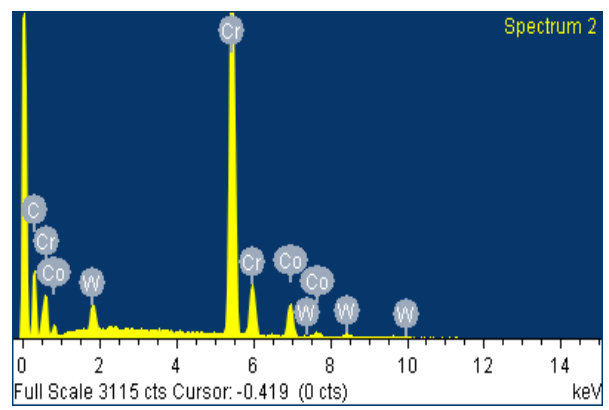

(b)

\begin{tabular}{|c|c|c|}
\hline Element & $\mathbf{w t} \%$ & $\mathbf{a t} \%$ \\
\hline Cr K & 73.49 & 57.91 \\
\hline Co K & 11.39 & 7.92 \\
\hline W M & 5.45 & 1.22 \\
\hline C & 9.66 & 32.96 \\
\hline Totals & 100.00 & 100.00 \\
\hline
\end{tabular}

(c)

Figure 3-5 EDX results of the black phase in Stellite 6 alloy: (a) SEM morphology; (b) EDX spectrum; (c) elemental content table 


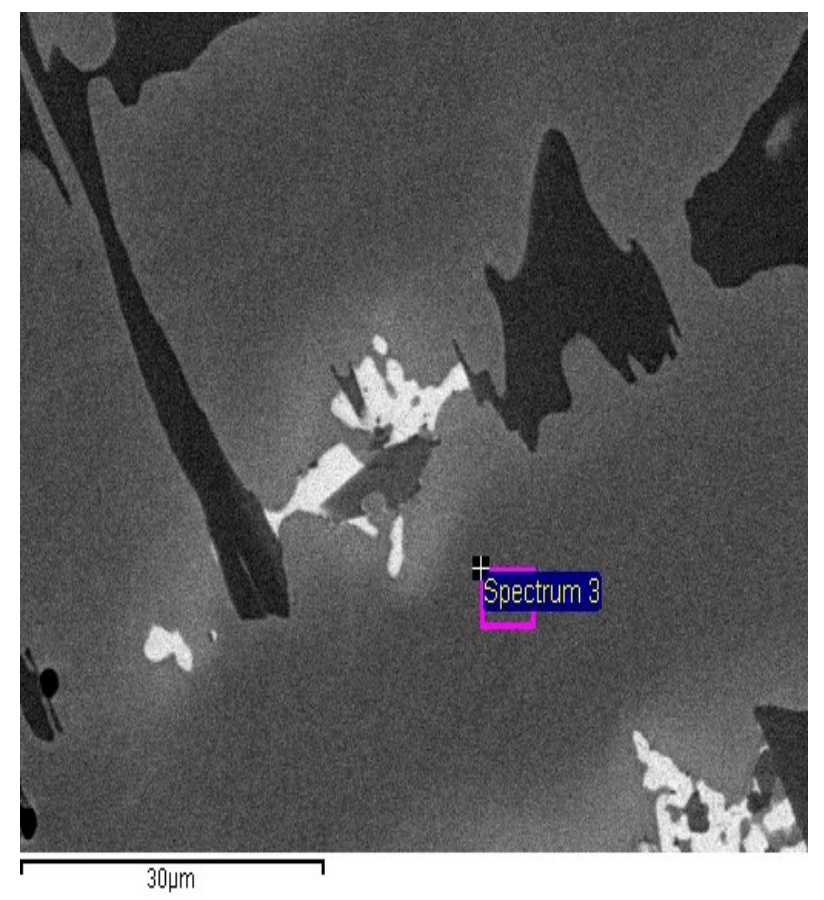

(a)

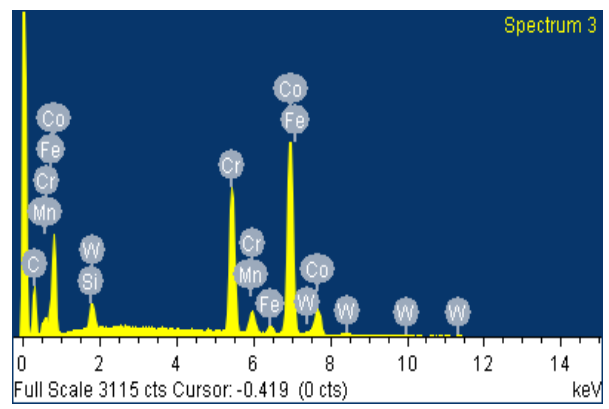

(b)

\begin{tabular}{|c|c|c|}
\hline Element & wt\% & at\% \\
\hline Si K & 1.08 & 1.92 \\
\hline Cr K & 24.85 & 23.99 \\
\hline Mn K & 0.88 & 0.80 \\
\hline Fe K & 1.78 & 1.60 \\
\hline Co K & 63.05 & 53.72 \\
\hline W M & 4.35 & 1.19 \\
\hline C & 4.01 & 16.77 \\
\hline Totals & 100.00 & 100.00 \\
\hline
\end{tabular}

(c)

Figure 3-6 EDX results of the grey phase in Stellite 6 alloy: (a) SEM morphology;

(b) EDX spectrum; (c) elemental content table

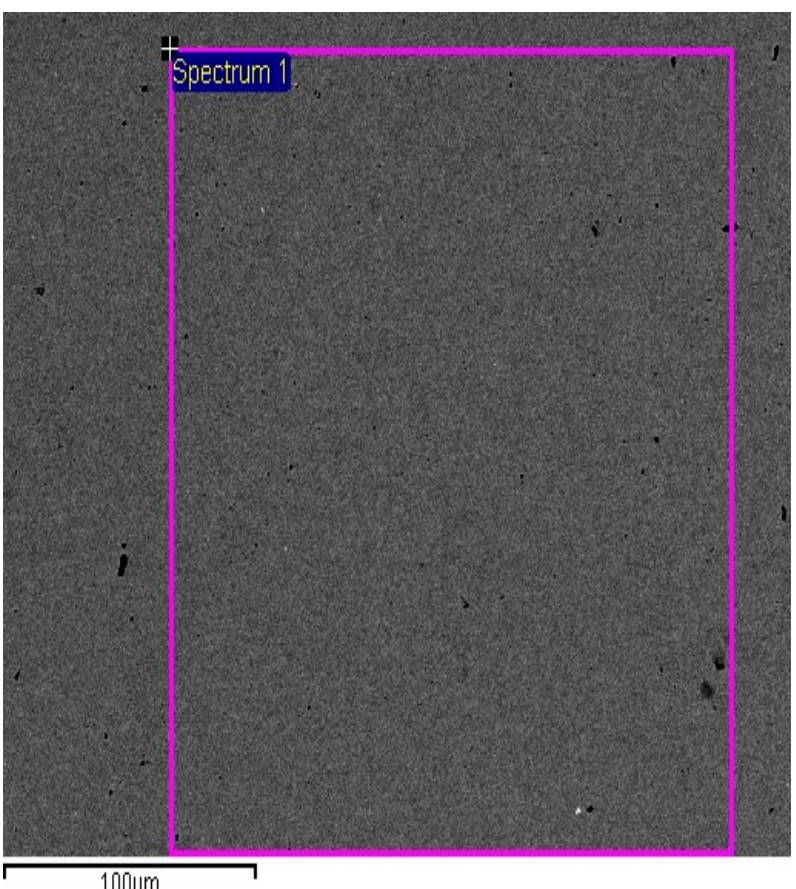

(a)

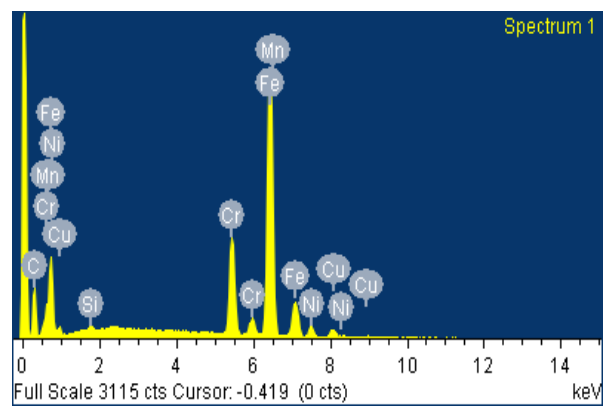

(b)

\begin{tabular}{|c|c|c|}
\hline Element & wt\% & at\% \\
\hline Si K & 0.39 & 0.68 \\
\hline Cr K & 15.71 & 14.88 \\
\hline Mn K & 1.21 & 1.09 \\
\hline Fe K & 71.29 & 62.89 \\
\hline Ni K & 4.03 & 3.38 \\
\hline Cu K & 3.96 & 3.07 \\
\hline C & 3.42 & 14.01 \\
\hline Totals & 100.00 & 100.00 \\
\hline
\end{tabular}

(c)

Figure 3-7 EDX results of the martensitic matrix of 17-4PH stainless steel: (a) SEM morphology; (b) EDX spectrum; (c) elemental content table 


\subsubsection{Surface preparation}

The Stellite 6 alloy and 17-4PH stainless steel specimens were around plates with the diameter of $16 \mathrm{~mm}$ and the thickness of $1.6 \mathrm{~mm}$. To meet the requirements of electrochemical tests, the specimen surface was polished according to the ASTM G59 Standard [79]. The polishing was conducted on a Buehler MetaServ 250 single polisher, shown in Figure 3-8, following the wet polishing procedures, which can achieve a mirrorlike surface [80]. SiC abrasive papers were used from 240 grit to 600 grit in a sequence. The final polishing process was made right before the electrochemical test, which used $3 \mu \mathrm{m}$ suspension and polishing cloth to minimize the effects of preformed oxide films on the specimen surface because oxide films immediately formed on the specimen surface once the grinding process was finished.

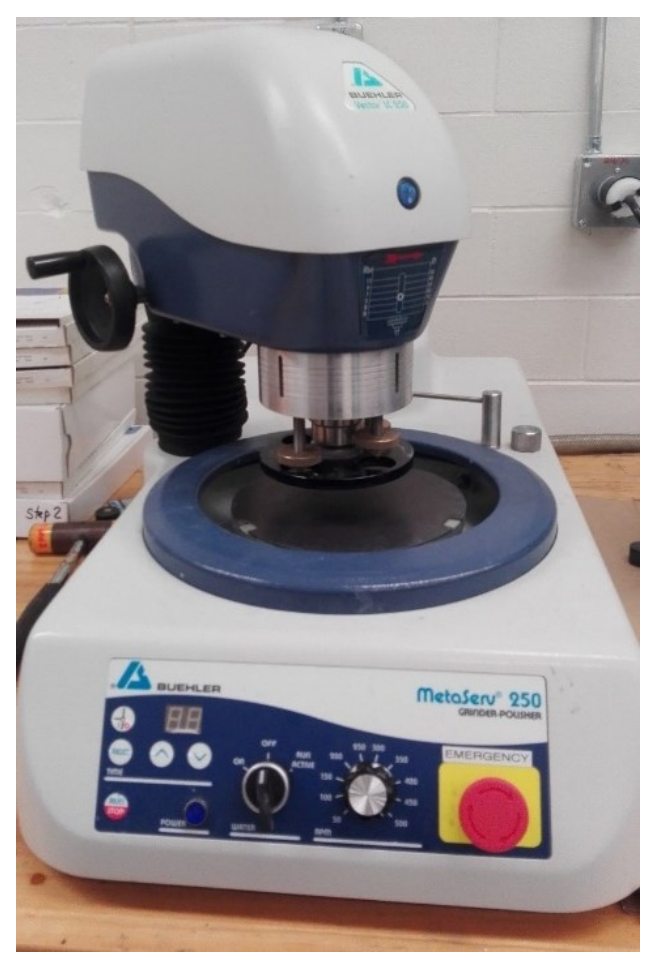

Figure 3-8 MetaServ 250 single polisher 
Degreasing with acetone and rinsing with deionized water were the final procedures of the specimen surface polishing, which were made within $30 \mathrm{~min}$ before the electrochemical test was started [79].

\subsection{Test solution preparation}

Before making the solutions of morpholine and cyclohexylamine, two vital factors must be aware with neutralizing amines: (1) Basicity; (2) Distribution ratio [81].

Basicity refers to the ability of the amine to boost the $\mathrm{pH}$ of the neutralized water, and the general reaction of amines with water is expressed by Equation 3-1:

$$
\mathrm{RNH}_{2}+\mathrm{H}_{2} \mathrm{O} \rightarrow \mathrm{RNH}_{3}^{+}+\mathrm{OH}^{-}
$$

The base hydrolysis constant $K_{b}$ for amines is defined by Equation 3-2. An amine with a smaller value of $K_{b}$ will raise the $\mathrm{pH}$ of pure water less than an amine with a larger value of $K_{b}$ :

$$
K_{b}=\left[\mathrm{RNH}_{3}^{+}\right]\left[\mathrm{OH}^{-}\right] /\left[\mathrm{RNH}_{2}\right]
$$

Because neutralizing amines are volatile and they could follow the steam into the feed water system, the distribution ratio (DR) is used to measure the volatility of the amine, as given by Equation 3-3:

$$
D R=[\text { amine in steam }] /[\text { amine in the water }]
$$


Morpholine is an organic chemical compound and a colorless liquid having the chemical formula $\mathrm{O}\left(\mathrm{CH}_{2} \mathrm{CH}_{2}\right)_{2} \mathrm{NH}$ (Figure 3-9) with the molecular weight of $87.12 \mathrm{~g} / \mathrm{mol}$ [82]. It has the features of both amine and ether functional groups because of the heterocycle, thus it only has one NH linkage. The density of morpholine is $1.007 \mathrm{~g} / \mathrm{cm}^{3}$. It is soluble in water and $K_{b}$ of morpholine is $3.1 \times 10^{-6}$ [83]. The DR of morpholine is 0.4 [84].

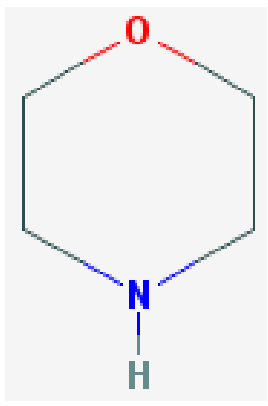

Figure 3-9 Structure of morpholine [82]

Cyclohexylamine is also an organic compound, belonging to the aliphatic amine class. It is a colorless liquid having the chemical formula $\mathrm{C}_{6} \mathrm{H}_{11} \mathrm{NH}_{2}$ (Figure 3-10) with the molecular weight of $99.18 \mathrm{~g} / \mathrm{mol}$ [85]. It has two NH linkages. The density of cyclohexylamine is $0.867 \mathrm{~g} / \mathrm{cm}^{3}$. It is soluble in water and $K_{b}$ of cyclohexylamine is $440 \times$ $10^{-6}[83]$. The DR of cyclohexylamine is 4.7 [84].

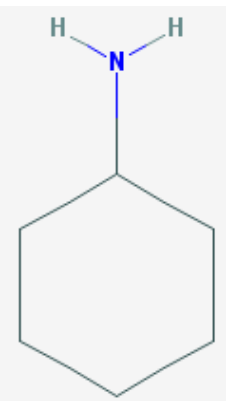

Figure 3-10 Structure of cyclohexylamine [83] 
For sodium hydroxide, Equation 3-1 and Equation 3-2 can be written as Equation 3-4 and Equation 3-5:

$$
\begin{gathered}
\mathrm{NaOH} \rightarrow \mathrm{Na}^{+}+\mathrm{OH}^{-} \\
K_{b}=\left[\mathrm{Na}^{+}\right]\left[\mathrm{OH}^{-}\right] /[\mathrm{NaOH}]
\end{gathered}
$$

Different from morpholine and cyclohexylamine, sodium hydroxide is an inorganic compound. It is a white solid having the chemical formula $\mathrm{NaOH}$ with the molecular weight of $40 \mathrm{~g} / \mathrm{mol}$ [86]. The density of sodium hydroxide is $2.13 \mathrm{~g} / \mathrm{cm}^{3}$. It is a highly caustic alkali and is highly soluble in water. The $K_{b}$ of sodium hydroxide is 8.51 [87]. There is no DR for sodium hydroxide because it is not volatile.

\subsubsection{Morpholine solution}

For each test in morpholine solution, $1.8 \mathrm{~L}$ higher concentration morpholine solution and 1 L lower concentration morpholine solution were prepared. The preparation procedures are as follows:

(1) Pour 1.8 L and $1 \mathrm{~L}$ deionized water into a $2 \mathrm{~L}$ flat bottom flask and a $1 \mathrm{~L}$ flat bottom flask respectively.

(2) Add $2 \mathrm{ml} \mathrm{99 \%} \mathrm{morpholine} \mathrm{into} \mathrm{the} 2 \mathrm{~L}$ flat bottom flask using a $5 \mathrm{ml}$ measuring cylinder and stir the solution until the morpholine is uniformly mixed (1122.2 ppm morpholine solution). 
(3) Add $160 \mu 1$ morpholine into the $1 \mathrm{~L}$ flat bottom flask using a $10 \mu \mathrm{l}$ high precision pipette and stir the solution to make the solution fully mixed (161.6 ppm morpholine solution).

(4) Rinse the bridge tube, the flat specimen holder, the two graphite rods (which were fitted into the threaded adapter of T24/40 joints) and the K0047 corrosion flask together with the $161.6 \mathrm{ppm}$ morpholine solution first. Fill the bridge tube in K0047 corrosion cell, shown in Figure 3-11, with 161.6 ppm morpholine solution.

(5) Pour the rest of the $161.6 \mathrm{ppm}$ morpholine solution (around $900 \mathrm{~mL}$ ) into the K0047 corrosion cell.

\subsubsection{Cyclohexylamine solution}

For each test in cyclohexylamine solution, $1.8 \mathrm{~L}$ higher concentration cyclohexylamine solution and $1 \mathrm{~L}$ lower concentration cyclohexylamine solution were prepared. The preparation procedures are as follows:

(1) Pour $1.8 \mathrm{~L}$ and $1 \mathrm{~L}$ deionized water into a $2 \mathrm{~L}$ flat bottom flask and a $1 \mathrm{~L}$ flat bottom flask respectively.

(2) Add $5 \mathrm{ml} \mathrm{99 \%} \mathrm{cyclohexylamine} \mathrm{into} \mathrm{the} 2 \mathrm{~L}$ flat bottom flask using a $5 \mathrm{ml}$ measuring cylinder and stir the solution until the cyclohexylamine is uniformly mixed (2162.8 ppm cyclohexylamine solution).

(3) Add $7 \mu \mathrm{l}$ cyclohexylamine into the $1 \mathrm{~L}$ flat bottom flask using a $10 \mu \mathrm{l}$ high precision pipette and stir the solution to make the solution fully mixed (6 ppm cyclohexylamine solution). 
(4) Rinse the bridge tube, the flat specimen holder, the two graphite rods (which were fitted into the threaded adapter of T24/40 joints) and the K0047 corrosion flask together with the $6 \mathrm{ppm}$ cyclohexylamine solution first. Fill the bridge tube in K0047 corrosion cell in Figure 3-11 with 6 ppm cyclohexylamine solution.

(5) Pour the rest of the $6 \mathrm{ppm}$ cyclohexylamine solution (around $900 \mathrm{~mL}$ ) into the K0047 corrosion cell.

\subsubsection{Sodium hydroxide solution}

For each test in sodium hydroxide solution, $1 \mathrm{~L}$ sodium hydroxide solution was prepared. The preparation procedures are shown below:

(1) Pour $1 \mathrm{~L}$ deionized water into a $1 \mathrm{~L}$ flat bottom flask.

(2) Weigh $0.1008 \mathrm{~g}$ of sodium hydroxide.

(3) Put the $0.1008 \mathrm{~g}$ of sodium hydroxide into the $1 \mathrm{~L}$ flat bottom flask and stir the flask until the sodium hydroxide is fully mixed with deionized water (primary solution).

(4) Measure $20 \mathrm{ml}$ the mixed primary solution using a $50 \mathrm{ml}$ measuring cylinder and pour the solution into another $1 \mathrm{~L}$ flat bottom flask.

(5) Dilute the primary solution with $980 \mathrm{ml}$ deionized water and stir the $1 \mathrm{~L}$ flat bottom flask until the solution is fully mixed ( $2 \mathrm{ppm}$ sodium hydroxide solution).

(6) Rinse the bridge tube, the flat specimen holder, the two graphite rods (which were fit into the threaded adapter of T24/40 joints) and the K0047 corrosion flask together with the 2 ppm sodium hydroxide solution first. Fill the bridge tube in K0047 corrosion cell, shown in Figure 3-11, with 2 ppm sodium hydroxide solution. 
(7) Pour the rest of the $2 \mathrm{ppm}$ sodium hydroxide solution (around $900 \mathrm{~mL}$ ) into the K0047 corrosion cell.

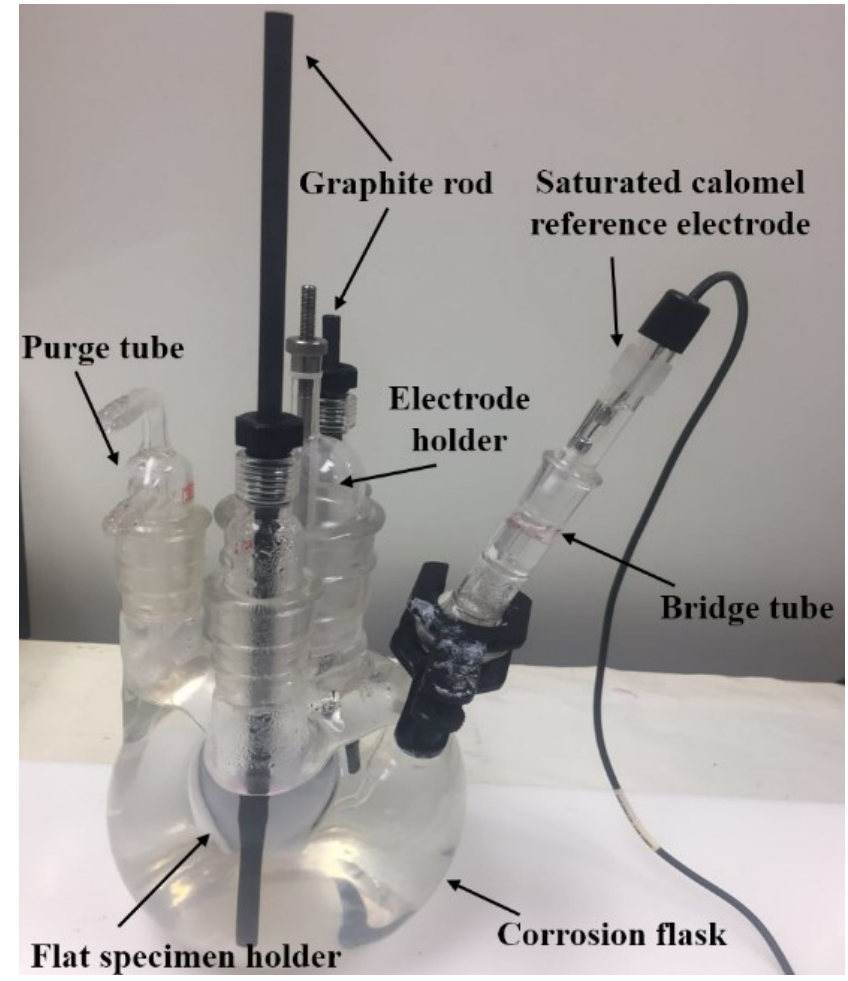

Figure 3-11 K0047 corrosion cell

\section{3 pH control for testing environment}

To simulate the real industrial amine environment, the $\mathrm{pH}$ level during the electrochemical corrosion tests must be maintained at a relatively steady state, and oxygen and carbon dioxide in the air must be kept out of the testing environment. Hence argon gas was used to make bubbles get rid of air. To measure the $\mathrm{pH}$, Oakton pHTestr $30 \mathrm{pH}$ Tester was used for all the tests. The $\mathrm{pH}$ tests verified that the minimal $\mathrm{pH}$ variations were quite acceptable for the later electrochemical experiments. The $\mathrm{pH}$ range was found to be 9.2 to 9.8 . 


\subsubsection{Morpholine solution}

The previous tests showed that the $\mathrm{pH}$ of the morpholine solution dropped quickly in the corrosion cell (Figure 3-11), which greatly influenced the EIS and polarization curves. The change in the $\mathrm{pH}$ made the test hard to investigate the real situation of the specimen when it became corroded. Therefore, an effective means to maintain the $\mathrm{pH}$ substantially constant in the corrosion cell was necessary.

First, a method of using the $161.6 \mathrm{ppm}$ morpholine solution with $\mathrm{pH}$ around 9.5 and purging argon gas into the corrosion cell directly was tried, but the $\mathrm{pH}$ decreased dramatically. Then the same concentration of $161.6 \mathrm{ppm}$ morpholine solution with $\mathrm{pH}$ around 9.5 in both the $2 \mathrm{~L}$ flat bottom flask and the corrosion cell was used. The tube for connecting the corrosion cell and the flask was used to make a $\mathrm{pH}$ compensation system, as demonstrated in Figure 3-12. Argon gas was purged into the system. However, the pH in the $2 \mathrm{~L}$ flat bottom flask and corrosion cell both decreased and the $\mathrm{pH}$ change in the corrosion cell was greater. 


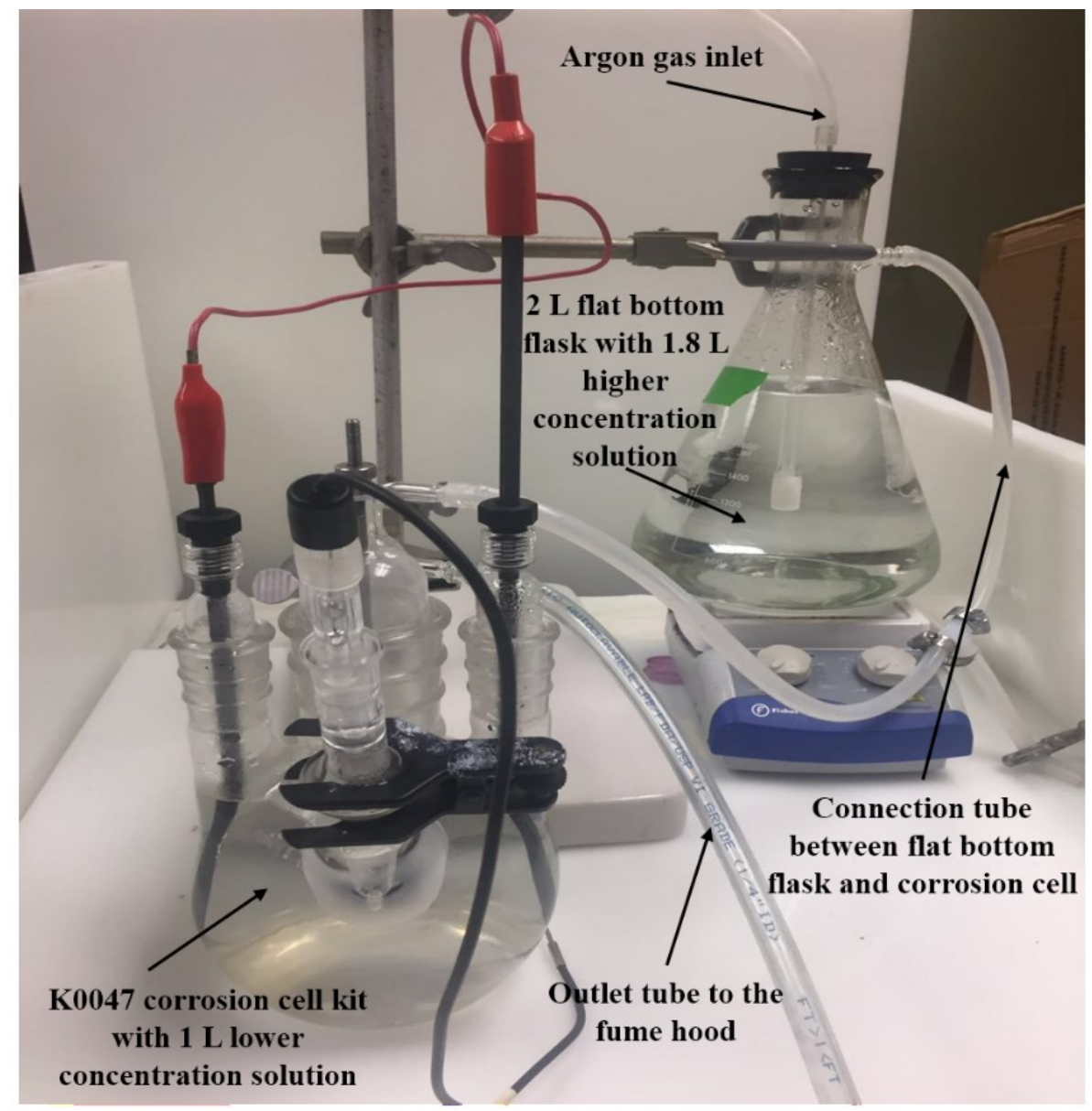

Figure 3-12 pH compensation system

According to the Raoult's law [88] that the partial vapor pressure of each component of an ideal mixture of liquids is equal to the vapor pressure of the pure component multiplied by its mole fraction in the mixture, that is, the transmission from gas to liquid and the transmission from liquid to gas is not $100 \%$. As a result, the morpholine transferred from the $2 \mathrm{~L}$ flat bottom flask to the corrosion cell was limited after experiencing the transmission.

After that, trials were made to make the $\mathrm{pH}$ of the morpholine solution in the corrosion cell around $9.5(161.6 \mathrm{ppm})$ and make the concentration of the morpholine solution in the $2 \mathrm{~L}$ 
flat bottom flask much higher. In this case, they could reach equilibrium to some extent to make the $\mathrm{pH}$ in the corrosion cell constant. In Figure 3-13, $2805.5 \mathrm{ppm}$ morpholine solution was made by adding $5 \mathrm{ml}$ morpholine and $1800 \mathrm{ml}$ deionized water into the $2 \mathrm{~L}$ flat bottom flask making the $\mathrm{pH}$ of the solution to be 10.36 . Adding $1000 \mathrm{ml}$ deionized water and $160 \mu 1$ morpholine into the corrosion cell made the $\mathrm{pH}$ of the corrosion cell to be 9.52 (161.6 ppm). When the system started to bubble, the $\mathrm{pH}$ in the small bubbler was measured every $30 \mathrm{~min}$. The curve for $5 \mathrm{ml}$ morpholine in Figure 3-13 shows the $\mathrm{pH}$ change in the corrosion cell within $2 \mathrm{~h}$, and the $\mathrm{pH}$ increased rapidly.

After several trials, the ratio of adding $2 \mathrm{ml} \mathrm{99 \%} \mathrm{morpholine} \mathrm{into} \mathrm{the} 2 \mathrm{~L}$ flat bottom flask and adding $160 \mu \mathrm{l}$ morpholine into the $1 \mathrm{~L}$ corrosion cell was found to be good for the $\mathrm{pH}$ constant after three repeated tests using $2 \mathrm{ml}$ morpholine in the $2 \mathrm{~L}$ flat bottom flask. The data were recorded in Table 3-2. These three tests, illustrated in Figure 3-13, show the same trend that the $\mathrm{pH}$ did not change much and they were all around $\mathrm{pH} 9.5$ within $2 \mathrm{~h}$. When accounting for the inaccuracy and experimental error of the $\mathrm{pH}$ tester, these $\mathrm{pH}$ changes can be negligible.

Table 3-2 The $\mathrm{pH}$ exploration data of morpholine solution for $2 \mathrm{~h}$

\begin{tabular}{|c|c|c|c|c|c|}
\hline Test duration (h) & $\mathbf{0}$ & $\mathbf{0 . 5}$ & $\mathbf{1}$ & $\mathbf{1 . 5}$ & $\mathbf{2}$ \\
\hline $\mathbf{5} \mathbf{~ m l}$ morpholine test $\mathbf{( p H )}$ & 9.52 & 9.50 & 9.87 & 9.93 & 9.95 \\
\hline $\mathbf{2} \mathbf{~ m l}$ morpholine test 1 (pH) & 9.66 & 9.55 & 9.54 & 9.59 & 9.58 \\
\hline $\mathbf{2} \mathbf{~ m l}$ morpholine test 2 (pH) & 9.59 & 9.55 & 9.54 & 9.54 & 9.54 \\
\hline $\mathbf{2} \mathbf{~ m l ~ m o r p h o l i n e ~ t e s t ~ 3 ~ ( p H ) ~}$ & 9.50 & 9.50 & 9.49 & 9.49 & 9.52 \\
\hline
\end{tabular}




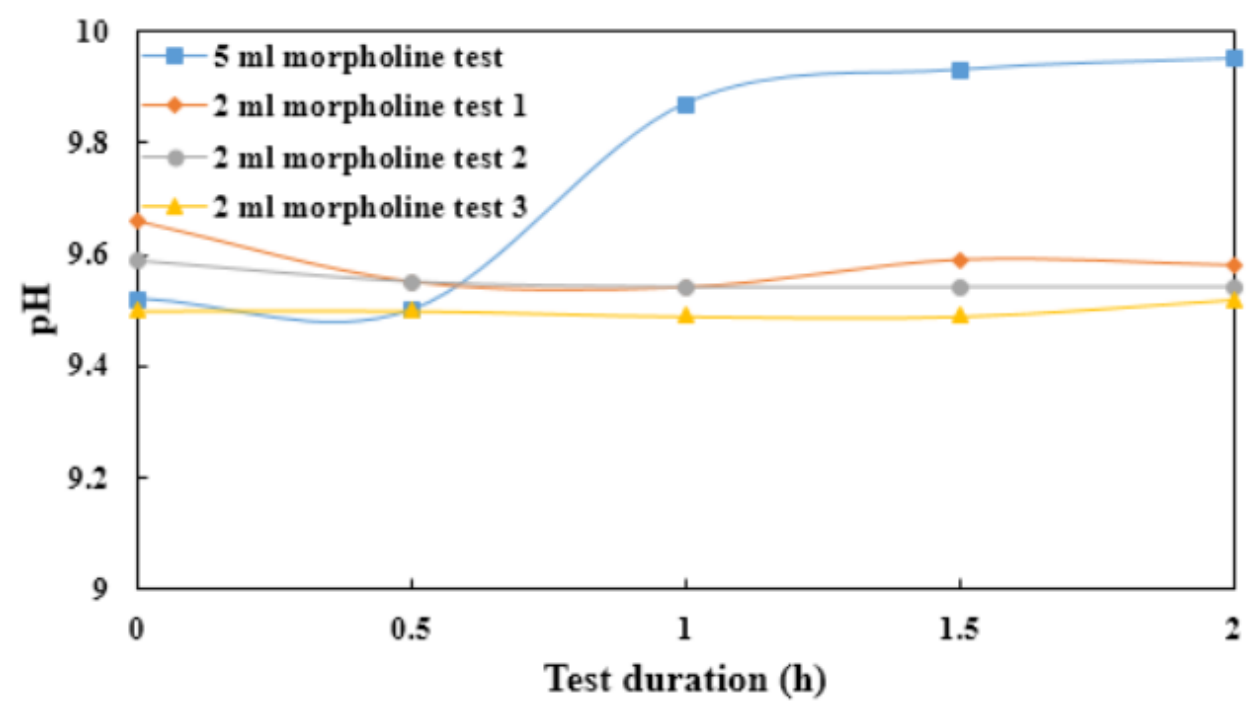

Figure 3-13 The $\mathrm{pH}$ exploration of morpholine solution for $2 \mathrm{~h}$

Since the $\mathrm{pH}$ did not change much within $2 \mathrm{~h}$, the test duration was extended to a longer time to test the time limit of the $\mathrm{pH}$ maintaining ability of the $\mathrm{pH}$ compensation system in morpholine solution. Table 3-3 and Figure 3-14 demonstrate the trend that the $\mathrm{pH}$ fluctuated around 9.5 for almost four days before the $\mathrm{pH}$ experienced a decline to 9.26. This $\mathrm{pH}$ variation was acceptable because it showed that the sufficient time could be provided for performing the following experiments, which was validated by later tests, as shown in Table 3-4. Figure 3-15 is a lucid visualization of this table.

Table 3-3 The $\mathrm{pH}$ exploration data of morpholine solution for $120 \mathrm{~h}$

\begin{tabular}{|c|c|c|c|c|c|c|c|c|}
\hline Time (h) & $\mathbf{0}$ & $\mathbf{0 . 3 3}$ & $\mathbf{0 . 6 7}$ & $\mathbf{1 . 1 7}$ & $\mathbf{1 . 6 7}$ & $\mathbf{2 . 6 7}$ & $\mathbf{3 . 6 7}$ & $\mathbf{4 . 6 7}$ \\
\hline $\mathbf{p H}$ & 9.65 & 9.60 & 9.62 & 9.64 & 9.65 & 9.68 & 9.67 & 9.68 \\
\hline Time (h) & $\mathbf{6 . 6 7}$ & $\mathbf{1 0 . 6 7}$ & $\mathbf{2 7}$ & $\mathbf{3 0}$ & $\mathbf{5 5}$ & $\mathbf{7 8}$ & $\mathbf{1 0 2}$ & $\mathbf{1 2 0}$ \\
\hline $\mathbf{p H}$ & 9.70 & 9.68 & 9.58 & 9.59 & 9.61 & 9.54 & 9.56 & 9.26 \\
\hline
\end{tabular}




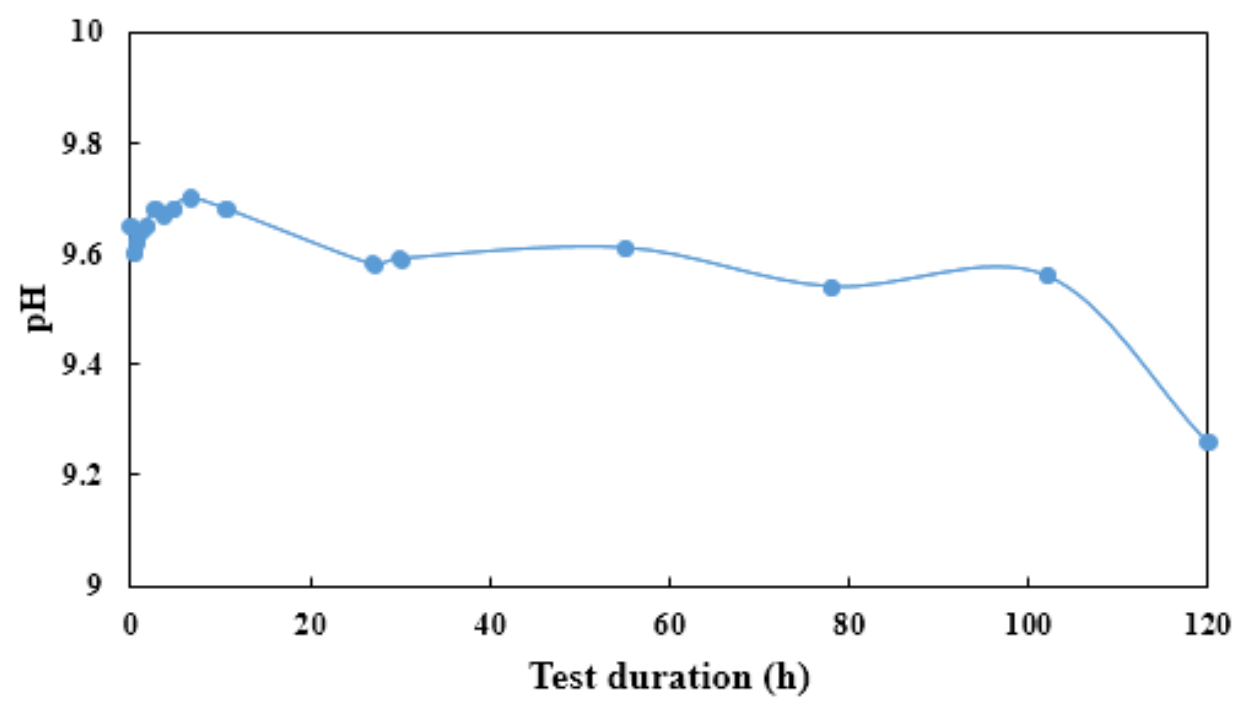

Figure 3-14 The pH exploration of morpholine solution for $120 \mathrm{~h}$

Table 3-4 The $\mathrm{pH}$ variation of morpholine solution

\begin{tabular}{|c|c|c|c|}
\hline & Initial pH & End pH & pH variation \\
\hline Test 1 (11 h) & 9.52 & 9.53 & 0.01 \\
\hline Test 2 (18 h) & 9.53 & 9.53 & 0 \\
\hline Test 3 (24 h) & 9.44 & 9.42 & -0.02 \\
\hline Test 4 (60 h) & 9.58 & 9.39 & -0.19 \\
\hline Test 5 (72 h) & 9.61 & 9.41 & -0.20 \\
\hline Test 6 (120 h) & 9.53 & 9.35 & -0.18 \\
\hline
\end{tabular}

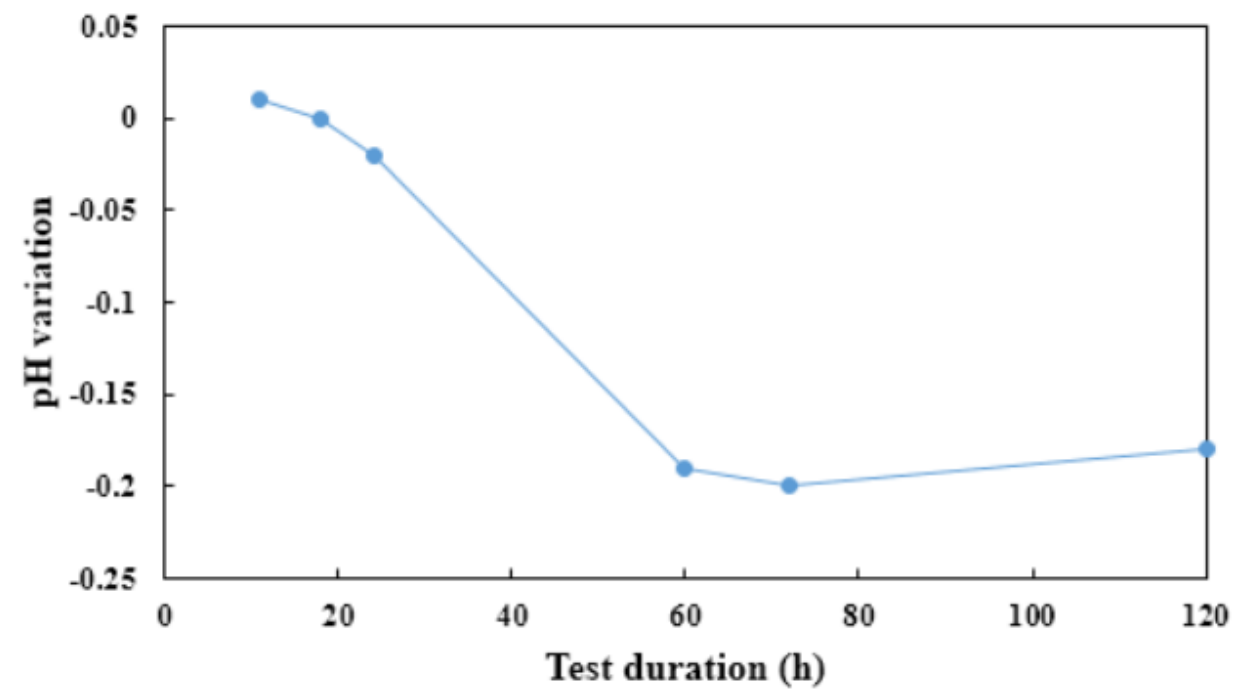

Figure 3-15 The $\mathrm{pH}$ variation of morpholine solution with time 
In summary, by setting a high concentration solution of morpholine in the $2 \mathrm{~L}$ flat bottom flask and a lower concentration solution $(\mathrm{pH} \approx 9.5)$ of morpholine in the corrosion cell, the $\mathrm{pH}$ in the corrosion cell can be assumed constant according to the resolution of the $\mathrm{pH}$ tester. Although the concentrations of the morpholine solutions were prepared as the same, the initial $\mathrm{pH}$ of the corrosion cell varied in a range from 9.39 to 9.74 .

\subsubsection{Cyclohexylamine solution}

Tests in morpholine solution show that the $\mathrm{pH}$ of lower concentration solution in the corrosion cell can be compensated by higher concentration solution in the prior flask over a certain period. An article reported that the cyclohexylamine has a high DR of 4.7, whereas this value of morpholine is only 0.4 [84]. This means that morpholine tends to stay in the liquid phase, and cyclohexylamine is easy to become vapor. To create a compensation system used for cyclohexylamine solution, a higher concentration of cyclohexylamine solution was required in the $2 \mathrm{~L}$ flat bottom flask. Thus, the tests in cyclohexylamine solution started with $6 \mathrm{ppm}$ cyclohexylamine solution in the corrosion cell and $2162.8 \mathrm{ppm}$ cyclohexylamine solution in the $2 \mathrm{~L}$ flat bottom flask.

The $\mathrm{pH}$ of cyclohexylamine solution in the corrosion cell was monitored over $48 \mathrm{~h}$, and the pH data are presented in Table 3-5 and are also illustrated in Figure 3-16. It can be seen that the $\mathrm{pH}$ remains stable for the first $24 \mathrm{~h}$, after that the $\mathrm{pH}$ experienced a slightly declining trend from $24 \mathrm{~h}$ to $48 \mathrm{~h}$. However, the $\mathrm{pH}$ did not change significantly until $32 \mathrm{~h}$ because the $\mathrm{pH}$ variation was only 0.3 within this period of time. The OCP test took $24 \mathrm{~h}$, the EIS test took around $35 \mathrm{~min}$, and the cyclic polarization test took around $5 \mathrm{~h}$ and $20 \mathrm{~min}$. 
Thus the $\mathrm{pH}$ change over $32 \mathrm{~h}$ can be considered to be acceptable when the time cost for one experiment was less than $30 \mathrm{~h}$.

Table 3-5 The $\mathrm{pH}$ exploration data of cyclohexylamine solution for $48 \mathrm{~h}$

\begin{tabular}{|c|c|c|c|c|c|c|c|c|c|c|}
\hline Time (h) & $\mathbf{0}$ & $\mathbf{0 . 5}$ & $\mathbf{1}$ & $\mathbf{2}$ & $\mathbf{4}$ & $\mathbf{7}$ & $\mathbf{2 4}$ & $\mathbf{2 7}$ & $\mathbf{3 2}$ & $\mathbf{4 8}$ \\
\hline $\mathbf{p H}$ & 9.69 & 9.79 & 9.79 & 9.79 & 9.78 & 9.71 & 9.60 & 9.49 & 9.39 & 8.90 \\
\hline
\end{tabular}

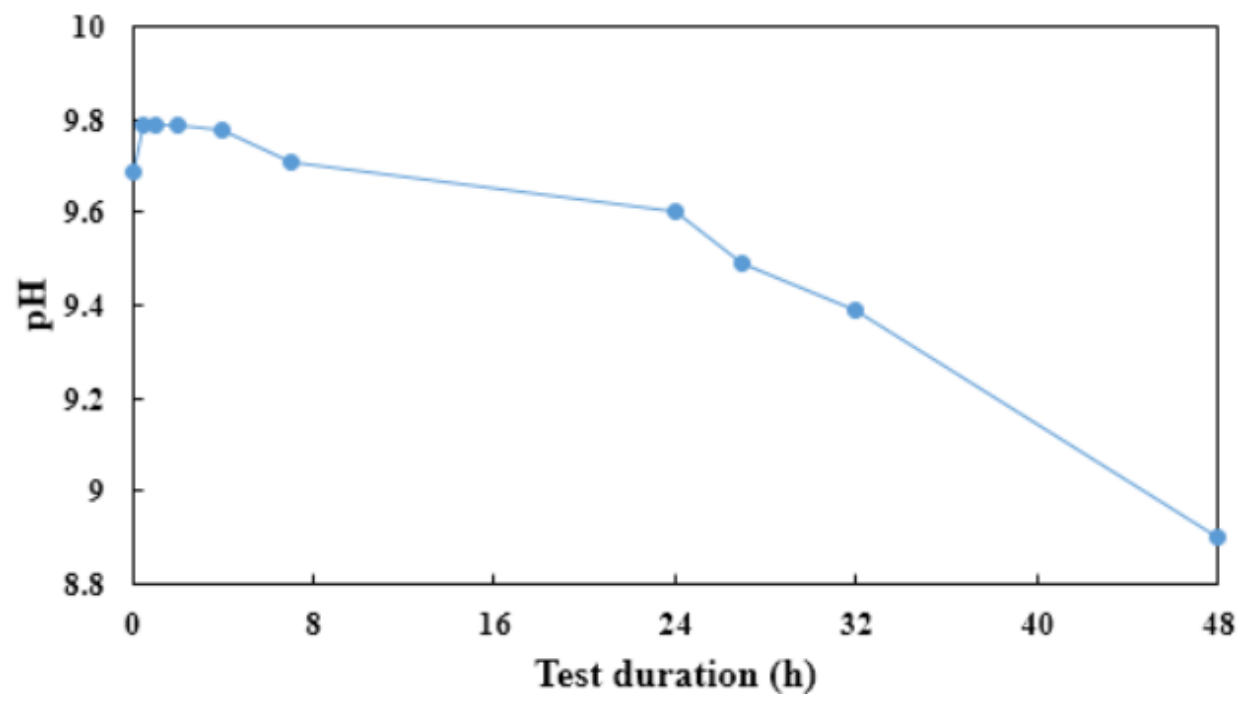

Figure 3-16 The pH exploration of cyclohexylamine solution for $48 \mathrm{~h}$

The $\mathrm{pH}$ variations were recorded five times for verification. The initial $\mathrm{pH}$ of the corrosion cell and the $\mathrm{pH}$ after $30 \mathrm{~h}$ were listed in Table 3-6. Although the $\mathrm{pH}$ of the cyclohexylamine solutions dropped every time, the original and final $\mathrm{pH}$ values were all around 9.5 if the initial $\mathrm{pH}$ was set to be slightly higher than 9.5 .

Table 3-6 The $\mathrm{pH}$ variation of cyclohexylamine solution

\begin{tabular}{|c|c|c|c|}
\hline & Initial pH & End pH & pH variation \\
\hline Test 1 & 9.69 & 9.41 & -0.28 \\
\hline Test 2 & 9.72 & 9.24 & -0.46 \\
\hline Test 3 & 9.74 & 9.49 & -0.25 \\
\hline Test 4 & 9.68 & 9.42 & -0.26 \\
\hline Test 5 & 9.70 & 9.40 & -0.30 \\
\hline
\end{tabular}




\subsubsection{Sodium hydroxide solution}

Since morpholine and cyclohexylamine are volatile amines, the $\mathrm{pH}$ must be maintained by the compensation system. However, sodium hydroxide solution is not volatile, the only issue it has is that the carbon dioxide in the air might go into the solution leading to a $\mathrm{pH}$ drop of the solution. If argon gas can keep bubbling the solution, there is no way for air to go into the solution. Several tests proved this theory, the initial $\mathrm{pH}$ and the final $\mathrm{pH}$ of the sodium hydroxide solution in the corrosion cell were almost the same after each experiment.

\subsection{System setups and procedures}

\subsubsection{Polarization test setup}

In this research, a Solartron 1287 potentiostat and 1255 frequency response analyzer (FRA), as illustrated in Figure 3-17 (a), and K0047 corrosion cell kit (Princeton Applied Research), shown in Figure 3-17 (b), were employed to conduct the electrochemical corrosion test. Corrware (3.2c) and Corrview (3.2d) software packages (Scribner Associates) were utilized for the (DC) related experiments, such as potentiodynamic and cyclic polarization tests. EIS was performed using Zplot (3.2c) and Zview (3.2d) software packages (Scribner Associates). Figure 3-17 (c) is a schematic diagram of the whole experimental system showing the combination of the electrochemical cell and the data acquisition and analysis system [23]. As can be seen clearly from the diagram, potentiostat and frequency response analyzer were used as a whole for transferring data from the corrosion cell to the computer. The waveform generator was facilitated inside as part of the Solartron 1287 electrochemical interface [63]. 


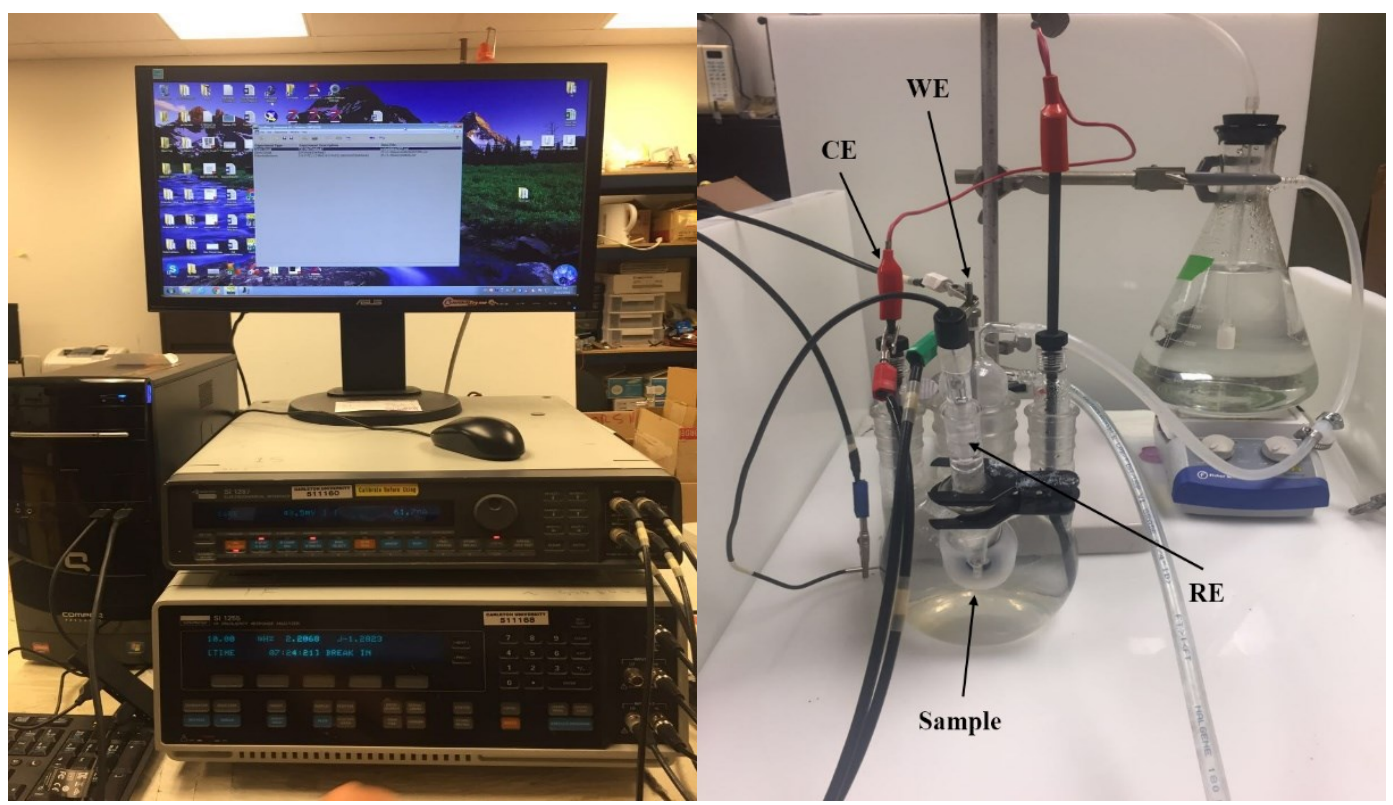

(a)

(b)

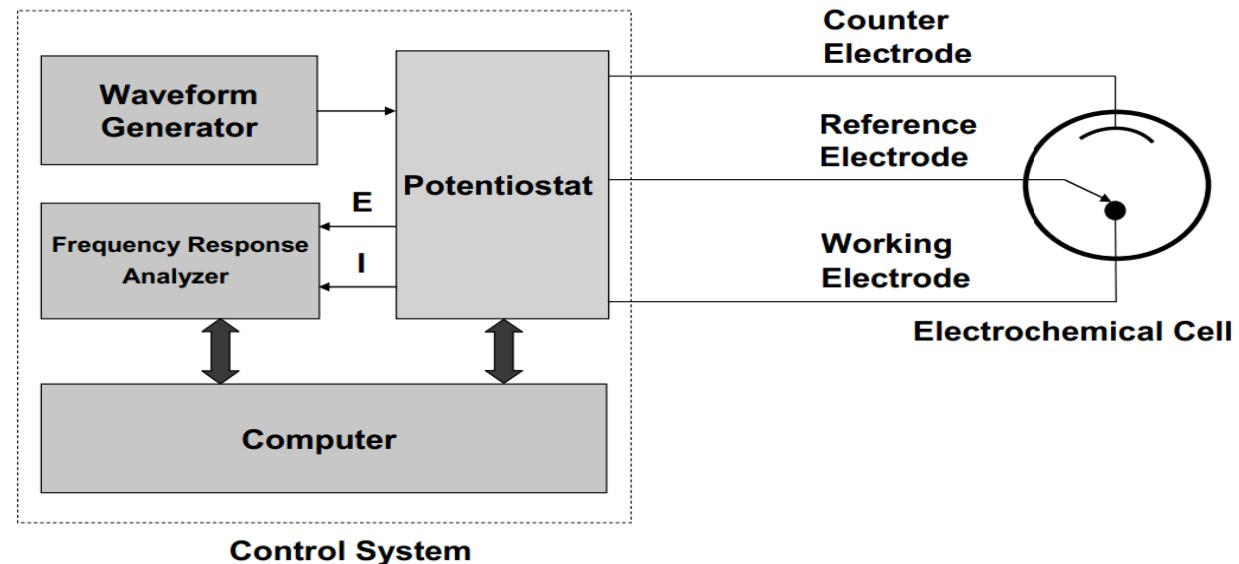

(c)

Figure 3-17 System setup: (a) Computer, Solartron 1287 potentiostat, and 1255 FRA; (b) K0047 corrosion cell kit with cable connections; (c) Schematic diagram of the electrochemical experimental setup [23]

However, individually, the function of potentiostat is mainly to keep the potential of the WE at a constant level with respect to the RE, the waveform generator is used to generate the electrical signal, the frequency response analyzer is used for providing a sinusoidal excitation signal and measuring the response to the previous self-provided sinusoidal signal as a ratio of one channel to the other and the computer is for collecting the data [63]. 
The corrosion cell consists of a CE (graphite), a RE (SCE), a WE (the sample which is mounted on the flat specimen holder). These three electrodes form a typical 3-terminal electrochemical cell [63], as shown in Figure 2-4. The electrodes are positioned following the instruction manual of K0047 corrosion cell kit, and Rosemary [89] gave a more clear discussion on the positions using K0047 corrosion cell kit, especially on using the flat specimen holder.

\subsubsection{Polarization test procedure}

At first, the system was aerated by argon gas for $30 \mathrm{~min}$ to get rid of oxygen thus to make the concentration of oxygen in the testing system small. At this stage, the pressure shown on the regulator must be adjusted to 2 psi, as demonstrated in Figure 3-18.

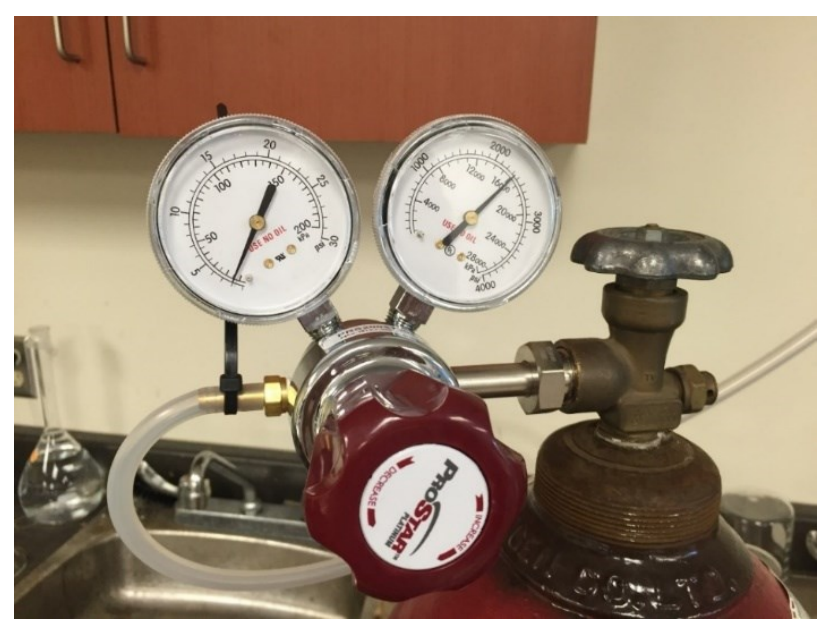

Figure 3-18 Pressure readings on regulator

The OCP test was performed before the polarization test. The test duration was set to be $24 \mathrm{~h}$ for the immersed specimen in the surrounding solution to reach a steady state. The steady state was characterized by the potential change which was considered to be less than 
$0.003 \mathrm{mV} / \mathrm{h}$ [66]. Since the cyclic polarization test was based on the potentiodynamic polarization test, the main reason to perform this test was to investigate the susceptibility of pitting or crevice corrosion of the metal in morpholine, cyclohexylamine and sodium hydroxide solutions [23]. The polarization test used in this thesis is a combination of normal potentiodynamic polarization test and cyclic polarization test. The setup of the potentiodynamic polarization is shown in Figure 3-19.

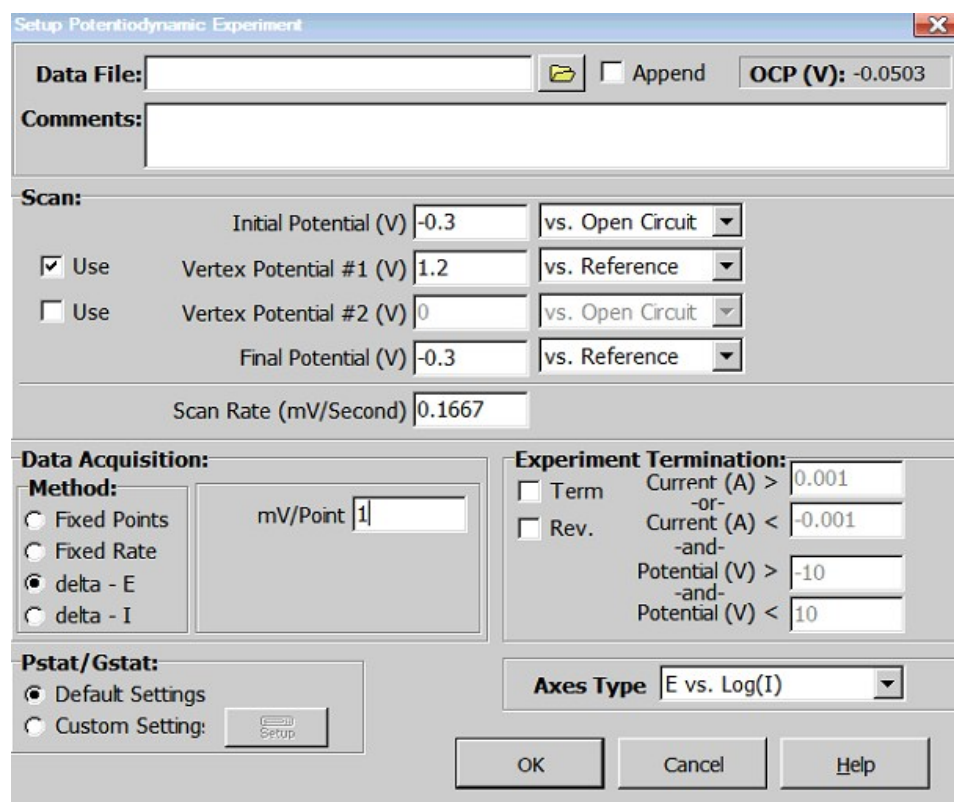

Figure 3-19 Potentiodynamic polarization setup

The polarization scan started from $-300 \mathrm{mV}$ vs. OCP, toward a predetermined point of $1200 \mathrm{mV}$ vs. reference, a reverse scan then started and ended at $-300 \mathrm{mV}$ vs. reference with the scan rate of $0.1667 \mathrm{mV} / \mathrm{s}[62,64,90]$. These starting and end points were chosen according to the ASTM standards G59 [79], G102 [65], G5 [80] and the practical circumstances of the tested materials and solution. The Tafel extrapolation must start at least $50 \mathrm{mV}$ to $100 \mathrm{mV}$ away from $E_{\text {corr }}$ to get an accurate extrapolation result [91]. The 
Tafel region (both or at least one branch of the Tafel curve) exhibits a linear characteristic on the semi-logarithmic scale over at least one decade of the current density.

\subsubsection{EIS test setup}

In three-electrode setup, the current leak through the RE has the ability to cause artifacts in the measured spectra. It is preferable to short the CE with the RE if a large RE impedance cannot be avoided. The shorting of the $\mathrm{CE}$ and the RE did not cause impedance artifacts in the EIS spectra. This method was proved in Anderson's research [92]. The concentration of the solutions in the corrosion cell and bridge tube (161.6 ppm of morpholine, $6 \mathrm{ppm}$ of cyclohexylamine, $2 \mathrm{ppm}$ of sodium hydroxide) can be considered to be low. Thus the impedance resulting from the low concentration is high enough to cause the artifacts. Therefore, the RE must be connected to the $\mathrm{CE}$ to avoid the artifacts, and the connections are shown in Figure 3-20. 


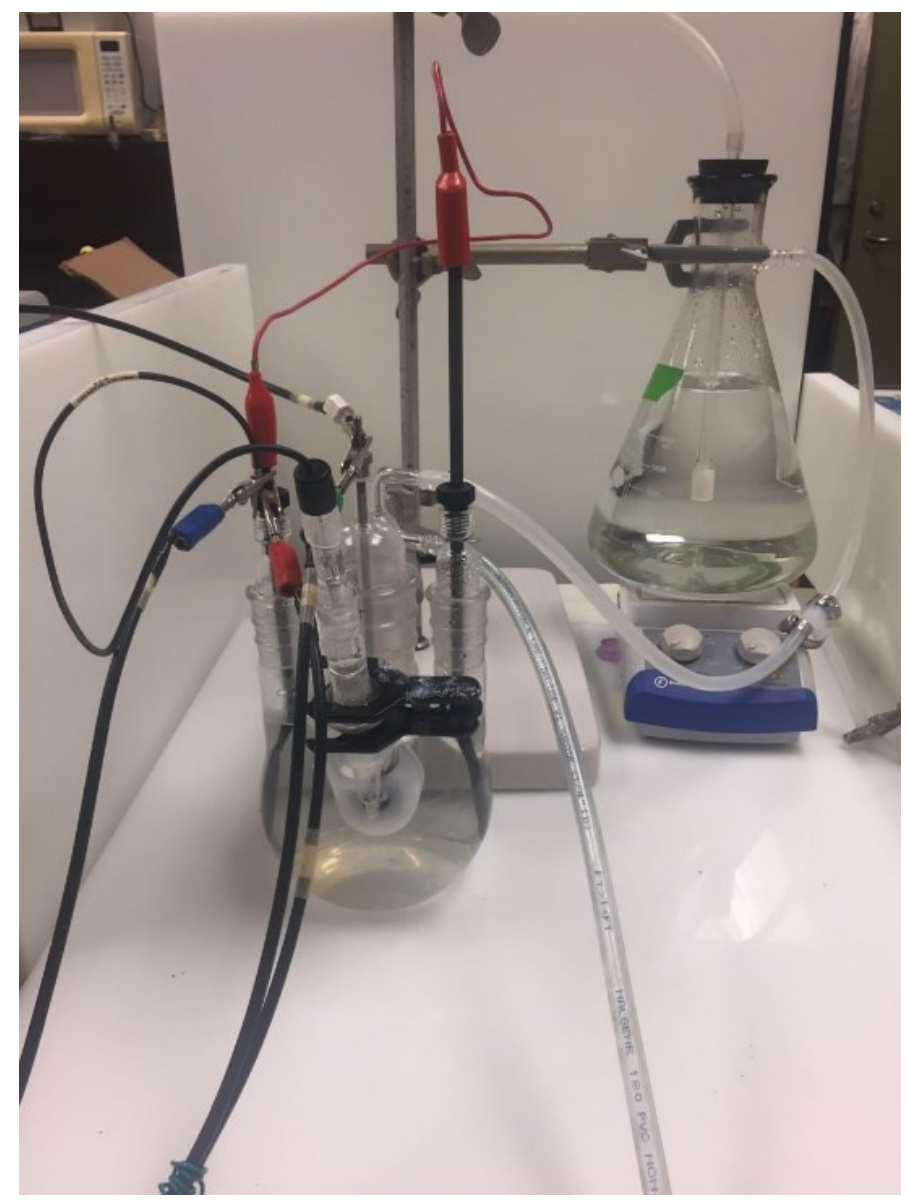

Figure 3-20 System setup of cable connections with RE and CE shorted

\subsubsection{EIS procedure}

The aeration process of $30 \mathrm{~min}$ and the test of OCP for $24 \mathrm{~h}$ are even more vital for the EIS method as EIS must start in the condition when a steady state of the system is satisfied [62]. The steady state is classified when the change of potential is less than $5 \mathrm{mV}$ in $10 \mathrm{~min}$ [91].

The Zplot setup for the EIS used in this research is illustrated in Figure 3-21. The DC potential was set to be $0 \mathrm{~V}$ vs. $\mathrm{OCP}$, and $\mathrm{AC}$ amplitude was chosen to be $10 \mathrm{mV}$. The $\mathrm{AC}$ signal was found to be 1 to $10 \mathrm{mV}$ in the tests because only a small potential signal could 
maintain the system pseudo-linear. If the system is non-linear, harmonic of excitation frequency may occur [62]. However, if the AC signals are too small, much noise may occur. The $10 \mathrm{mV}$ was decided as a combination of concerns about these two factors. The initial frequency was set to be $10 \mathrm{MHz}$ because the highest frequency limit of Solartron 1255 and 1287 package is $10 \mathrm{MHz}$. The final frequency was chosen to be $0.01 \mathrm{~Hz}$ as a balance of the test time and satisfactory EIS curve [66]. For example, the test duration of $27 \mathrm{~h}$ and $8 \mathrm{~min}$ may take for an EIS curve if the frequency range was chosen from $10^{-4} \mathrm{~Hz}$ to $10 \mathrm{MHz}$.

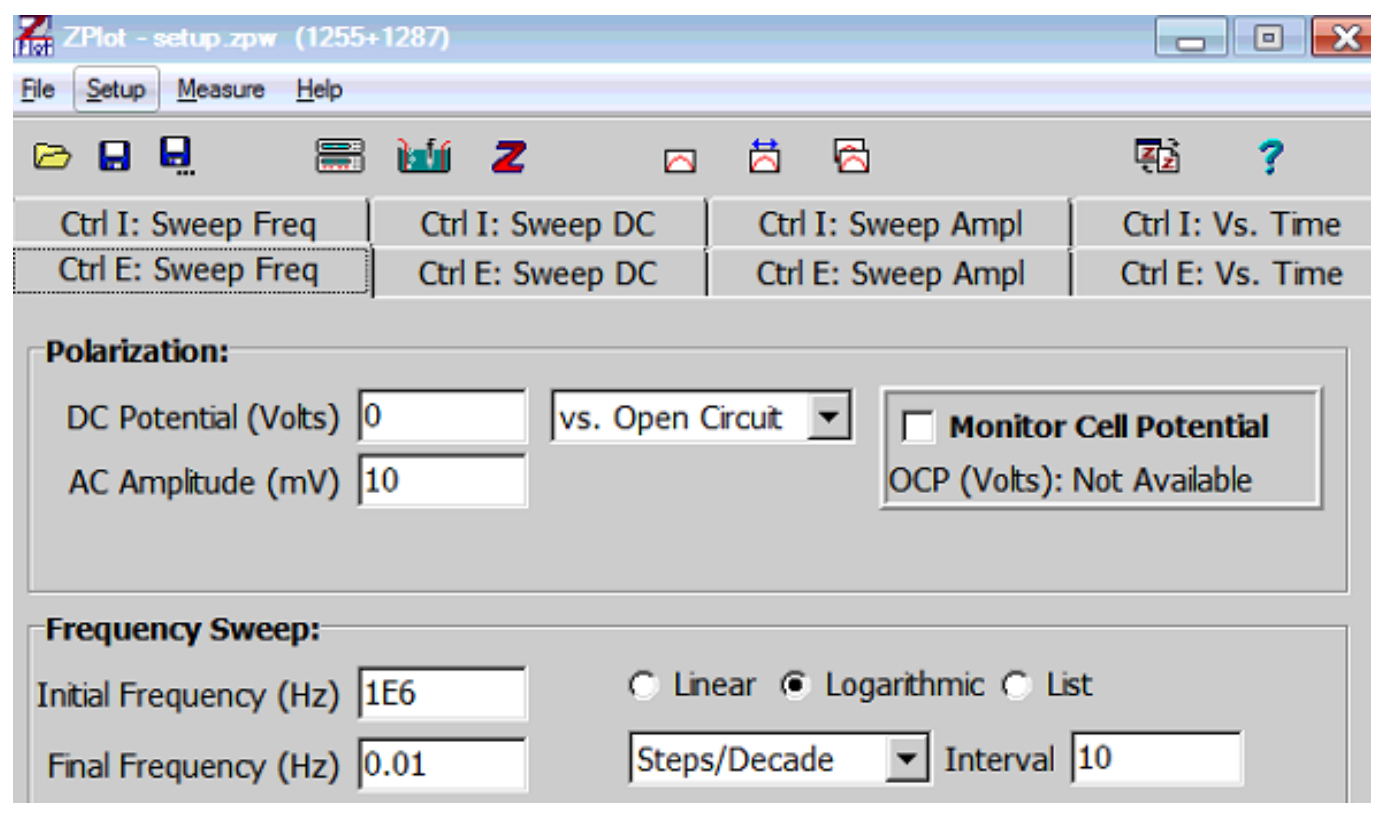

Figure 3-21 EIS Zplot setup 


\section{Chapter 4: Experimental Results and Analyses}

\subsection{Polarization test results}

Polarization tests were performed on Stellite 6 alloy and 17-4PH stainless steel in morpholine, cyclohexylamine and sodium hydroxide solutions at $\mathrm{pH}$ 9.5. Each test condition was repeated for three times to verify its consistency. For polarization curves, the $\mathrm{X}$-axis represents the logarithmic value of current density (base 10). The data were originally collected in the unit of an ampere, by making the original data ampere divided by specimen surface area, absolute values could be obtained for X-axis. The exposed surface area of the specimen was calculated to be $78.5 \mathrm{~mm}^{2}$ based on $\pi \cdot 5^{2} \mathrm{~mm}^{2}$. The $\mathrm{Y}$ axis represents the potential vs. SCE.

These results were all analyzed through the Corrview (3.2d) software. The corrosion potential $\left(E_{c o r r}\right)$, the current density $\left(I_{c o r r}\right)$ and the slope $\left(\beta_{a}\right.$ and $\left.\beta_{c}\right)$ that were required for Tafel exploration method can be obtained directly from the Corrview (3.2d) software. However, the polarization resistance $R_{p}$ had to be further calculated through the combination of Equation 2-5 to Equation 2-7. The numbers in parentheses are standard deviation errors in units of the least significant digit.

Normally, a large value of $E_{c o r r}$ indicates better corrosion resistance and a large value of $I_{\text {corr }}$ indicates a faster corrosion rate when corrosion starts. The polarization resistance $R_{p}$ is also a key factor that determines the corrosion behavior of the material. 


\subsubsection{Test results in morpholine solution}

\subsubsection{Potentiodynamic polarization}

Figure 4-1 and Figure 4-2 illustrate the polarization curves of Stellite 6 alloy and 17-4PH stainless steel in morpholine solution, respectively. The calculated values of parameters from the polarization curves are summarized in Table 4-1.

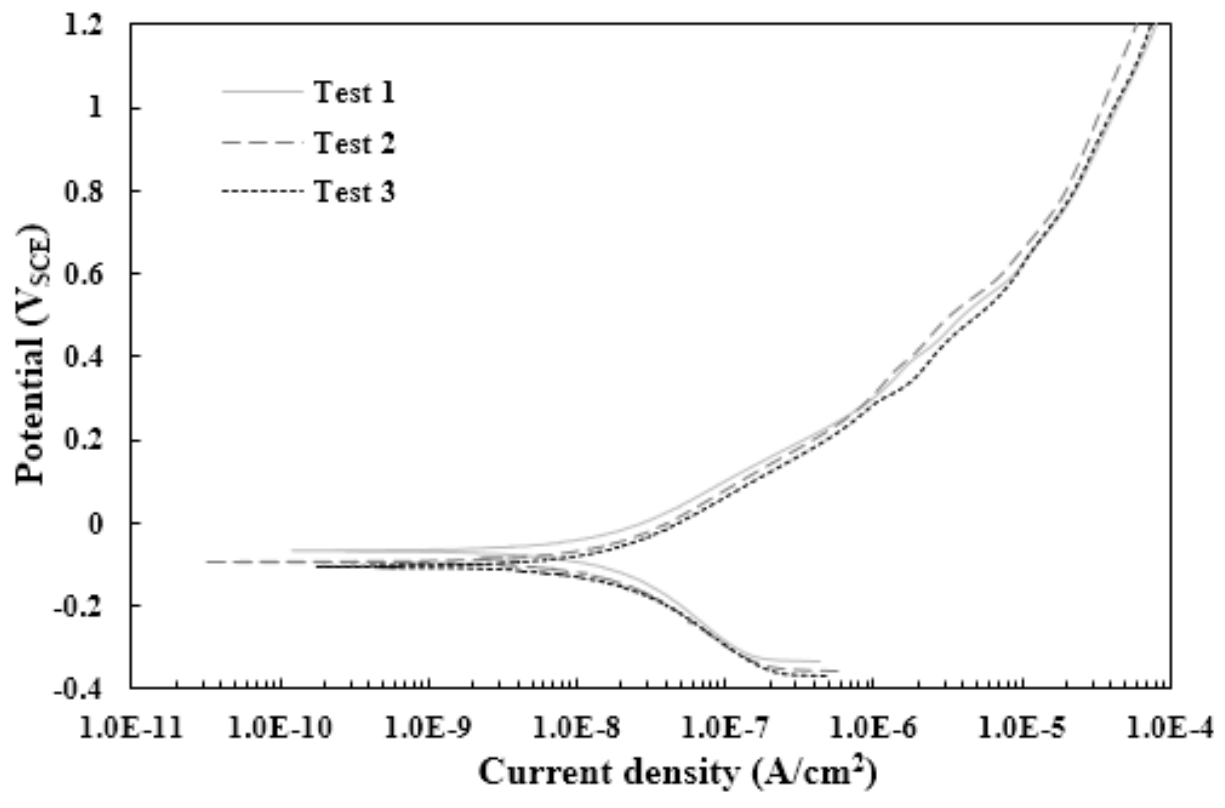

Figure 4-1 Polarization curves of Stellite 6 alloy in morpholine solution 


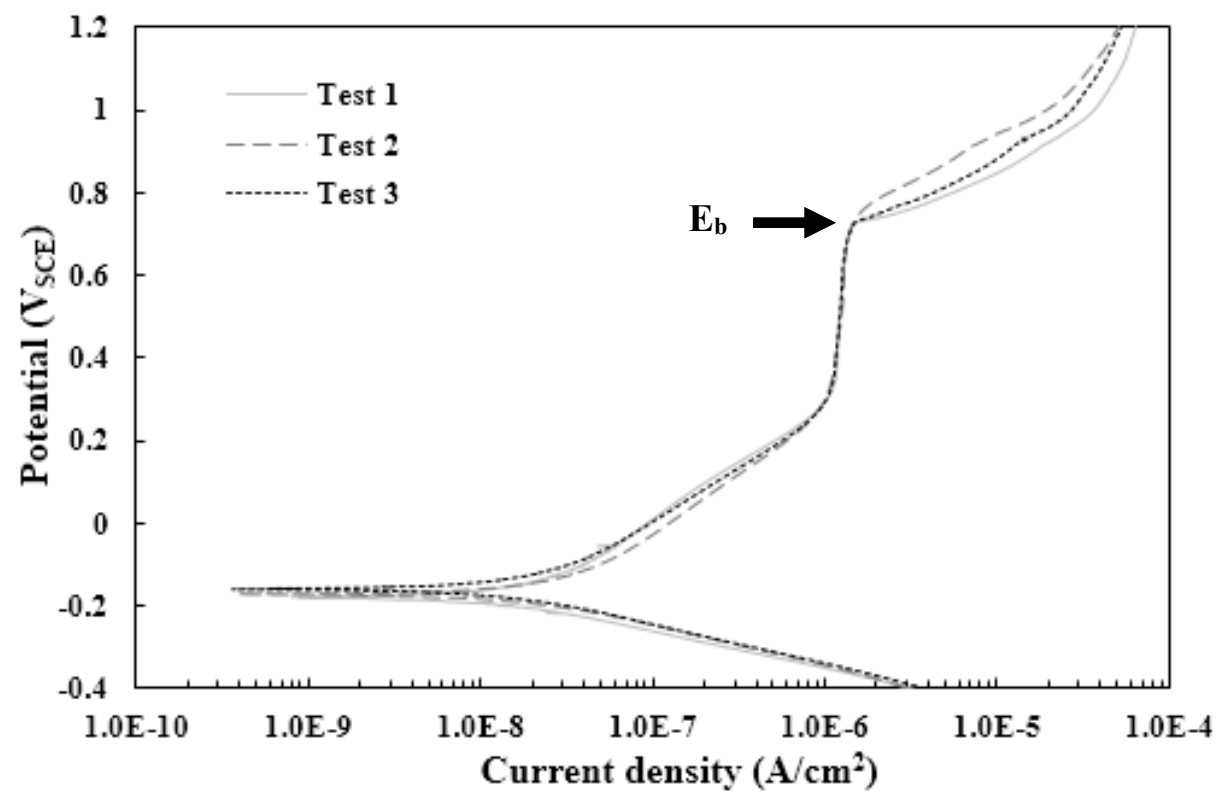

Figure 4-2 Polarization curves of 17-4PH stainless steel in morpholine solution

Table 4-1 Summary of polarization test results of Stellite 6 alloy and 17-4PH stainless steel in morpholine solution

\begin{tabular}{|c|c|c|c|c|c|c|c|c|}
\hline \multirow{2}{*}{ Specimen } & \multicolumn{4}{|c|}{ Stellite 6} & \multicolumn{4}{|c|}{ 17-4PH } \\
\hline & Test 1 & Test 2 & Test 3 & Average & Test 1 & Test 2 & Test 3 & Average \\
\hline $\begin{array}{c}E_{\text {corr }} \\
(V \text { vs. } S C E)\end{array}$ & -0.07 & -0.10 & -0.10 & $-0.09(2)$ & -0.18 & -0.17 & -0.16 & $-0.17(1)$ \\
\hline $\begin{array}{c}I_{\text {corr }} \\
\left(\mathrm{nA} / \mathbf{c m}^{2}\right)\end{array}$ & 21.1 & 20.7 & 20.5 & $20.8(3)$ & 19 & 27 & 19 & $22(5)$ \\
\hline $\begin{array}{c}R_{p} \\
\left(M \Omega \cdot \mathbf{c m}^{2}\right)\end{array}$ & 2.7 & 2.7 & 2.6 & $2.7(7)$ & 1.7 & 1.3 & 1.7 & $1.5(2)$ \\
\hline $\begin{array}{c}\beta_{a} \\
(V / \text { decade })\end{array}$ & 0.23 & 0.24 & 0.23 & $0.23(1)$ & 0.26 & 0.26 & 0.23 & $0.25(2)$ \\
\hline $\begin{array}{c}\boldsymbol{\beta}_{\mathrm{c}} \\
(\mathrm{V} / \mathrm{dec} \text { ede })\end{array}$ & 0.31 & 0.28 & 0.26 & $0.28(3)$ & 0.10 & 0.11 & 0.10 & $0.10(1)$ \\
\hline
\end{tabular}

For Stellite 6 alloy, as shown in Figure 4-1, within the tested potentials, Stellite 6 alloy is active, indicating that the metal oxidation proceeds continuously on the sample surface, which is the dominant reaction between the sample and morpholine solution. As a result, there is no evident passive region. However, as the potential increases, the slope of the 
polarization curve becomes sharper, which implies that passivation may occur. On the contrary, the polarization curve of $17-4 \mathrm{PH}$ stainless steel has an obvious passive region within the potential range from 0.2 to $0.8 \mathrm{~V}_{\mathrm{SCE}}$, as shown in Figure 4-2, indicating the formation of the protective oxide film on the sample surface. With raising the potential, beyond the passive region, the current starts to increase again, indicating the break of the oxide film. The breakaway potential $E_{b}$ occurs.

To further analyze the corrosion behavior of Stellite 6 alloy and 17-4PH stainless steel, one of the polarization curves was chosen as a representative and divided into several phases based on the slope of the curve for each tested material. A higher value of the slope $(\beta)$ indicates better similarity with the passive region, thus the material exhibits better corrosion resistance. As shown in Figure 4-3 and Figure 4-4, there are approximately three phases of the polarization curve for Stellite 6 alloy and four phases for 17-4PH stainless steel in morpholine solution. The slope of Stellite 6 continuously increases from phase 1 to phase 3, which means that it may have the passive region when an oxide film fully forms on its surface. For $17-4 \mathrm{PH}$ stainless steel, the slope of phase 2 is nearly infinite, representing the protective film formed on its surface. On the other hand, this passive region does not keep long and the slope decreases with the potential increasing, indicating the break of the oxide film. However, since the slope of phase 4 is larger than that of phase 3, this metal has the ability to repair the oxide film. 


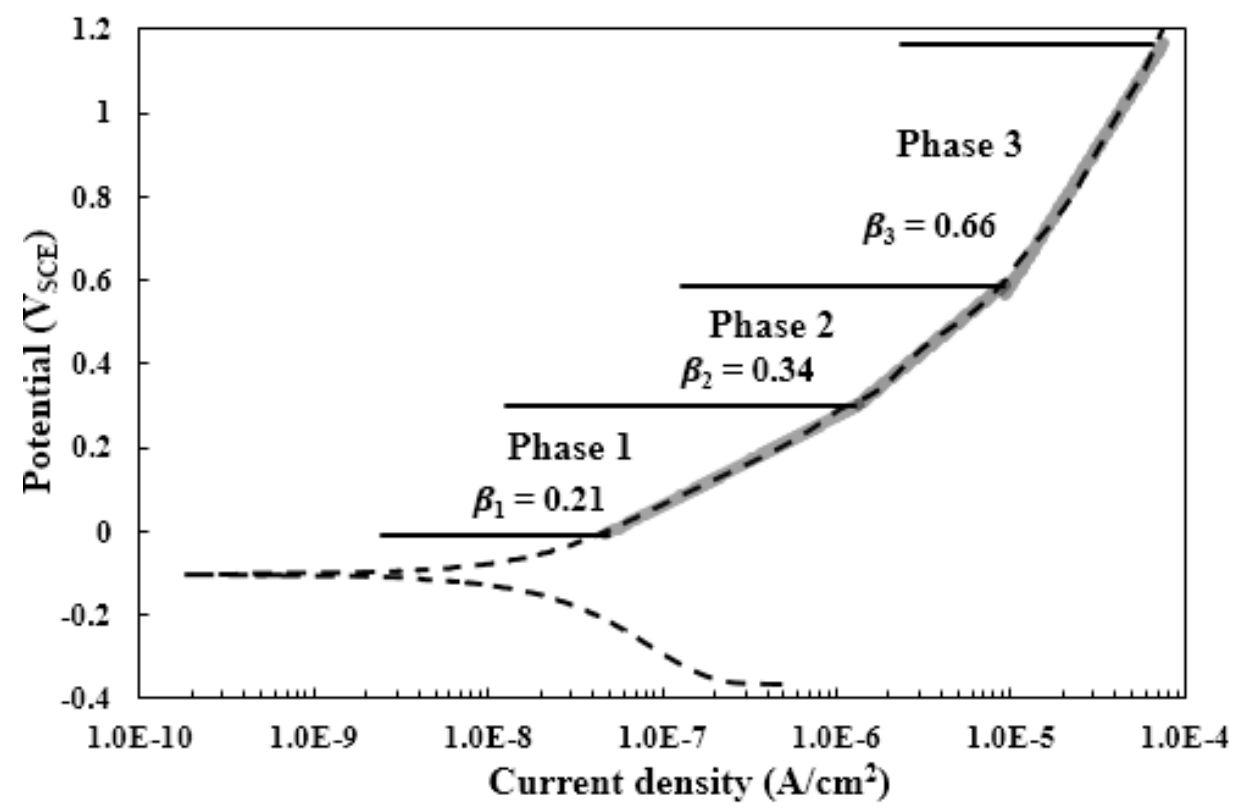

Figure 4-3 Divided phases of polarization curve of Stellite 6 alloy in morpholine solution

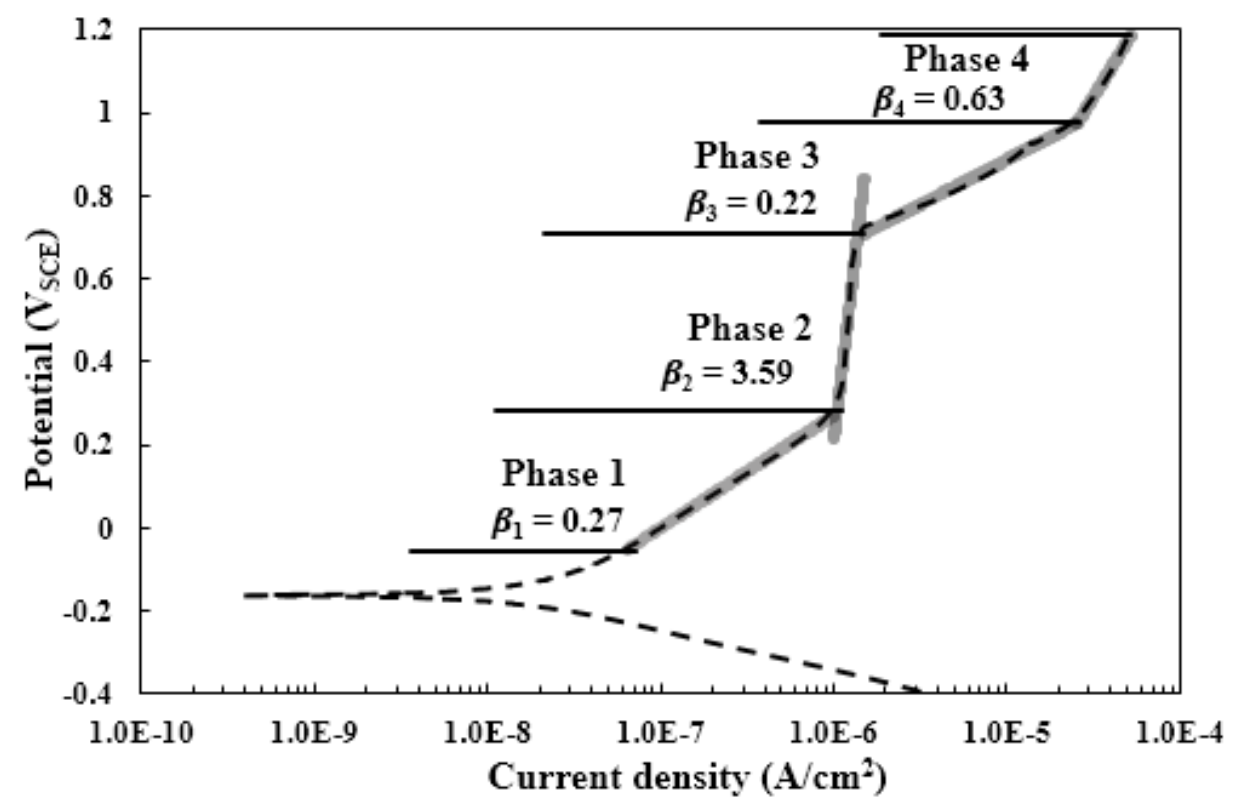

Figure 4-4 Divided phases of polarization curve of 17-4PH stainless steel in morpholine solution

It can be seen from Table 4-1 that the values of $E_{\text {corr }}$ of Stellite 6 alloy are larger than that of $17-4 \mathrm{PH}$ stainless steel so that Stellite 6 alloy is less easy to start oxidizing than $17-4 \mathrm{PH}$ 
stainless steel. Since the values of $I_{c o r r}$ for both Stellite 6 alloy and 17-4PH stainless steel are similar and the polarization resistance $R_{p}$ of Stellite 6 alloy is larger doubly than that of $17-4 \mathrm{PH}$ stainless steel, the former is more resistant than the latter to corrosion in morpholine solution.

\subsubsection{Cyclic polarization}

The cyclic polarization curves of three tests for both Stellite 6 alloy and 17-4PH stainless steel are very consistent. For better visualization, one of the cyclic polarization curves for Stellite 6 alloy and 17-4PH stainless steel in morpholine solution are plotted in Figure 4-5 and Figure 4-6, respectively. The dotted line of the curve represents the forward scan, and the solid line represents the reverse scan. The reverse scan is also called hysteresis loop. If the hysteresis loop is electropositive, that is, the current density of the backward scan is smaller than that of the forward scan at the same potential (Y-axis), the tested metal exhibits good localized corrosion (pitting corrosion) resistance. The material shows a better localized corrosion resistance when the hysteresis loop has a larger offset [23]. On the contrary, if the hysteresis loop is electronegative, the larger the offset, the worse the localized corrosion resistance of the material is [93, 94].

There is no hysteresis loop for Stellite 6 alloy, but 17-4PH stainless steel has a large electronegative hysteresis loop. Therefore, 17-4PH stainless steel is worse than Stellite 6 alloy with respect to localized (or pitting) corrosion in morpholine solution. 


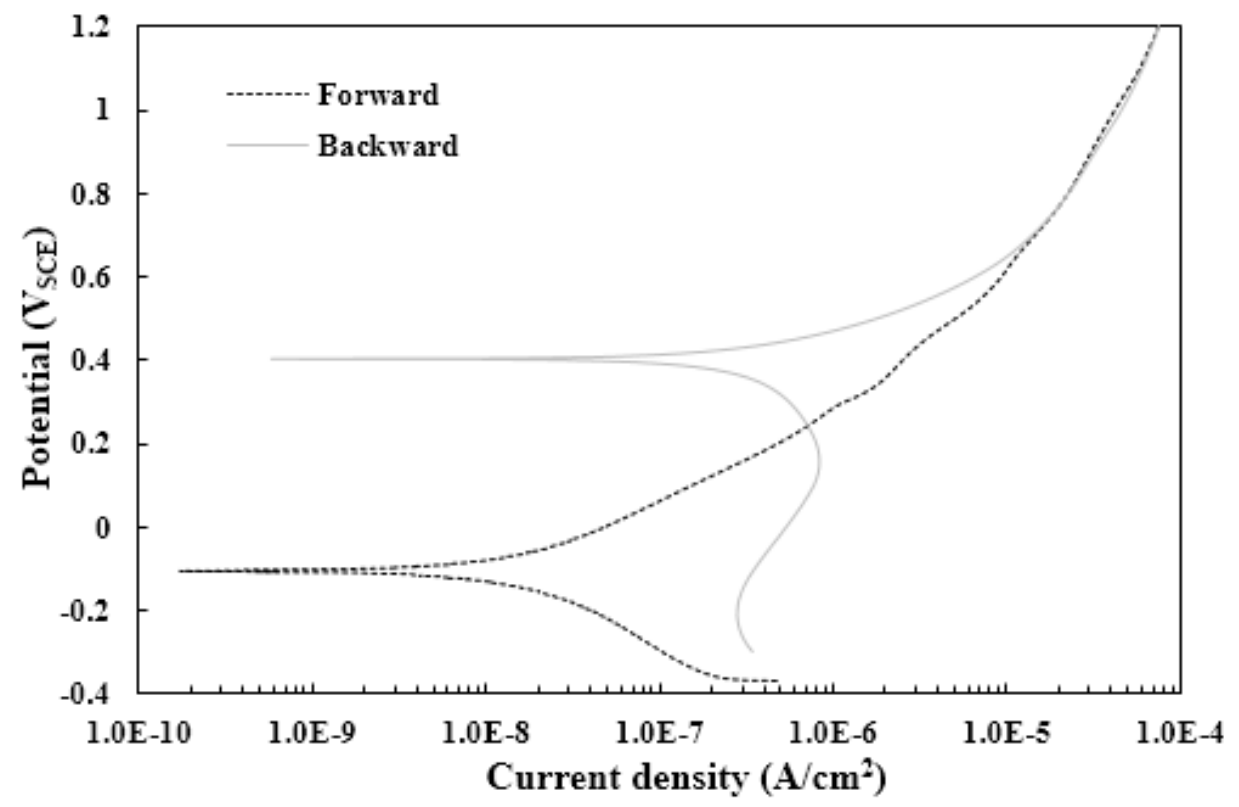

Figure 4-5 Cyclic polarization curve of Stellite 6 alloy in morpholine solution

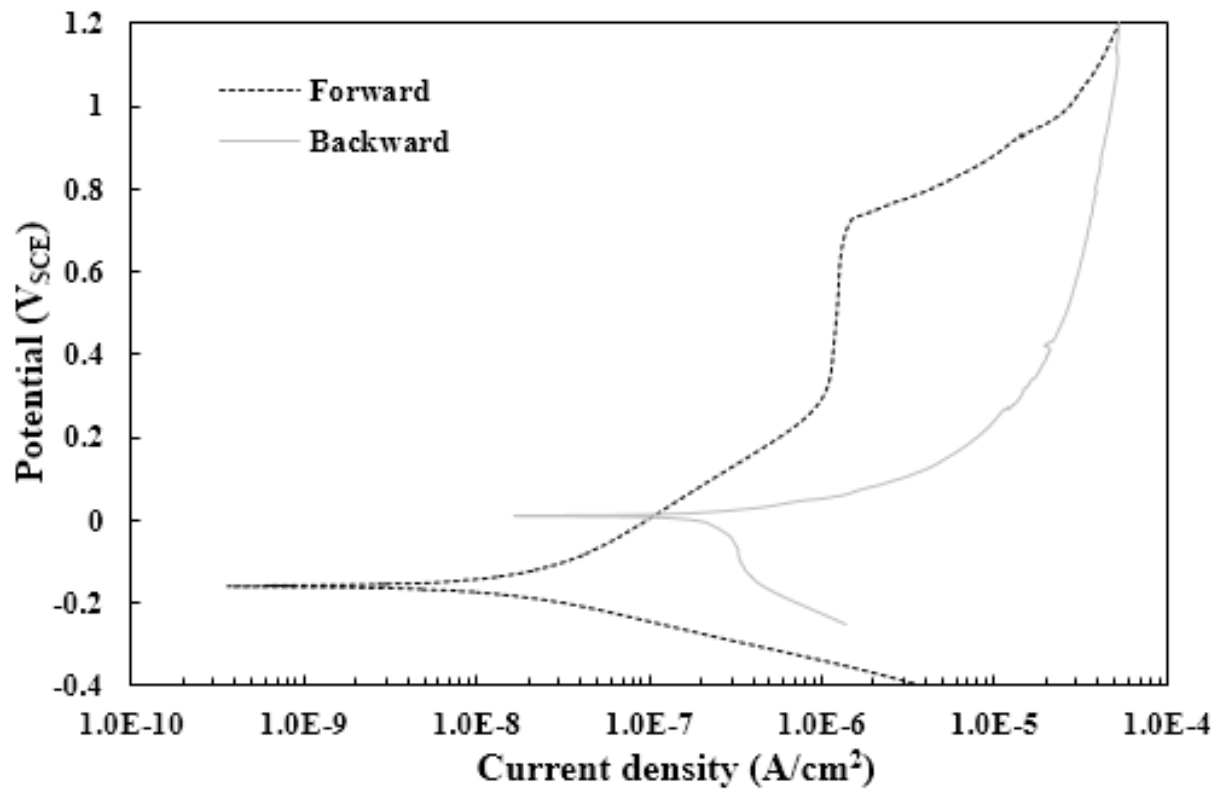

Figure 4-6 Cyclic polarization curve of 17-4PH stainless steel in morpholine solution 


\subsubsection{Test results in cyclohexylamine solution}

\subsubsection{Potentiodynamic polarization}

Figure 4-7 and Figure 4-8 plot the polarization curves of Stellite 6 alloy and 17-4PH stainless steel in cyclohexylamine solution, respectively. The calculated values of parameters from the polarization curves are summarized in Table 4-2.

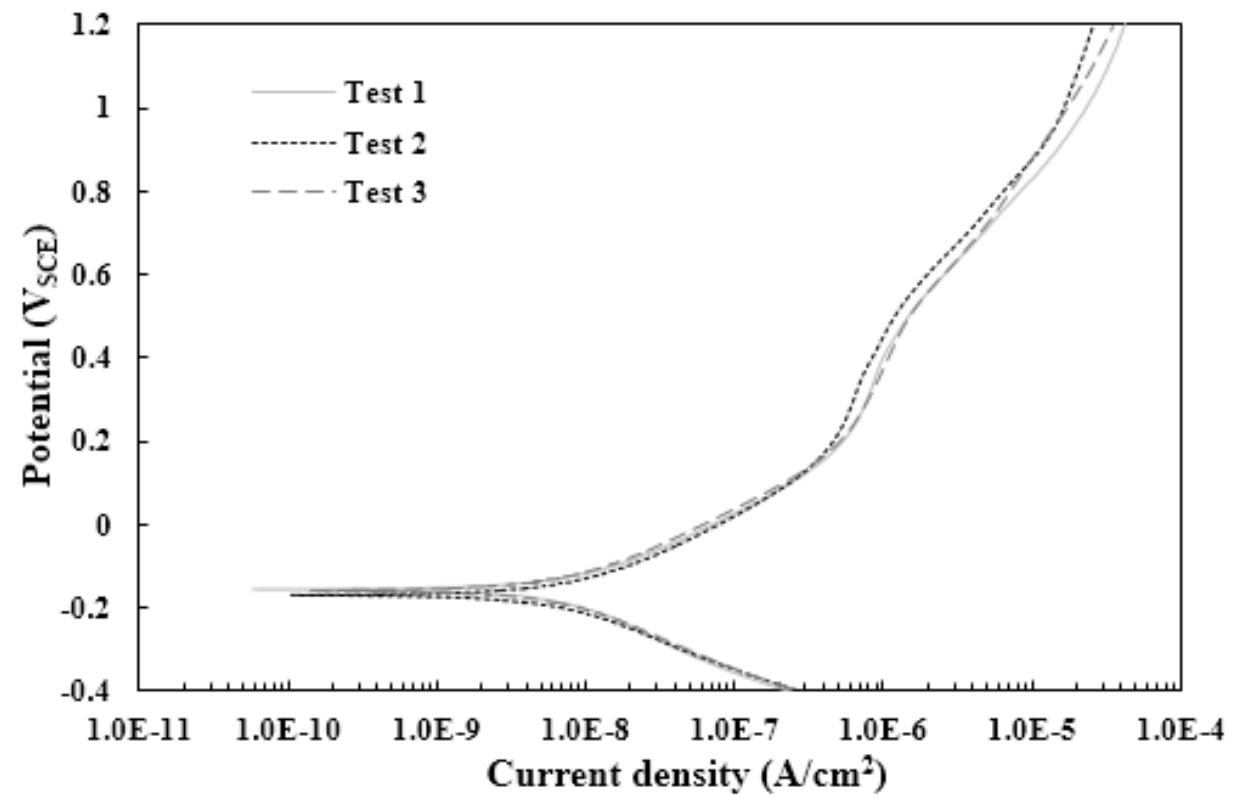

Figure 4-7 Polarization curves of Stellite 6 alloy in cyclohexylamine solution 


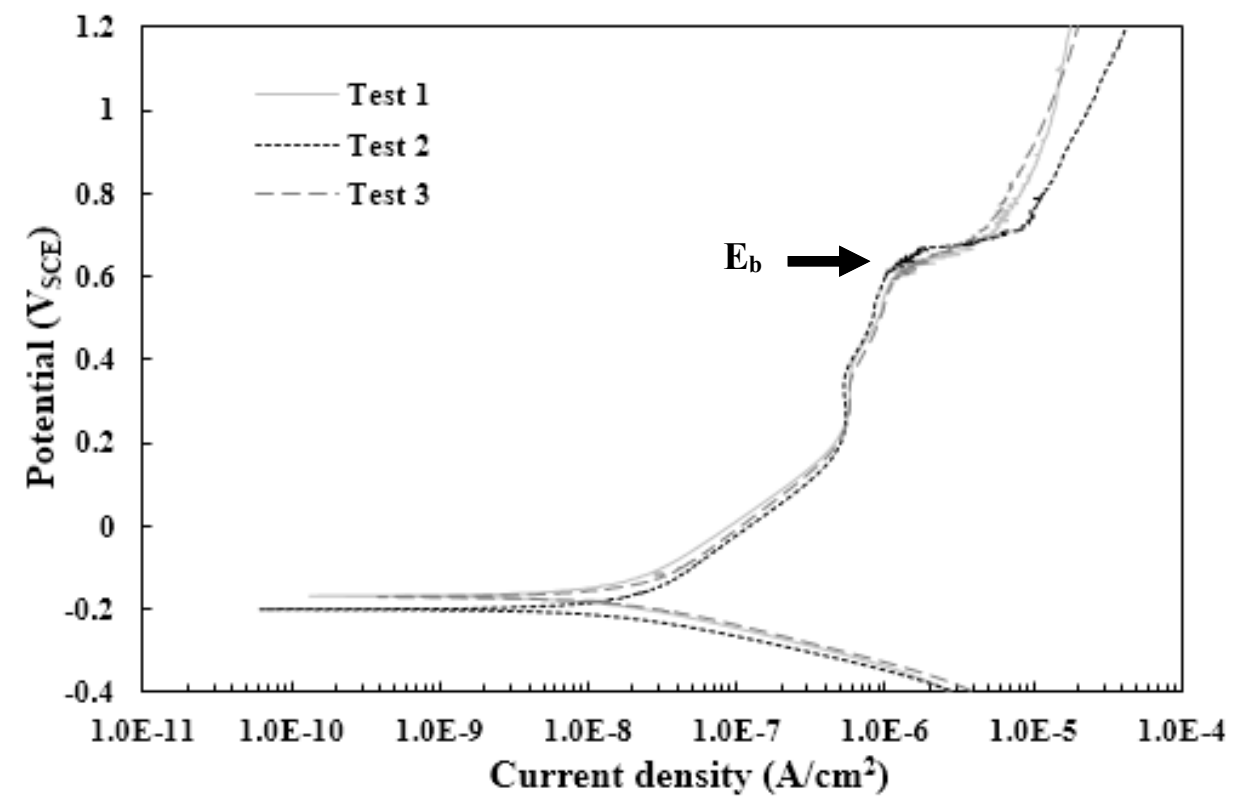

Figure 4-8 Polarization curves of 17-4PH stainless steel in cyclohexylamine solution

Table 4-2 Summary of polarization test results of Stellite 6 alloy and 17-4PH stainless steel in cyclohexylamine solution

\begin{tabular}{|c|c|c|c|c|c|c|c|c|}
\hline \multirow{2}{*}{ Specimen } & \multicolumn{4}{|c|}{ Stellite 6 } & \multicolumn{4}{c|}{ 17-4PH } \\
\cline { 2 - 9 } & Test 1 & Test 2 & Test 3 & Average & Test 1 & Test 2 & Test 3 & Average \\
\hline $\begin{array}{c}\mathbf{E}_{\mathbf{c o r r}} \\
(\mathbf{V} \text { vs. SCE) }\end{array}$ & -0.16 & -0.17 & -0.16 & $-0.16(1)$ & -0.17 & -0.20 & -0.17 & $-0.18(2)$ \\
$\begin{array}{c}\mathbf{I}_{\text {corr }} \\
\left(\mathbf{n A} / \mathbf{c m}^{2}\right)\end{array}$ & 8.1 & 7.3 & 7.1 & $7.5(5)$ & 21 & 27 & 28 & $25(4)$ \\
$\begin{array}{c}\mathbf{R}_{\mathbf{p}} \\
\left(\mathbf{M} \mathbf{\mathbf { c m } ^ { 2 }}\right)\end{array}$ & 4.7 & 4.7 & 5.0 & $4.8(2)$ & 1.6 & 1.2 & 1.2 & $1.3(2)$ \\
\hline $\begin{array}{c}\boldsymbol{\beta}_{\mathbf{a}} \\
(\mathbf{V} / \mathbf{d e c a d e})\end{array}$ & 0.170 & 0.170 & 0.172 & $0.171(1)$ & 0.26 & 0.31 & 0.29 & $0.29(2)$ \\
\hline $\begin{array}{c}\boldsymbol{\beta}_{\mathbf{c}} \\
(\mathbf{V} / \mathbf{d e c a d e})\end{array}$ & 0.18 & 0.15 & 0.16 & $0.16(2)$ & 0.106 & 0.102 & 0.105 & $0.104(2)$ \\
\hline
\end{tabular}

The divided phases of the polarization curves for this test condition are illustrated in Figure 4-9 and Figure 4-10. Stellite 6 alloy and 17-4PH stainless steel, respectively, exhibit similar behavior in morpholine solution and in cyclohexylamine solution. 


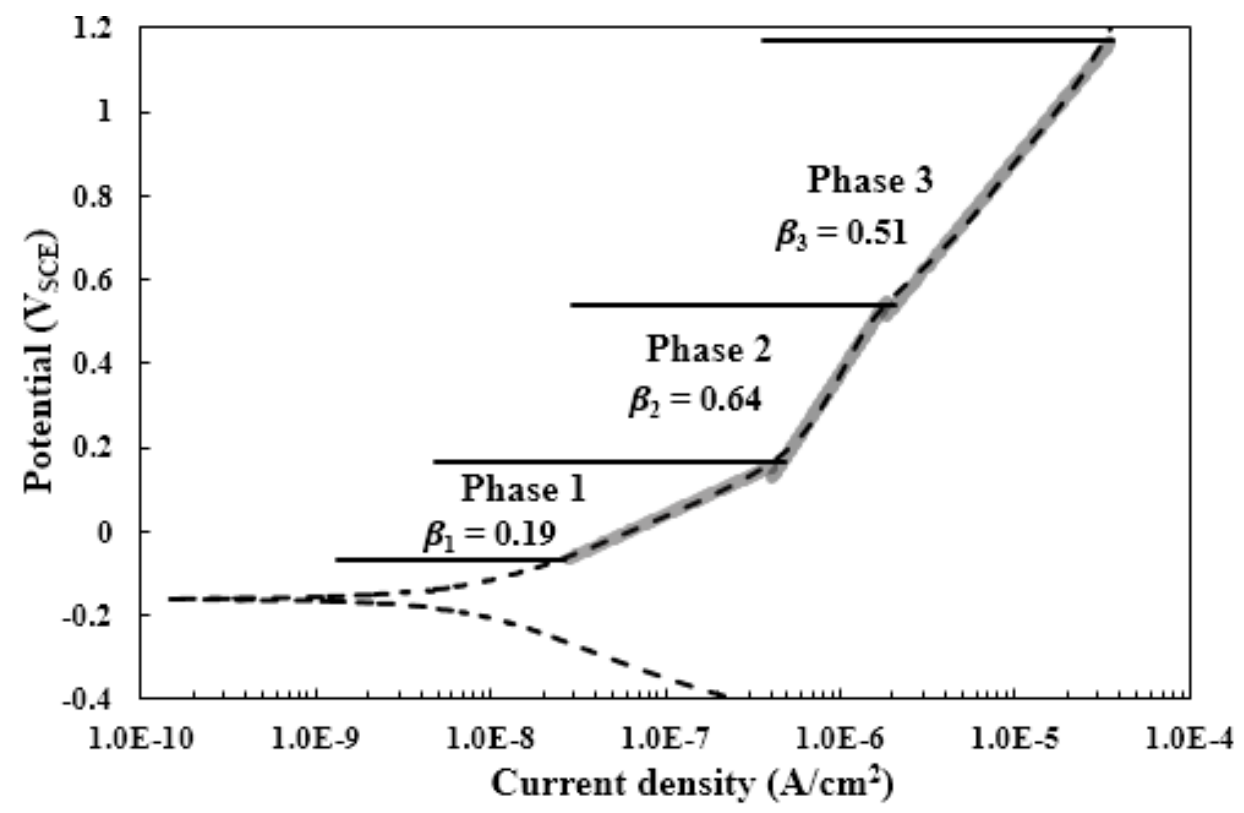

Figure 4-9 Divided phases of polarization curve of Stellite 6 alloy in cyclohexylamine solution

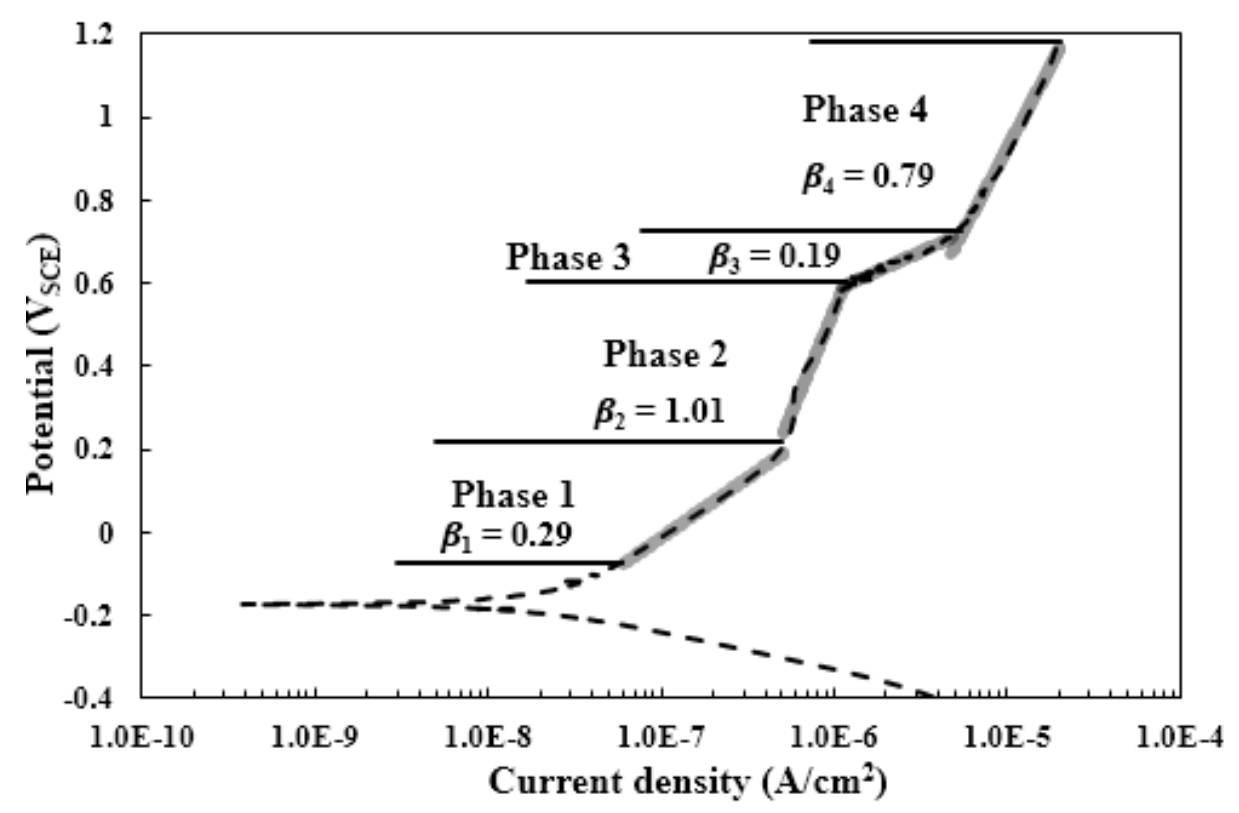

Figure 4-10 Divided phases of polarization curve of 17-4PH stainless steel in cyclohexylamine solution

In addition, the values of $E_{\text {corr }}$ for Stellite 6 alloy and 17-4PH stainless steel are similar. The values of $I_{\text {corr }}$ of Stellite 6 alloy are smaller than that of 17-4PH stainless steel and the 
polarization resistance $R_{p}$ of Stellite 6 alloy is much larger than that of 17-4PH stainless steel. Therefore, overall Stellite 6 is better than 17-4PH stainless steel in resisting corrosion to cyclohexylamine solution.

\subsubsection{Cyclic polarization}

For the same reason as for morpholine solution, one of the cyclic polarization curves of Stellite 6 alloy and 17-4PH stainless steel tested in cyclohexylamine solution are plotted in Figure 4-11 and Figure 4-12, respectively. Compared with the cyclic polarization curves for morpholine solution, the cyclic polarization curves for cyclohexylamine solution show that these two metals, respectively, behave similarly in the two amine solutions for pitting corrosion.

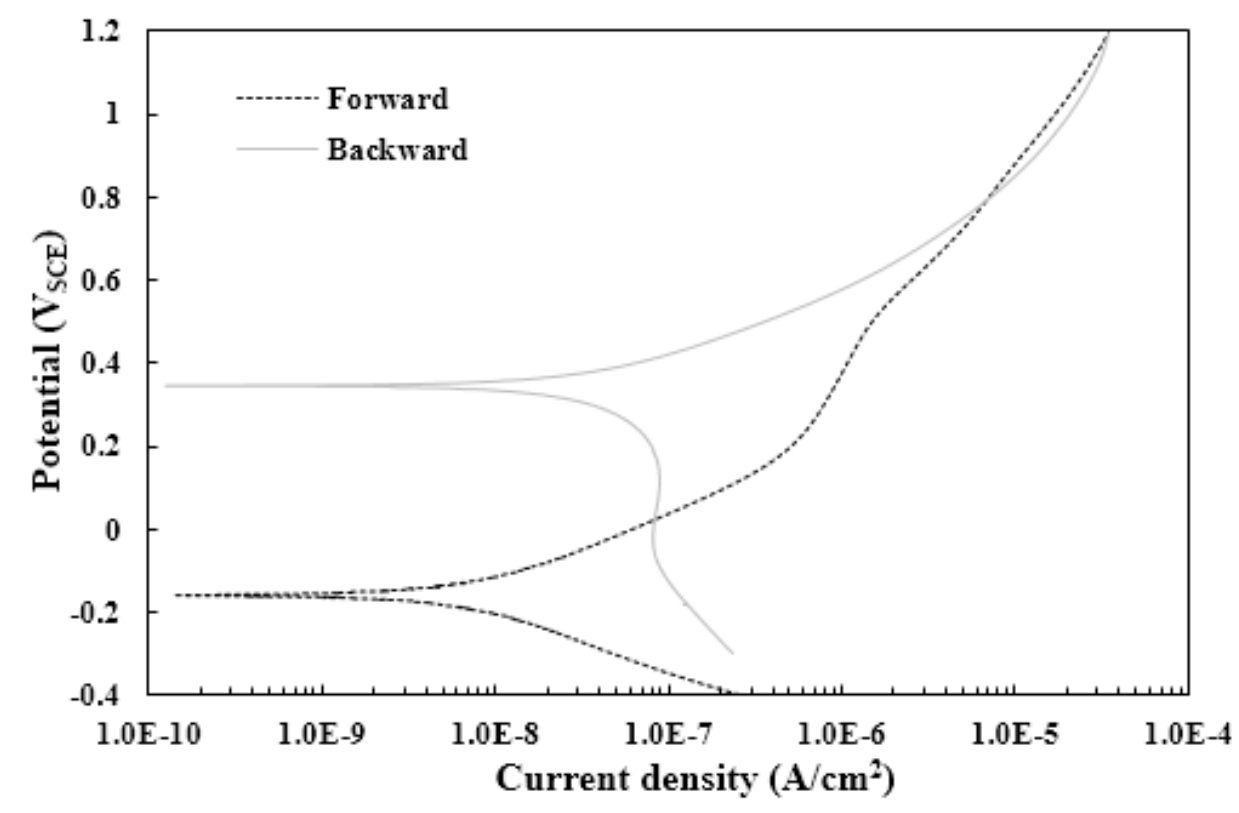

Figure 4-11 Cyclic polarization curve of Stellite 6 alloy in cyclohexylamine solution 


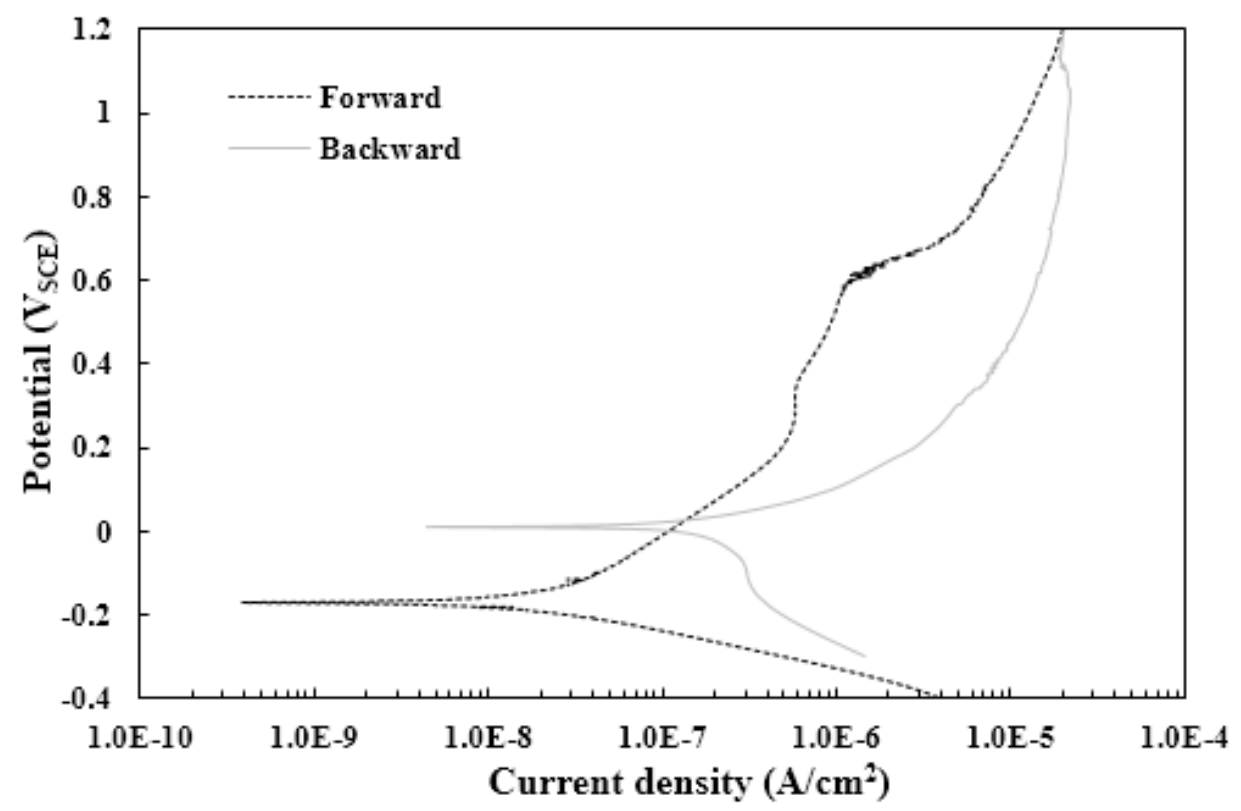

Figure 4-12 Cyclic polarization curve of 17-4PH stainless steel in cyclohexylamine solution

\subsubsection{Test results in sodium hydroxide solution}

\subsubsection{Potentiodynamic polarization}

Figure 4-13 and Figure 4-14 present the polarization curves of Stellite 6 alloy and 17-4PH stainless steel tested in sodium hydroxide solution, respectively. The calculated values of parameters from the polarization curves are summarized in Table 4-3. 


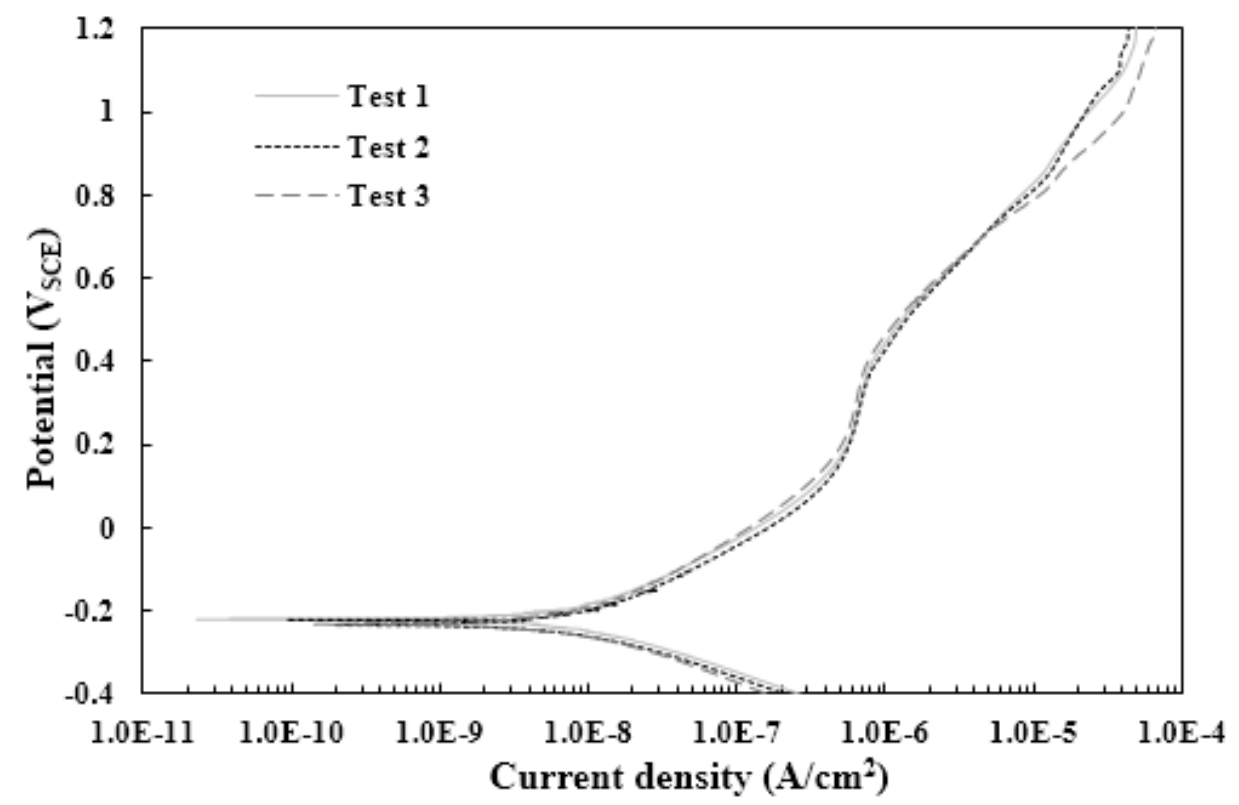

Figure 4-13 Polarization curves of Stellite 6 alloy in sodium hydroxide solution

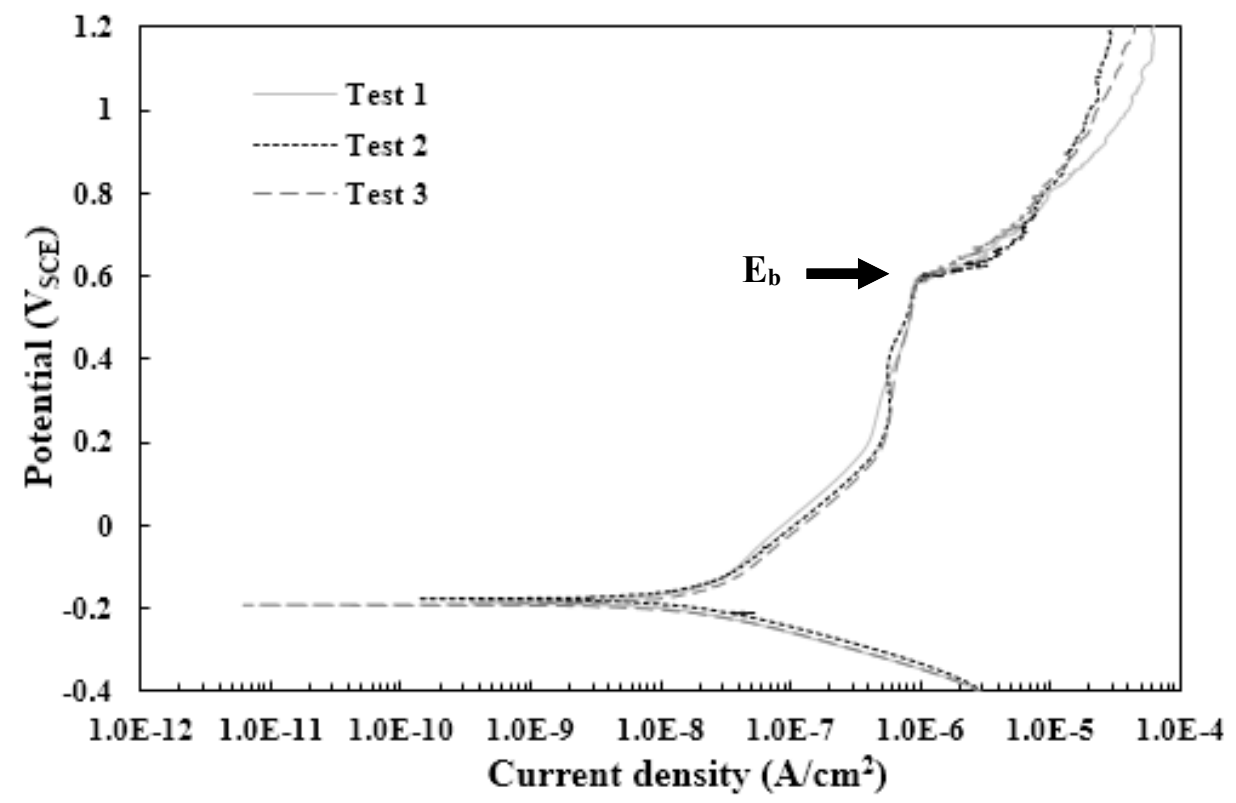

Figure 4-14 Polarization curves of 17-4PH stainless steel in sodium hydroxide solution 
Table 4-3 Summary of polarization test results of Stellite 6 alloy and 17-4PH stainless steel in sodium hydroxide solution

\begin{tabular}{|c|c|c|c|c|c|c|c|c|}
\hline \multirow{2}{*}{ Specimen } & \multicolumn{4}{|c|}{ Stellite 6 } & \multicolumn{4}{c|}{ 17-4PH } \\
\cline { 2 - 9 } & Test 1 & Test 2 & Test 3 & Average & Test 1 & Test 2 & Test 3 & Average \\
\hline $\begin{array}{c}\mathbf{E}_{\text {corr }} \\
(\mathbf{V ~ v s . ~ S C E )}\end{array}$ & -0.22 & -0.24 & -0.23 & $-0.23(1)$ & -0.19 & -0.18 & -0.19 & $-0.19(1)$ \\
\hline $\begin{array}{c}\mathbf{I}_{\text {corr }} \\
\left(\mathbf{n A} / \mathbf{c m}^{2}\right)\end{array}$ & 10.0 & 10.2 & 10.0 & $10.1(1)$ & 22 & 28 & 27 & $25(3)$ \\
\hline $\begin{array}{c}\mathbf{R}_{\mathbf{p}} \\
\left(\mathbf{M} \mathbf{\mathbf { c m } ^ { 2 }} \mathbf{c m}^{\mathbf{2}}\right)\end{array}$ & 3.3 & 3.3 & 3.6 & $3.4(2)$ & 1.5 & 1.2 & 1.2 & $1.3(2)$ \\
\hline $\begin{array}{c}\boldsymbol{\beta}_{\mathbf{a}} \\
(\mathbf{V} / \mathbf{d e c a d e})\end{array}$ & 0.19 & 0.19 & 0.21 & $0.20(1)$ & 0.31 & 0.30 & 0.30 & $0.30(1)$ \\
\hline $\begin{array}{c}\boldsymbol{\beta}_{\mathbf{c}} \\
(\mathbf{V} / \mathbf{d e c a d e})\end{array}$ & 0.12 & 0.13 & 0.14 & $0.13(1)$ & 0.102 & 0.108 & 0.106 & $0.105(3)$ \\
\hline
\end{tabular}

According to the polarization curves, Stellite 6 alloy and 17-4PH stainless steel, respectively, in general, behave the same in cyclohexylamine solution and in sodium hydroxide solution, but differences can be found from Figure 4-15 and Figure 4-16, the polarization curves of both Stellite 6 alloy and 17-4PH stainless steel have four phases. Like 17-4PH stainless steel, Stellite 6 in this solution exhibits passive behavior and the ability of oxide film repair.

From Table 4-3, the values of $E_{\text {corr }}$ for Stellite 6 alloy are slightly smaller than that of $17-4 \mathrm{PH}$ stainless steel, the values of $I_{\text {corr }}$ of Stellite 6 alloy are smaller than that of 17-4PH stainless steel, and the polarization resistance $R_{p}$ of Stellite 6 alloy is larger doubly than that of 17-4PH stainless steel. Therefore, Stellite 6 has better resistance to corrosion in sodium hydroxide solution. 


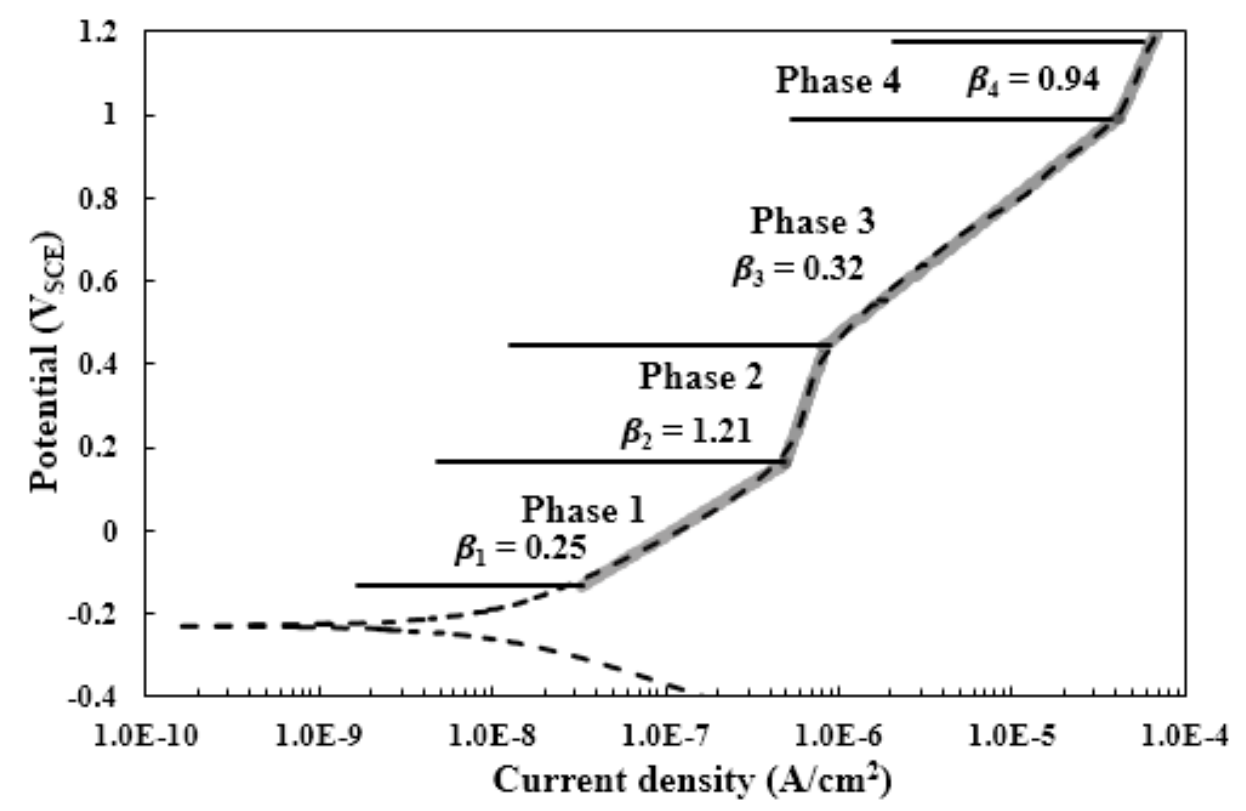

Figure 4-15 Divided phases of polarization curve of Stellite 6 alloy in sodium hydroxide solution

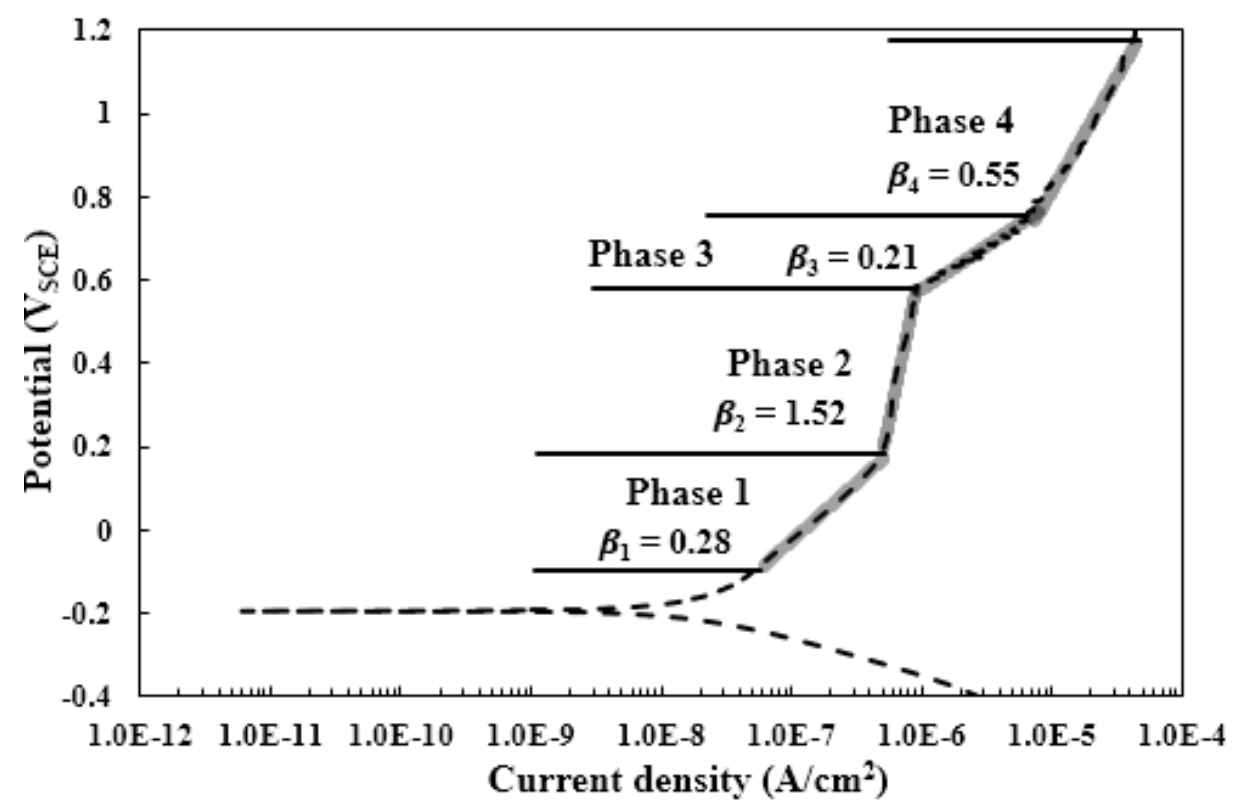

Figure 4-16 Divided phases of polarization curve of 17-4PH stainless steel in sodium hydroxide solution 


\subsubsection{Cyclic polarization}

Similarly, one of the cyclic polarization curves of Stellite 6 alloy and 17-4PH stainless steel tested in sodium hydroxide solution are presented in Figure 4-17 and Figure 4-18, respectively. For this solution, there is a little hysteresis loop on the Stellite 6 alloy curve, but the 17-4PH stainless steel curve still has a large electronegative hysteresis loop. Therefore, Stellite 6 alloy is also better than 17-4PH stainless steel in resisting localized corrosion to sodium hydroxide solution.

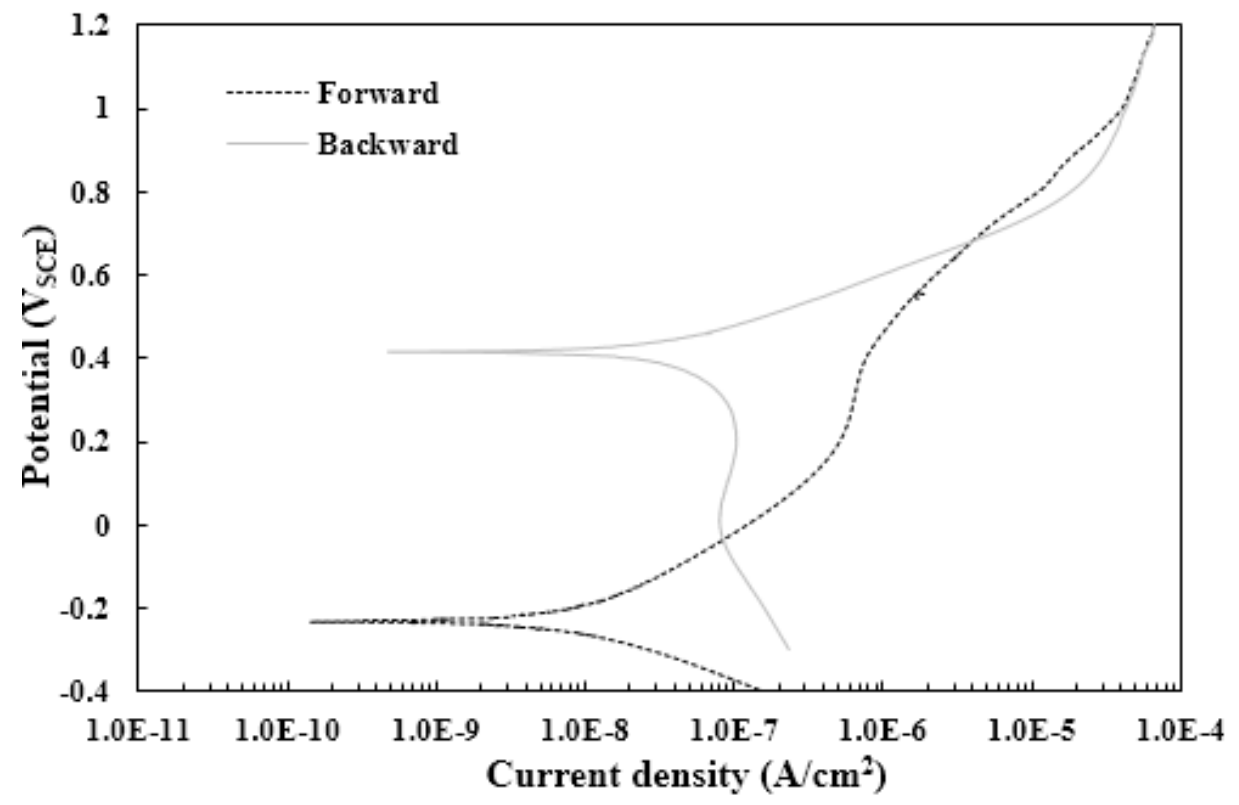

Figure 4-17 Cyclic polarization curve of Stellite 6 alloy in sodium hydroxide solution 


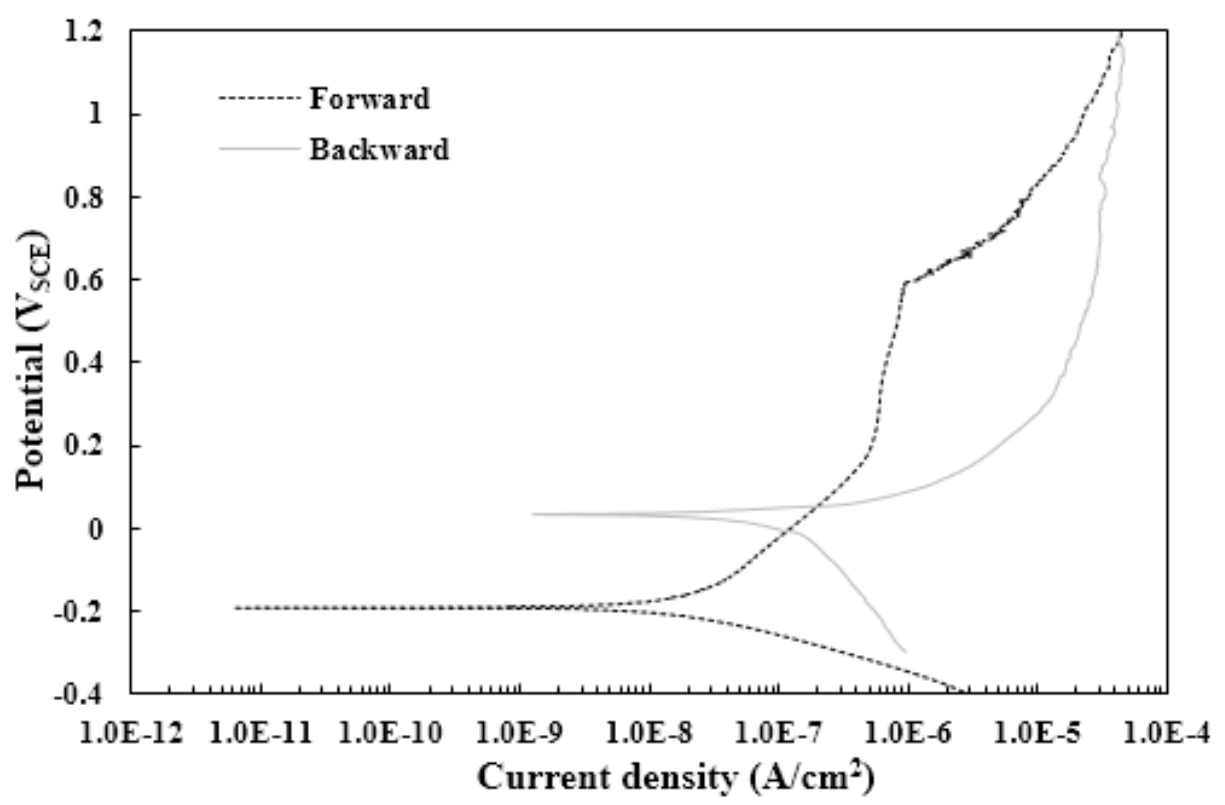

Figure 4-18 Cyclic polarization curve of 17-4PH stainless steel in sodium hydroxide solution

\subsection{EIS results}

Similar to polarization and cyclic polarization tests, three specimens of each material were subjected to EIS test in morpholine, cyclohexylamine and sodium hydroxide solutions, respectively. As illustrated in Figure 2-9, model 1 is more preferable to fit the EIS data when the protective oxide layers are not very homogeneous. Thus the equivalent circuit (Figure 4-19) was chosen to fit the EIS data, where $R_{s}$ is the solution resistance; $R_{f}$ and $R_{c t}$ are the oxide film and charge-transfer resistance respectively; $Q_{f}$ and $Q_{d l}$ represent the oxide film CPE and double-layer CPE respectively; $\alpha_{1}$ and $\alpha_{2}$ are two parameters which are related to $Q_{f}$ and $Q_{d l}$ [95]. A higher $\alpha$ value means that the CPE or $Q$ exhibits a more likely property of a capacitance. A higher $R_{f}$ indicates less possibility for the corrosion medium to penetrate the oxide film. The element $R_{c t}$ is the key factor that affects the corrosion resistance of the material in such a corrosion medium. However, as mentioned 
previously, not only one equivalent circuit can be used to fit the data, there can be other equivalent circuits showing good fitting results and reasonable fitting errors. The chosen equivalent circuit is one of the equivalent circuits with good fitting results and relatively fewer components included to interpret the corrosion behavior.

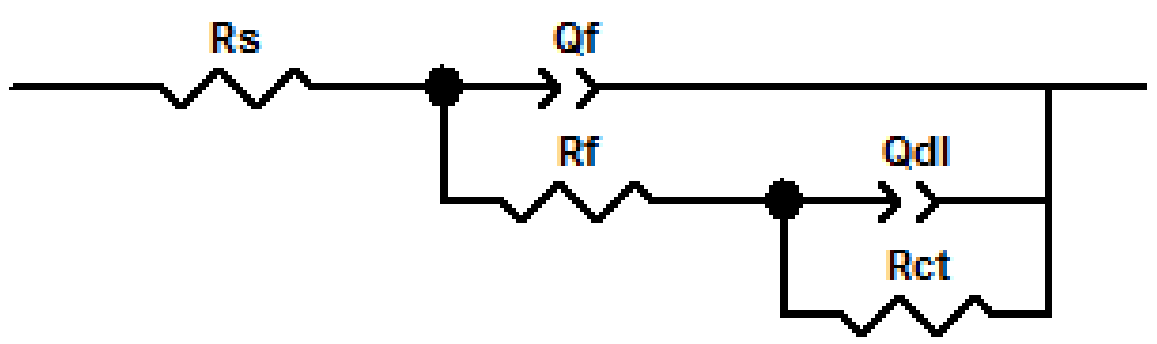

Figure 4-19 Equivalent circuit used to fit the EIS data

\subsubsection{Test results in morpholine solution}

Figure 4-20 and Figure 4-21 illustrate the Nyquist plots of Stellite 6 alloy and 17-4PH stainless steel tested in morpholine solution respectively. The Nyquist is presented in a complex plane, in which Z' and Z" represent the X-axis and Y-axis, respectively. The fitted values of parameters from the EIS curves are summarized in Table 4-4. The numbers in parentheses are fitting errors in units of the least significant digit. 


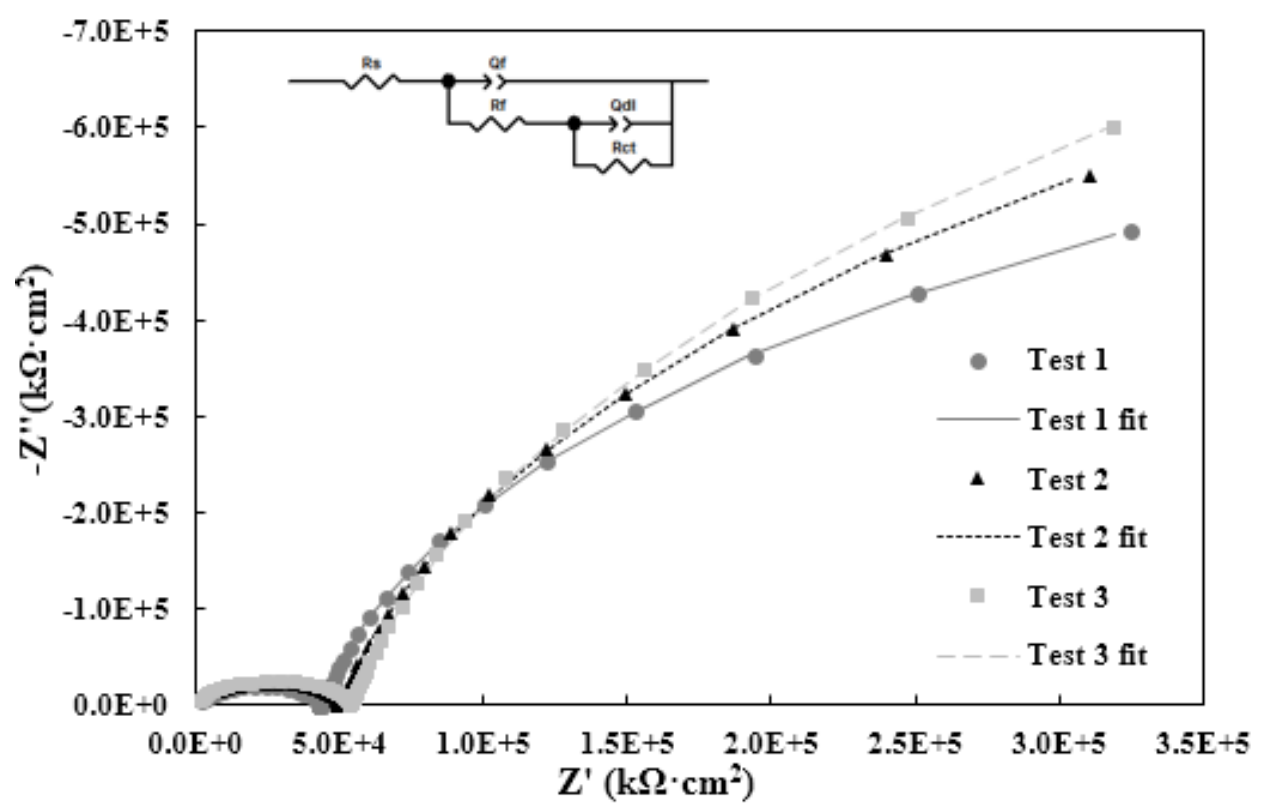

Figure 4-20 Nyquist plots of Stellite 6 alloy in morpholine solution

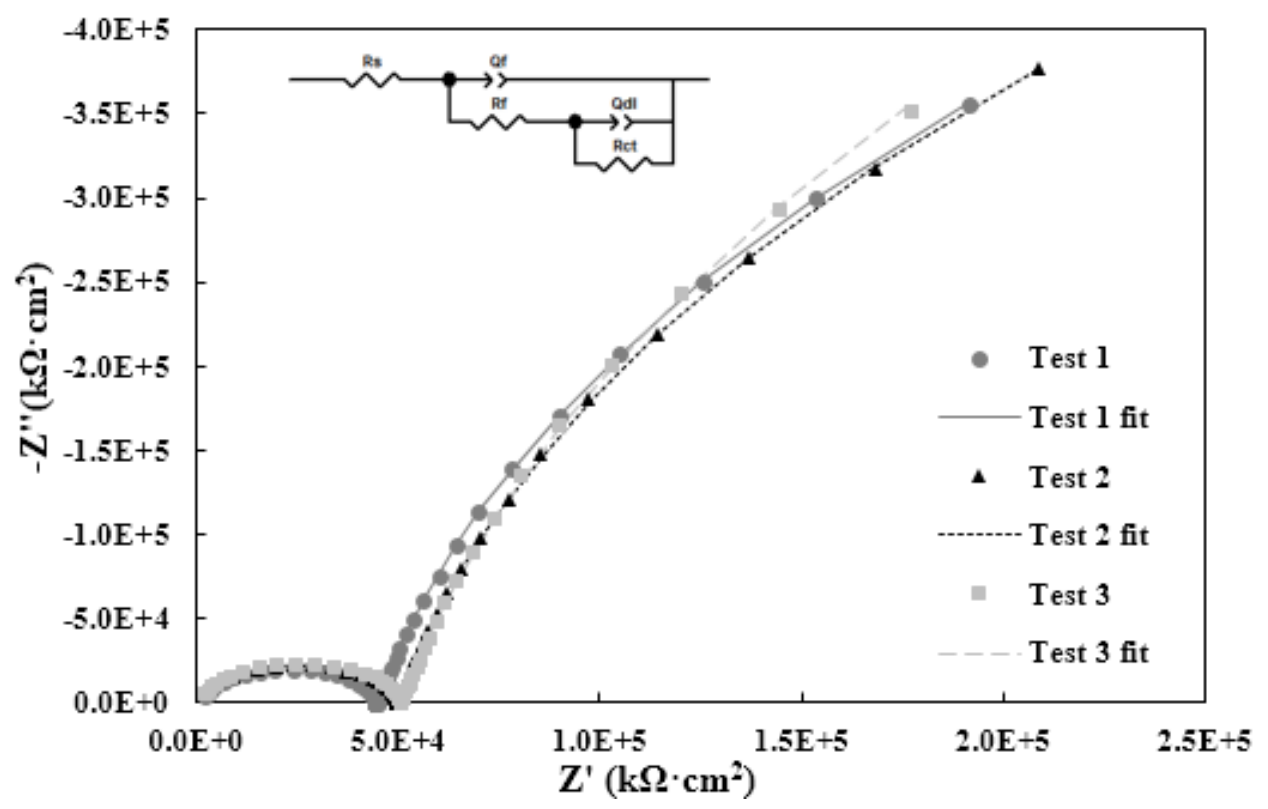

Figure 4-21 Nyquist plots of 17-4PH stainless steel in morpholine solution 
Table 4-4 Electrochemical corrosion parameters fitted from the equivalent circuit in morpholine solution

\begin{tabular}{|c|c|c|c|c|c|c|}
\hline \multirow{2}{*}{ Specimen } & \multicolumn{3}{|c|}{ Stellite 6 } & \multicolumn{3}{c|}{ 17-4PH } \\
\cline { 2 - 7 } & Test 1 & Test 2 & Test 3 & Test 1 & Test 2 & Test 3 \\
\hline $\begin{array}{c}\mathbf{R}_{\mathbf{s}} \\
\left(\mathbf{k} \boldsymbol{\Omega} \cdot \mathbf{c m}^{\mathbf{2}}\right)\end{array}$ & $1.52(3)$ & $1.46(2)$ & $1.39(2)$ & $1.50(2)$ & $1.48(2)$ & $1.58(2)$ \\
\hline $\begin{array}{c}\mathbf{Q}_{\mathbf{f}}, \mathbf{Y}_{\mathbf{0}} \times \mathbf{1 0}^{-\mathbf{1 1}} \\
\left(\boldsymbol{s}^{\boldsymbol{\alpha}} / \mathbf{\Omega c m}^{-\mathbf{2}}\right)\end{array}$ & $6.4(1)$ & $6.3(1)$ & $6.8(1)$ & $6.7(1)$ & $6.8(1)$ & $6.1(1)$ \\
\hline $\boldsymbol{\alpha}_{\mathbf{1}}$ & $0.954(2)$ & $0.955(1)$ & $0.950(1)$ & $0.952(1)$ & $0.950(1)$ & $0.957(1)$ \\
\hline $\begin{array}{c}\mathbf{R}_{\mathbf{f}} \\
\left(\mathbf{k} \boldsymbol{\Omega} \cdot \mathbf{c m}^{\mathbf{2}}\right)\end{array}$ & $41.81(4)$ & $48.51(5)$ & $52.62(5)$ & $43.06(4)$ & $48.23(4)$ & $49.21(5)$ \\
\hline $\begin{array}{c}\mathbf{Q}_{\mathbf{d l}}, \mathbf{Y}_{\mathbf{0}} \times \mathbf{1 0}^{-\mathbf{5}} \\
\left(\boldsymbol{s}^{\boldsymbol{\alpha}} / \mathbf{\Omega c m}^{-\mathbf{2}}\right)\end{array}$ & $2.007(4)$ & $1.928(4)$ & $1.809(4)$ & $2.957(7)$ & $2.792(6)$ & $3.085(8)$ \\
\hline $\mathbf{\alpha}_{\mathbf{2}}$ & $0.923(1)$ & $0.919(1)$ & $0.921(1)$ & $0.904(1)$ & $0.905(1)$ & $0.901(1)$ \\
\hline $\begin{array}{c}\mathbf{R}_{\mathbf{c t}} \\
\left(\mathbf{M} \boldsymbol{\Omega} \cdot \mathbf{c m}^{\mathbf{2}}\right)\end{array}$ & $1.46(2)$ & $1.98(3)$ & $2.27(3)$ & $1.61(3)$ & $1.64(3)$ & $2.00(5)$ \\
\hline
\end{tabular}

The difference in the EIS results between Stellite 6 alloy and the 17-4PH is not obvious. As seen from Table 4-4, the values of $R_{s}, Q_{f}, \alpha_{1,} R_{f}$ and $R_{c t}$ for the two materials are all very similar. The CPE $Q_{d l}$ of 17-4PH stainless steel is slightly larger than that of Stellite 6 alloy. Because the admittance is reversely proportional to the impedance, Stellite 6 alloy shows slightly better corrosion resistance than 17-4PH stainless steel in morpholine solution. The $\alpha_{2}$ value of Stellite 6 alloy is larger than that of $17-4 \mathrm{PH}$ stainless steel, which means that the oxide film formed on 17-4PH stainless steel is more fractal [96].

\subsubsection{Test results in cyclohexylamine solution}

Figure 4-22 and Figure 4-23 illustrate the Nyquist plots of Stellite 6 alloy and 17-4PH stainless steel tested in cyclohexylamine solution respectively. The fitted values of parameters from the EIS curves are summarized in Table 4-5. 


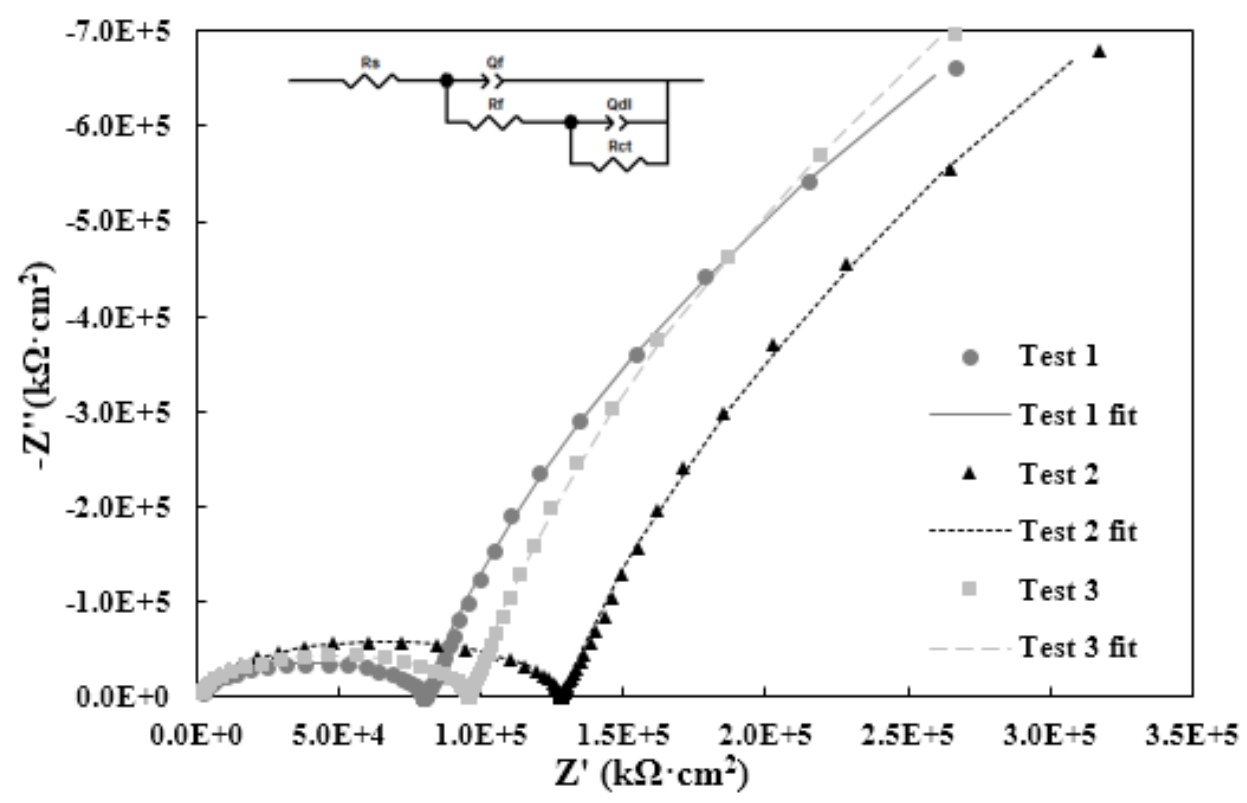

Figure 4-22 Nyquist plots of Stellite 6 alloy in cyclohexylamine solution

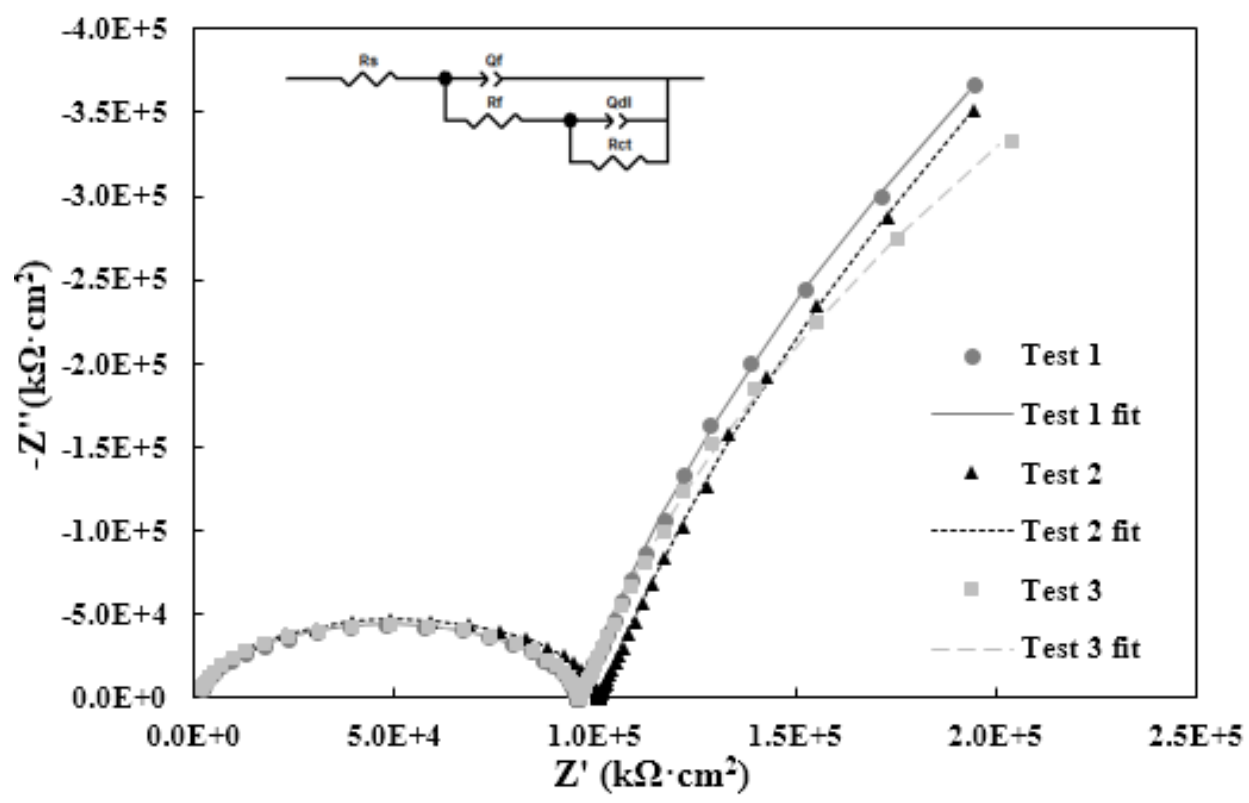

Figure 4-23 Nyquist plots of 17-4PH stainless steel in cyclohexylamine solution 
Table 4-5 Electrochemical corrosion parameters fitted from the equivalent circuit in cyclohexylamine solution

\begin{tabular}{|c|c|c|c|c|c|c|}
\hline \multirow{2}{*}{ Specimen } & \multicolumn{3}{|c|}{ Stellite 6 } & \multicolumn{3}{|c|}{ 17-4PH } \\
\cline { 2 - 7 } & Test 1 & Test 2 & Test 3 & Test 1 & Test 2 & Test 3 \\
\hline $\begin{array}{c}\mathbf{R}_{\mathbf{s}} \\
\left(\mathbf{k} \boldsymbol{\Omega} \cdot \mathbf{c m}^{\mathbf{2}}\right)\end{array}$ & $1.28(5)$ & $1.16(4)$ & $1.29(3)$ & $1.29(4)$ & $1.35(4)$ & $1.36(4)$ \\
\hline $\begin{array}{c}\mathbf{Q}_{\mathbf{f}}, \mathbf{Y}_{\mathbf{0}} \times \mathbf{1 0}^{-\mathbf{1 1}} \\
\left(\boldsymbol{s}^{\boldsymbol{\alpha}} / \mathbf{\Omega c m}^{-\mathbf{2}} \mathbf{)}\right.\end{array}$ & $6.5(2)$ & $6.3(1)$ & $6.2(1)$ & $5.8(1)$ & $5.3(1)$ & $5.7(1)$ \\
\hline $\mathbf{\alpha}_{\mathbf{1}}$ & $0.951(2)$ & $0.949(2)$ & $0.953(1)$ & $0.957(1)$ & $0.963(1)$ & $0.957(1)$ \\
\hline $\begin{array}{c}\mathbf{R}_{\mathbf{f}} \\
\left(\mathbf{k} \boldsymbol{\Omega} \cdot \mathbf{c m}^{\mathbf{2}}\right)\end{array}$ & $78.4(1)$ & $127.1(2)$ & $94.5(1)$ & $94.2(1)$ & $99.6(1)$ & $94.8(1)$ \\
\hline $\begin{array}{c}\mathbf{Q}_{\mathbf{d l}}, \mathbf{Y}_{\mathbf{0}} \times \mathbf{1 0}^{-\mathbf{5}} \\
\left(\boldsymbol{s}^{\boldsymbol{\alpha}} / \mathbf{\Omega c m}^{-\mathbf{2}}\right)\end{array}$ & $1.830(9)$ & $1.76(1)$ & $1.788(7)$ & $3.12(2)$ & $3.21(2)$ & $3.34(2)$ \\
\hline $\mathbf{\alpha}_{\mathbf{2}}$ & $0.920(3)$ & $0.914(3)$ & $0.927(2)$ & $0.897(3)$ & $0.891(4)$ & $0.898(3)$ \\
\hline $\begin{array}{c}\mathbf{R}_{\mathbf{c t}} \\
\left(\mathbf{M} \boldsymbol{\Omega} \cdot \mathbf{c m}^{\mathbf{2}}\right)\end{array}$ & $4.7(3)$ & $5.5(4)$ & $5.9(3)$ & $3.7(3)$ & $4.0(5)$ & $2.4(2)$ \\
\hline
\end{tabular}

As seen from Table 4-5, the values of $R_{s}, Q_{f}, \alpha_{1}$ and $R_{f}$ for the two materials are very close, but the CPE $Q_{d l}$ of $17-4 \mathrm{PH}$ stainless steel is larger than that of Stellite 6 alloy, and the charge transfer resistance $R_{c t}$ of $17-4 \mathrm{PH}$ stainless steel is evidently smaller than that of Stellite 6 alloy. Therefore, Stellite 6 alloy has much better corrosion resistance than $17-4 \mathrm{PH}$ stainless steel in cyclohexylamine solution. The $\alpha_{2}$ values indicate that the oxide film formed in cyclohexylamine solution is similar to that in morpholine solution.

\subsubsection{Test results in sodium hydroxide solution}

Figure 4-24 and Figure 4-25 illustrate the Nyquist plots of Stellite 6 alloy and 17-4PH stainless steel tested in sodium hydroxide solution respectively. The fitted values of parameters from the EIS curves are summarized in Table 4-6. The values of $R_{s}, Q_{f}, \alpha_{1}, R_{f}$ and $\alpha_{2}$ for the two materials are very similar, but the CPE $Q_{d l}$ of $17-4 \mathrm{PH}$ stainless steel is larger than that of Stellite 6 alloy, and the charge transfer resistance $R_{c t}$ of $17-4 \mathrm{PH}$ stainless 
steel is apparently smaller than that of Stellite 6 alloy. Therefore, Stellite 6 alloy has better corrosion resistance than $17-4 \mathrm{PH}$ stainless steel in sodium hydroxide solution.

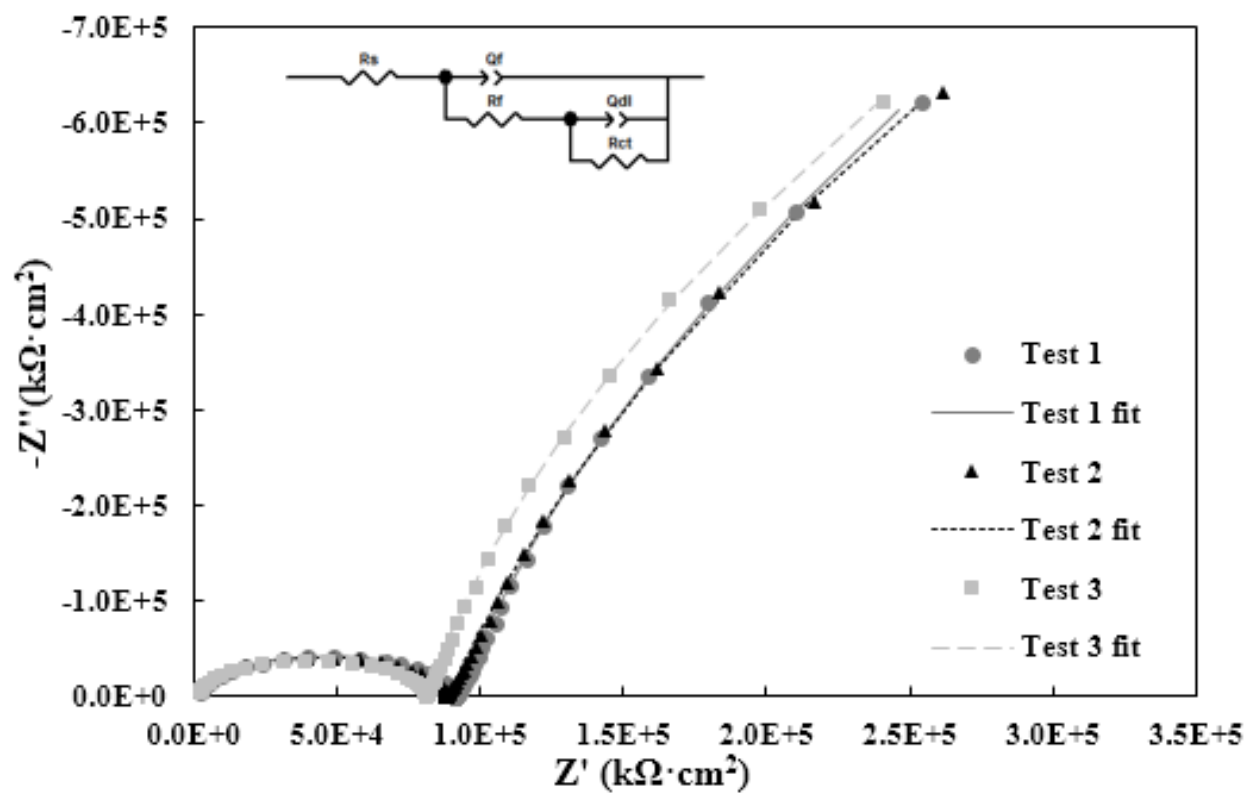

Figure 4-24 Nyquist plots of Stellite 6 alloy in sodium hydroxide solution

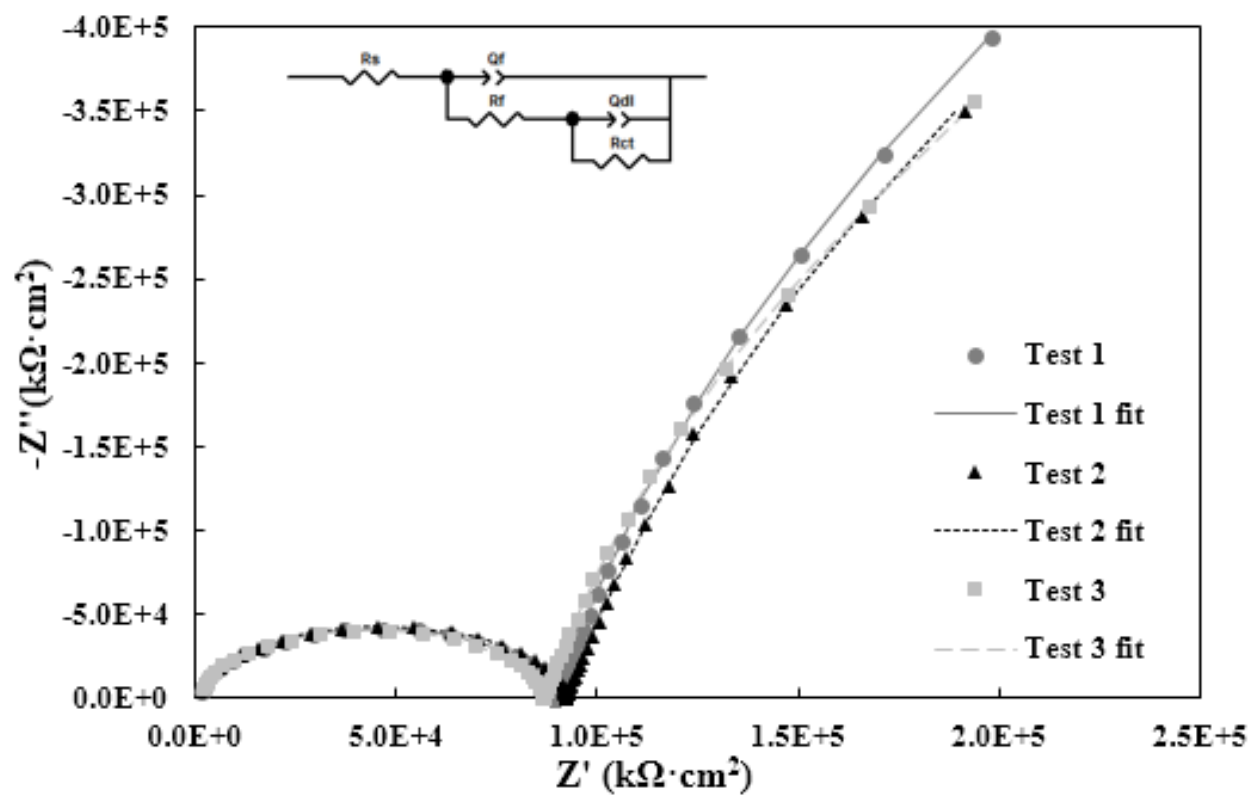

Figure 4-25 Nyquist plots of 17-4PH stainless steel in sodium hydroxide solution 
Table 4-6 Electrochemical corrosion parameters fitted from the equivalent circuit in sodium hydroxide solution

\begin{tabular}{|c|c|c|c|c|c|c|}
\hline \multirow{2}{*}{ Specimen } & \multicolumn{3}{|c|}{ Stellite 6 } & \multicolumn{3}{c|}{ 17-4PH } \\
\cline { 2 - 7 } & Test 1 & Test 2 & Test 3 & Test 1 & Test 2 & Test 3 \\
\hline $\begin{array}{c}\mathbf{R}_{\mathbf{s}} \\
\left(\mathbf{k} \boldsymbol{\Omega} \cdot \mathbf{c m}^{\mathbf{2}}\right)\end{array}$ & $1.45(5)$ & $1.43(4)$ & $1.43(4)$ & $1.34(4)$ & $1.30(3)$ & $1.48(4)$ \\
\hline $\begin{array}{c}\mathbf{Q}_{\mathbf{f}}, \mathbf{Y}_{\mathbf{0}} \times \mathbf{1 0}^{-11} \\
\left(\boldsymbol{s}^{\boldsymbol{\alpha}} / \mathbf{\Omega}^{-\mathbf{2}}\right)\end{array}$ & $5.3(2)$ & $4.8(1)$ & $5.1(1)$ & $5.6(1)$ & $5.7(1)$ & $5.1(1)$ \\
\hline $\boldsymbol{\alpha}_{\mathbf{1}}$ & $0.963(2)$ & $0.971(2)$ & $0.967(2)$ & $0.962(2)$ & $0.963(2)$ & $0.967(2)$ \\
\hline $\begin{array}{c}\mathbf{R}_{\mathbf{f}} \\
\left(\mathbf{k} \boldsymbol{\Omega} \cdot \mathbf{c m}^{\mathbf{2}}\right)\end{array}$ & $89.7(2)$ & $87.0(1)$ & $79.9(1)$ & $88.2(1)$ & $91.0(1)$ & $85.3(1)$ \\
\hline $\begin{array}{c}\mathbf{Q}_{\mathbf{d l}}, \mathbf{Y}_{\mathbf{0}} \times \mathbf{1 0}^{-\mathbf{5}} \\
\left(\boldsymbol{s}^{\boldsymbol{\alpha}} / \mathbf{\Omega c m}^{-\mathbf{2}}\right)\end{array}$ & $1.20(1)$ & $1.846(9)$ & $1.985(8)$ & $2.95(2)$ & $3.28(2)$ & $3.21(2)$ \\
\hline $\boldsymbol{\alpha}_{\mathbf{2}}$ & $0.909(3)$ & $0.903(3)$ & $0.926(2)$ & $0.908(3)$ & $0.903(3)$ & $0.906(3)$ \\
\hline $\begin{array}{c}\mathbf{R}_{\mathbf{c t}} \\
\left(\mathbf{M} \boldsymbol{\Omega} \cdot \mathbf{c m}^{\mathbf{2}}\right)\end{array}$ & $6.0(6)$ & $6.0(5)$ & $4.8(3)$ & $3.3(2)$ & $3.1(2)$ & $2.5(2)$ \\
\hline
\end{tabular}




\section{Chapter 5: Failure Tests and Analyses}

Since no obvious damage was found on the samples after the normal electromechanical tests, failure test was performed on Stellite 6 alloy and 17-4PH stainless steel. The objectives of this test were four: for each alloy (1) to find a means to fail the sample surface in morpholine solution; (2) to use this means as a standard method to fail the sample surface in cyclohexylamine solution and sodium hydroxide solution at the same $\mathrm{pH}$; (3) to make comparison in the polarization curves between Stellite 6 alloy and 17-4PH stainless steel in the three solutions; (4) to obtain the morphologies of the failed surfaces.

\subsection{Failure test method}

Although the experimental studies in Chapter 4 reveal the different corrosion properties of Stellite 6 alloy and 17-4PH stainless steel in the three solutions, since EIS method is known to be a nondestructive test method and the sample surfaces were not significantly changed after the polarization tests, no obvious damage was observed on the sample surfaces using these test methods.

Stellite 6 alloy showed better corrosion resistances than 17-4PH stainless steel in both amine solutions (morpholine solution and cyclohexylamine) and sodium hydroxide solution under the same $\mathrm{pH}$ condition. However, it was suggested that Stellite 6 alloy might be replaced by stainless steels such as $17-4 \mathrm{PH}$ stainless steel in amine related environments [4]. One question remained unsolved if there any possibility that Stellite 6 would have better corrosion resistance than $17-4 \mathrm{PH}$ stainless steel in the less corrosive environment, 
while 17-4PH stainless steel would have better corrosion resistance than Stellite 6 alloy when corrosion became severe. Therefore, it was necessary to find a way to fail the sample of Stellite 6 alloy and understand how it failed in the solutions. If Stellite 6 alloy can be failed in the testing condition, there is possibility that the hardfacings of Stellite 6 alloy could fail in the same failure mechanism. If there was visual surface change (color change due to formation of corrosion products) or the solution was no longer transparent, it was assumed that damage or failure of the tested material occurred.

One test was performed on a used sample of Stellite 6 alloy in morpholine solution. The aim of this test was to find if a higher potential could make the sample fail in morpholine solution. Therefore, OCP test was not run on this sample. As was demonstrated in Figure $3-19$, the scan rate, initial and end potential remained unchanged, except that the highest voltage was set to be $10 \mathrm{~V}$. However, the forward scan cannot go up to the set value and it stopped at a potential around 3.6 V. To avoid damaging the corrosion cell and the Solartron; the scan was reversed manually. The obtained polarization curve is shown in Figure 5-1. The corrosion product on the sample can be observed, see Figure 5-2. The camera was positioned pointing at the testing sample, as shown in Figure 3-17 (b).

The reason why the scan wandered at a certain level of voltage is still unknown, however, this phenomenon happened again in more similar parallel tests. This problem only occurred when a scan rate of $0.1667 \mathrm{mV} / \mathrm{s}$ was used. When the scan rate was set to be $1 \mathrm{mV} / \mathrm{s}$, $5 \mathrm{mV} / \mathrm{s}$, and $10 \mathrm{mV} / \mathrm{s}$, there were no hesitations for the scan to reach the set value of voltage. 


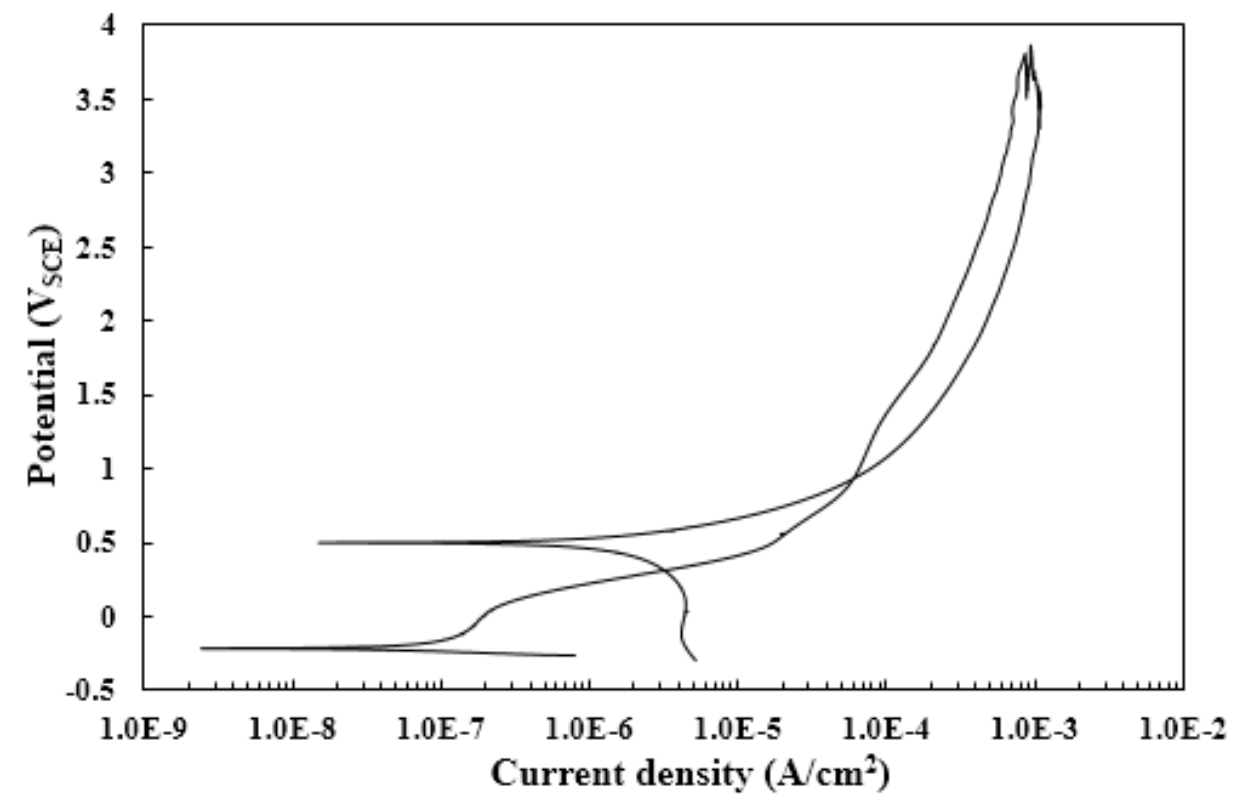

Figure 5-1 Cyclic polarization curve of Stellite 6 alloy tested in morpholine solution at high potentials

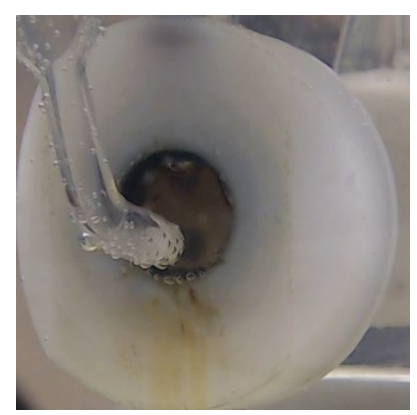

Figure 5-2 Photo of the Stellite 6 alloy sample tested in morpholine solution for cyclic polarization at high potentials

Based on this observation, an assumption could be made that Stellite 6 alloy could be damaged at a higher voltage over a certain period. It seemed that $4 \mathrm{~V}$ was high enough for Stellite 6 to fail. Potentiostatic polarization method works by supplying a constant potential over a certain period of time, and the current can be monitored at the same time. Therefore, potentiostatic polarization method was chosen for the failure tests. 
However, it was hard to determine if all the samples had reached a same final state of corrosion failure. It was more realistic to set a same potential and a same time for all the samples to corrode in the three corrosive media. If the samples were corroded in one solution, but not in the others, it would mean that the first solution is more corrosive for the sample.

Since $4 \mathrm{~V}$ potential was high enough for the sample to become corroded under cyclic polarization, the potential was set to be $4 \mathrm{~V}$ in the failure tests. Trials were performed first in morpholine solution. In the first trial for Stellite 6 alloy, the time range was set to be 70000 s. It was found that the sample started to corrode after $25000 \mathrm{~s}$, which resulted in the corrosion product formed on the sample surface, as shown in Figure 5-3 (a). The corrosion was even more severe at the end of the potentiostatic polarization test, as demonstrated by more corrosion product on the sample surface, shown in Figure 5-3 (b). The SEM/EDX results of the collected corrosion products were recorded in Appendix.

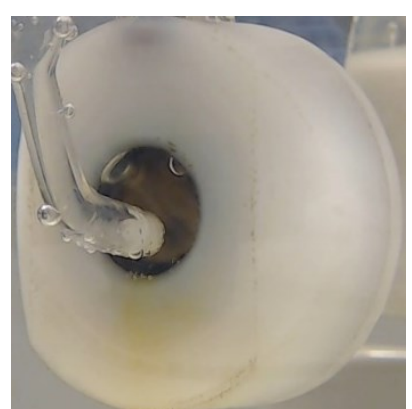

(a)

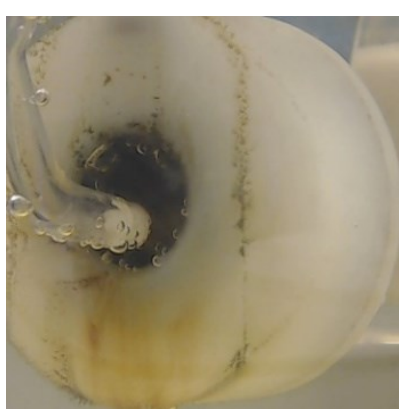

(b)

Figure 5-3 Photo of the Stellite 6 alloy sample tested in morpholine solution under potentiostatic polarization: (a) for $25000 \mathrm{~s}$; (b) for $70000 \mathrm{~s}$ 
In the first trial for $17-4 \mathrm{PH}$ stainless steel, the time range was set to be $10000 \mathrm{~s}$. It was found that the sample started to corrode after $1500 \mathrm{~s}$, which was characterized by the corrosion product on the sample surface, shown in Figure 5-4 (a). However, the polarization tests must be stopped manually at the time of $2550 \mathrm{~s}$, because the tip of the bridge tube was only about $2 \mathrm{~mm}$ away from the sample surface and the corrosion product of the sample surface may block the porous tip of the bridge tube. The sample surface with corrosion product after the polarization for $2550 \mathrm{~s}$ is shown in Figure 5-4 (b).

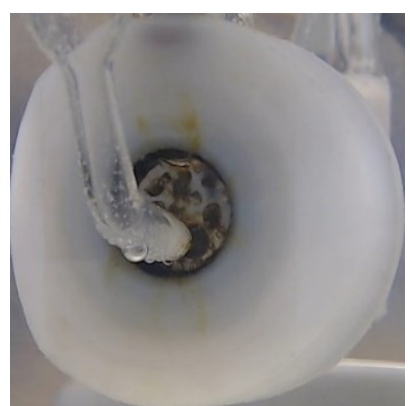

(a)

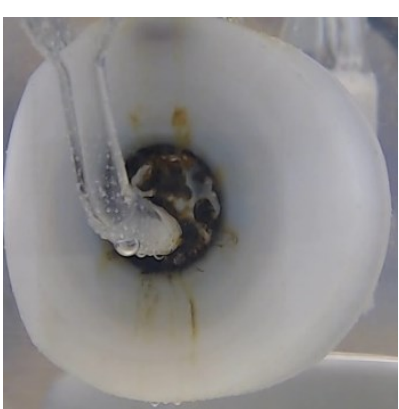

(b)

Figure 5-4 Photo of the 17-4PH stainless steel sample tested in morpholine solution under potentiostatic polarization: (a) for $1500 \mathrm{~s}$; (b) for $2550 \mathrm{~s}$

\subsection{Failure tests of Stellite 6 alloy}

\subsubsection{Experimental setup}

It was found in the trial test of potentiostatic polarization that the Stellite 6 alloy sample could fail in morpholine solution when $4 \mathrm{~V}$ constant potential was applied to the system after $25000 \mathrm{~s}$ and corrosion product was found on the sample surface. According to the trial test results, the failure test setup for Stellite 6 alloy was decided as detailed in Figure $5-5$. 


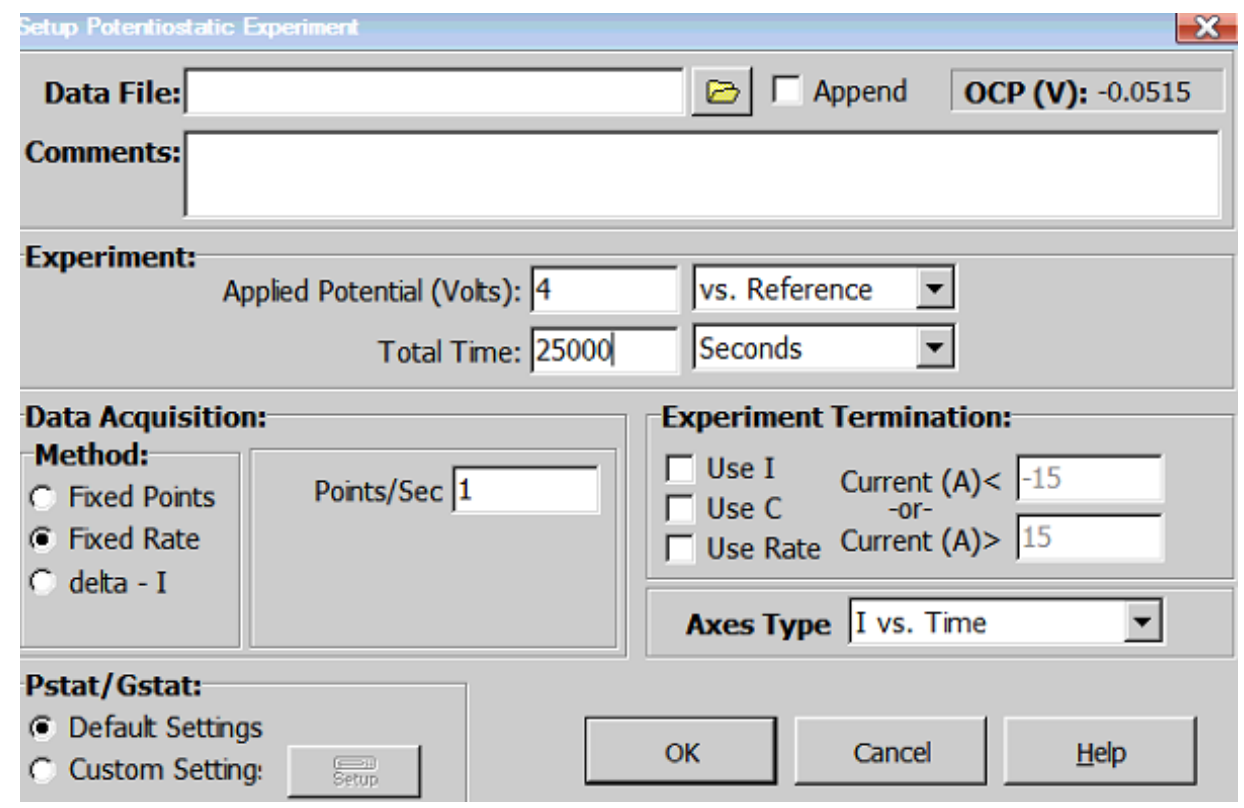

Figure 5-5 Potentiostatic polarization failure test setup for Stellite 6 alloy

\subsubsection{Failure tests in morpholine solution}

\subsubsection{Polarization test results}

To monitor the surface changing process of Stellite 6 alloy in morpholine solution under potentiostatic polarization, the pictures were taken at every $5000 \mathrm{~s}$ from the start to the end of the test, which are given in Figure 5-6 in time sequence. The corresponding polarization curve of $I$ vs. time is presented in Figure 5-7. 


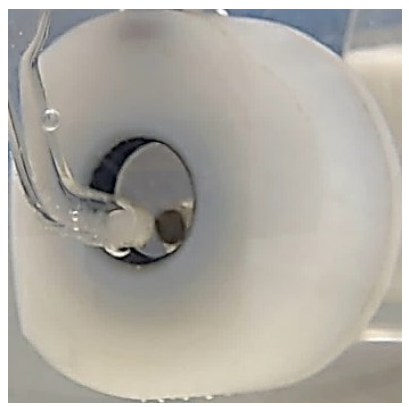

(a)

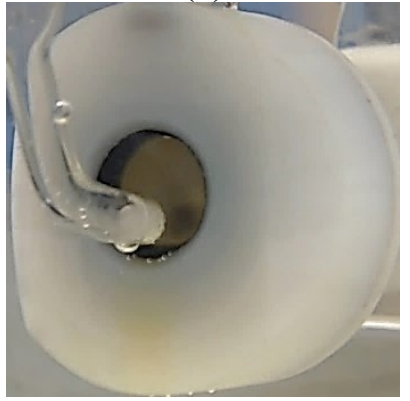

(d)

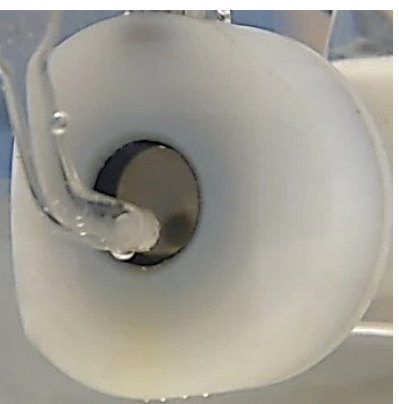

(b)

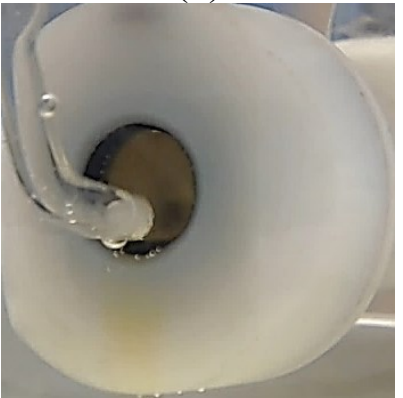

(e)

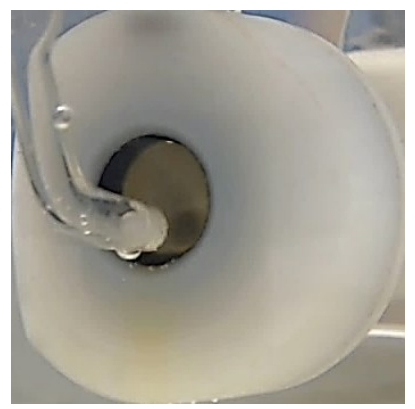

(c)

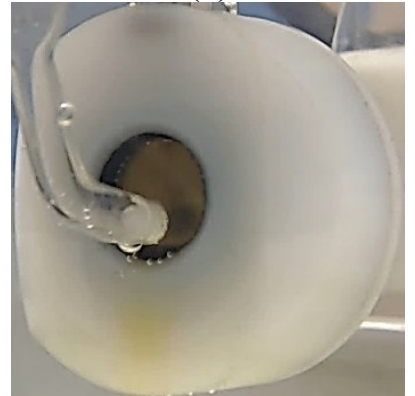

(f)

Figure 5-6 Photos of the Stellite 6 alloy sample tested in morpholine solution under potentiostatic polarization: (a) for $0 \mathrm{~s}$; (b) for $5000 \mathrm{~s}$; (c) for $10000 \mathrm{~s}$; (d) for $15000 \mathrm{~s}$; (e) for $20000 \mathrm{~s}$; (f) for $25000 \mathrm{~s}$

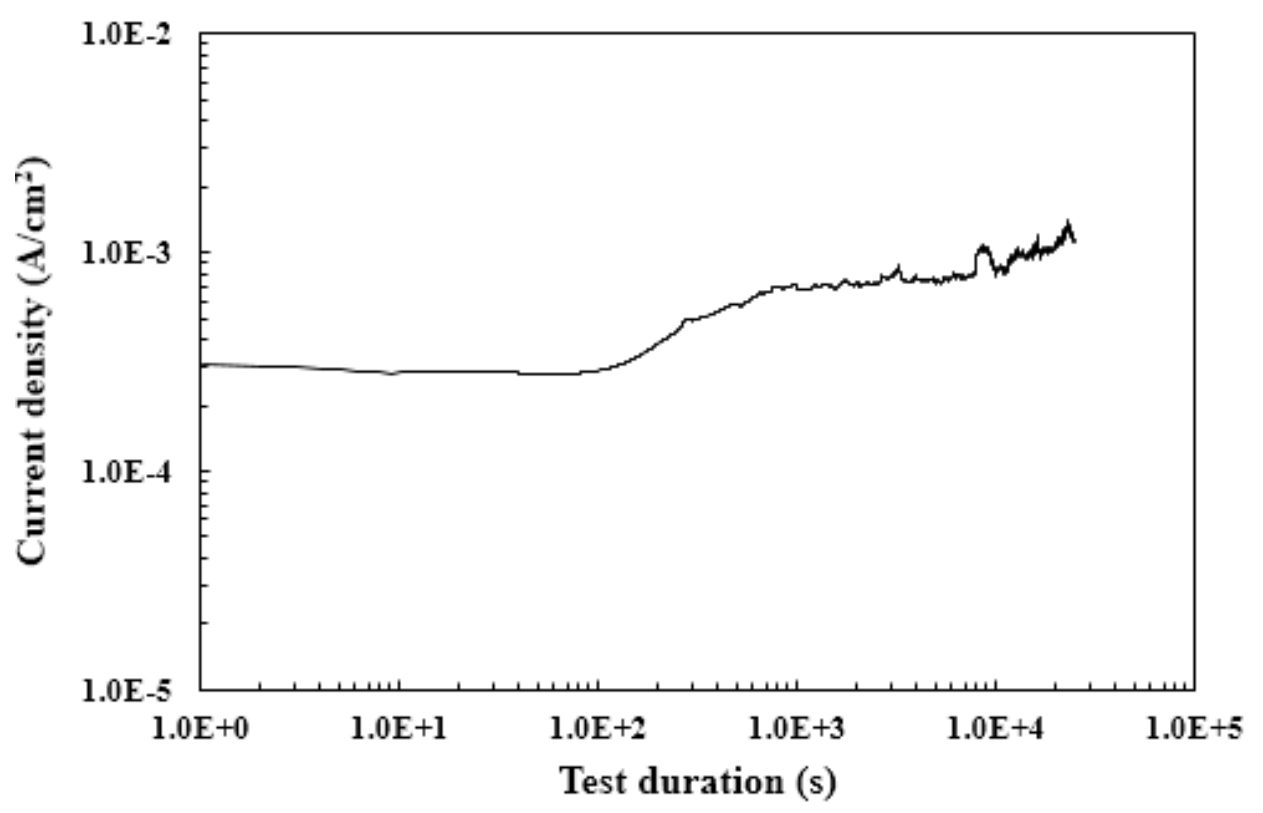

Figure 5-7 Variation of current with time of the Stellite 6 alloy sample tested in morpholine solution under potentiostatic polarization 
The sample surface turned dark gradually when corrosion occurred, as shown in Figure 5-6. This phenomenon indicated that the corrosion was becoming more severe as time increased, which was consistent with the current curve in Figure 5-7. The current gradually decreased at the initial stage and had a minimum at the time about $100 \mathrm{~s}$ and then increased rapidly. Finally, it experienced a relatively smooth increasing. This was because once potential was applied, the corrosion process caused an oxide film formed on the sample surface, resulting in decreasing of the current. However, when the corrosion proceeded, the oxide film broke, and the base material was then attacked by the corrosive medium, leading to the increase of the current. The corrosion product left on the sample surface was the result of the spallation of the oxide film, whose amount increased with the corrosion progress.

\subsubsection{Analyses of corroded surfaces}

To further understand the failure mechanisms of the material in the corrosive medium, the corroded surfaces of the Stellite 6 alloy samples tested in morpholine solution were analyzed using SEM. The surface morphology images are shown in Figure 5-8. Compared with the intact surface in Figure 3-2, the corroded surface of Stellite 6 is fully covered with cracked oxides. To investigate the oxides of the sample surface, EDX spectroscopy system was utilized to analyze the chemical constituents of the oxides, and the results are reported in Figure 5-9 to Figure 5-11. Due to the deficient capability of the EDX instrument in quantifying accurately $\mathrm{C}$ and $\mathrm{O}$ contents, the information provided in the elemental content table can only be used for qualitative analysis, but not for quantitative analysis. 


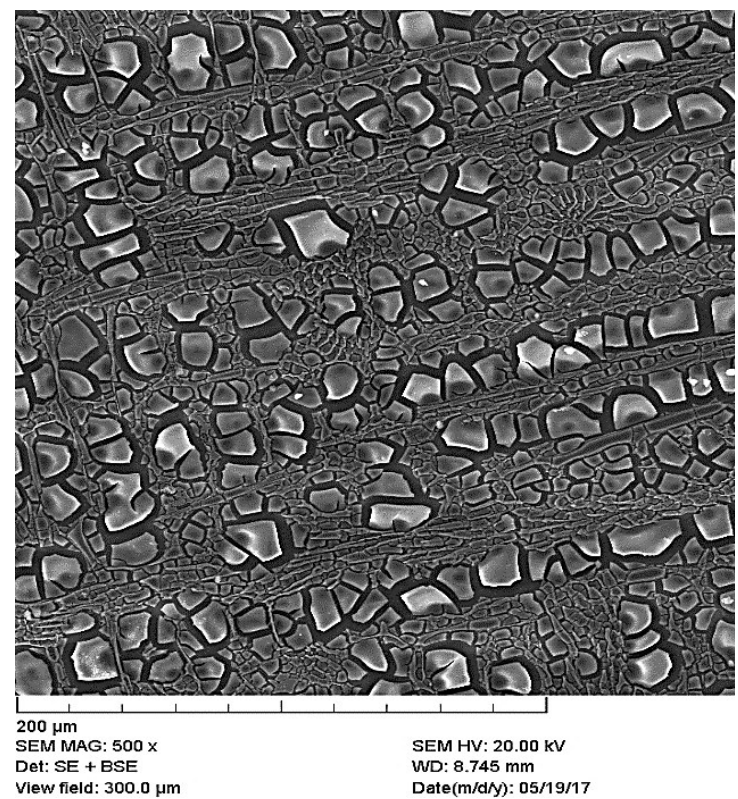

(a)

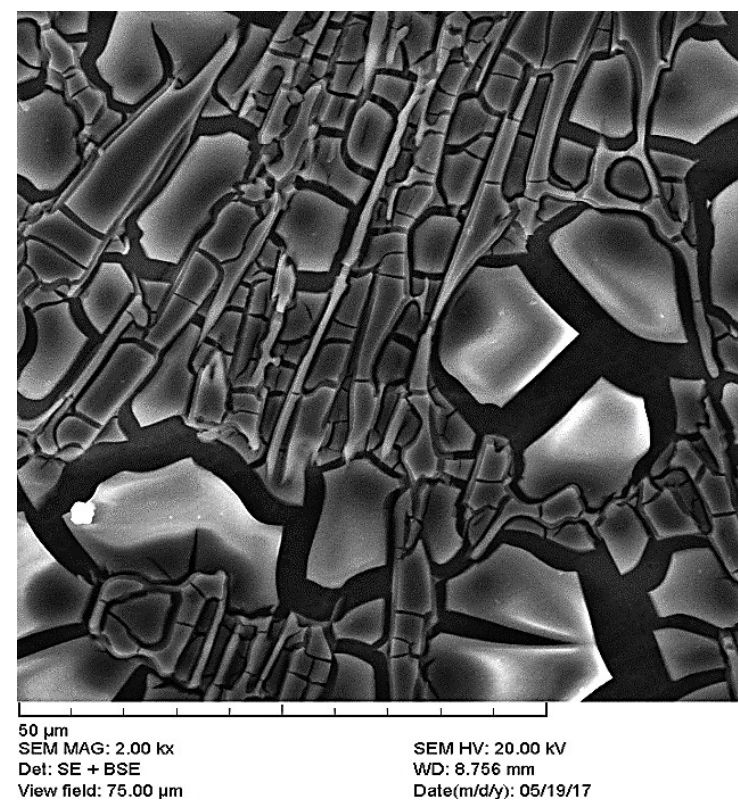

(b)

Figure 5-8 SEM images of corroded surface of the Stellite 6 alloy sample tested in morpholine solution: (a) at low magnification; (b) at high magnification

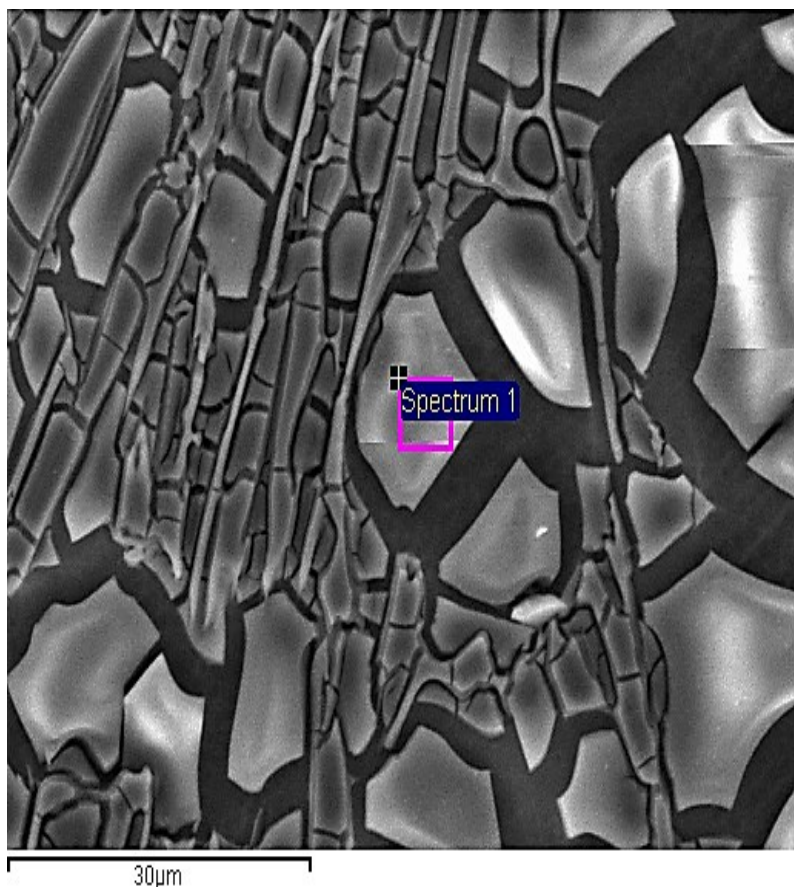

(a)

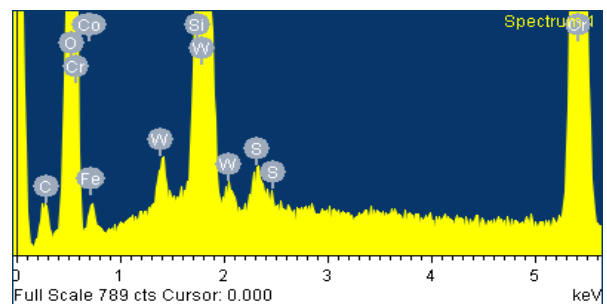

(b)

\begin{tabular}{|c|c|c|}
\hline Element & $\mathbf{w t} \%$ & at\% \\
\hline C K & 4.86 & 8.47 \\
\hline O K & 49.86 & 65.24 \\
\hline Si K & 2.26 & 1.69 \\
\hline S K & 0.61 & 0.40 \\
\hline Cr K & 34.52 & 13.90 \\
\hline Fe K & 11.59 & 4.35 \\
\hline Co K & 4.92 & 1.75 \\
\hline W M & 37.00 & 4.21 \\
\hline Totals & 145.62 & 100.00 \\
\hline
\end{tabular}

(c)

Figure 5-9 EDX results of the white area on the corroded surface of the Stellite 6 alloy sample tested in morpholine solution: (a) SEM morphology; (b) EDX spectrum; (c) elemental content table 


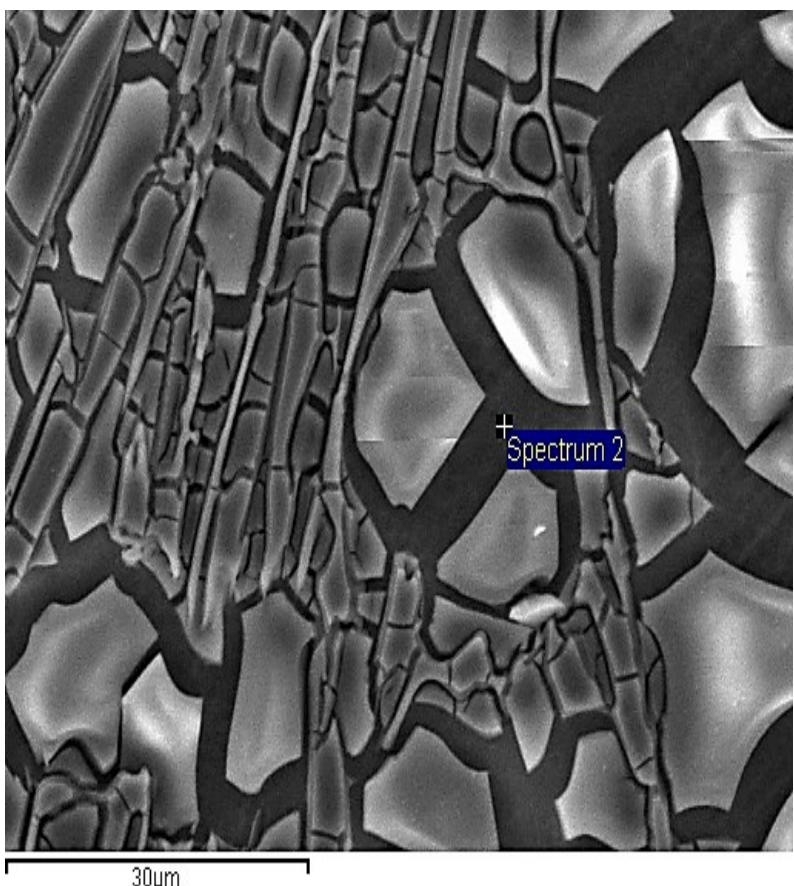

(a)

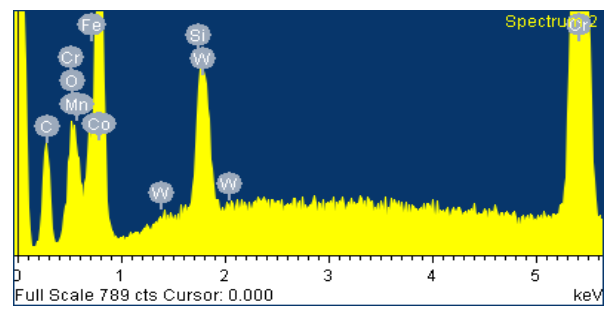

(b)

\begin{tabular}{|c|c|c|}
\hline Element & wt\% & at\% \\
\hline C K & 16.64 & 33.98 \\
\hline O K & 2.75 & 4.21 \\
\hline Si K & 1.24 & 1.09 \\
\hline Cr K & 38.20 & 18.02 \\
\hline Mn K & 1.36 & 0.61 \\
\hline Fe K & 2.94 & 1.29 \\
\hline Co K & 95.88 & 39.90 \\
\hline W M & 6.88 & 0.92 \\
\hline Totals & 165.89 & 100.00 \\
\hline
\end{tabular}

(c)

Figure 5-10 EDX results of the black area on the corroded surface of the Stellite 6 alloy sample tested in morpholine solution: (a) SEM morphology; (b) EDX spectrum; (c) elemental content table

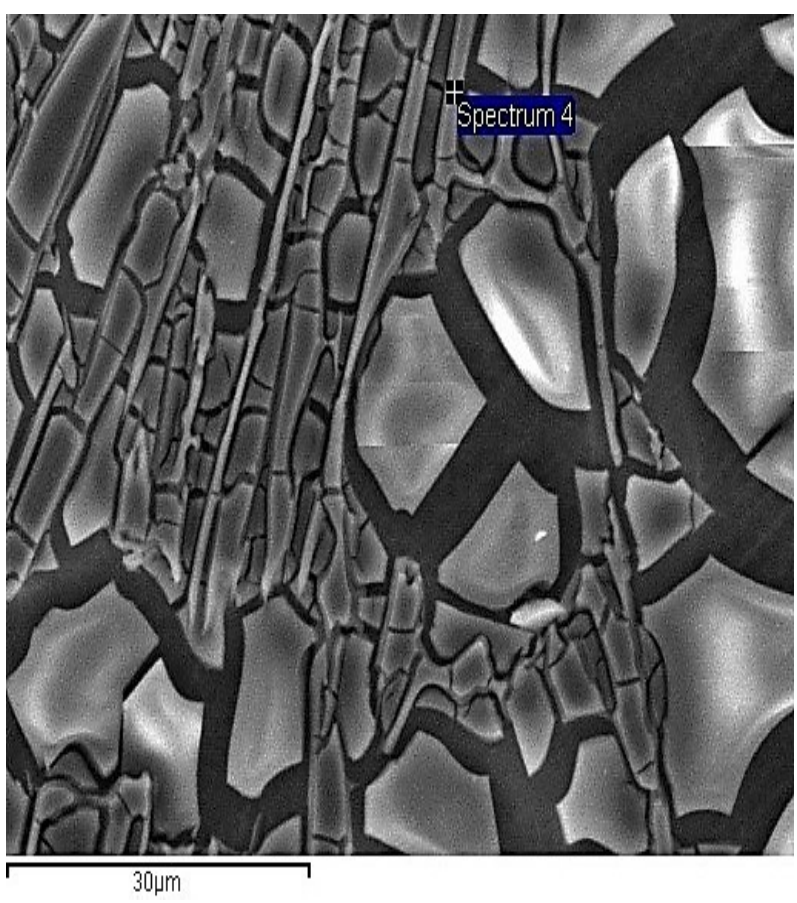

(a)

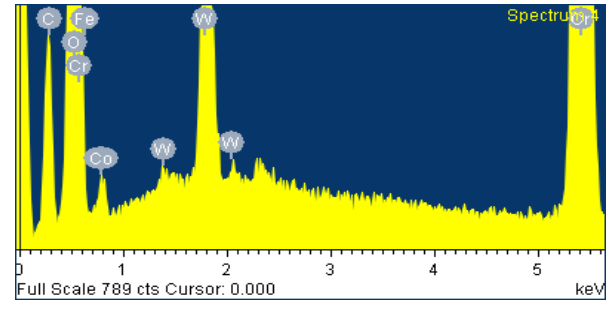

(b)

\begin{tabular}{|c|c|c|}
\hline Element & $\mathbf{w t} \%$ & $\mathbf{a t} \%$ \\
\hline C K & 22.01 & 25.95 \\
\hline O K & 49.03 & 43.38 \\
\hline Cr K & 93.81 & 25.54 \\
\hline Fe K & 1.77 & 0.45 \\
\hline Co K & 14.51 & 3.49 \\
\hline W M & 15.52 & 1.19 \\
\hline Totals & 196.65 & 100.00 \\
\hline
\end{tabular}

(c)

Figure 5-11 EDX results of the grey area on the corroded surface of the Stellite 6 alloy sample tested in morpholine solution: (a) SEM morphology; (b) EDX spectrum; (c) elemental content table 
Three distinct areas, in white, black and grey, respectively, of the corroded surface were analyzed using EDX. The white area contains very high $\mathrm{O}$ and $\mathrm{Cr}$ so that it must be the Cr-rich oxide. There are also high Fe and $\mathrm{W}$ contents, which indicate the presence of Fe-rich and W-rich oxides. For the black area, because of the low $\mathrm{O}$ contents but the high $\mathrm{C}, \mathrm{Cr}$, and Co contents, it should be the substrate, which is exposed out due to the spallation of the oxides. The grey area is found to have very high $\mathrm{O}$ and $\mathrm{Cr}$ contents and high $\mathrm{C}$ content as well, therefore, Cr-rich oxides must be presented in this area. The high $\mathrm{C}$ content may come from the corrosion product of the carbides in Stellite 6 alloy.

\subsubsection{Failure tests in cyclohexylamine solution}

\subsubsection{Polarization test results}

Similar to the failure tests in morpholine solution, for monitoring the surface changing process of Stellite 6 alloy in cyclohexylamine solution under potentiostatic polarization, the pictures were taken at every $5000 \mathrm{~s}$ from the start to the end of the test. The obtained photos are presented in Figure 5-12 in time sequence. The corresponding polarization curve is presented in Figure 5-13. 


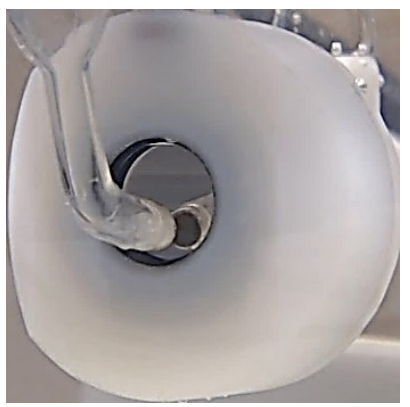

(a)

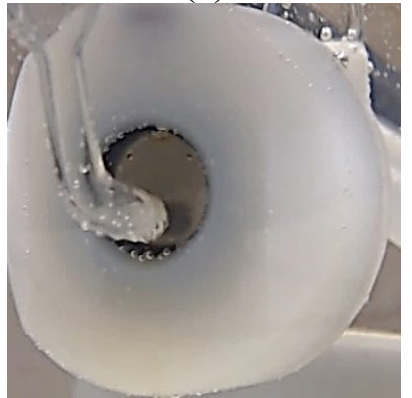

(d)

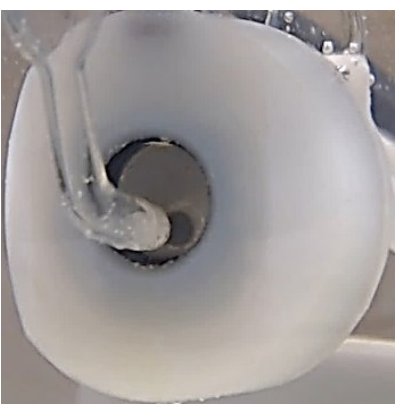

(b)

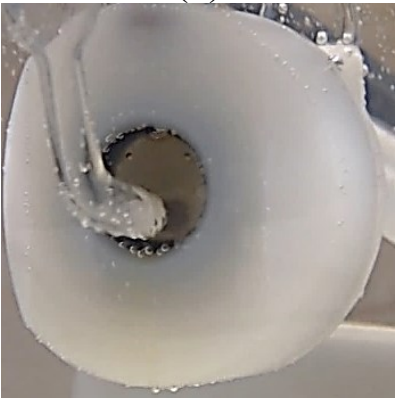

(e)

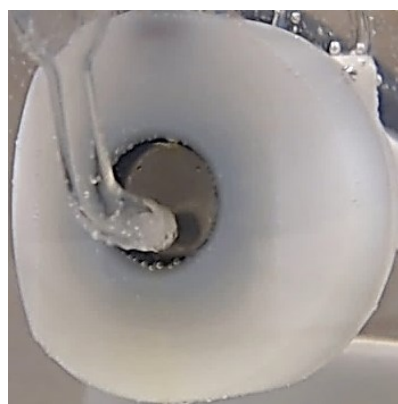

(c)

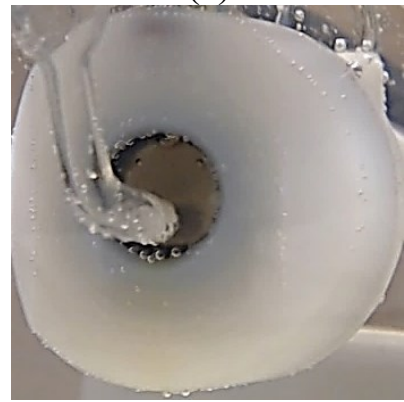

(f)

Figure 5-12 Photos of the Stellite 6 alloy sample tested in cyclohexylamine solution under potentiostatic polarization: (a) for $0 \mathrm{~s}$; (b) for $5000 \mathrm{~s}$; (c) for $10000 \mathrm{~s}$;

(d) for $15000 \mathrm{~s}$; (e) for $20000 \mathrm{~s}$; (f) for $25000 \mathrm{~s}$

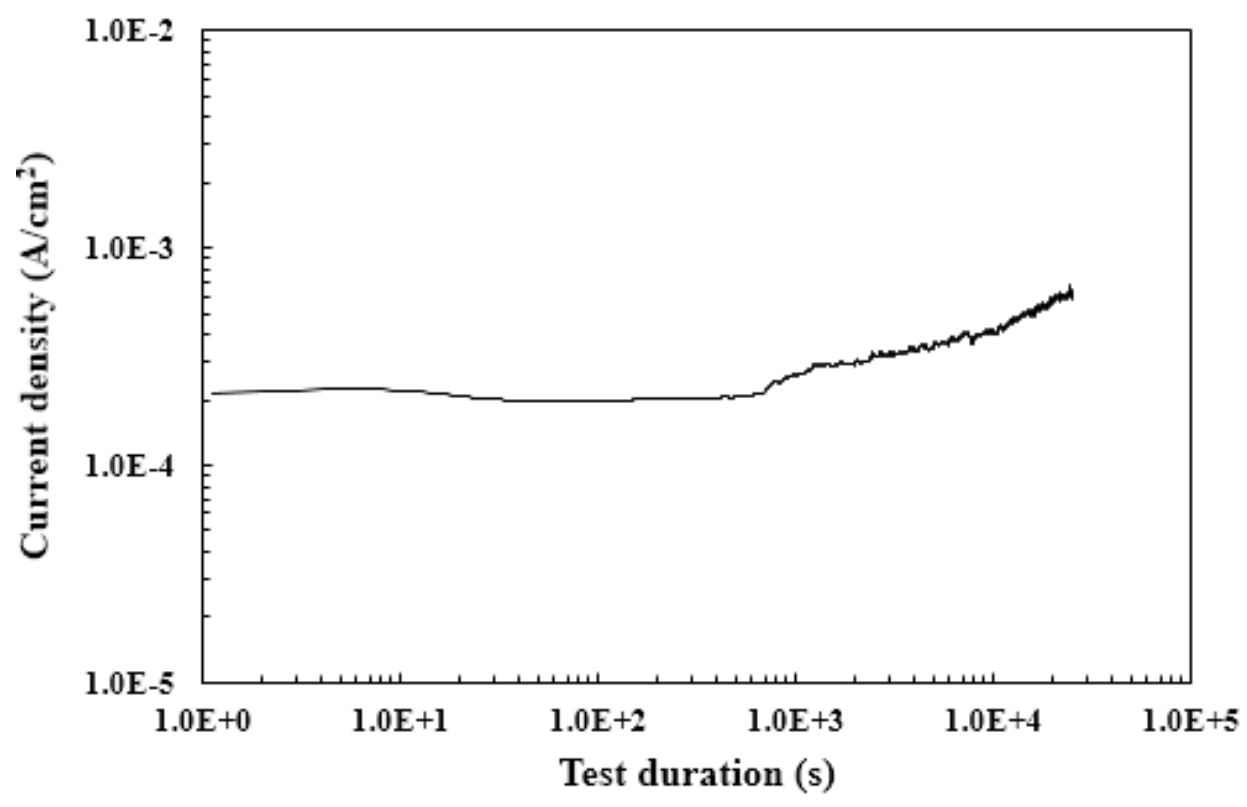

Figure 5-13 Variation of current with time of the Stellite 6 alloy sample tested in cyclohexylamine solution under potentiostatic polarization 
Similar to the test in morpholine solution, the sample surface also turned dark gradually in cyclohexylamine solution, as seen in Figure 5-12, indicating corrosion proceeded with time. For the current curve in Figure 5-13, the general trend of the curve is similar to that of the curve for morpholine solution. Compared with the current curve for the test in morpholine solution, the one for the test in cyclohexylamine solution has two main differences. First, at the initial stage, the current fluctuated and then consistently decreased to a minimum at the time about $20 \mathrm{~s}$. Second, this minimum was kept for a period of time about $900 \mathrm{~s}$, and then gradually increased. These imply that the formation process of the oxide film on Stellite 6 alloy in cyclohexylamine solution was slower but the oxide film was more electron-transferring resistant or had more protective effect.

\subsubsection{Analyses of corroded surfaces}

The corroded surfaces of the Stellite 6 alloy samples tested in cyclohexylamine solution were analyzed using SEM. The surface morphology images are shown in Figure 5-14. The surface is fully covered with cracked oxides. To further investigate the oxides of the sample surface, EDX spectroscopy system was utilized to analyze the chemical constituents of the oxides, and the results are reported in Figure 5-15 to Figure 5-17. 


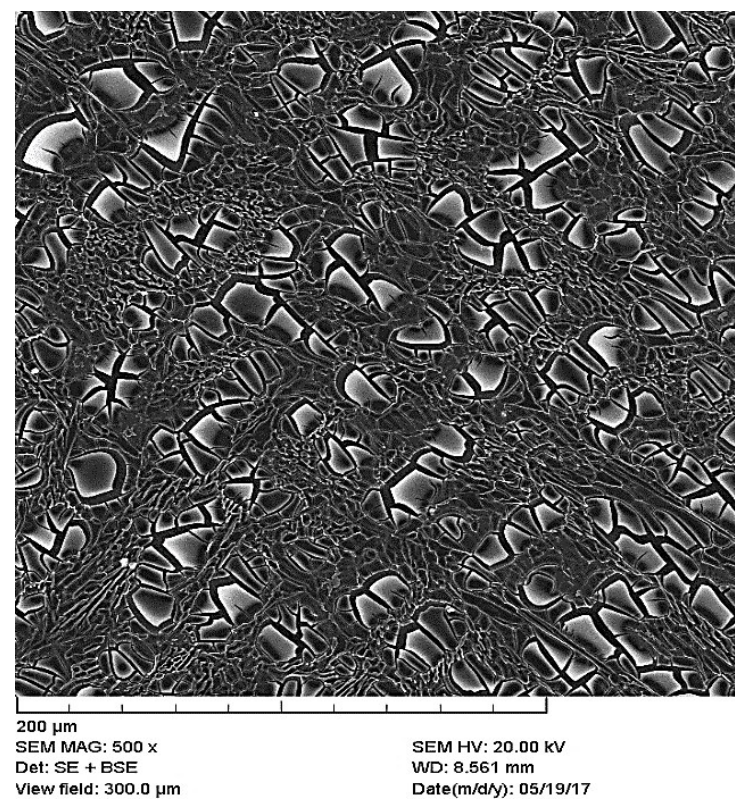

(a)

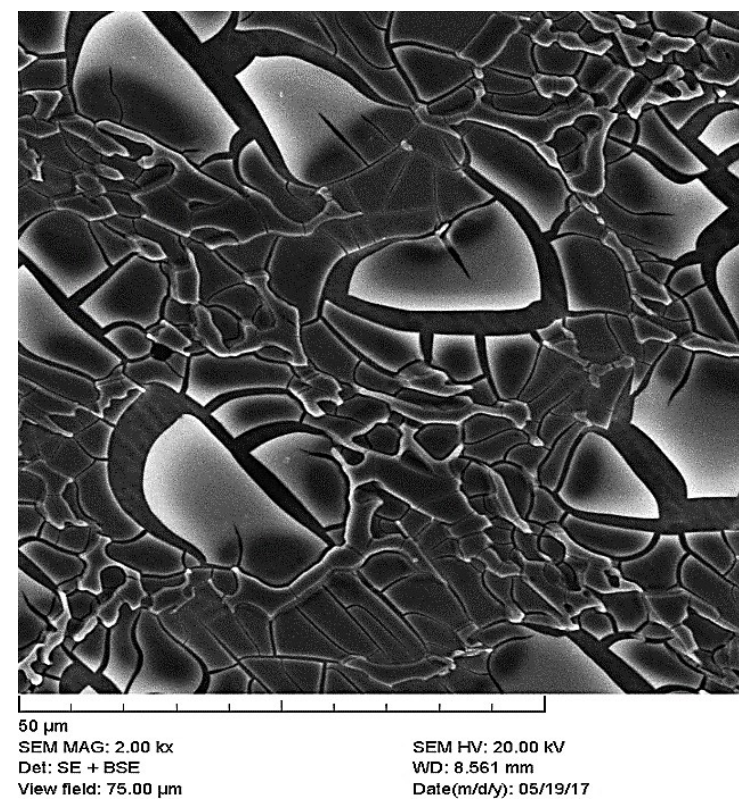

(b)

Figure 5-14 SEM images of corroded surface of the Stellite 6 alloy sample tested in cyclohexylamine solution: (a) at low magnification; (b) at high magnification

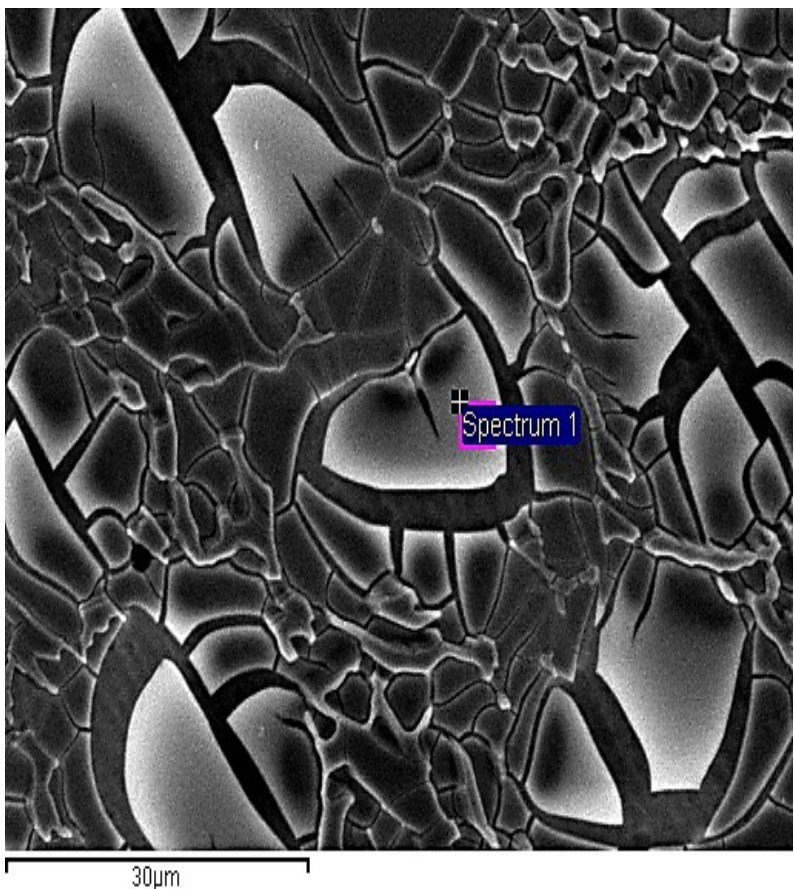

(a)

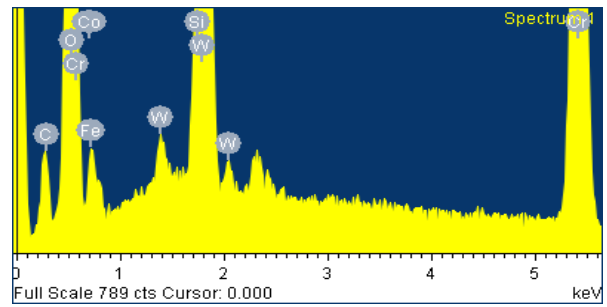

(b)

\begin{tabular}{|c|c|c|}
\hline Element & wt\% & at\% \\
\hline C K & 9.91 & 11.70 \\
\hline O K & 73.41 & 65.06 \\
\hline Si K & 2.96 & 1.49 \\
\hline Cr K & 37.68 & 10.28 \\
\hline Fe K & 11.26 & 2.86 \\
\hline Co K & 23.49 & 5.65 \\
\hline W M & 38.31 & 2.95 \\
\hline Totals & 197.02 & 100.00 \\
\hline
\end{tabular}

(c)

Figure 5-15 EDX results of the white area on the corroded surface of the Stellite 6 alloy sample tested in cyclohexylamine solution: (a) SEM morphology; (b) EDX spectrum; (c) elemental content table 


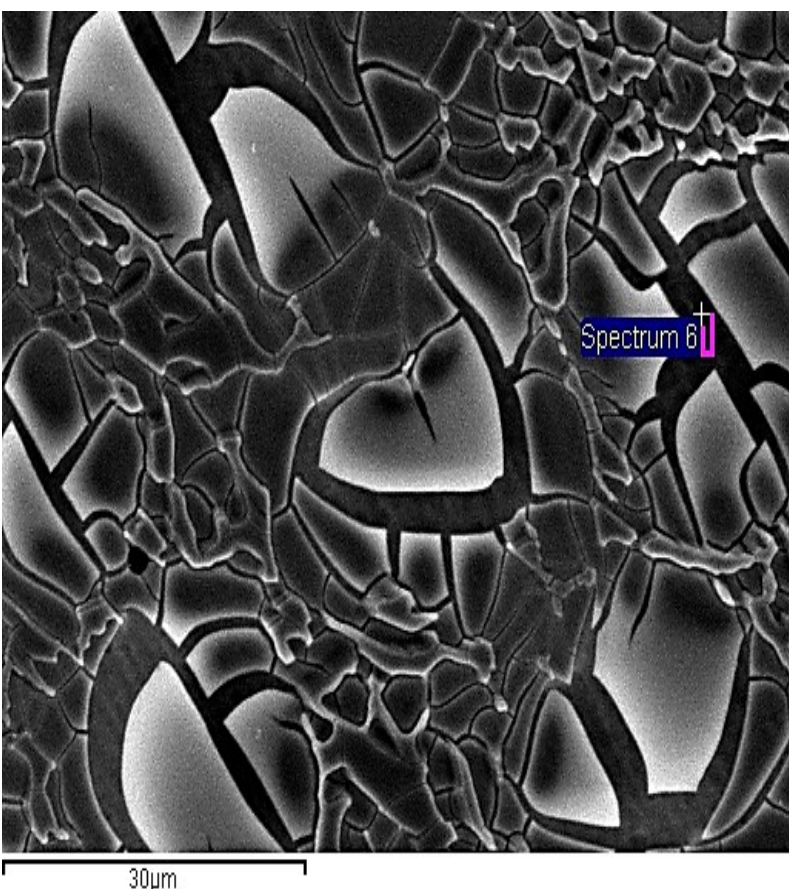

(a)

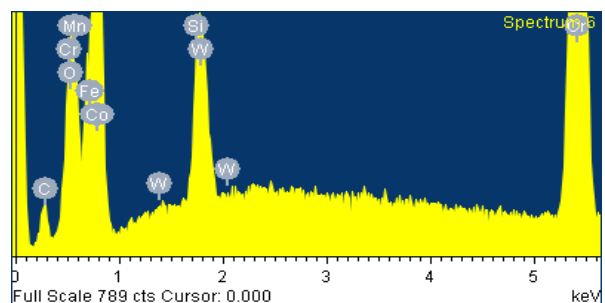

(b)

\begin{tabular}{|c|c|c|}
\hline Element & wt\% & at\% \\
\hline C K & 6.41 & 15.94 \\
\hline O K & 3.50 & 6.54 \\
\hline Si K & 1.93 & 2.05 \\
\hline Cr K & 39.92 & 22.92 \\
\hline Mn K & 1.35 & 0.73 \\
\hline Fe K & 3.21 & 1.72 \\
\hline Co K & 96.52 & 48.90 \\
\hline W M & 7.42 & 1.20 \\
\hline Totals & 160.26 & 100.00 \\
\hline \multicolumn{2}{|l}{}
\end{tabular}

(c)

Figure 5-16 EDX results of the black area on the corroded surface of the Stellite 6 alloy sample tested in cyclohexylamine solution: (a) SEM morphology; (b) EDX spectrum; (c) elemental content table

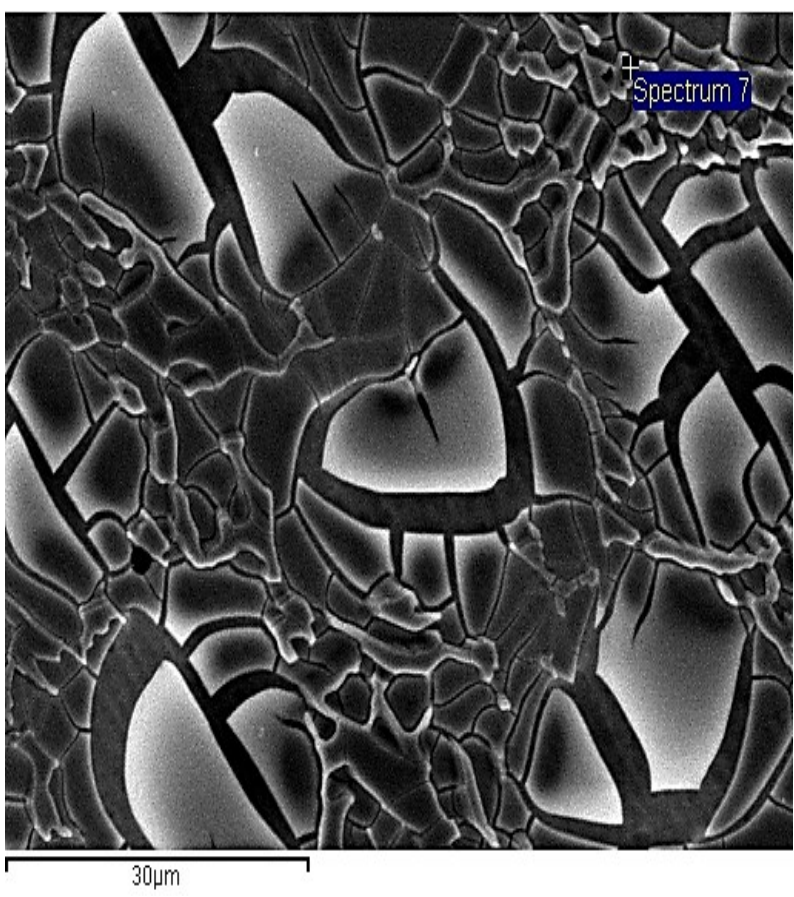

(a)

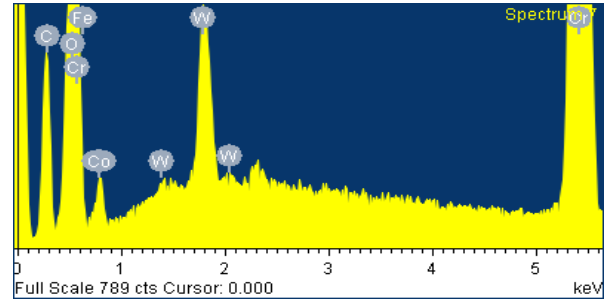

(b)

\begin{tabular}{|c|c|c|}
\hline Element & $\mathbf{w t} \%$ & $\mathbf{a t} \%$ \\
\hline C K & 22.58 & 32.29 \\
\hline O K & 23.14 & 24.84 \\
\hline Cr K & 109.08 & 36.02 \\
\hline Fe K & 1.27 & 0.39 \\
\hline Co K & 19.26 & 5.61 \\
\hline W M & 9.11 & 0.85 \\
\hline Totals & 184.43 & 100.00 \\
\hline
\end{tabular}

(c)

Figure 5-17 EDX results of the grey area on the corroded surface of the Stellite 6 alloy sample tested in cyclohexylamine solution: (a) SEM morphology; (b) EDX spectrum; (c) elemental content table 
As can be seen, the surface morphology of the Stellite 6 alloy sample tested in cyclohexylamine is similar to that of the sample tested in morpholine solution. There are three distinct areas, in white, black and grey, respectively, on the corroded surface, which were analyzed using EDX. Since the chemical compositions of the three areas are similar to the test in morpholine solution, the white area is mainly $\mathrm{Cr}$-rich oxide, the black area is the Stellite 6 substrate, and the grey area is Cr-rich oxides and the corrosion product of Cr-rich carbides.

\subsubsection{Failure tests in sodium hydroxide solution}

\subsubsection{Polarization test results}

Pictures were taken at every $5000 \mathrm{~s}$ from the start to the end of the test to monitor the surface changing process of Stellite 6 alloy in sodium hydroxide solution under potentiostatic polarization, as shown in Figure 5-18 in time sequence. The corresponding polarization curve is presented in Figure 5-19. 


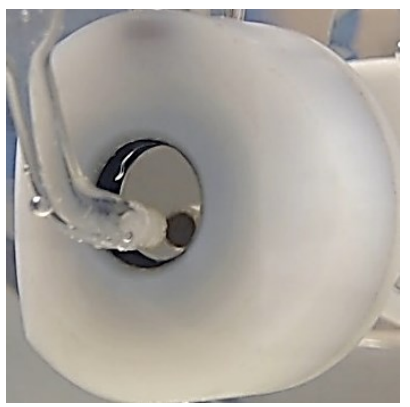

(a)

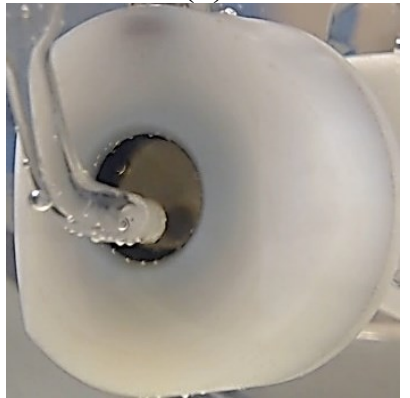

(d)

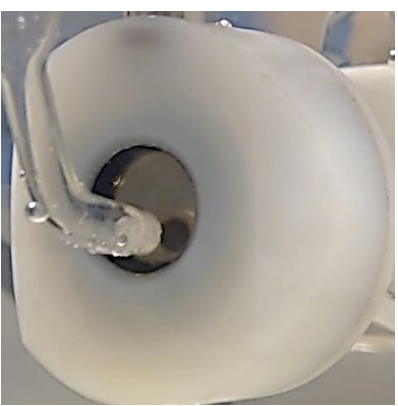

(b)

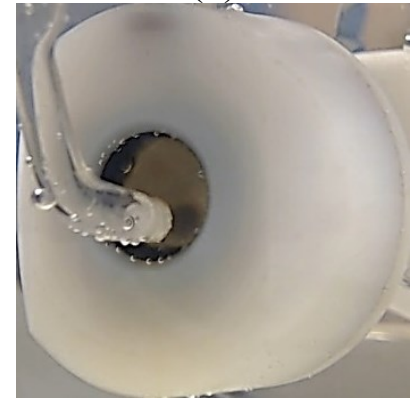

(e)

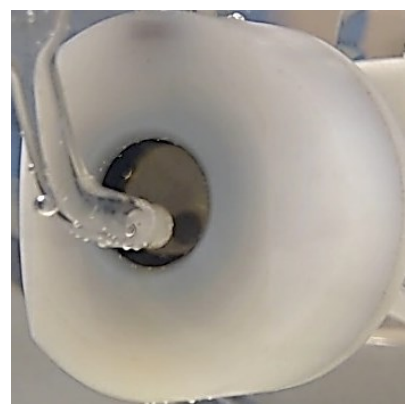

(c)

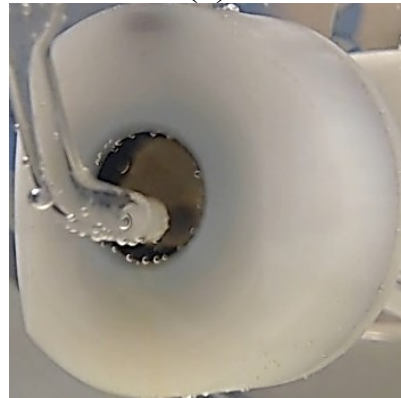

(f)

Figure 5-18 Photos of the Stellite 6 alloy sample tested in sodium hydroxide solution under potentiostatic polarization: (a) for $0 \mathrm{~s}$; (b) for $5000 \mathrm{~s}$; (c) for $10000 \mathrm{~s}$;

(d) for $15000 \mathrm{~s}$; (e) for $20000 \mathrm{~s}$; (f) for $25000 \mathrm{~s}$

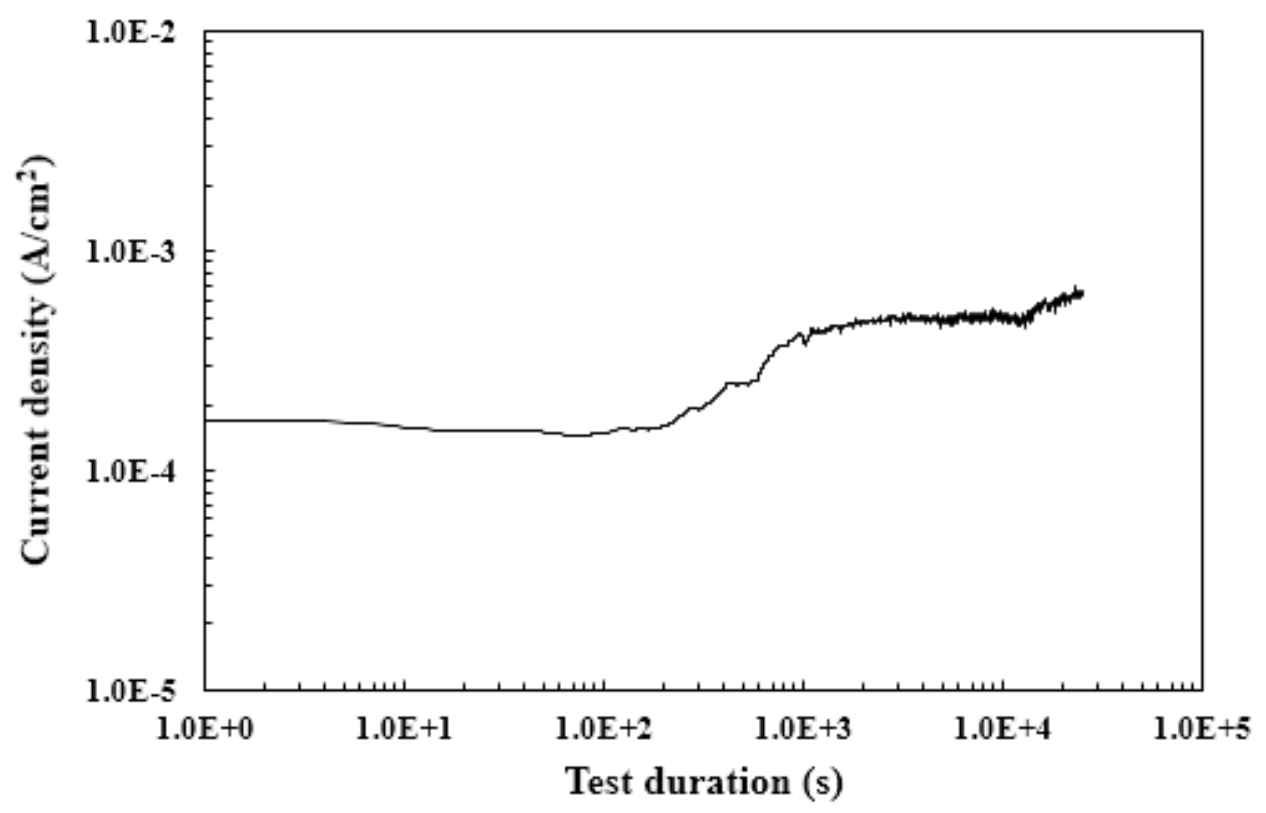

Figure 5-19 Variation of current with time of the Stellite 6 alloy sample tested in sodium hydroxide solution under potentiostatic polarization

The sample surface turned dark in sodium hydroxide solution due to corrosion, as shown 
in Figure 5-18, but the surface was not so severely corroded as in morpholine solution and cyclohexylamine solution. From the current density curve, in Figure 5-19, the continuous decrease of the current meant the steady formation of the oxide film on the sample surface during the failure test, leading to a minimum of the current at the time about $100 \mathrm{~s}$, and then the current increased rapidly, indicating the break of the oxide film.

\subsubsection{Analyses of corroded surfaces}

The corroded surfaces of the Stellite 6 alloy samples tested in sodium hydroxide solution were analyzed using SEM. The surface morphology images are shown in Figure 5-20. As seen, the surface is fully covered with cracked oxides. To further investigate the oxides of the sample surface, EDX spectroscopy system was utilized to analyze the chemical constituents of the oxides, and the results are reported in Figure 5-21 to Figure 5-23.

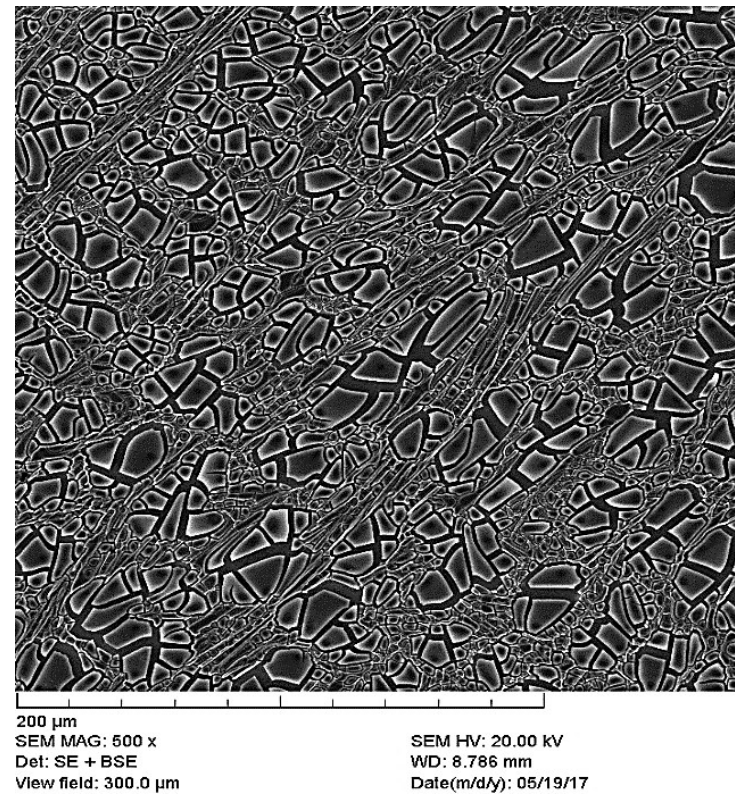

(a)

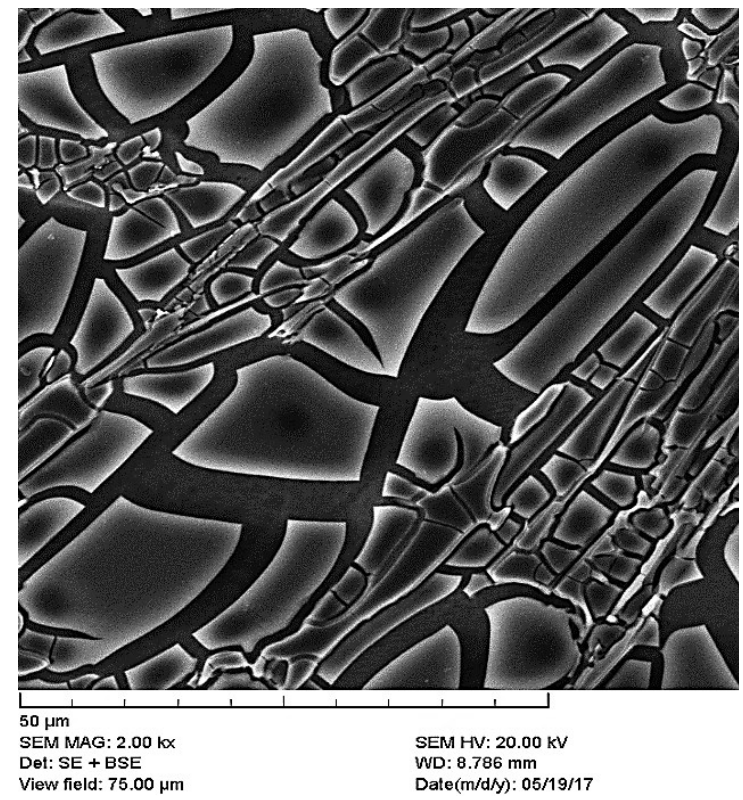

(b)

Figure 5-20 SEM images of corroded surface of the Stellite 6 alloy sample tested in sodium hydroxide solution: (a) at low magnification; (b) at high magnification 


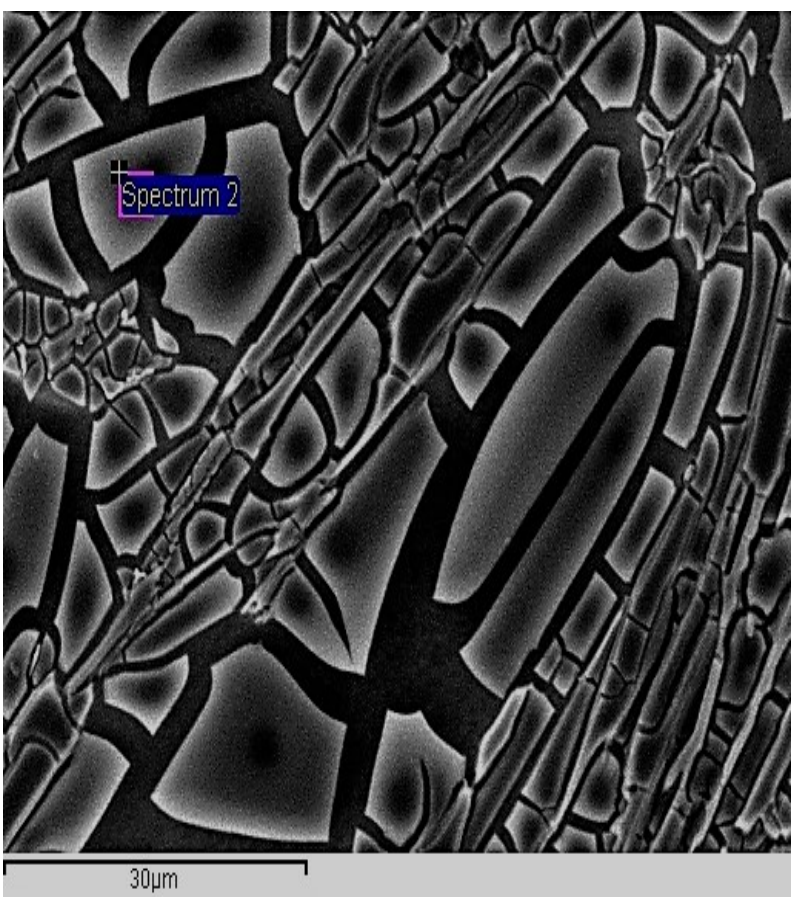

(a)

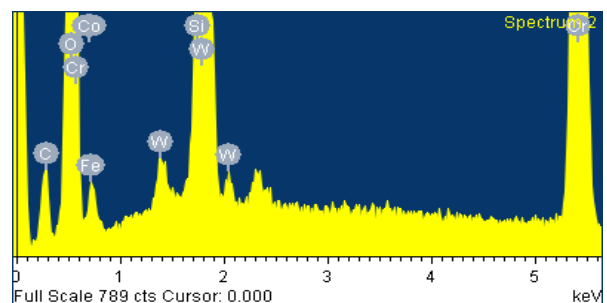

(b)

\begin{tabular}{|c|c|c|}
\hline Element & $\mathbf{w t} \%$ & $\mathbf{a t} \%$ \\
\hline C K & 9.67 & 14.69 \\
\hline O K & 50.38 & 57.46 \\
\hline Si K & 2.02 & 1.31 \\
\hline Cr K & 33.35 & 11.70 \\
\hline Fe K & 13.60 & 4.44 \\
\hline Co K & 21.24 & 6.57 \\
\hline W M & 38.50 & 3.82 \\
\hline Totals & 168.75 & 100.00 \\
\hline
\end{tabular}

(c)

Figure 5-21 EDX results of the white area on the corroded surface of the Stellite 6 alloy sample tested in sodium hydroxide solution: (a) SEM morphology; (b) EDX spectrum; (c) elemental content table

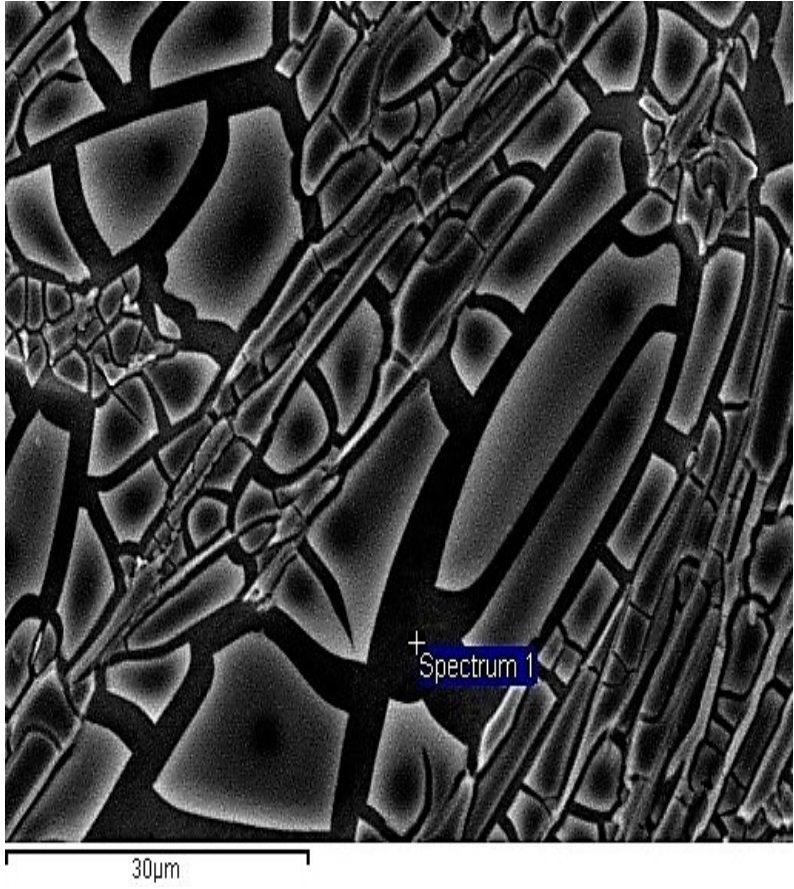

(a)

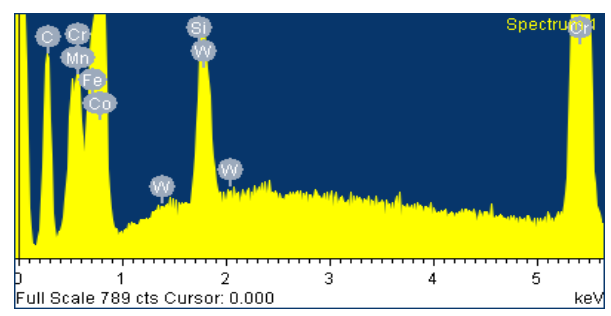

(b)

\begin{tabular}{|c|c|c|}
\hline Element & wt\% & at\% \\
\hline C K & 29.07 & 48.19 \\
\hline Si K & 1.67 & 1.18 \\
\hline Cr K & 39.22 & 15.02 \\
\hline Mn K & 1.29 & 0.47 \\
\hline Fe K & 2.66 & 0.95 \\
\hline Co K & 98.89 & 33.42 \\
\hline W M & 7.08 & 0.77 \\
\hline Totals & 179.87 & 100.00 \\
\hline
\end{tabular}

(c)

Figure 5-22 EDX results of the black area on the corroded surface of the Stellite 6 alloy sample tested in sodium hydroxide solution: (a) SEM morphology; (b) EDX spectrum; (c) elemental content table 


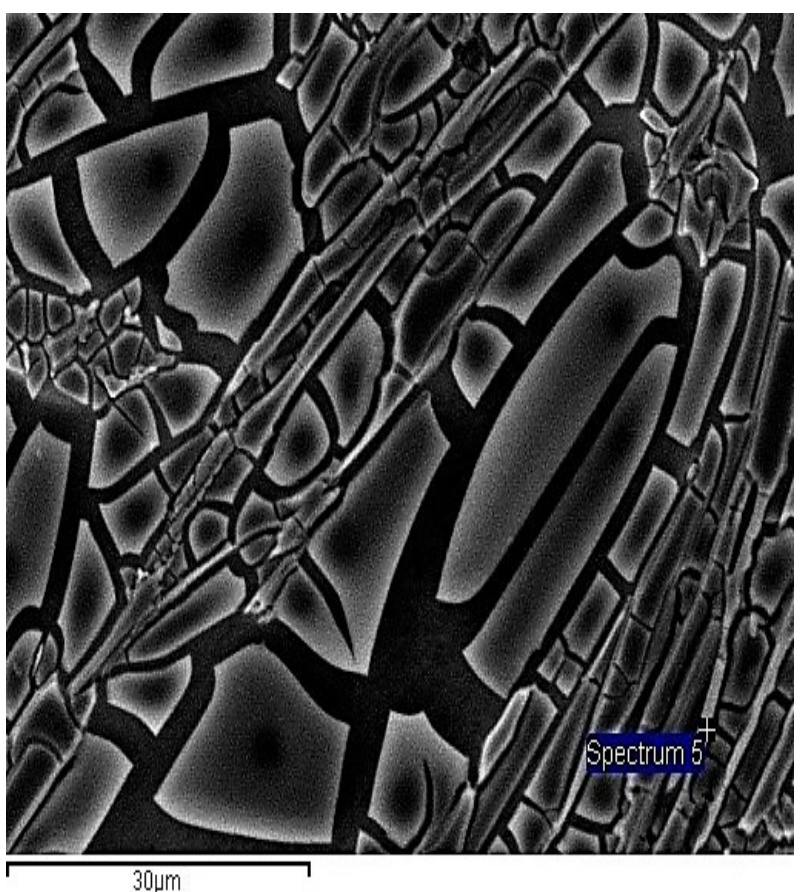

(a)

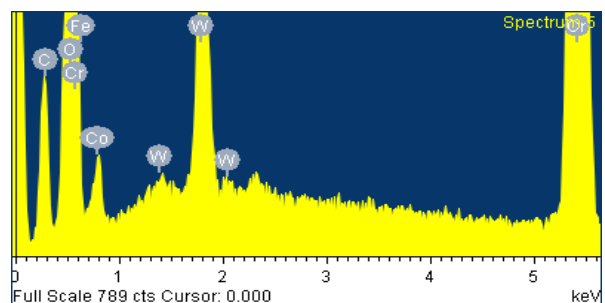

(b)

\begin{tabular}{|c|c|c|}
\hline Element & wt\% & at\% \\
\hline C K & 19.49 & 27.15 \\
\hline O K & 33.75 & 35.30 \\
\hline Cr K & 94.75 & 30.49 \\
\hline Fe K & 2.00 & 0.60 \\
\hline Co K & 17.94 & 5.09 \\
\hline W M & 15.03 & 1.37 \\
\hline Totals & 182.95 & 100.00 \\
\hline
\end{tabular}

(c)

Figure 5-23 EDX results of the grey area on the corroded surface of the Stellite 6 alloy sample tested in sodium hydroxide solution: (a) SEM morphology; (b) EDX spectrum; (c) elemental content table

As seen above, the morphologies of the corroded surfaces of Stellite 6 alloy in the three corrosive media are very similar, and also the chemical compositions of the three areas (white, black and grey) on the surfaces are very close in the three corrosive media conditions, so that the corrosion mechanisms of Stellite 6 alloy in these solutions should be the same, although the corrosion resistance of this alloy to these solutions is not the same, as demonstrated by the current curves of potentiostatic polarization. Based on the EDX results, for the corroded surface of Stellite 6 alloy tested in sodium hydroxide solution, the white area is also mainly $\mathrm{Cr}$-rich oxide, the black area is the substrate of Stellite 6 alloy, and the grey area is Cr-rich oxides and the corrosion product of Cr-rich carbides. 


\subsection{Failure tests of 17-4PH stainless steel}

\subsubsection{Experimental setup}

It was found in the trial test of potentiostatic polarization that $17-4 \mathrm{PH}$ stainless steel could fail in morpholine solution when $4 \mathrm{~V}$ potential to the system was applied after $1500 \mathrm{~s}$, which was indicated by the corrosion product found on the sample surface. The setup for the failure test of $17-4 \mathrm{PH}$ stainless steel in morpholine solution, cyclohexylamine solution, and sodium hydroxide, respectively, was then decided as detailed in Figure 5-24.

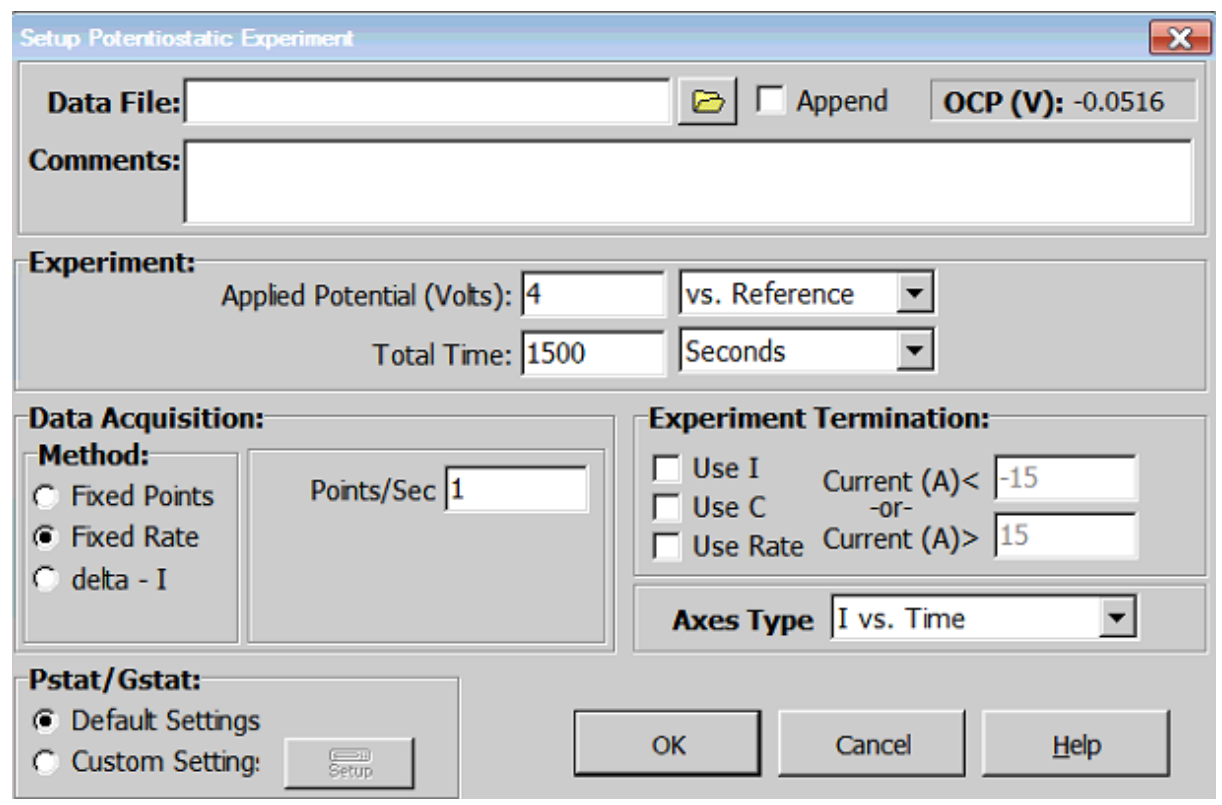

Figure 5-24 Potentiostatic polarization failure test setup for 17-4PH stainless steel

\subsubsection{Failure tests in morpholine solution}

\subsubsection{Polarization test results}

The test duration for 17-4PH stainless steel was much less than that for Stellite 6 alloy, which was determined based on the trial test results. Therefore, the pictures were only taken 
at the start and end of the test, as shown in Figure 5-25. The corresponding polarization curve of $I$ vs. time for the test in morpholine solution is reported in Figure 5-26.

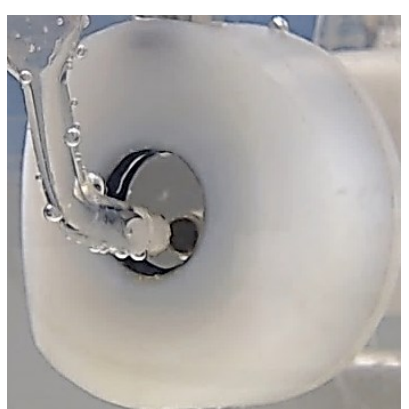

(a)

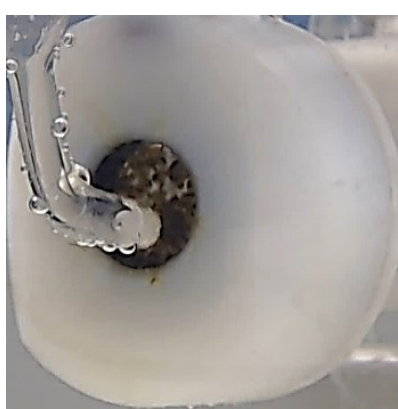

(b)

Figure 5-25 Photos of the 17-4PH stainless steel sample tested in morpholine solution under potentiostatic polarization: (a) for $0 \mathrm{~s}$; (b) for $1500 \mathrm{~s}$

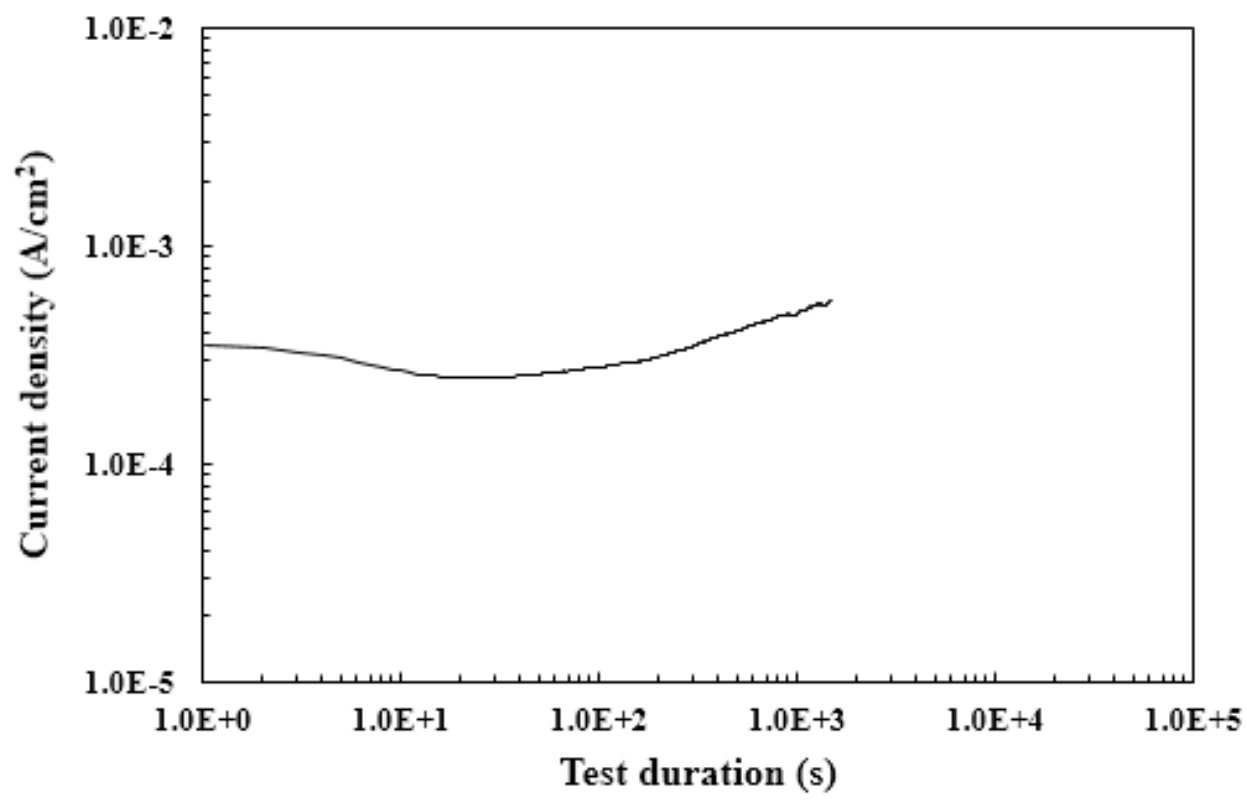

Figure 5-26 Variation of current with time of the 17-4PH stainless steel sample tested in morpholine solution under potentiostatic polarization

It can be seen that the sample surface changed a lot from the start to the end of the test, which indicated the occurrence of corrosion on 17-4PH stainless steel in morpholine solution. According to the current curve in Figure 5-26, an oxide film can form on this 
material once it was in the morpholine solution environment, which was characterized by the decrease in the current, reaching a minimum at the time about $20 \mathrm{~s}$. However, with the corrosion proceeding, the current gradually increased, which implied the damage of the oxide film. On the other hand, comparing the current curves between Stellite 6 alloy (Figure 5-7) and 17-4PH stainless steel (Figure 5-26) in the same corrosive medium - morpholine solution, it is evident that the latter has better oxide film because the current increase of the latter is smaller than that of the former and also the current of the latter exhibited steady growth, but that of the former had a sudden growth with the constant potential applied. This implied that the oxide film on the Stellite 6 surface might be etched suddenly in a short time after it formed but that on the $17-4 \mathrm{PH}$ stainless steel surface was damaged slowly.

\subsubsection{Analyses of corroded surfaces}

SEM/EDX analyses were also performed on the corroded surface of the 17-4PH stainless steel sample tested in morpholine solution. The results are presented in Figure 5-27. Compared with the corroded surface of Stellite 6 alloy in morpholine solution, the damage of oxide film on the $17-4 \mathrm{PH}$ stainless steel surface was less severe, because no large amounts of cracks were observed on the surface of this material. The morphologies of these two corroded surfaces are also very different. The Stellite 6 alloy surface is covered by cracked oxides, but the 17-4PH stainless steel surface contains some porous/loose region and some smoother region as well. EDX analysis was performed on two areas in each region, as shown in Figure 5-28 to Figure 5-31. For the porous region, from the EDX results, the selected two areas (white and grey) all contain very high $\mathrm{O}, \mathrm{Cr}$, and $\mathrm{Fe}$ contents so that 
the porous substances should be the Cr-rich and Fe-rich oxides. However, in the smooth region, although the dark grey area also contains high $\mathrm{O}$ content, the amount is much less in the porous region. Instead, Fe content is very high in this area. Therefore, it can be suggested that this area has some residual oxides but mainly the base material with the oxide film removed. Different from the dark area, the light grey area in the smooth region contains almost no $\mathrm{O}$ but contains very high $\mathrm{Fe}$ and $\mathrm{Cr}$ contents. Thus this area may not have oxides, and it should be purely the base material due to spallation of the oxide film or the oxides were formed in the solution directly, which is away from the sample surface.

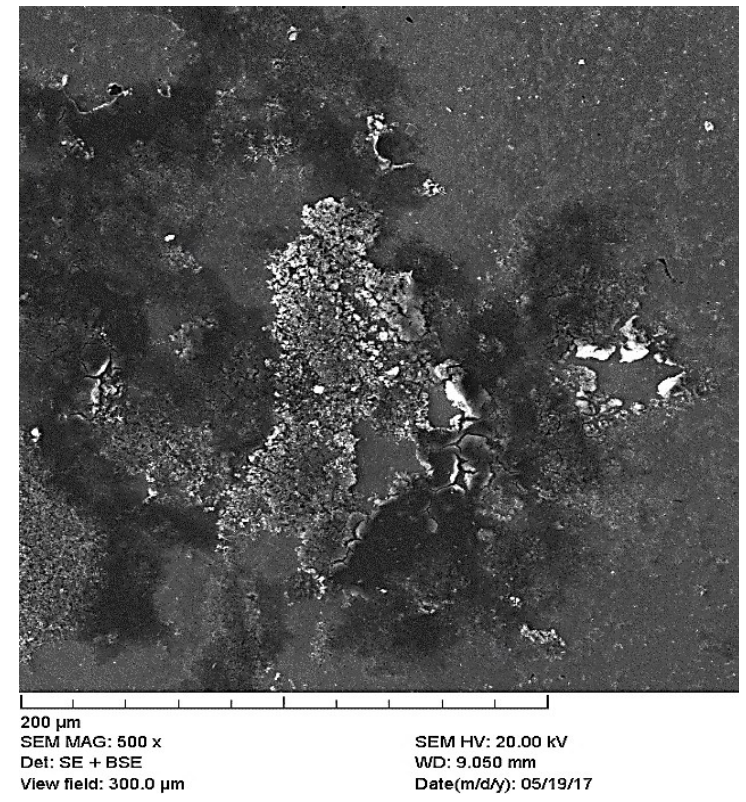

(a)

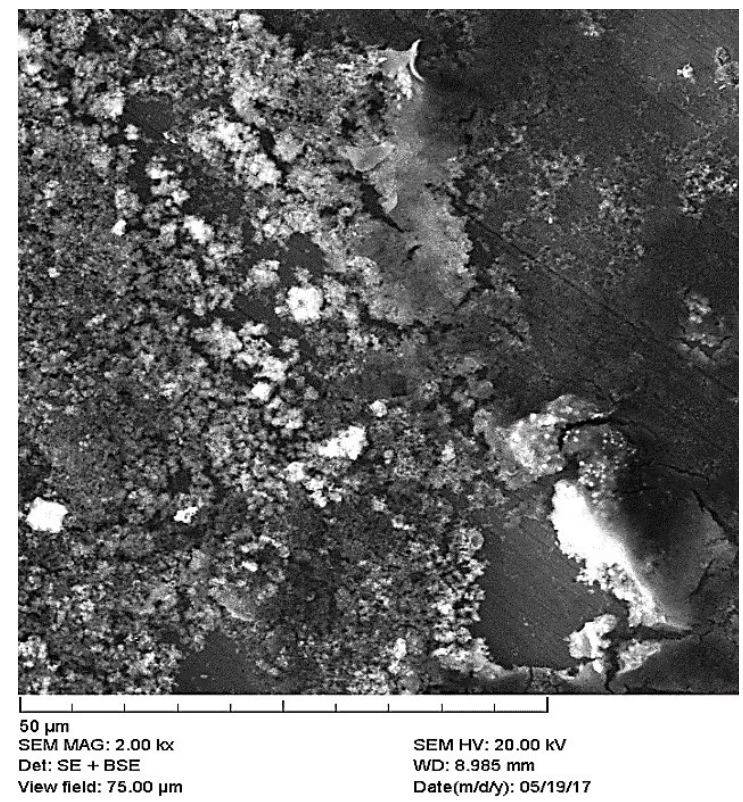

(b)

Figure 5-27 SEM images of corroded surface of the 17-4PH stainless steel sample tested in morpholine solution: (a) at low magnification; (b) at high magnification 


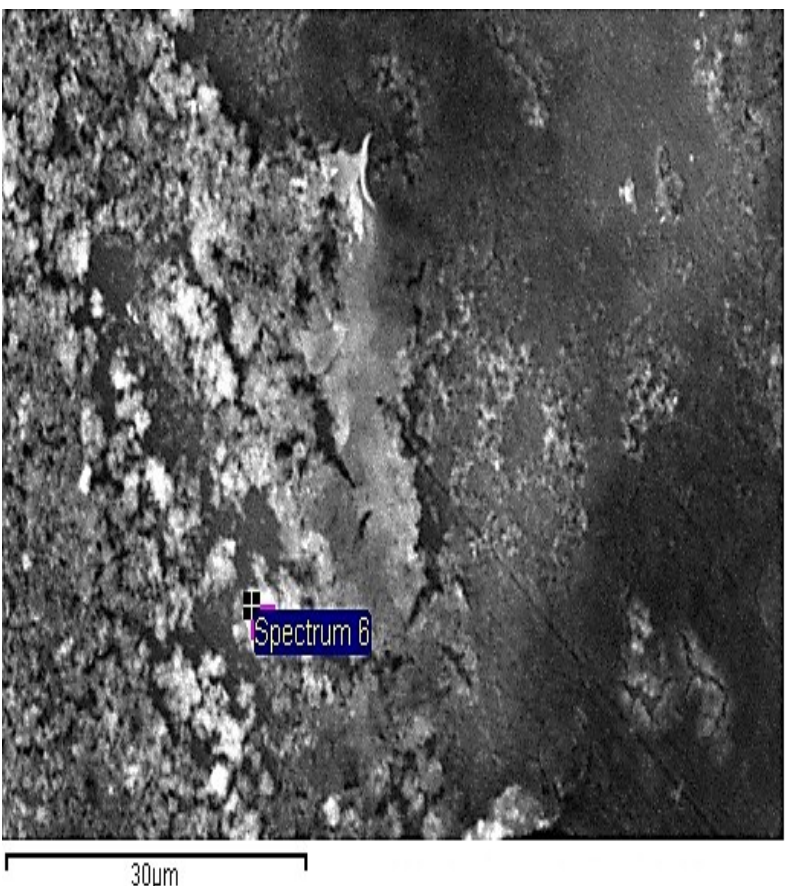

(a)

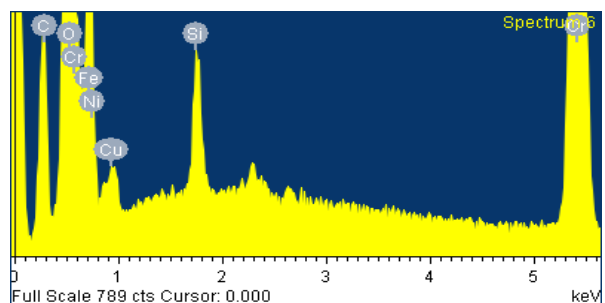

(b)

\begin{tabular}{|c|c|c|}
\hline Element & $\mathbf{w t} \%$ & $\mathbf{a t} \%$ \\
\hline C K & 26.54 & 27.87 \\
\hline O K & 56.19 & 44.29 \\
\hline Si K & 2.54 & 1.14 \\
\hline Cr K & 39.38 & 9.55 \\
\hline Fe K & 69.36 & 15.66 \\
\hline Ni K & 3.64 & 0.78 \\
\hline Cu K & 3.52 & 0.70 \\
\hline Totals & 201.18 & 100.00 \\
\hline
\end{tabular}

(c)

Figure 5-28 EDX results of the white area in the porous region on the corroded surface of the 17-4PH stainless steel sample tested in morpholine solution: (a) SEM morphology;

(b) EDX spectrum; (c) elemental content table

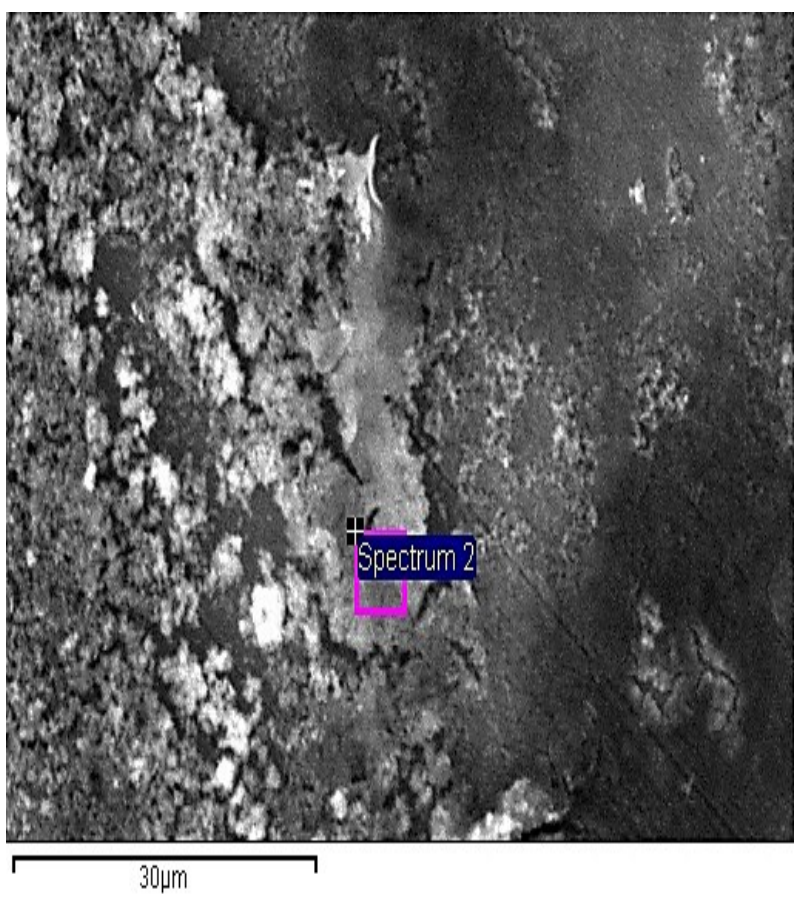

(a)

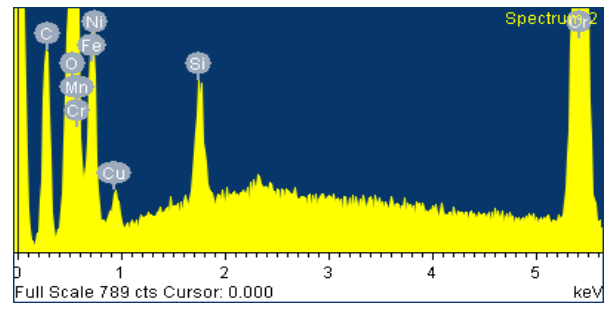

(b)

\begin{tabular}{|c|c|c|}
\hline Element & wt\% & at\% \\
\hline C K & 27.51 & 31.64 \\
\hline O K & 42.91 & 37.04 \\
\hline Si K & 2.17 & 1.07 \\
\hline Cr K & 25.17 & 6.69 \\
\hline Mn K & 1.06 & 0.27 \\
\hline Fe K & 86.33 & 21.35 \\
\hline Ni K & 4.10 & 0.96 \\
\hline Cu K & 4.55 & 0.99 \\
\hline Totals & 193.81 & 100.00 \\
\hline \multicolumn{2}{|c}{}
\end{tabular}

(c)

Figure 5-29 EDX results of the grey area in the porous region on the corroded surface of the 17-4PH stainless steel sample tested in morpholine solution: (a) SEM morphology; (b) EDX spectrum; (c) elemental content table 


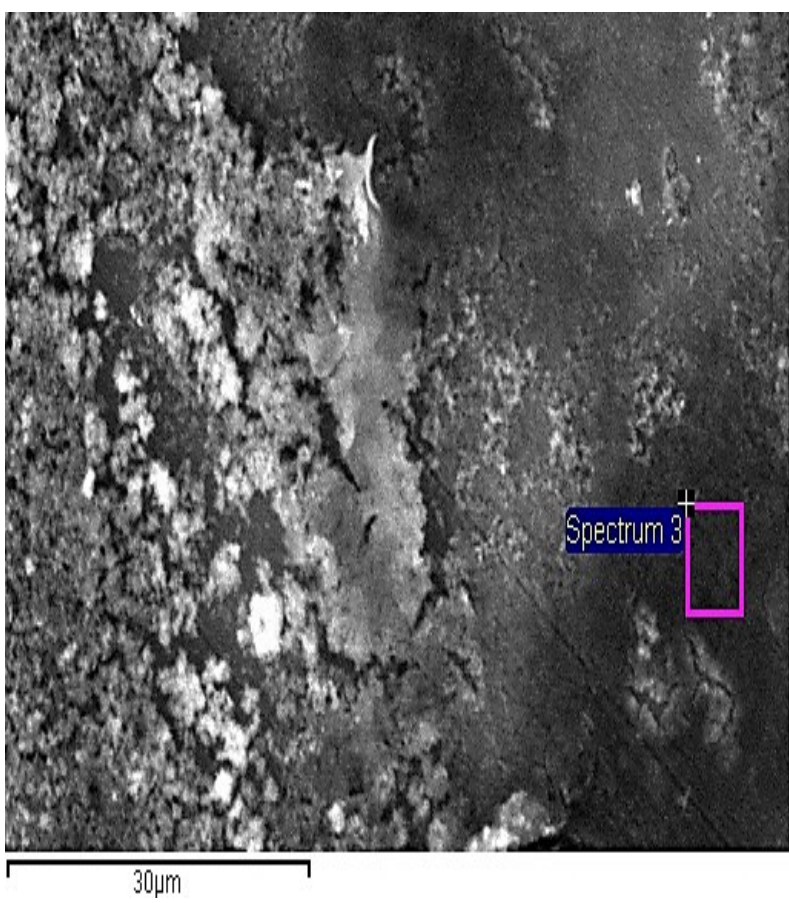

(a)

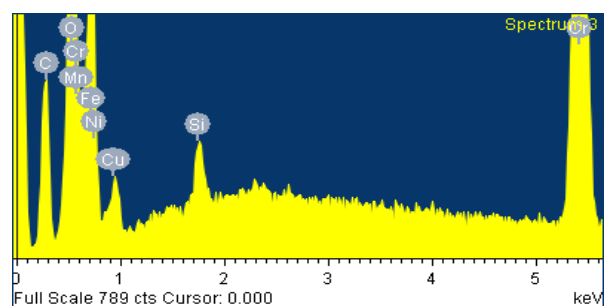

(b)

\begin{tabular}{|c|c|c|}
\hline Element & wt\% & at\% \\
\hline C K & 25.21 & 34.40 \\
\hline O K & 21.31 & 21.84 \\
\hline Si K & 1.15 & 0.67 \\
\hline Cr K & 24.35 & 7.68 \\
\hline Mn K & 1.27 & 0.38 \\
\hline Fe K & 109.95 & 32.27 \\
\hline Ni K & 4.91 & 1.37 \\
\hline Cu K & 5.38 & 1.39 \\
\hline Totals & 193.54 & 100.00 \\
\hline
\end{tabular}

(c)

Figure 5-30 EDX results of the dark grey area in the smooth region on the corroded surface of the 17-4PH stainless steel sample tested in morpholine solution: (a) SEM morphology; (b) EDX spectrum; (c) elemental content table

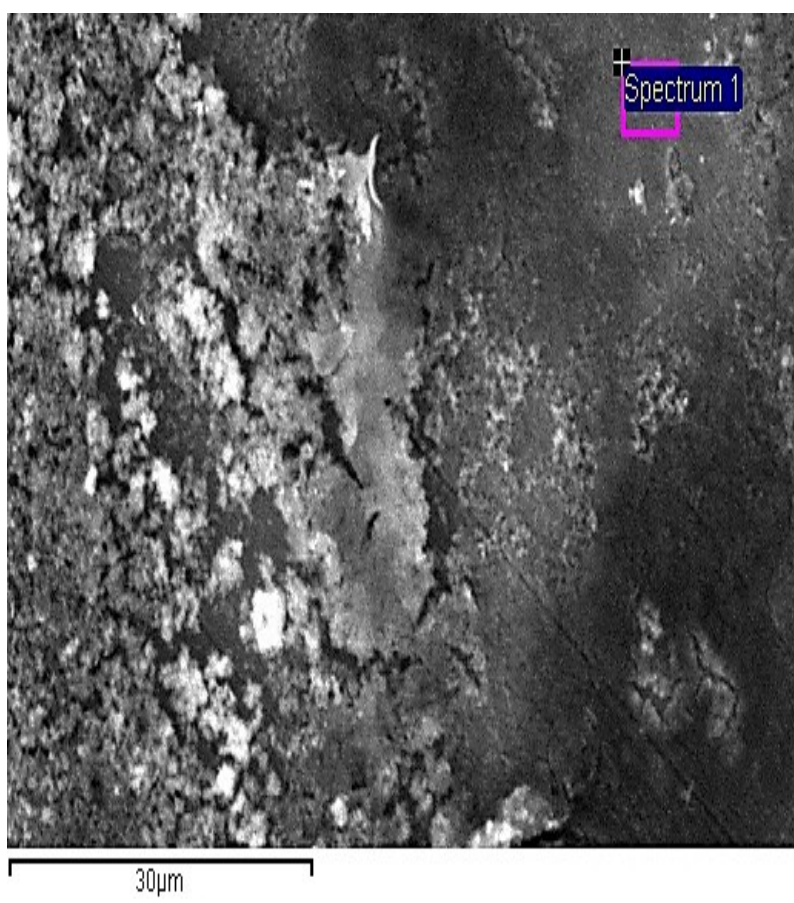

(a)

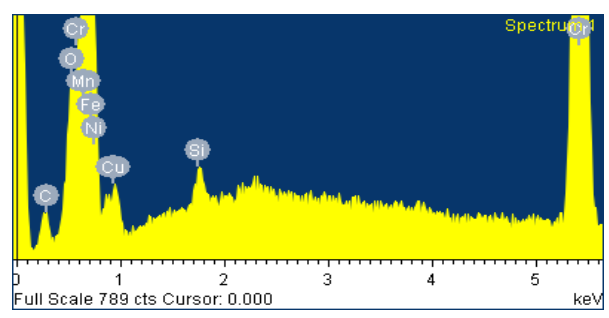

(b)

\begin{tabular}{|c|c|c|}
\hline Element & $\mathbf{w t} \%$ & $\mathbf{a t} \%$ \\
\hline C K & 5.48 & 13.67 \\
\hline O K & 3.00 & 5.62 \\
\hline Si K & 0.73 & 0.78 \\
\hline Cr K & 24.35 & 14.04 \\
\hline Mn K & 1.36 & 0.74 \\
\hline Fe K & 110.70 & 59.42 \\
\hline Ni K & 6.18 & 3.16 \\
\hline Cu K & 5.45 & 2.57 \\
\hline Totals & 157.25 & 100.00 \\
\hline
\end{tabular}

(c)

Figure 5-31 EDX results of the light grey area in the smooth region on the corroded surface of the 17-4PH stainless steel sample tested in morpholine solution: (a) SEM morphology; (b) EDX spectrum; (c) elemental content table 


\subsubsection{Failure tests in cyclohexylamine solution}

\subsubsection{Polarization test results}

Similar to the tests for 17-4PH stainless steel in morpholine solution, the pictures were only taken at the start and end of the test, as shown in Figure 5-32. The corresponding polarization curve is presented in Figure 5-33.

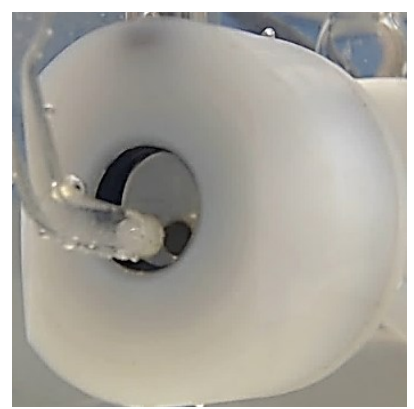

(a)

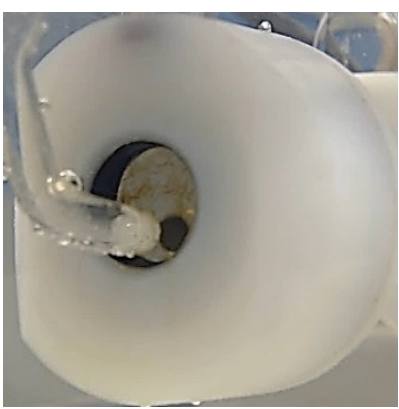

(b)

Figure 5-32 Photos of the 17-4PH stainless steel sample tested in cyclohexylamine solution under potentiostatic polarization: (a) for $0 \mathrm{~s}$; (b) for $1500 \mathrm{~s}$

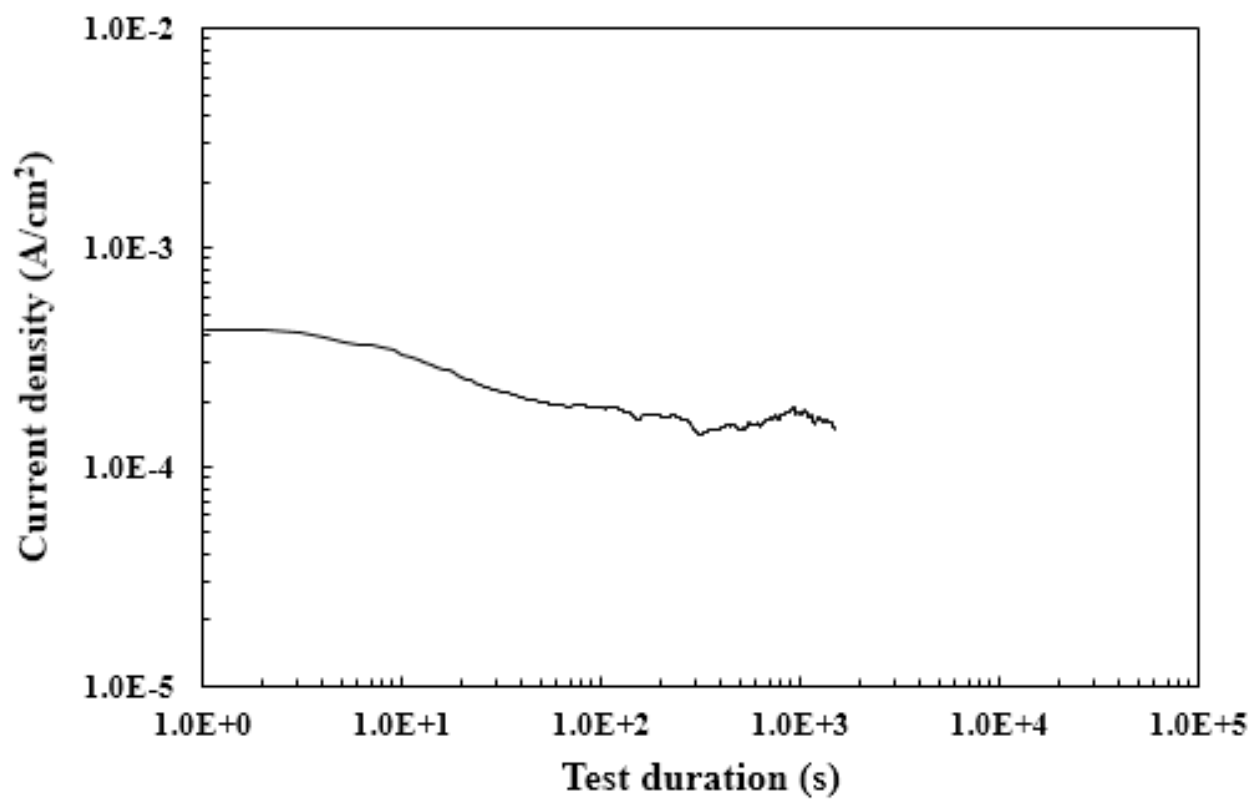

Figure 5-33 Variation of current with time of the 17-4PH stainless steel sample tested in cyclohexylamine solution under potentiostatic polarization 
The corrosion behavior of 17-4PH stainless steel in cyclohexylamine solution differed from that in morpholine solution. It seemed more resistant to corrosion in cyclohexylamine solution than in morpholine solution, because little corrosion product was observed on the sample surface after the test in cyclohexylamine solution, as shown in Figure 5-32. According to the current curve in Figure 5-33, this material can form an oxide film on its surface once it was in the cyclohexylamine solution environment, and this film became more stable with the corrosion proceeding, because the current did not rise up obviously and it was almost unchanged, although fluctuation was observed on the curve. The behavior of this current curve may be due to two reasons. First, the formed oxide film on the sample surface was electron-transferring resistant and could not be damaged with the potential constantly applied. Second, the oxide film was damaged, but it could be repaired rapidly, leading to less corrosion of the base material. The repaired behavior of the oxide film may be related to the slight fluctuation of the current.

\subsubsection{Analyses of corroded surfaces}

Consistent with the polarization curves, the corroded surface of the 17-4PH stainless steel sample tested in cyclohexylamine solution is generally smooth and has no obvious cracks, as shown in Figure 5-34. EDX analysis was performed on the dark and light areas and the results are shown in Figure 5-35 and Figure 5-36. It was found that the dark area contained high $\mathrm{Fe}$ and $\mathrm{Cr}$ contents but almost no $\mathrm{O}$ while the light area contained high $\mathrm{O}, \mathrm{Fe}$, and $\mathrm{Cr}$ contents. These results indicate that the oxide film was not uniform on the 17-4PH stainless steel surface and it may be damaged due to the applied potential but can be repaired quickly. 


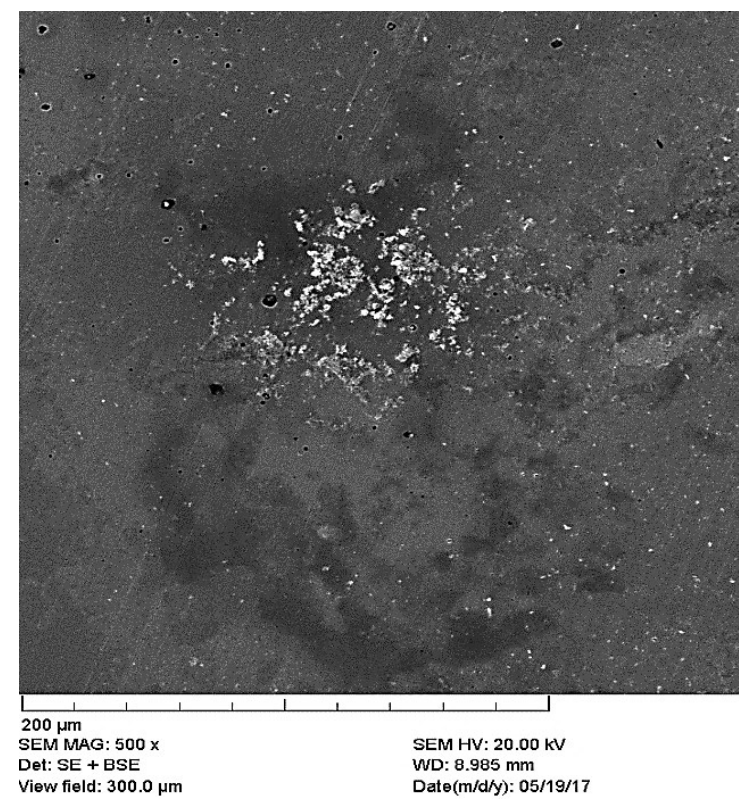

(a)

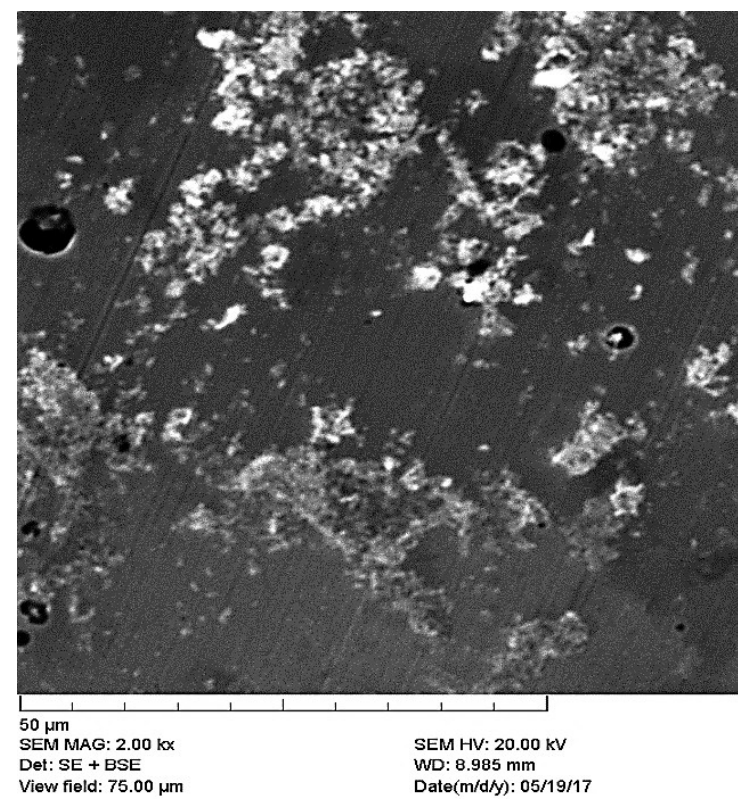

(b)

Figure 5-34 SEM images of corroded surface of the 17-4PH stainless steel sample tested in cyclohexylamine solution: (a) at low magnification; (b) at high magnification

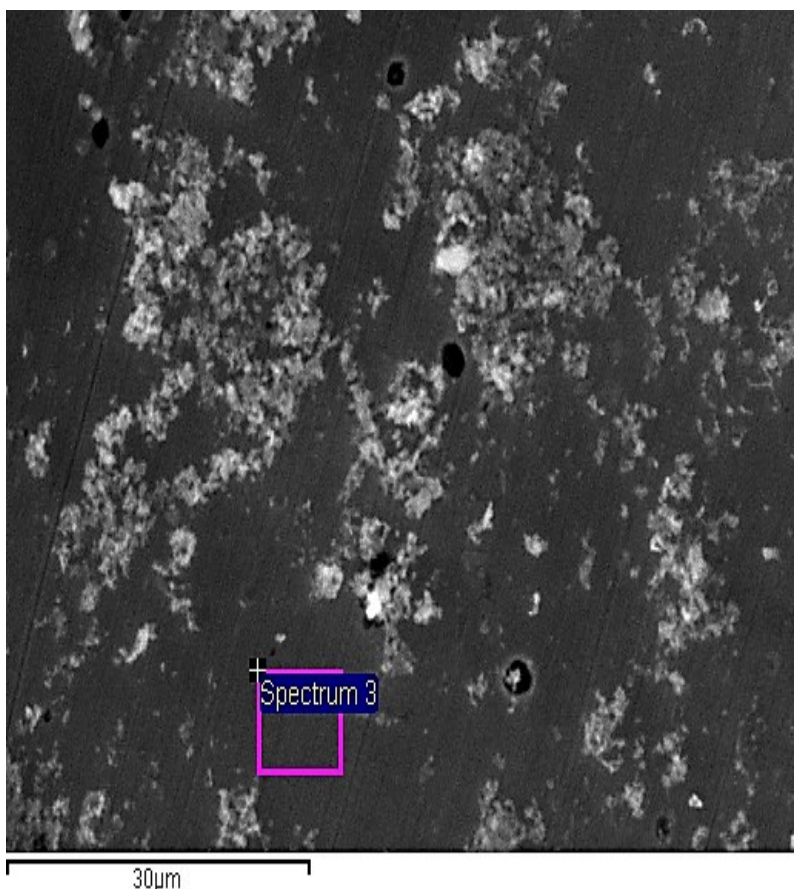

(a)

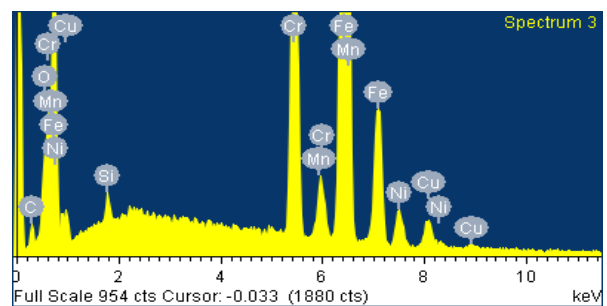

(b)

\begin{tabular}{|c|c|c|}
\hline Element & wt\% & at\% \\
\hline C K & 5.63 & 14.05 \\
\hline O K & 2.95 & 5.54 \\
\hline Si K & 0.88 & 0.94 \\
\hline Cr K & 23.50 & 13.56 \\
\hline Mn K & 1.30 & 0.71 \\
\hline Fe K & 111.22 & 59.73 \\
\hline Ni K & 5.76 & 2.94 \\
\hline Cu K & 5.37 & 2.54 \\
\hline Totals & 156.61 & 100.00 \\
\hline
\end{tabular}

(c)

Figure 5-35 EDX results of the dark area on the corroded surface of the 17-4PH stainless steel sample tested in cyclohexylamine solution: (a) SEM morphology;

(b) EDX spectrum; (c) elemental content table 


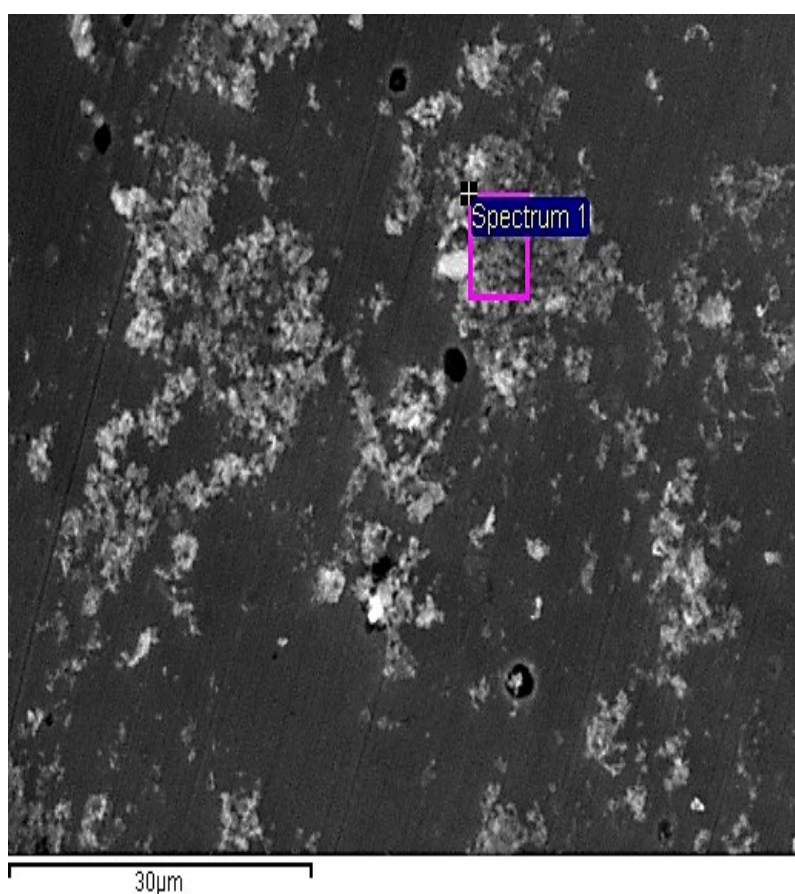

(a)

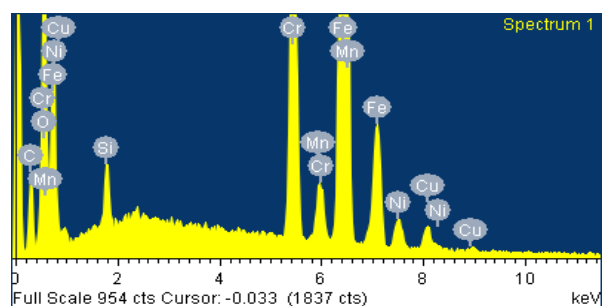

(b)

\begin{tabular}{|c|c|c|}
\hline Element & wt\% & at\% \\
\hline C K & 18.24 & 27.97 \\
\hline O K & 22.00 & 25.33 \\
\hline Si K & 1.98 & 1.30 \\
\hline Cr K & 23.58 & 8.35 \\
\hline Mn K & 1.23 & 0.41 \\
\hline Fe K & 102.33 & 33.75 \\
\hline Ni K & 4.85 & 1.52 \\
\hline Cu K & 4.75 & 1.38 \\
\hline Totals & 178.95 & 100.00 \\
\hline
\end{tabular}

(c)

Figure 5-36 EDX results of the light area on the corroded surface of the 17-4PH stainless steel sample tested in cyclohexylamine solution: (a) SEM morphology;

(b) EDX spectrum; (c) elemental content table

\subsubsection{Failure tests in sodium hydroxide solution}

\subsubsection{Polarization test results}

The pictures taken on the 17-4PH stainless steel sample in sodium hydroxide solution under potentiostatic polarization at the start and end of the test are shown in Figure 5-37. The corresponding polarization curve is presented in Figure 5-38. 


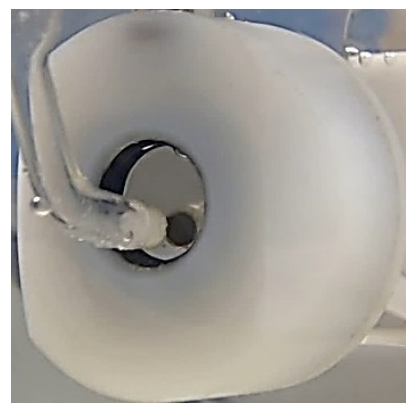

(a)

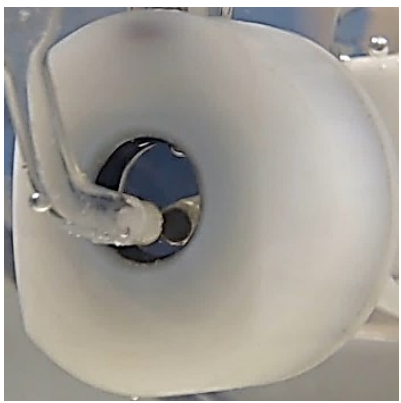

(b)

Figure 5-37 Photos of the 17-4PH stainless steel sample tested in sodium hydroxide solution under potentiostatic polarization: (a) for $0 \mathrm{~s}$; (b) for $1500 \mathrm{~s}$

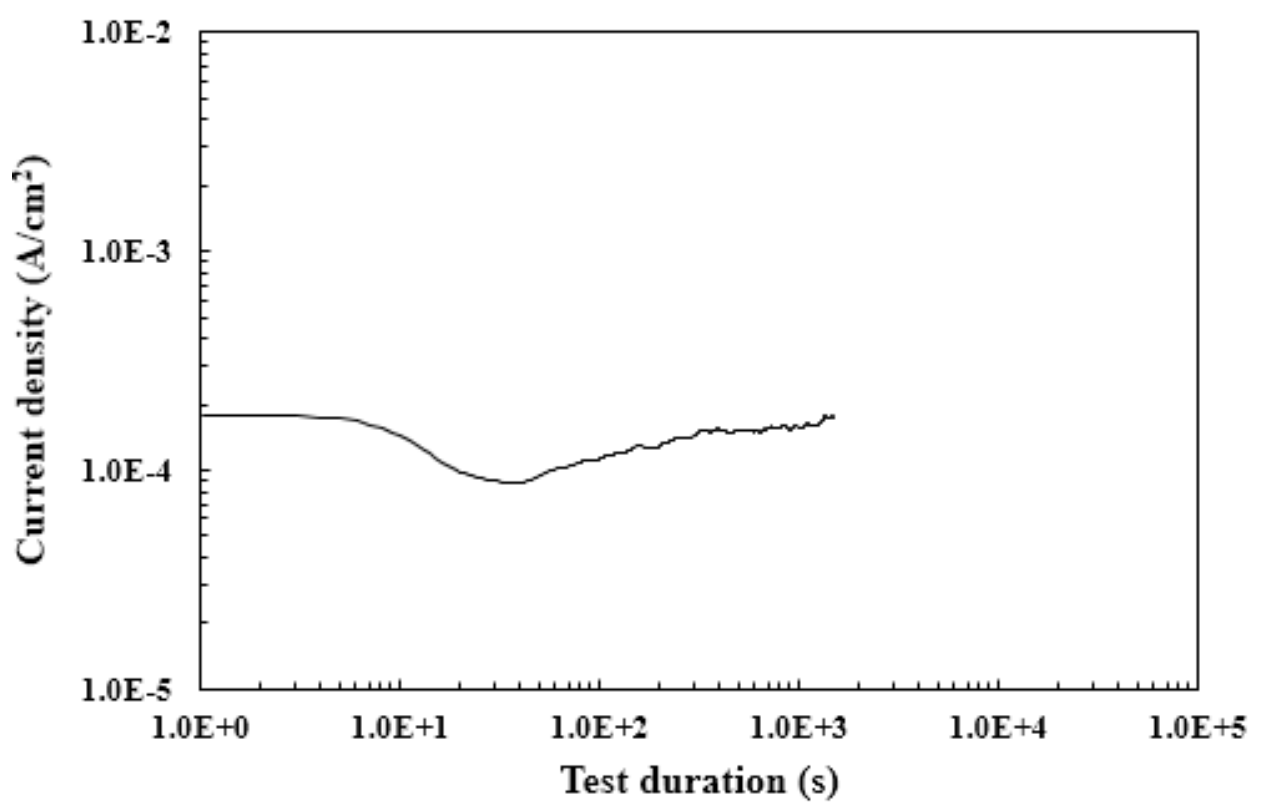

Figure 5-38 Variation of current with time of the 17-4PH stainless steel sample tested in sodium hydroxide solution under potentiostatic polarization

By visual examination, the sample surface was almost not changed, see Figure 5-37. From the current curve, there was a decrease of the current, starting at the time about $8 \mathrm{~s}$ and ending at $40 \mathrm{~s}$ to a minimum, and the current then slightly increased to the original level. This also meant a good oxide film formed on the 17-4PH stainless steel surface in sodium hydroxide solution. 


\subsubsection{Analyses of corroded surfaces}

The SEM images of the corroded surface of 17-4PH stainless steel in sodium hydroxide solution under potentiostatic polarization are shown in Figure 5-39. This surface looks much smoother than those in the morpholine and cyclohexylamine solution tests. Little oxides are found on this surface. EDX analysis was performed on the dark and light areas of the surface, and the results are reported in Figure 5-40 and Figure 5-41. Since no O was detected in the dark area, but very high $\mathrm{Fe}$ and $\mathrm{Cr}$ contents were found, this area should be the base material. On the contrary, high $\mathrm{O}, \mathrm{Fe}$, and $\mathrm{Cr}$ contents were identified in the light area, so that it should be oxides. However, the amount of oxides on this surface is very small.

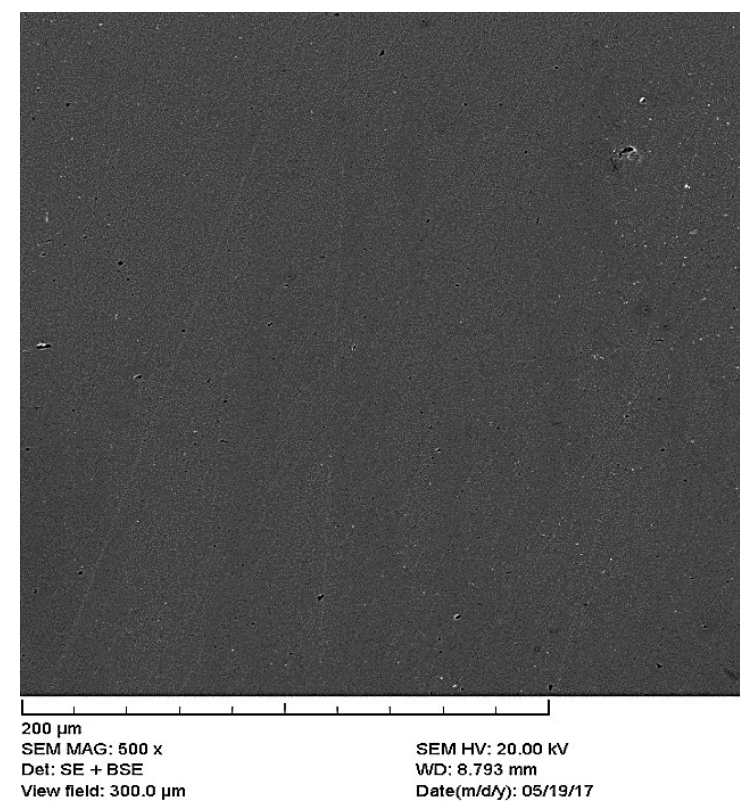

(a)

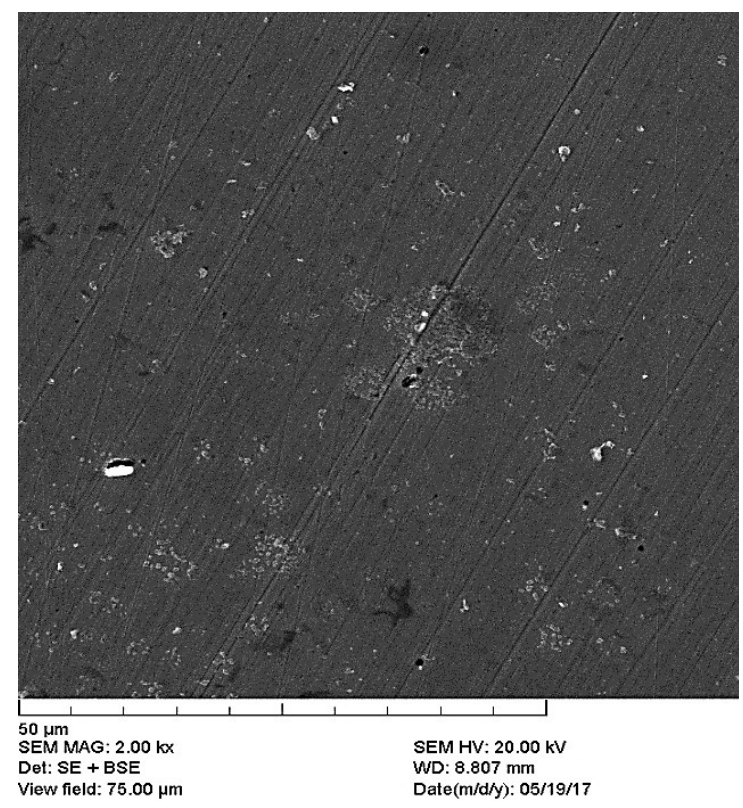

(b)

Figure 5-39 SEM images of corroded surface of the 17-4PH stainless steel sample tested in sodium hydroxide solution: (a) at low magnification; (b) at high magnification 


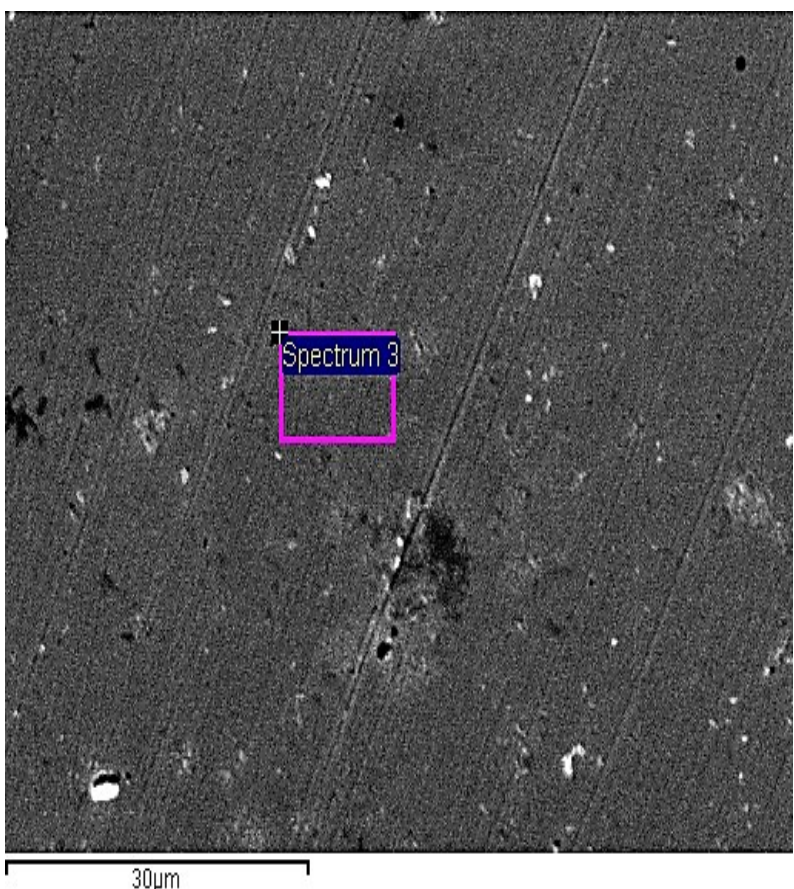

(a)

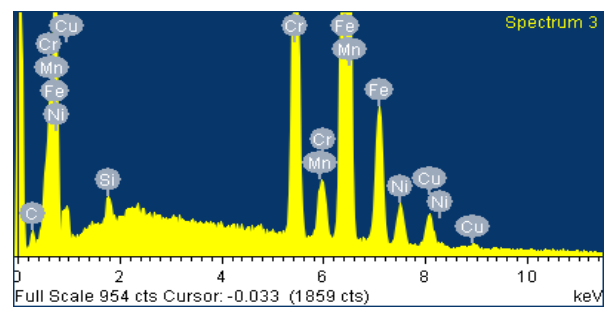

(b)

\begin{tabular}{|c|c|c|}
\hline Element & $\mathbf{w t} \%$ & $\mathbf{a t} \%$ \\
\hline C K & 4.22 & 11.56 \\
\hline Si K & 0.79 & 0.93 \\
\hline Cr K & 24.00 & 15.19 \\
\hline Mn K & 1.39 & 0.83 \\
\hline Fe K & 109.33 & 64.44 \\
\hline Ni K & 6.51 & 3.65 \\
\hline Cu K & 6.58 & 3.41 \\
\hline Totals & 152.81 & 100.00 \\
\hline
\end{tabular}

(c)

Figure 5-40 EDX results of the dark area on the corroded surface of the 17-4PH stainless steel sample tested in morpholine solution: (a) SEM morphology; (b) EDX spectrum;

(c) elemental content table

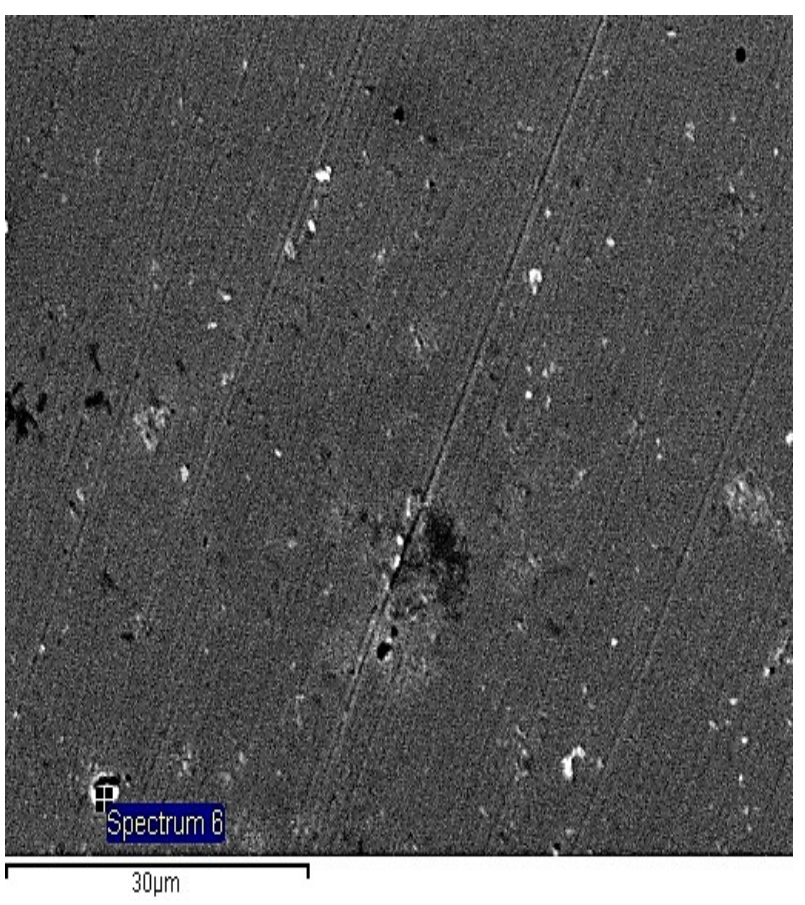

(a)

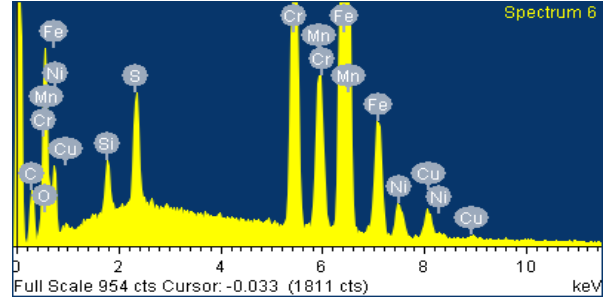

(b)

\begin{tabular}{|c|c|c|}
\hline Element & $\mathbf{w t} \%$ & at\% \\
\hline C K & 16.52 & 30.27 \\
\hline O K & 8.81 & 12.11 \\
\hline Si K & 2.30 & 1.81 \\
\hline Cr K & 23.07 & 9.76 \\
\hline Mn K & 1.33 & 0.53 \\
\hline Fe K & 105.28 & 41.48 \\
\hline Ni K & 5.70 & 2.14 \\
\hline Cu K & 5.50 & 1.91 \\
\hline Totals & 168.52 & 100.00 \\
\hline
\end{tabular}

(c)

Figure 5-41 EDX results of the light area on the corroded surface of the 17-4PH stainless steel sample tested in morpholine solution: (a) SEM morphology; (b) EDX spectrum; (c) elemental content table 


\section{Chapter 6: Discussion on Results}

\subsection{Primary tests}

\subsubsection{Potentiodynamic polarization}

Primary tests discussed here are distinguished from the failure tests. As known from the previous chapters, in the primary tests the maximum potential applied was $1.2 \mathrm{~V}$, but in the failure tests the applied constant potential was much higher $(4 \mathrm{~V})$. Based on the potentiodynamic polarization curves in Figure 4-1, Figure 4-2, Figure 4-7, Figure 4-8, Figure 4-13 and Figure 4-14, both Stellite 6 alloy and 17-4PH stainless steel have the feature or tendency of corrosion passivation due to forming a protective oxide film on their surfaces. Within the passive region of the polarization curve, with increasing potential, current is unchanged or slightly changed. However, the passive region of 17-4PH stainless steel is more distinct than that of Stellite 6 alloy, because the slopes of phase 2 of the polarization curves for Stellite 6 alloy have lower values than that for 17-4PH stainless steel.

Furthermore, from Table 4-1, the corrosion potential $E_{\text {corr }}$ of Stellite 6 alloy is slightly higher than that of $17-4 \mathrm{PH}$ stainless steel. There is almost no difference in the current density $I_{c o r r}$ between Stellite 6 alloy and 17-4PH stainless steel. The polarization resistance of Stellite 6 alloy is larger than that of 17-4PH stainless steel. It can be suggested that Stellite 6 alloy is more resistant to initial oxidation in morpholine solution than $17-4 \mathrm{PH}$ stainless steel, but once the corrosion starts, Stellite 6 alloy and 17-4PH stainless steel exhibit similar corrosion behavior with similar current density. Generally, Stellite 6 alloy 
has better corrosion resistance in morpholine solution than $17-4 \mathrm{PH}$ stainless steel at a $\mathrm{pH}$ level around 9.5 because it has a larger resistance $\left(R_{p}\right)$ value.

In cyclohexylamine solution, as shown in Table 4-2, Stellite 6 alloy and 17-4PH stainless steel have close values of $E_{\text {corr }}$ and Stellite 6 alloy has a smaller value of $I_{c o r r}$ and a much larger value of polarization resistance $R_{p}$ than $17-4 \mathrm{PH}$ stainless steel. Therefore, these two materials exhibit similar resistance to initial corrosion in cyclohexylamine solution, but once the corrosion occurs, it is more severe on 17-4PH stainless steel.

For sodium hydroxide solution, as reported in Table 4-3, the $E_{\text {corr }}$ of Stellite 6 alloy is lower than that of 17-4PH stainless steel, indicating the easier start of corrosion on Stellite 6 alloy. However, the smaller $I_{\text {corr }}$ value and larger $R_{p}$ value of Stellite 6 alloy mean that it is overall better than 17-4PH stainless steel in corrosion resistance to sodium hydroxide solution at a $\mathrm{pH}$ level around 9.5.

Further comparison for each material can be made between the three corrosive media. The slopes of divided phases of the polarization curves for Stellite 6 alloy and 17-4PH stainless steel are summarized in Table 6-1. For Stellite 6 alloy, it is the most resistant to corrosion in sodium hydroxide solution because the polarization curve has phase 2 and phase 4 with larger slopes, closer to passivation. The slope values of three phases for Stellite 6 alloy in the other solutions are similar, which indicates equivalent corrosion resistance. As for $17-4 \mathrm{PH}$ stainless steel, it behaves similarly in the three solutions because the polarization curves all have four phases, showing obvious passive region and oxide film repair behavior. 
Table 6-1 The slopes of divided phases of polarization curves for Stellite 6 alloy and 17-4PH stainless steel

\begin{tabular}{|c|c|c|c|c|c|}
\hline \multirow{2}{*}{ Specimen } & \multirow{2}{*}{ Corrosive medium } & \multicolumn{4}{|c|}{$\boldsymbol{\beta}(\mathbf{V} / \mathbf{d e c}$ de) } \\
\cline { 3 - 6 } & & $\mathbf{1}$ & $\mathbf{2}$ & $\mathbf{3}$ & $\mathbf{4}$ \\
\hline \multirow{3}{*}{ Stellite 6 } & Morpholine & 0.21 & 0.34 & 0.66 & $/$ \\
\cline { 2 - 6 } & Cyclohexylamine & 0.19 & 0.64 & 0.51 & $/$ \\
\cline { 2 - 6 } & Sodium hydroxide & 0.25 & 1.21 & 0.32 & 0.94 \\
\hline \multirow{3}{*}{ 17-4PH } & Morpholine & 0.27 & 3.59 & 0.22 & 0.63 \\
\cline { 2 - 6 } & Cyclohexylamine & 0.29 & 1.01 & 0.19 & 0.79 \\
\cline { 2 - 6 } & Sodium hydroxide & 0.28 & 1.52 & 0.21 & 0.55 \\
\hline
\end{tabular}

The average values of polarization parameters for Stellite 6 alloy and 17-4PH stainless steel in the three corrosive media are summarized in Table 6-2. For Stellite 6 alloy, according to the $E_{\text {corr }}$ values, it is the most resistant to initial corrosion (oxidation) in morpholine solution and the least in sodium hydroxide solution, but once the corrosion occurs, Stellite 6 alloy will be oxidized the most severe in morpholine solution and the least in cyclohexylamine solution, according to the $I_{\text {corr }}$ and $R_{p}$ values. Regarding $17-4 \mathrm{PH}$ stainless steel, it behaves similarly in the three solutions at the same $\mathrm{pH}$ level around 9.5, according to the close $E_{\text {corr }}, I_{\text {corr }}$ and $R_{p}$ values, as seen in Table 6-2. The numbers in parentheses are standard deviation errors in units of the least significant digit.

Table 6-2 Average polarization parameters for Stellite 6 alloy and 17-4PH stainless steel

\begin{tabular}{|c|c|c|c|c|}
\hline Specimen & Corrosive medium & $\begin{array}{c}\mathbf{E}_{\text {corr }} \\
\text { (V vs. SCE) }\end{array}$ & $\begin{array}{c}\mathbf{I}_{\mathbf{c o r r}} \\
\left(\mathbf{n A} / \mathbf{c m}^{\mathbf{2}}\right)\end{array}$ & $\begin{array}{c}\mathbf{R}_{\mathbf{p}} \\
\left(\mathbf{M} \boldsymbol{\Omega} \cdot \mathbf{c m}^{\mathbf{2}}\right)\end{array}$ \\
\hline \multirow{3}{*}{ Stellite 6 } & Morpholine & $-0.09(2)$ & $20.8(3)$ & $2.7(7)$ \\
\cline { 2 - 5 } & Cyclohexylamine & $-0.16(1)$ & $7.5(5)$ & $4.8(2)$ \\
\cline { 2 - 5 } & Sodium hydroxide & $-0.23(1)$ & $10.1(1)$ & $3.4(2)$ \\
\hline \multirow{3}{*}{ 17-4PH } & Morpholine & $-0.17(1)$ & $22(5)$ & $1.5(2)$ \\
\cline { 2 - 5 } & Cyclohexylamine & $-0.18(2)$ & $25(4)$ & $1.3(2)$ \\
\cline { 2 - 5 } & Sodium hydroxide & $-0.19(1)$ & $26(3)$ & $1.3(2)$ \\
\hline
\end{tabular}




\subsubsection{Cyclic polarization}

As illustrated in Figure 4-5, Figure 4-11 and Figure 4-17, the cyclic polarization curves of Stellite 6 alloy and 17-4PH stainless steel in morpholine, cyclohexylamine and sodium hydroxide solutions all do not have an electropositive hysteresis loop, which means that these materials may suffer pitting corrosion. Since the cyclic polarization curves of $17-4 \mathrm{PH}$ stainless steel in the three media all have a large electronegative hysteresis loop, while those of Stellite 6 not, the latter is better than the former against pitting corrosion. The erosion/corrosion could also result in pits, thus better resistance against pitting corrosion may indicate better resistance to erosion/corrosion as well.

\subsubsection{EIS}

The key parameter that characterizes corrosion behavior of materials using EIS is the charge transfer resistance, $R_{c t}$. The larger the $R_{c t}$ value, the better the corrosion resistance of the material is. Based on this assumption, both Stellite 6 alloy and 17-4PH stainless steel are more sensitive to morpholine solution than to cyclohexylamine and sodium hydroxide solutions, as demonstrated by the values of charge transfer resistance $R_{c t}$ in Table 6-3. Between Stellite 6 alloy and 17-4PH stainless steel in different corrosive media, Stellite 6 alloy is better than 17-4PH stainless steel in morpholine solution and almost doubly better in the other solutions. In general, the EIS results are consistent with the polarization results. The numbers in parentheses are standard deviation errors in units of the least significant digit. 
Table 6-3 Average charge transfer resistances of Stellite 6 alloy and 17-4PH stainless steel

\begin{tabular}{|l|c|c|}
\hline Specimen & Corrosive medium & $\begin{array}{c}\mathbf{R}_{\mathbf{c t}} \\
\left(\mathbf{M} \boldsymbol{\Omega} \cdot \mathbf{c m}^{\mathbf{2}} \mathbf{)}\right.\end{array}$ \\
\hline \multirow{3}{*}{ Stellite 6 } & Morpholine & $1.9(4)$ \\
\cline { 2 - 3 } & Cyclohexylamine & $5.4(6)$ \\
\cline { 2 - 3 } & Sodium hydroxide & $5.6(7)$ \\
\hline \multirow{3}{*}{ 17-4PH } & Morpholine & $1.7(2)$ \\
\cline { 2 - 3 } & Cyclohexylamine & $3.4(9)$ \\
\cline { 2 - 3 } & Sodium hydroxide & $2.9(4)$ \\
\hline
\end{tabular}

\subsection{Failure tests}

\subsubsection{Visual observation of sample surfaces}

Although primary polarization tests had provided general corrosion properties of Stellite 6 alloy and 17-4PH stainless steel in morpholine, cyclohexylamine and sodium hydroxide solutions, since these materials are more resistant to basic media than to acid media [25], no obvious damage was observed on the surfaces of the samples in the primary polarization tests. Moreover, in general, Stellite 6 alloy exhibits better corrosion resistance than 17-4PH stainless steel in the three corrosive media, as seen in Table 6-2 and Table 6-3, but the difference between these two materials is not significant in the primary tests. In order to discover the damage behavior and hence better understand the corrosion mechanisms of Stellite 6 alloy and 17-4PH stainless steel in morpholine, cyclohexylamine and sodium hydroxide solutions at the same $\mathrm{pH}$ level around 9.5, as well as to further investigate the difference in corrosion behavior between these two materials in such corrosive conditions, failure tests were conducted on these materials in the same corrosive media condition. Compared with primary tests, in the failure tests, the applied potential was higher $(4 \mathrm{~V})$ to generate corrosion damage of the sample surface. 
As discussed above, the primary test results show that Stellite 6 alloy exhibits better corrosion resistance than $17-4 \mathrm{PH}$ stainless steel in morpholine, cyclohexylamine and sodium hydroxide solutions. Consistently, in the failure tests, with the higher potential applied, the Stellite 6 alloy surface did not show obvious change by visual examination until $10000 \mathrm{~s}$ after the test, but the 17-4PH stainless steel surface showed severe corrosion product after $1500 \mathrm{~s}$ test in morpholine solution and some corrosion product after $1500 \mathrm{~s}$ test in cyclohexylamine solution. In sodium hydroxide solution, the change of the 17-4PH stainless steel surface was not obvious after $1500 \mathrm{~s}$ test by visual examination.

\subsubsection{Polarization curves and corroded surfaces}

Further analyses of the polarization current curves confirmed the passivation feature of Stellite 6 alloy and 17-4PH stainless steel in morpholine, cyclohexylamine and sodium hydroxide solutions. As demonstrated in Figure 6-1, the currents all have a decrease during the tests in the three solutions, which indicates the formation of oxide films on the sample surfaces. For Stellite 6 alloy, with the higher potential continuously applied, the current would increase from the minimum to a high level, which was due to the break of the protective oxide film on the sample surface. From the SEM analyses of the corroded surfaces, the oxide films on Stellite 6 surfaces in the three media were entirely etched, leading to corrosion of the base material. It is assumed that the more electron-transferring resistant of the oxide film on the sample, the smaller the current value is. Morpholine has the strongest effect among all tested solutions for Stellite 6 alloy. There is no difference observed on the corroded surfaces of Stellite 6 alloy in the three corrosive solutions; they all show large cracked or etched oxides (Figure 5-8, Figure 5-14 and Figure 5-20). 


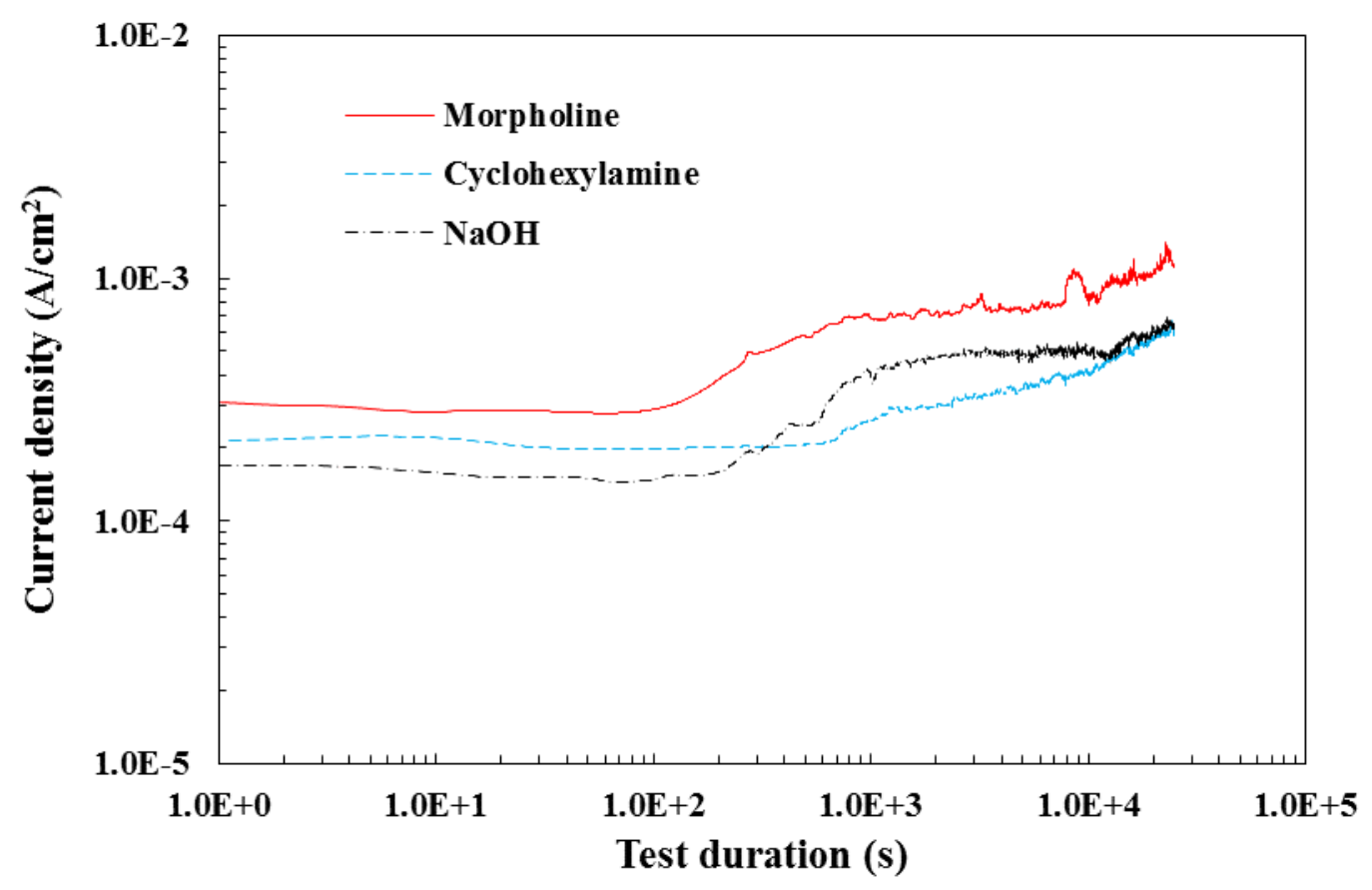

Figure 6-1 Variation of current with time for Stellite 6 alloy under potentiostatic polarization

However, 17-4PH stainless steel behaved somewhat differently from Stellite 6 alloy in the failure tests. First, the current of 17-4PH stainless steel did not increase much from the minimum with the higher potential continuously applied, as shown in Figure 6-2. Second, the corroded surfaces of 17-4PH stainless steel were much smoother than that of Stellite 6 alloy and did not show largely cracked oxides. It seemed that the oxide film on $17-4 \mathrm{PH}$ stainless steel was only partially damaged, as seen in Figure 5-27, Figure 5-34 and Figure 5-39. From the current curves of Stellite 6 alloy and 17-4PH stainless steel, the times when the current had a minimum for the two materials were very close, but the current of 174PH stainless steel increased very little or did not increase with the higher potential continuously applied. Combining the observations of the corroded surfaces, it can be suggested that 17-4PH stainless steel had better corrosion resistance than Stellite 6 alloy 
under a high potential owing to the more electron-transferring resistant oxide film bonded on its surface. This is also consistent with the primary tests, the polarization curves of 17-4PH stainless steel have the evident passive region within the tested potential range, indicating a good oxide film formed on its surface. Since the testing period for 17-4PH stainless steel is shorter than that for Stellite 6 alloy, it is possible for 17-4PH stainless steel to experience a dramatic current increase when the testing period is extended. Morpholine has the strongest effect among all tested solutions for 17-4PH stainless steel.

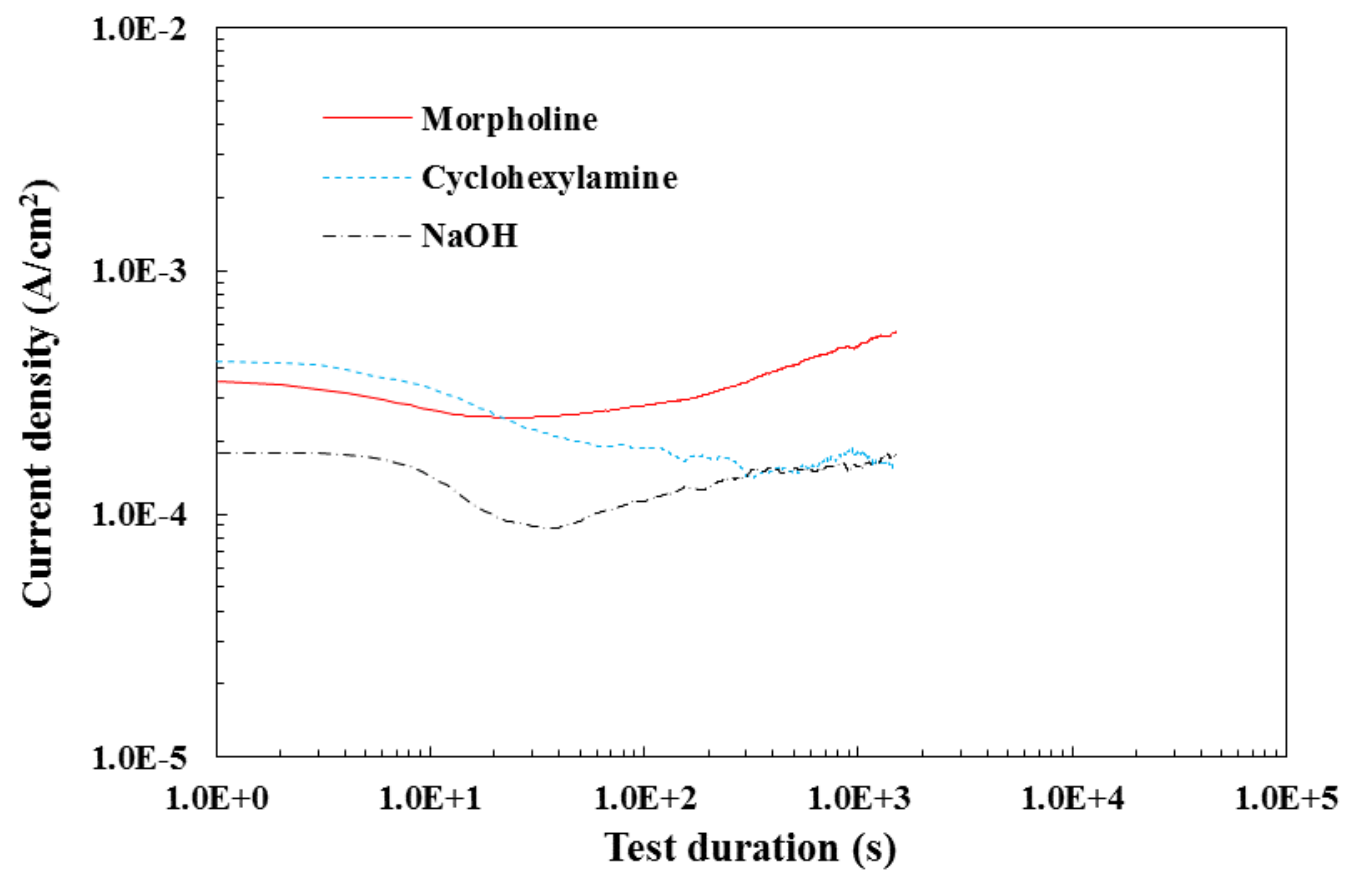

Figure 6-2 Variation of current with time for 17-4PH stainless steel under potentiostatic polarization 


\subsection{Hypotheses of corrosion mechanisms}

\subsubsection{Oxide film formation}

Stellite 6 alloy and 17-4PH stainless steel are both corrosion-resistant because they contain high $\mathrm{Co}$ and $\mathrm{Cr}$ or $\mathrm{Fe}$ and $\mathrm{Cr}$ contents, which enable the formation of adherent oxide films on their surfaces. Morpholine, cyclohexylamine all belong to amine class, and sodium hydroxide is an alkali, which are basic in nature. Due to the presence of oxygen content, Stellite 6 alloy and 17-4PH stainless steel can form Cr-rich and Co-rich or Cr-rich and Fe-rich oxide films. $\mathrm{Cr}_{2} \mathrm{O}_{3}$ is the main oxide in the films as $\mathrm{Cr}$ is the most active element to react with $\mathrm{O}$ among the alloy elements [97]. It is also considered to be dense and electron-transferring resistant, having protective effects against further corrosion of the base material [98]. Therefore, whether the $\mathrm{Cr}_{2} \mathrm{O}_{3}$ film is strongly bonded to the metal surface or not determines the corrosion resistance of the material. From the primary tests under lower potentials applied, Stellite 6 alloy exhibited better corrosion or oxidation resistance than $17-4 \mathrm{PH}$ stainless steel in morpholine, cyclohexylamine and sodium hydroxide solutions, but in the failure tests under a higher potential, 17-4PH stainless steel showed better corrosion resistance with better oxide film behavior. According to the chemical compositions of Stellite 6 alloy and 17-4PH stainless steel, the Cr content in the former is higher, but Stellite 6 alloy contains large amounts of Cr-rich carbides, which reduces the solute $\mathrm{Cr}$ of the solid solution. The $\mathrm{Cr}$ in $\mathrm{Cr}_{2} \mathrm{O}_{3}$ comes from solute $\mathrm{Cr}$ in the solid solution and not from the Cr-rich carbides. Thus the oxide film formed on Stellite 6 alloy may not be uniform and will be etched into pieces when it is damaged. On the contrary, 17-4PH stainless steel has an entire martensitic structure, and solute $\mathrm{Cr}$ is uniformly distributed in the structure, leading to a uniform oxide film on 17-4PH stainless steel. As a 
result, it is removed from the surface due to spallation when it is damaged, or the oxides would form in the solution, which is away from the sample surface.

\subsubsection{Pourbaix diagrams}

To further analyze the corrosion mechanisms of Stellite 6 alloy and 17-4PH stainless steel in the failure tests, Pourbaix diagrams showing the potential-pH equilibrium of $\mathrm{Co}, \mathrm{Cr}$ and Fe are utilized, as shown in Figure 6-3 [99, 100], Figure 6-4 [101], and Figure 6-5 [102, 103]. In this research, the morpholine, cyclohexylamine and sodium hydroxide solutions were adjusted to be $\mathrm{pH} 9.5$ and the potential was set to be $4 \mathrm{~V}$ in the failure tests. Thus, according to Pourbaix diagrams, at the potential of $4 \mathrm{~V}_{\mathrm{SCE}}$ and the $\mathrm{pH}$ of 9.5 of the corrosive solutions, the Stellite 6 alloy sample released chromite ions $\mathrm{CrO}_{4}{ }^{2-}$ and the Co element formed cobalt hydroxide $\mathrm{Co}(\mathrm{OH})_{3}$ when reacting with the corrosive solutions. Therefore, the residuals (corrosion product) left on the corroded surfaces of Stellite 6 alloy in the failure tests should be the mixture of major $\mathrm{Cr}_{2} \mathrm{O}_{3}$, minor $\mathrm{Co}_{3} \mathrm{O}_{4}$ and cobalt hydroxide $\mathrm{Co}(\mathrm{OH})_{3}$. The chromite ions $\mathrm{CrO}_{4}{ }^{2-}$ dissolved into the solutions could change the color of the solutions to yellow. For 17-4PH stainless steel, at the potential of $4 \mathrm{~V}_{\mathrm{SCE}}$ and the $\mathrm{pH}$ of 9.5 of the corrosive solutions, the sample released ferrate ions $\mathrm{FeO}_{4}{ }^{2-}$ into the solutions, but $\mathrm{FeO}_{4}{ }^{2-}$ can oxidize the $\mathrm{Cr}$ of the sample to form $\mathrm{Fe}(\mathrm{OH})_{3}$ and $\mathrm{CrO}_{4}{ }^{2-}$, which could change the color of the solutions to yellow finally [104]. In the meanwhile, the Fe element formed iron hydroxide $\mathrm{Fe}(\mathrm{OH})_{3}$ when reacting with the corrosive solutions. Therefore, the residuals (corrosion product) left on the corroded surfaces of 17-4PH stainless steel in the failure tests should be the mixture of major $\mathrm{Cr}_{2} \mathrm{O}_{3}$ and minor $\mathrm{Fe}_{2} \mathrm{O}_{3}$. 


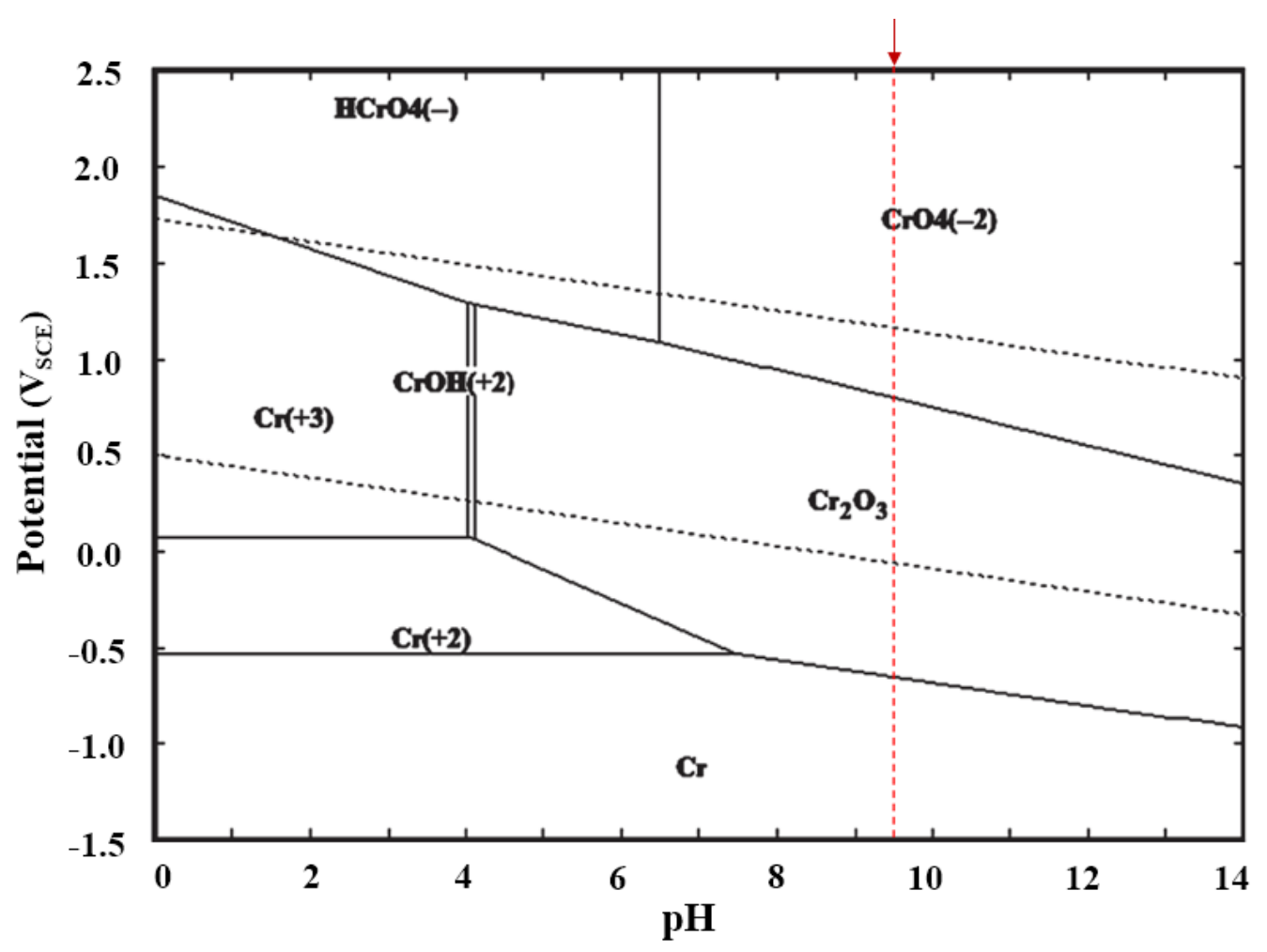

Figure 6-3 Pourbaix diagram for the chromium-water system [99]

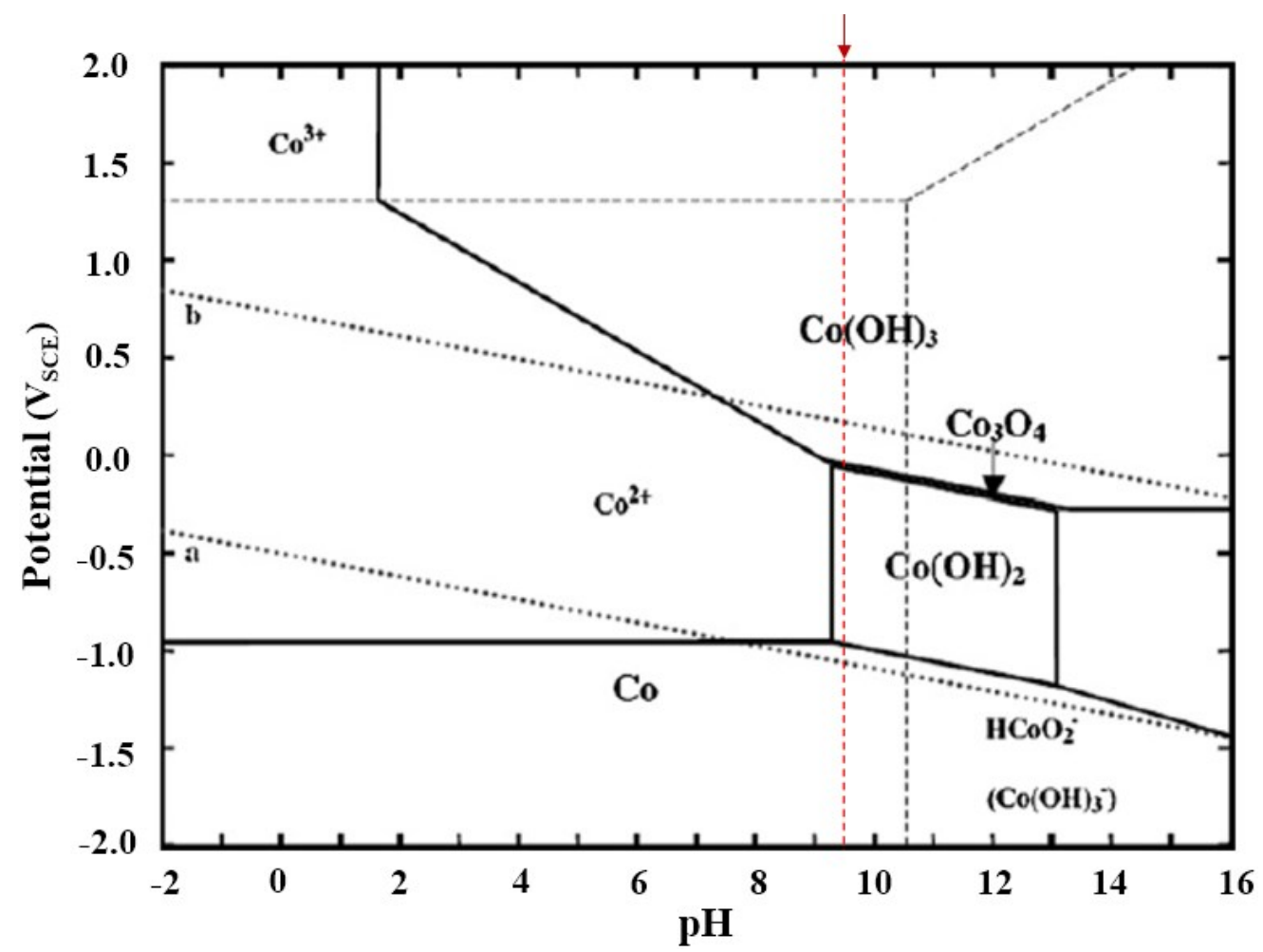

Figure 6-4 Pourbaix diagram for the cobalt-water system [101] 


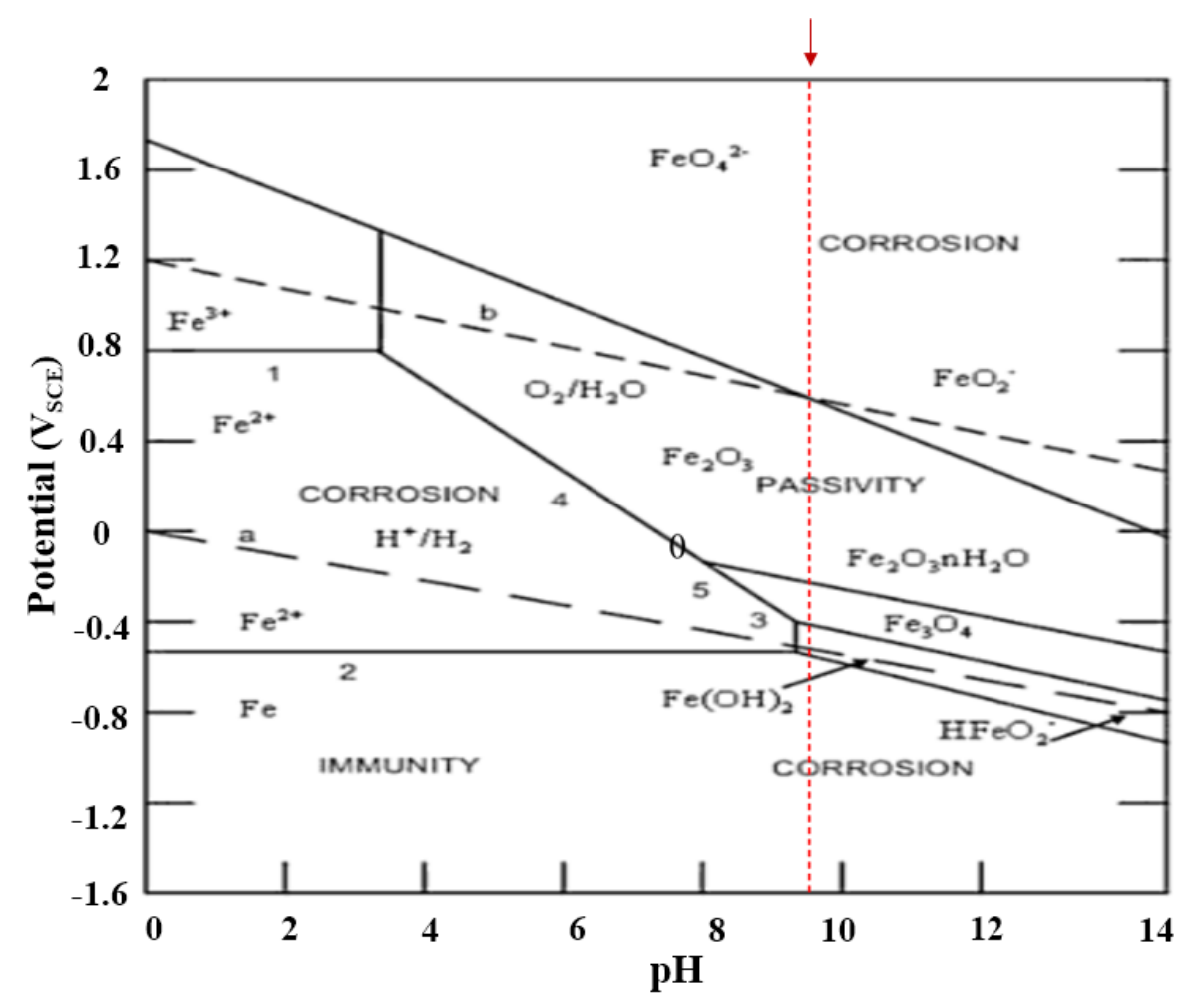

Figure 6-5 Pourbaix diagram for the iron-water system [103]

\subsubsection{Corrosive media}

Although the three solutions were all adjusted to be $\mathrm{pH} 9.5$ in the tests, slight difference in corrosion behavior of Stellite 6 alloy and 17-4PH stainless steel between the solutions was noticed. In general, morpholine exhibited stronger effects on the corrosion of both materials.

Comparing the chemical constituents of the two amine media, morpholine has the chemical formula $\mathrm{O}\left(\mathrm{CH}_{2} \mathrm{CH}_{2}\right)_{2} \mathrm{NH}$. Morpholine has features of both amine and ether functional groups, but there is only one NH linkage because of the heterocycle. Cyclohexylamine has the chemical formula $\mathrm{C}_{6} \mathrm{H}_{11} \mathrm{NH}_{2}$, and there exist two $\mathrm{NH}$ linkages. 
Although the neutralizing amines (morpholine and cyclohexylamine) are different from the filming amines which are known to form a film to act as a barrier between the metal and corrosive constituents in the condensate, they can still attach to the metal surface since they are polar organic compound. These attachments might result from the physical adsorption or the relatively stronger chemisorption [105-108]. The hydrogen bonding was also discovered as a way of adsorption, and a study revealed that the more $\mathrm{N}-\mathrm{H}$ bonds in the amine molecules $[109,110]$, the better corrosion inhibition efficiency of the amine had [111].

Two possible mechanisms of amine adsorption on mild steels are shown in Figure 6-6. Figure 6-6 (a) illustrates the direct chemisorption of the amine on an exposed metal atom through the nitrogen atom, and Figure 6-6 (b) illustrates the hydrogen bond between the amine and the oxidized surface [111]. Cyclohexylamine has a higher number of N-H bonds than morpholine, thus it has more tendency to form hydrogen bonds with the oxidized surface to protect the material.

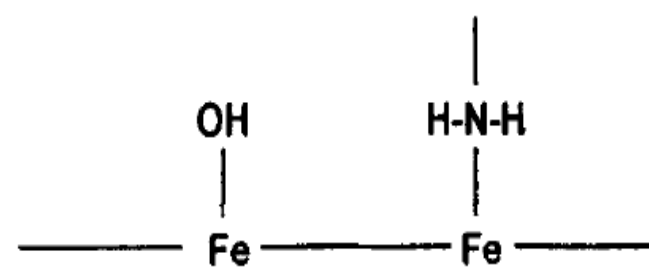

(a)

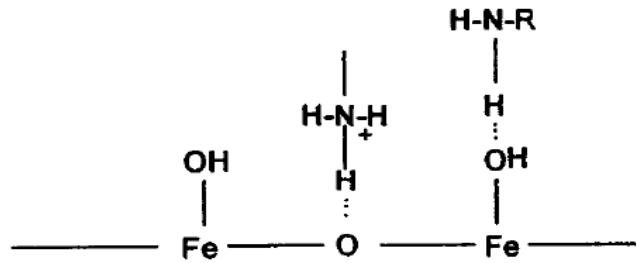

(b)

Figure 6-6 Possible amine adsorption mechanism: (a) chemisorption on the metal surface; (b) hydrogen bonding to the oxidized surface [111]

The sodium hydroxide solution was chosen only for providing the same amount of $\mathrm{OH}^{-}$ ions as for the morpholine and cyclohexylamine solutions at $\mathrm{pH}$ 9.5. In both the primary and failure tests, Stellite 6 alloy and 17-4PH stainless steel exhibited better corrosion 
resistance in sodium hydroxide solution than in morpholine solution, that is, the morpholine acted as a catalyst accelerating the corrosion process.

As for cyclohexylamine solution, the situation is relatively complex. The conclusion could be deduced that cyclohexylamine acted as an inhibitor retarding the corrosion process when accounting for the results of the primary tests. As seen in the current curves of potentiostatic polarization in the failure tests, cyclohexylamine also worked as an inhibitor slowing down the corrosion. Although cyclohexylamine and sodium hydroxide solutions exhibited similar effects on Stellite 6 alloy and 17-4PH stainless steel with respect to corrosion, obvious corrosion product was found on the surfaces of Stellite 6 alloy and 17-4PH stainless steel after the potentiostatic polarization tests in cyclohexylamine solution, but no corrosion product was found on the samples after the potentiostatic polarization tests in sodium hydroxide solution by visual examination. 


\section{Chapter 7: Conclusions and Future Work}

\subsection{Summary}

Stellite 6 alloy and 17-4PH stainless steel are the commonly used materials for boiler feed water service in power plants for trim of valves where amine corrosion occurs. In order to investigate the corrosion behavior of Stellite 6 alloy and 17-4PH stainless steel in amine environments, a series of electrochemical corrosion tests such as potentiodynamic polarization, cyclic polarization, and EIS were conducted on these materials in morpholine, cyclohexylamine and sodium hydroxide solutions at $\mathrm{pH} 9.5$, which simplified the real industrial amine environments. To maintain the test consistence, the $\mathrm{pH}$ compensation system was created for morpholine solution to make the $\mathrm{pH}$ level stable. At the same $\mathrm{pH}$ 9.5 of morpholine, cyclohexylamine and sodium hydroxide solutions, polarization curves and EIS curves were obtained; the corresponding polarization parameters and electrochemical corrosion parameters were calculated or fitted. However, since there was no visual damage on the surfaces of samples in the normal polarization tests, failure polarization tests under a constant higher potential $(4 \mathrm{~V})$ were performed on Stellite 6 alloy and 17-4PH stainless steel in morpholine, cyclohexylamine and sodium hydroxide solutions to generate corrosion damage of the materials. The variations of current with time were recorded in the failure tests, and the corroded surfaces of the samples were examined using SEM/EDX. The experimental results were analyzed and discussed; the corrosion mechanisms were explored. 


\subsection{Conclusions}

The following conclusions can be drawn from the experimental results and associated analyses and discussions:

1. Owing to high $\mathrm{Cr}$ content, Stellite 6 alloy and 17-4PH stainless steel are able to form the oxide films on their surfaces in morpholine, cyclohexylamine and sodium hydroxide solutions to protect further corrosion of the materials. Therefore, on their polarization curves, there is a passivation region or tendency to form passivation region, in particular, on the polarization curves of $17-4 \mathrm{PH}$ stainless steel, representing the formation of the oxide film on its surface.

2. Under a constant higher potential (4 V), Stellite 6 alloy and 17-4PH stainless steel surfaces can be corroded in morpholine, cyclohexylamine and sodium hydroxide solutions at $\mathrm{pH} 9.5$ due to damage of the protective oxide film in the failure tests; obvious surface damages such as corrosion product were observed on the surfaces of the samples.

3. Overall, Stellite 6 alloy has better corrosion or oxidation resistance than 17-4PH stainless steel in morpholine, cyclohexylamine and sodium hydroxide solutions at $\mathrm{pH}$ 9.5 in the primary tests under lower potentials applied; the results of potentiodynamic polarization and EIS are consistent.

4. Both Stellite 6 alloy and 17-4PH stainless steel did not exhibit good resistance to pitting corrosion or erosion/corrosion in morpholine, cyclohexylamine and sodium hydroxide solutions at $\mathrm{pH} 9.5$, because the cyclic polarization curves of these materials all do not have an electropositive hysteresis loop, but the former is better than the latter as the 
cyclic polarization curves of $17-4 \mathrm{PH}$ stainless steel have a large electronegative hysteresis loop while those of Stellite 6 alloy not.

5. The oxide films on Stellite 6 alloy and 17-4PH stainless steel behaved differently under the constant higher potential in morpholine, cyclohexylamine and sodium hydroxide solutions at $\mathrm{pH}$ 9.5. The oxide film on the former was etched entirely, while that on the latter was only damaged partially.

6. Based on the Pourbaix diagrams of $\mathrm{Cr}, \mathrm{Co}$, and $\mathrm{Fe}$, the corrosion product of Stellite 6 alloy in morpholine, cyclohexylamine and sodium hydroxide solutions would be mainly $\mathrm{Cr}_{2} \mathrm{O}_{3}, \mathrm{Co}_{3} \mathrm{O}_{4}$ and $\mathrm{Co}(\mathrm{OH})_{3}$; the chromite ions $\mathrm{CrO}_{4}{ }^{2-}$ dissolved into the solutions can change the color of the solutions to yellow. The corrosion product of 17-4PH stainless steel in morpholine, cyclohexylamine and sodium hydroxide solutions would be mainly $\mathrm{Cr}_{2} \mathrm{O}_{3}$ and $\mathrm{Fe}_{2} \mathrm{O}_{3}$; the chromite ions $\mathrm{CrO}_{4}{ }^{2-}$ dissolved into the solutions can change the color of the solutions to yellow.

7. Morpholine, cyclohexylamine and sodium hydroxide are all alkaline, and their solutions were adjusted to be $\mathrm{pH} 9.5$ in the electrochemical tests. Generally, no significant difference in the test results was found between the solutions, but on the whole, morpholine exhibited a stronger effect on the corrosion of both materials.

\subsection{Significant contributions}

Stellite 6 alloy and 17-4PH stainless steel have been used as the materials for boiler feed water service in power plants for a long time, but the corrosion behavior of these materials in amine media, which are the industrial environments of such applications, has rarely been 
studied. This thesis is the first study of the corrosion behavior of Stellite alloys in amine environments, which has found that Stellite 6 alloy and 17-4PH stainless steel are generally resistant to corrosion in amine media by forming a Cr-rich oxide film on their surface, but they can be corroded when the oxide film is broken, leading to the damage of the surfaces and the removal of the materials. The oxide film of $17-4 \mathrm{PH}$ stainless steel is more resistant to electron transferring than that of Stellite 6 alloy, thus has better resistance to corrosion. However, the oxide film of 17-4PH stainless steel has less durability and it works well only within a certain potential range. Otherwise, Stellite 6 alloy has better overall corrosion and pitting corrosion resistance than $17-4 \mathrm{PH}$ stainless steel in amine environments. These outcomes provide the power generation industry with scientific evidence for material selection.

\subsection{Future work}

This research is a part of the entire project "Investigation of Corrosion Resistance of Stellite Alloys in Amine Environments"; therefore, the following tasks have been planned toward the completion of the entire project:

1. In addition to Stellite 6, other Stellite alloys such as Stellite 21, Stellite 3, Stellite 12, Stellite 712, Stellite 706, Stellite 728 and Stellite 720 are also employed in the amine environments of power plants. These Stellite alloys will also be studied for corrosion behavior in the same amine media using the same means as for Stellite 6 in this research.

2. Morpholine, cyclohexylamine and sodium hydroxide were selected as the corrosive media for the corrosion study of Stellite 6 alloy and 17-4PH stainless steel in this 
research, as a simplification of real amine environments. Although the morpholine, cyclohexylamine and sodium hydroxide solutions were adjusted to be the same $\mathrm{pH} 9.5$, the difference in the test results between the solutions was found, which means that besides $\mathrm{pH}$ level, other factors such as constituents and structures of the corrosive media also play an important role in the corrosion of the materials. Therefore, it is necessary to test the materials in the real amine solutions.

3. Used for the connection components of boiler feed water service in power plants, Stellite alloys are often applied as hardfacing to enhance surface wear resistance. As well known, the microstructure of a material influences its corrosion behavior significantly. In this research, Stellite 6 is a cast product. For the hardfacing application, it is needed to test hardfacing samples of Stellite alloys in amine solutions.

4. Most components of boiler feed water service in power plants operate at elevated temperatures, and as known, temperature influences the corrosion behavior of materials significantly. However, in this thesis, the electrochemical tests were conducted at room temperature as a preliminary study. Further tests of the materials in amine solutions at elevated temperatures will be made in next step of the entire project. 


\section{References}

[1] S. Kapoor, R. Liu, X. Wu, and M. Yao, "Microstructure and wear resistance relations of Stellite alloys," Int. J. Adv. Mater. Sci, vol. 4, pp. 231-248, 2013.

[2] R. Liu, J. Yao, Q. Zhang, M. Yao, and R. Collier, "Microstructures and hardness/wear performance of high-carbon Stellite alloys containing molybdenum," Metallurgical and Materials Transactions A, vol. 46, pp. $5504-5513,2015$.

[3] R. Liu, J. Yao, Q. Zhang, M. X. Yao, and R. Collier, "Effects of molybdenum content on the wear/erosion and corrosion performance of low-carbon Stellite alloys," Materials \& Design, vol. 78, pp. 95-106, 2015.

[4] D. R. Bush, "Be alert to new valve threat: erosion/corrosion in feedwater," Power, vol. 135 , pp. 30-32, 1991.

[5] S. Rishabh. (2016). Presentation on erosion corrosion [Online]. Available: https://www.slideshare.net/RISHABHSHARMA248/presentation-on-erosioncorrosion [Accessed: July 22, 2017]

[6] H. H. Uhlig and R. W. Revie, Corrosion and corrosion control New York city, NY: John Wiley \& Sons, Inc., 1971.

[7] D. Landolt, Corrosion and surface chemistry of metals. Lausanne, CH: EPFL Press, 2007.

[8] R. Kluck, J. Torres, A. Antompietri, and J. Rivera, "Experiences using neutralizing amines to control $\mathrm{pH}$ and minimize FAC in a combined-cycle power plant," Power Plant Chemistry, vol. 13, p. 98, 2011. 
[9] IEA. (2007). Key world energy statistics [Online]. Available:

http://coprocem.org/documents/key_stats_2007.pdf [Accessed: June 19, 2017]

[10] G. Kaushal, H. Singh, and S. Prakash, "Performance of detonation gun-sprayed Ni-20Cr coating on ASTM A213 TP347H steel in a boiler environment," Journal of thermal spray technology, vol. 21, pp. 975-986, 2012.

[11] J. Kitto and S. Stultz, Steam: its generation and use. Barberton, OH: The Babcock \& Wilcox Company, 2005.

[12] J. S. Association, "Water conditioning for boiler feed water and boiler water," JIS B, vol. 8223, 2006.

[13] L. Drbal, K. Westra, and P. Boston, Power plant engineering. Norwell, MA: Kluwer Academic Publisher, 2012.

[14] S. Kenny and D. Pope, "Basic water treatment of steam boilers," Chemco Water Technology, Vancouver, WA, Report, 2000.

[15] B. Miller, J. Munoz, and F. Wiesler, "Boiler feed water degasification using membrane contactors - new methods for optimized performance," presented at the International Water Conference, Orlando, FL, 2005.

[16] NCDENR. (2004). Benefits of chemical treatments [Online]. Available: http://actchemicals.com/Attach/NCDENR_Boiler\%20Chemicals\%20101_31321.p df [Accessed: June 19, 2017]

[17] C. Frayne, Boiler water treatment: principles and practice vol. 2. Los Angeles, CA: Chemical Publishing Company, 2002.

[18] A. U. Malik, S. A. Al Fozan, and M. Al Romiahl, "Relevance of corrosion research in the material selection for desalination plants," presented at the Second Scientific 
Symposium on Maintenance Planning and Operations, King Saud University, Riyadh, KSA, 1993.

[19] S. Tsubakizaki, M. Takada, T. Suto, H. Kawashima, T. Ichihara, and A. Yoshida, "Achievement on OT (Oxygenated Feed-Water Treatment) application and introduction of countermeasures for powdered scale deposit," Mitsubishi Heavy Industries Technical Review, vol. 49, p. 24, 2012.

[20] B. R. Carey. (1959). Boiler water treatment and condensate return line protection [Online]. Available:

https://ir.library.oregonstate.edu/xmlui/bitstream/handle/1957/6088/Boiler_Water _ocr.pdf?sequence=1 [Accessed: June 19, 2017]

[21] A. H. Tuthill and A. P. Castillo. (1999). Selecting correct valve/pump depends on right match of alloy and application [Online]. Available:

http://nipera.org/ /Media/Files/TechnicalLiterature/SelectingCorrectValvesandPu mpsDependonRightMatchofAlloyandApplication_14051_.pdf [Accessed: June $19,2017]$

[22] D. Casada, "Throttled valve cavitation and erosion," Nuclear Plant Aging Program Oak Ridge National Laboratory, Oak Ridge, TN, Manuscript ORNL/NRC/LTR-91/25, 1991.

[23] X. Zhang, "Investigation of corrosion behavior of wrought Stellite alloys," M.A.Sc. thesis, Mechnical Engineering, Carleton University, Ottawa, ON, CA, 2012.

[24] K. C. Antony, "Wear-resistant cobalt-base alloys," JOM, vol. 35, pp. 52-60, 1983.

[25] J. R. Davis, Nickel, cobalt, and their alloys. Materials Park, OH: ASM International, 2000. 
[26] C. Sullivan, M. Donachie, and F. Morral, Centre d'information du cobalt. Brussels, 1970.

[27] A. Frenk and W. Kurz, "Microstructural effects on the sliding wear resistance of a cobalt-based alloy," Wear, vol. 174, pp. 81-91, 1994.

[28] N. McIntyre, D. Zetaruk, and E. Murphy, "X-ray photoelectron spectroscopic study of the aqueous oxidation of stellite-6 alloy," Surface and Interface Analysis, vol. 1, pp. 105-110, 1979.

[29] W. Hocking, F. Stanchell, E. McAlpine, and D. Lister, "Mechanisms of corrosion of stellite-6 in lithiated high temperature water," Corrosion science, vol. 25, pp. $531-557,1985$.

[30] H. So, C. Chen, and Y. Chen, "Wear behaviours of laser-clad stellite alloy 6," Wear, vol. 192, pp. 78-84, 1996.

[31] V. Kuzucu, M. Ceylan, H. Celik, and I. Aksoy, "Microstructure and phase analyses of Stellite 6 plus 6 wt.\% Mo alloy," Journal of Materials Processing Technology, vol. 69, pp. 257-263, 1997.

[32] V. Kuzucu, M. Ceylan, H. Celik, and I. Aksoy, "An investigation of stellite-6 alloy containing $5.0 \mathrm{wt} \%$ silicon," Journal of Materials Processing Technology, vol. 79, pp. 47-51, 1998.

[33] U. Malayoglu and A. Neville, "Comparing the performance of HIPed and cast Stellite 6 alloy in liquid-solid slurries," Wear, vol. 255, pp. 181-194, 2003.

[34] U. Malayoglu and A. Neville, "Mo and W as alloying elements in Co-based alloys - their effects on erosion-corrosion resistance," Wear, vol. 259, pp. 219-229, 2005. 
[35] U. Malayoglu, A. Neville, and H. Lovelock, "Assessing the kinetics and mechanisms of corrosion of cast and HIPed Stellite 6 in aqueous saline environments," Corrosion Science, vol. 47, pp. 1911-1931, 2005.

[36] M. Yao, J. Wu, and Y. Xie, "Wear, corrosion and cracking resistance of some W or Mo-containing Stellite hardfacing alloys," Materials Science and Engineering: A, vol. 407, pp. 234-244, 2005.

[37] S. Chang, H. Wu, and C. Chen, "Impact wear resistance of stellite 6 hardfaced valve seats with laser cladding," Materials and Manufacturing processes, vol. 23, pp. 708-713, 2008.

[38] Y. Birol, "Thermal fatigue testing of Inconel 617 and Stellite 6 alloys as potential tooling materials for thixoforming of steels," Materials Science and Engineering: $A$, vol. 527, pp. 1938-1945, 2010.

[39] Y. Birol, "Thermal fatigue testing of Stellite 6-coated hot work tool steel," Materials Science and Engineering: A, vol. 527, pp. 6091-6097, 2010.

[40] A. Gholipour, M. Shamanian, and F. Ashrafizadeh, "Microstructure and wear behavior of stellite 6 cladding on 17-4 PH stainless steel," Journal of Alloys and Compounds, vol. 509, pp. 4905-4909, 2011.

[41] S. Romo, J. Santa, J. Giraldo, and A. Toro, "Cavitation and high-velocity slurry erosion resistance of welded Stellite 6 alloy," Tribology International, vol. 47, pp. $16-24,2012$.

[42] J. H. Reinshagen and J. C. Witsberger, "Properties of precipitation hardening stainless steel processed by conventional powder metallurgy techniques," Advances in Powder Metallurgy and Particulate Materials, vol. 7, pp. 313-323, 1994. 
[43] J. R. Davis, K. Mills, and S. Lampman, Properties and selection: Irons, steels, and high-performance alloys vol. 1. Materials Park, OH: ASM International, 1990.

[44] W. Chien and C. Tsai, "The investigation on the prediction of tool wear and the determination of optimum cutting conditions in machining 17-4PH stainless steel," Journal of Materials Processing Technology, vol. 140, pp. 340-345, 2003.

[45] M. Esfandiari and H. Dong, "The corrosion and corrosion-wear behaviour of plasma nitrided 17-4PH precipitation hardening stainless steel," Surface and Coatings Technology, vol. 202, pp. 466-478, 2007.

[46] H. J. Rack and D. Kalish, "The strength, fracture toughness, and low cycle fatigue behavior of 17-4 PH stainless steel," Metallurgical Transactions, vol. 5, pp. 1595-1605, 1974.

[47] U. Viswanathan, S. Banerjee, and R. Krishnan, "Effects of aging on the microstructure of 17-4 PH stainless steel," Materials Science and Engineering: A, vol. 104, pp. 181-189, 1988.

[48] U. Viswanathan, P. Nayar, and R. Krishnan, "Kinetics of precipitation in 17-4 PH stainless steel," Materials science and technology, vol. 5, pp. 346-349, 1989.

[49] M. Murayama, K. Hono, and Y. Katayama, "Microstructural evolution in a 17-4 PH stainless steel after aging at $400 \mathrm{C}, "$ Metallurgical and Materials Transactions A, vol. 30, pp. 345-353, 1999.

[50] C. Hsiao, C. Chiou, and J. Yang, "Aging reactions in a 17-4 PH stainless steel," Materials Chemistry and Physics, vol. 74, pp. 134-142, 2002.

[51] P. Kochmański and J. Nowacki, "Activated gas nitriding of 17-4 PH stainless steel," Surface and Coatings Technology, vol. 200, pp. 6558-6562, 2006. 
[52] L. Ping, Q. Cai, B. Wei, and X. Zhang, "Effect of aging temperature on erosion-corrosion behavior of $17-4 \mathrm{PH}$ stainless steels in dilute sulphuric acid slurry," Journal of Iron and Steel Research, International, vol. 13, pp. 73-78, 2006.

[53] J. Bressan, D. Daros, A. Sokolowski, R. Mesquita, and C. Barbosa, "Influence of hardness on the wear resistance of 17-4 $\mathrm{PH}$ stainless steel evaluated by the pin-on-disc testing," Journal of Materials Processing Technology, vol. 205, pp. 353-359, 2008.

[54] H. Mirzadeh, A. Najafizadeh, and M. Moazeny, "Flow curve analysis of 17-4 PH stainless steel under hot compression test," Metallurgical and Materials Transactions A, vol. 40, pp. 2950-2958, 2009.

[55] H. Mirzadeh and A. Najafizadeh, "Aging kinetics of 17-4 PH stainless steel," Materials chemistry and physics, vol. 116, pp. 119-124, 2009.

[56] L. E. Murr, E. Martinez, J. Hernandez, S. Collins, K. N. Amato, S. M. Gaytan, et al., "Microstructures and properties of 17-4 $\mathrm{PH}$ stainless steel fabricated by selective laser melting," Journal of Materials Research and Technology, vol. 1, pp. 167-177, 2012.

[57] H. Mirzadeh and A. Najafizadeh, "Hot deformation and dynamic recrystallization of 17-4 PH stainless steel," ISIJ international, vol. 53, pp. 680-689, 2013.

[58] D. Nakhaie and M. H. Moayed, "Pitting corrosion of cold rolled solution treated 17-4 PH stainless steel," Corrosion Science, vol. 80, pp. 290-298, 2014.

[59] O. Farghaly, R. A. Hameed, and A. A. H. Abu Nawwas, "Analytical application using modern electrochemical techniques," Int. J. Electrochem. Sci, vol. 9, pp. 3287-3318, 2014. 
[60] C. L. Reedy, R. A. Corbett, and M. Burke, "Electrochemical tests as alternatives to current methods for assessing effects of exhibition materials on metal artifacts," Studies in Conservation, vol. 43, pp. 183-196, 1998.

[61] J. R. Scully, "Electrochemical methods for laboratory corrosion testing," in Corrosion Testing and Evaluation: Silver Anniversary Volume, Philadelphia, PA: ASTM International, 1990.

[62] "Basics of electrochemical impedance spectroscopy," Gamry Instrument, Warminster, PA, Application notes, 2007.

[63] N. Cogger and N. Evans, "An introduction to electrochemical impedance measurement," Solarrton analytical, Farnborough, Hampshire, UK, Technical Report 6, 2009.

[64] D. Enos and L. Scribner, "The potentiodynamic polarization scan," Solartron analytical, Farnborough, Hampshire, UK, Technical Report 33, 1997.

[65] "Standard Practice for Calculation of Corrosion Rates and Related Information from Electrochemical Measurements," ASTM Standard G102, 2015.

[66] P. Hu, "Improvement in metal-on-metal bearing of hip implants with advanced Stellite alloys," M.A.Sc. thesis, Materials Engineering, Carleton University, Ottawa, ON, CA, 2013.

[67] J. R. Davis, Corrosion: understanding the basics. Materials Park, OH: ASM International, 2000.

[68] C. Cao and J. Zhang, An introduction to electrochemical impedance spectroscopy vol. 21. Beijing: Science Press, 2002. 
[69] A. Kocijan, D. K. Merl, and M. Jenko, "The corrosion behaviour of austenitic and duplex stainless steels in artificial saliva with the addition of fluoride," Corrosion Science, vol. 53, pp. 776-783, 2011.

[70] S. L. d. Assis and I. Costa, "The effect of polarisation on the electrochemical behavior of Ti-13Nb-13Zr alloy," Materials Research, vol. 10, pp. 293-296, 2007.

[71] A. Popova, E. Sokolova, S. Raicheva, and M. Christov, "AC and DC study of the temperature effect on mild steel corrosion in acid media in the presence of benzimidazole derivatives," Corrosion Science, vol. 45, pp. 33-58, 2003.

[72] J. Qiu, W. Q. Yu, F. Q. Zhang, R. J. Smales, Y. L. Zhang, and C. H. Lu, "Corrosion behaviour and surface analysis of a $\mathrm{Co}-\mathrm{Cr}$ and two $\mathrm{Ni}-\mathrm{Cr}$ dental alloys before and after simulated porcelain firing," European journal of oral sciences, vol. 119, pp. 93-101, 2011.

[73] Y. Zheng, S. Luo, and W. Ke, "Effect of passivity on electrochemical corrosion behavior of alloys during cavitation in aqueous solutions," Wear, vol. 262, pp. 1308-1314, 2007.

[74] L. Faget and M. Rouse, "The effect of silicon on the electrochemical corrosion resistance of carbon steel," California Polytechnic State University, San Luis Obispo, CA, Project report, 2014.

[75] P. Rodič and I. Milošev, "Corrosion inhibition of pure aluminium and alloys AA2024-T3 and AA7075-T6 by Cerium (III) and Cerium (IV) Salts," Journal of The Electrochemical Society, vol. 163, pp. C85-C93, 2016.

[76] M. D. Roach, J. T. Wolan, D. E. Parsell, and J. D. Bumgardner, "Use of X-ray photoelectron spectroscopy and cyclic polarization to evaluate the corrosion 
behavior of six nickel-chromium alloys before and after porcelain-fused-to-metal firing," The Journal of prosthetic dentistry, vol. 84, pp. 623-634, 2000.

[77] F. Blin, P. Koutsoukos, P. Klepetsianis, and M. Forsyth, "The corrosion inhibition mechanism of new rare earth cinnamate compounds - electrochemical studies," Electrochimica acta, vol. 52, pp. 6212-6220, 2007.

[78] A. Chaudhry, R. Bhola, V. Mittal, and B. Mishra, "Ni0.5Zn0.5Fe2O4 as a potential corrosion inhibitor for API 5L X80 steel in acidic environment," International Journal of Electrochemical Science, vol. 9, pp. 4478-4492, 2014.

[79] "Standard test method for conducting potentiodynamic polarization resistance measurements," ASTM Standard G59, 2014.

[80] "Standard reference test method for making potentiodynamic anodic polarization measurements," ASTM Standard G5, 2014.

[81] O. Hollander, "Boiler Systems," in AWT Technical Reference and Training Manual, 2nd ed: Association of Water Technologies, 2015, pp. 58-95.

[82] NCBI. Morpholine [Online]. Available: https://pubchem.ncbi.nlm.nih.gov/compound/8083 [Accessed: June 20, 2017]

[83] H. Hall Jr, "Correlation of the base strengths of amines," Journal of the American Chemical Society, vol. 79, pp. 5441-5444, 1957.

[84] DEAN-ZCF-U. (1986). Use of diethylaminoethanol, morholine, and cyclohexylamine for condensate returen line corrosion prevention [Online]. Available: http://www.dtic.mil/dtic/tr/fulltext/u2/a168297.pdf [Accessed: June 19, 2017] 
[85] NCBI. Cyclohexylamine [Online]. Available:

https://pubchem.ncbi.nlm.nih.gov/compound/7965 [Accessed: June 20, 2017]

[86] NCBI. Sodium hydroxide [Online]. Available:

https://pubchem.ncbi.nlm.nih.gov/compound/14798 [Accessed: June 20, 2017]

[87] K. Popov, L. H. Lajunen, A. Popov, H. Rönkkömäki, M. Hannu-Kuure, and A. Vendilo, "7Li, 23Na, 39K and 133CsNMR comparative equilibrium study of alkali metal cation hydroxide complexes in aqueous solutions. First numerical value for CsOH formation," Inorganic chemistry communications, vol. 5, pp. 223-225, 2002.

[88] E. Guggenheim, "The theoretical basis of Raoult's law," Transactions of the Faraday Society, vol. 33, pp. 151-156, 1937.

[89] S. Ćatić, E. Obralić, and A. Bratovčić, "Rosemary as ecologically acceptable corrosion inhibitor of steel," Bulletin of the Chemists and Technologists of Bosnia and Herzegovina, vol. 46, p. 4, 2016.

[90] M. G. Fontana, Corrosion engineering: Tata McGraw-Hill Education, 2005.

[91] R. G. Kelly, J. R. Scully, D. Shoesmith, and R. G. Buchheit, "Electrochemical thermodynamics and kinetics of relevance to corrosion," in Electrochemical Techniques in Corrosion Science and Engineering. vol. 18, New York, NY: Narcek Dekker, Inc., 2002, pp. 44-55.

[92] E. L. Anderson and P. Bühlmann, "Electrochemical impedance spectroscopy of ion-selective membranes: artifacts in two-, three-, and four-electrode measurements," Analytical Chemistry, vol. 88, pp. 9738-9745, 2016. 
[93] "Standard test method for conducting cyclic potentiodynamic polarization measurements for localized corrosion susceptibility of iron-, nickel-, or cobaltcased alloys," ASTM Standard G61, 2014.

[94] M. Popa, C. Vasilescu, S. I. Drob, P. Osiceanu, M. Anastasescu, and J. M. Calderon-Moreno, "Characterization and corrosion resistance of anodic electrodeposited titanium oxide/phosphate films on Ti-20Nb-10Zr-5Ta bioalloy," Journal of the Brazilian Chemical Society, vol. 24, pp. 1123-1134, 2013.

[95] L. Gu, S. Liu, H. Zhao, and H. Yu, "Facile preparation of water-dispersible graphene sheets stabilized by carboxylated oligoanilines and their anticorrosion coatings," ACS applied materials \& interfaces, vol. 7, pp. 17641-17648, 2015.

[96] G. McRae, M. Maguire, C. Jeffrey, D. Guzonas, and C. Brown, "A comparison of fractal dimensions determined from atomic force microscopy and impedance spectroscopy of anodic oxides on Zr-2.5 Nb," Applied Surface Science, vol. 191, pp. 94-105, 2002.

[97] P. Alnegren, "Oxidation behavior of selected $\mathrm{FeCr}$ alloys in environments relevant for solid oxide electrolysis applications," M.S. thesis, Chemical and Biological Engineering, Chalmers University of Technology, Göteborg, Sweden, 2012.

[98] I. Panas, J. E. Svensson, H. Asteman, T. J. Johnson, and L. G. Johansson, "Chromic acid evaporation upon exposure of $\mathrm{Cr} 2 \mathrm{O} 3$ (s) to $\mathrm{H} 2 \mathrm{O}$ (g) and $\mathrm{O} 2$ (g)-mechanism from first principles," Chemical Physics Letters, vol. 383, pp. 549-554, 2004.

[99] D. Bregnbak, J. D. Johansen, M. S. Jellesen, C. Zachariae, T. Menné, and J. P. Thyssen, "Chromium allergy and dermatitis: prevalence and main findings," Contact dermatitis, vol. 73, pp. 261-280, 2015. 
[100] V. Marimuthu, I. Dulac, and K. Kannoorpatti, "Significance of Pourbaix diagrams to study the corrosion behaviour of hardfacing alloys based on chromium carbides at 298 K (25 C)," Journal of Bio-and Tribo-Corrosion, vol. 2, pp. 1-7, 2016.

[101] E. Garcia, J. Santos, E. Pereira, and M. Freitas, "Electrodeposition of cobalt from spent Li-ion battery cathodes by the electrochemistry quartz crystal microbalance technique," Journal of Power Sources, vol. 185, pp. 549-553, 2008.

[102] B. Beverskog and I. Puigdomenech, "Revised Pourbaix diagrams for iron at 25-300C," Corrosion Science, vol. 38, pp. 2121-2135, 1996.

[103] V. Nguyen, "Determining localized anode condition to maintain effective corrosion protection," M.S. thesis, Chemical Engineering, Oregon State Univeristy, Corvallis, OR, 2009.

[104] S. Licht and B. Wang, "Nonaqueous phase Fe (VI) electrochemical storage and discharge of super-iron/lithium primary batteries," Electrochemical and Solid-State Letters, vol. 3, pp. 209-212, 2000.

[105] J. Breston, "Corrosion control with organic inhibitors," Industrial \& Engineering Chemistry, vol. 44, pp. 1755-1761, 1952.

[106] N. Hackerman and H. R. Schmidt, "The adsorption of organic corrosion inhibitors on iron and steel surfaces. electron diffraction studies," The Journal of Physical Chemistry, vol. 53, pp. 629-638, 1949.

[107] G. Banerjee and S. Malhotra, "Contribution to adsorption of aromatic amines on mild steel surface from $\mathrm{HCl}$ solutions by impedance, UV, and Raman spectroscopy," Corrosion, vol. 48, pp. 10-15, 1992. 
[108] N. Hackerman and A. Makrides, "Action of polar organic inhibitors in acid dissolution of metals," Industrial \& Engineering Chemistry, vol. 46, pp. 523-527, 1954.

[109] R. D. Braun, E. E. Lopez, and D. P. Vollmer, "Low molecular weight straight-chain amines as corrosion inhibitors," Corrosion science, vol. 34, pp. 1251-1257, 1993.

[110] M. J. Incorvio and S. Contarini, "X-ray photoelectron spectroscopic studies of metal/inhibitor systems: structure and bonding at the iron/amine interface," Journal of the Electrochemical Society, vol. 136, pp. 2493-2498, 1989.

[111] P. Li, J. Lin, K. Tan, and J. Lee, "Electrochemical impedance and X-ray photoelectron spectroscopic studies of the inhibition of mild steel corrosion in acids by cyclohexylamine," Electrochimica Acta, vol. 42, pp. 605-615, 1997. 


\section{Appendices}

The corrosion products of the Stellite 6 alloy sample could be collected using the electrical tape (Figure A1) from the white specimen holder in morpholine solution and in cyclohexylamine solution after potentiostatic polarization for $25000 \mathrm{~s}$, but no corrosion product could be collected using this method after the tests in sodium hydroxide solution. Two different regions of the corrosion products on the Stellite 6 alloy sample tested in morpholine solution could be identified, as shown in Figure A2. It can be seen that region 1 is much larger than region 2. These two regions were analyzed using EDX, the results are reported in Figure A3 and Figure A4. Similarly, two different regions of the corrosion products on the Stellite 6 alloy sample tested in cyclohexylamine solution could be identified, as shown in Figure A5. These two regions were also analyzed using EDX, the results are reported in Figure A6 and Figure A7.

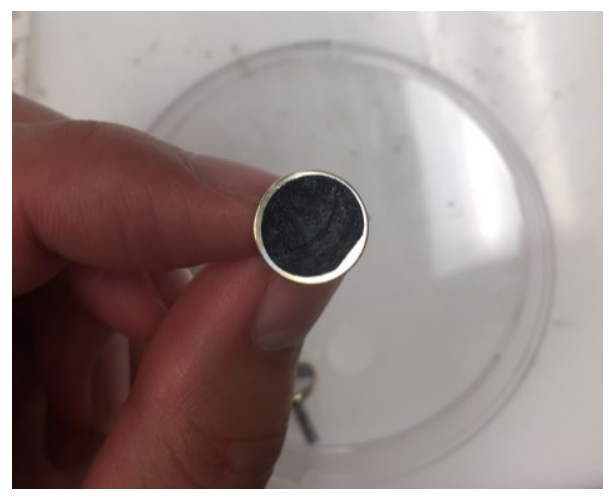

Figure A1 The electrical tape for collecting the corrosion products 


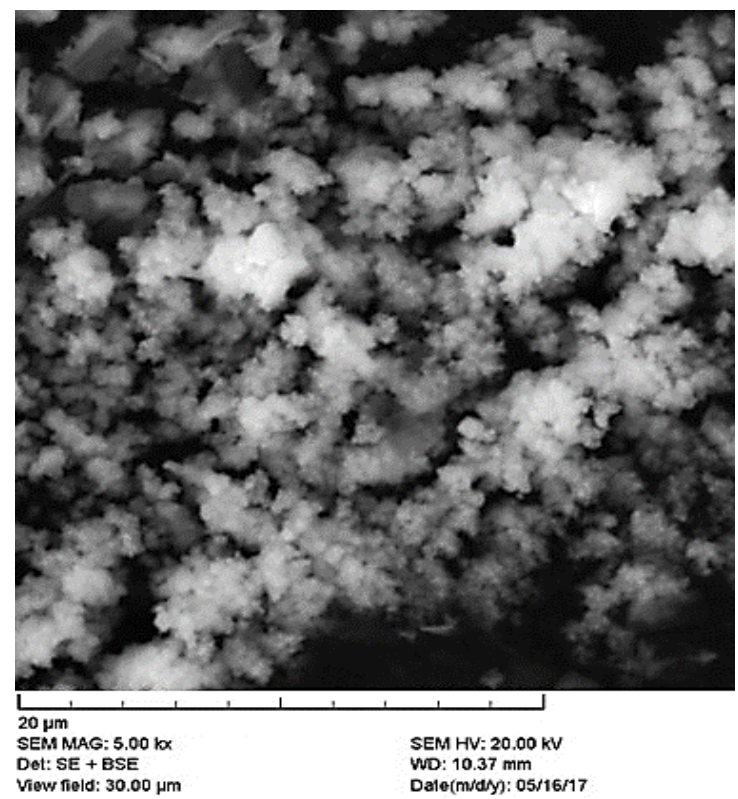

(a)

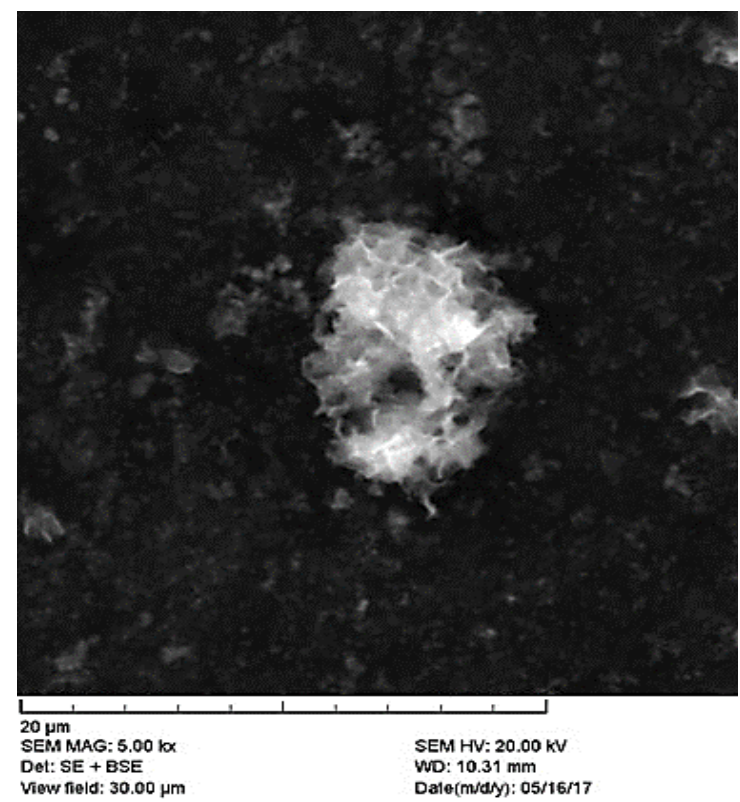

(b)

Figure A2 SEM images of the corrosion products of the Stellite 6 alloy sample tested in morpholine solution: (a) region 1; (b) region 2

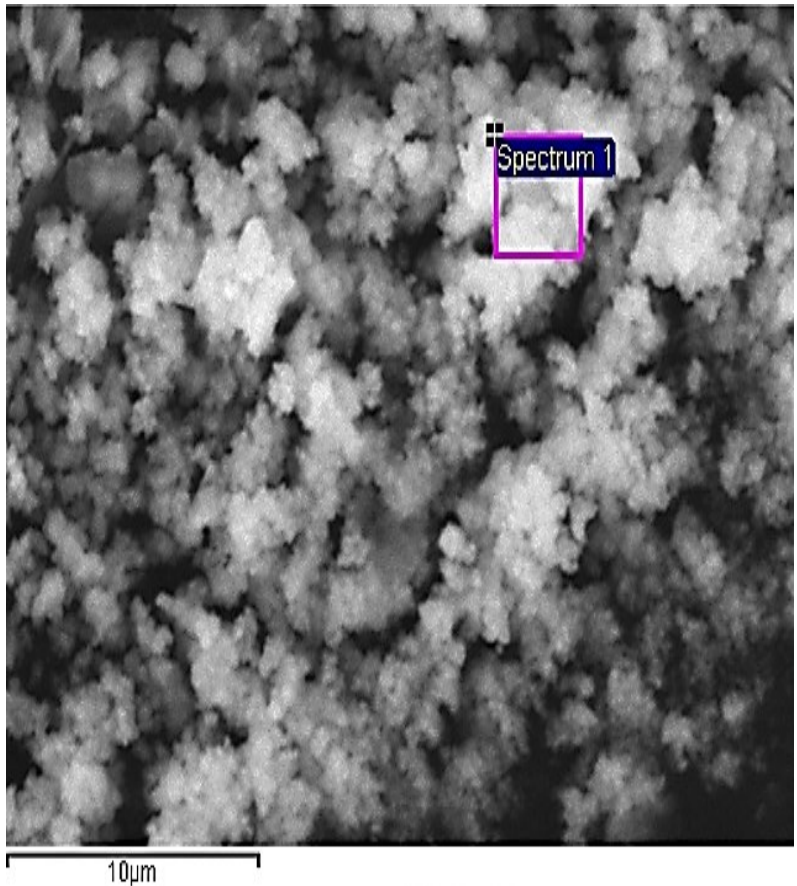

(a)

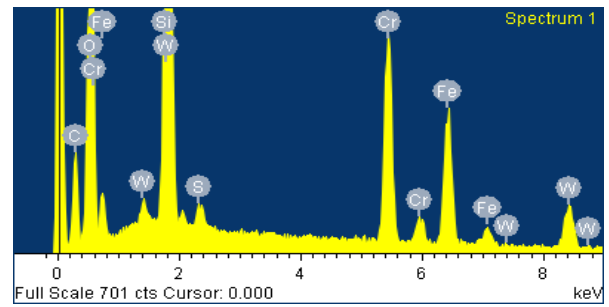

(b)

\begin{tabular}{|c|c|c|}
\hline Element & wt\% & at\% \\
\hline C K & 16.23 & 25.51 \\
\hline O K & 51.20 & 60.43 \\
\hline Si K & 1.86 & 1.25 \\
\hline S K & 0.55 & 0.33 \\
\hline Cr K & 14.49 & 5.26 \\
\hline Fe K & 12.81 & 4.33 \\
\hline W M & 28.10 & 2.89 \\
\hline Totals & 125.23 & 100.00 \\
\hline
\end{tabular}

(c)

Figure A3 EDX results of region 1 of the corrosion products on the Stellite 6 alloy sample tested in morpholine solution: (a) SEM morphology; (b) EDX spectrum;

(c) elemental content table 


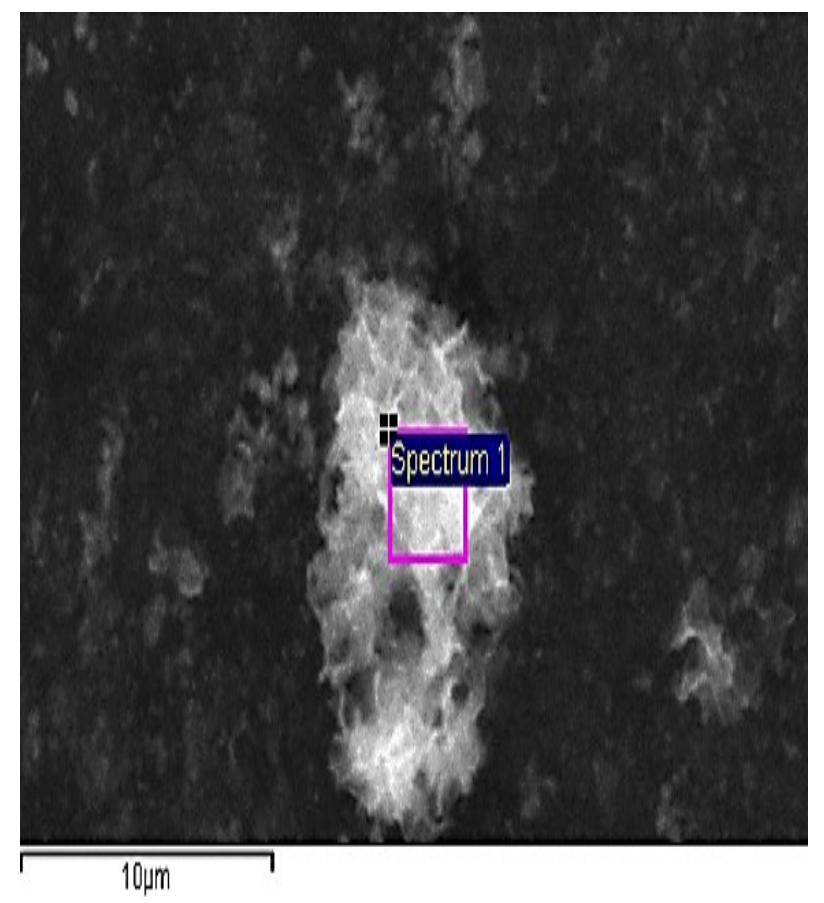

(a)

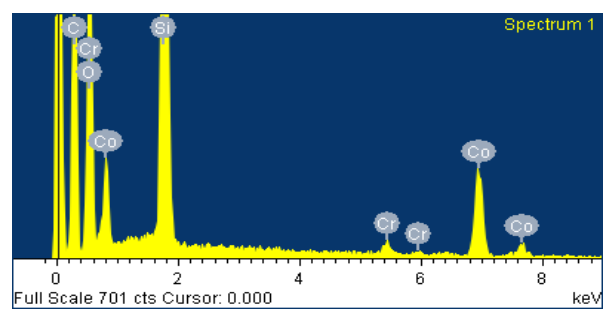

(b)

\begin{tabular}{|c|c|c|}
\hline Element & wt\% & at\% \\
\hline C K & 66.05 & 56.59 \\
\hline O K & 53.25 & 34.25 \\
\hline Si K & 19.34 & 7.09 \\
\hline Cr K & 0.79 & 0.16 \\
\hline Co K & 10.94 & 1.91 \\
\hline Totals & 150.37 & 100.00 \\
\hline
\end{tabular}

(c)

Figure A4 EDX results of region 2 of the corrosion products on the Stellite 6 alloy sample tested in morpholine solution: (a) SEM morphology; (b) EDX spectrum; (c) elemental content table

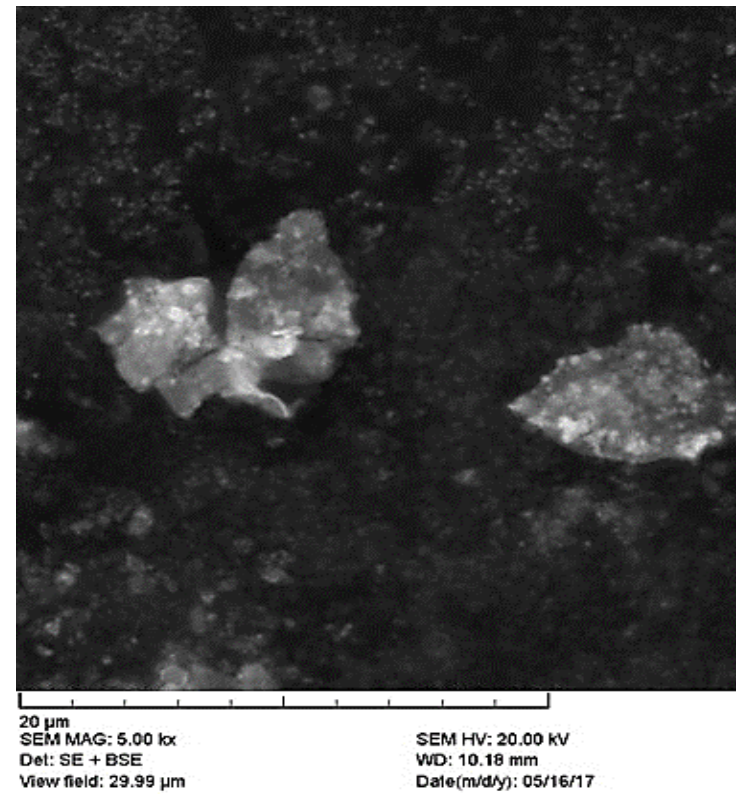

(a)

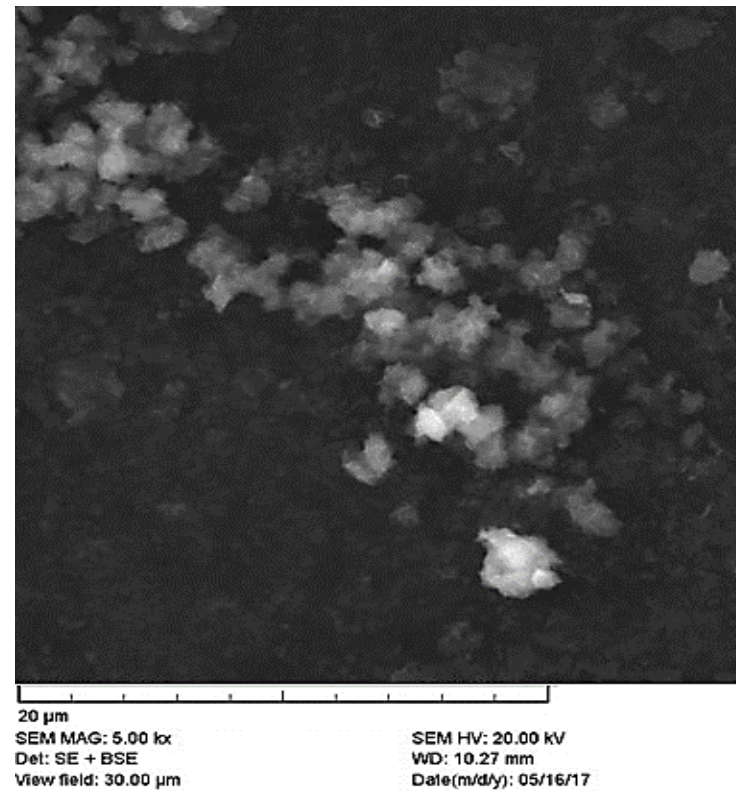

(b)

Figure A5 SEM images of the corrosion products of the Stellite 6 alloy sample tested in cyclohexylamine solution: (a) region 1; (b) region 2 


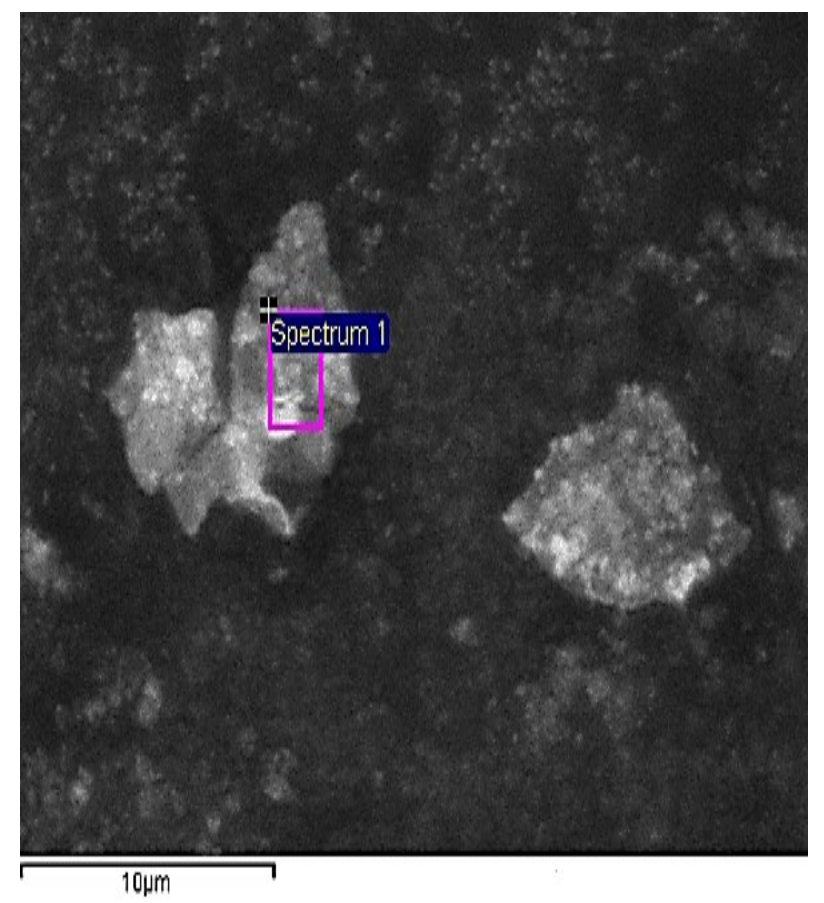

(a)

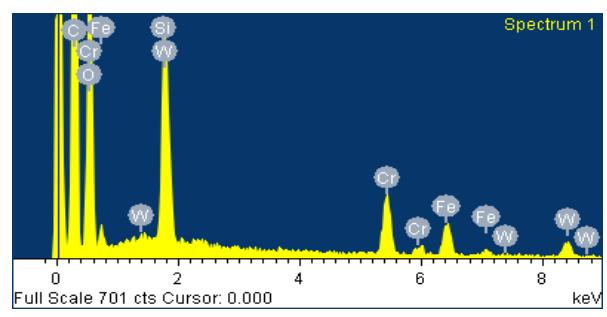

(b)

\begin{tabular}{|c|c|c|}
\hline Element & wt\% & at\% \\
\hline C K & 72.36 & 64.30 \\
\hline O K & 48.83 & 32.57 \\
\hline Si K & 2.98 & 1.13 \\
\hline Cr K & 4.43 & 0.91 \\
\hline Fe K & 3.70 & 0.71 \\
\hline W M & 6.58 & 0.38 \\
\hline Totals & 138.89 & 100.00 \\
\hline
\end{tabular}

(c)

Figure A6 EDX results of region 1 of the corrosion products on the Stellite 6 alloy sample tested in cyclohexylamine solution: (a) SEM morphology; (b) EDX spectrum; (c) elemental content table

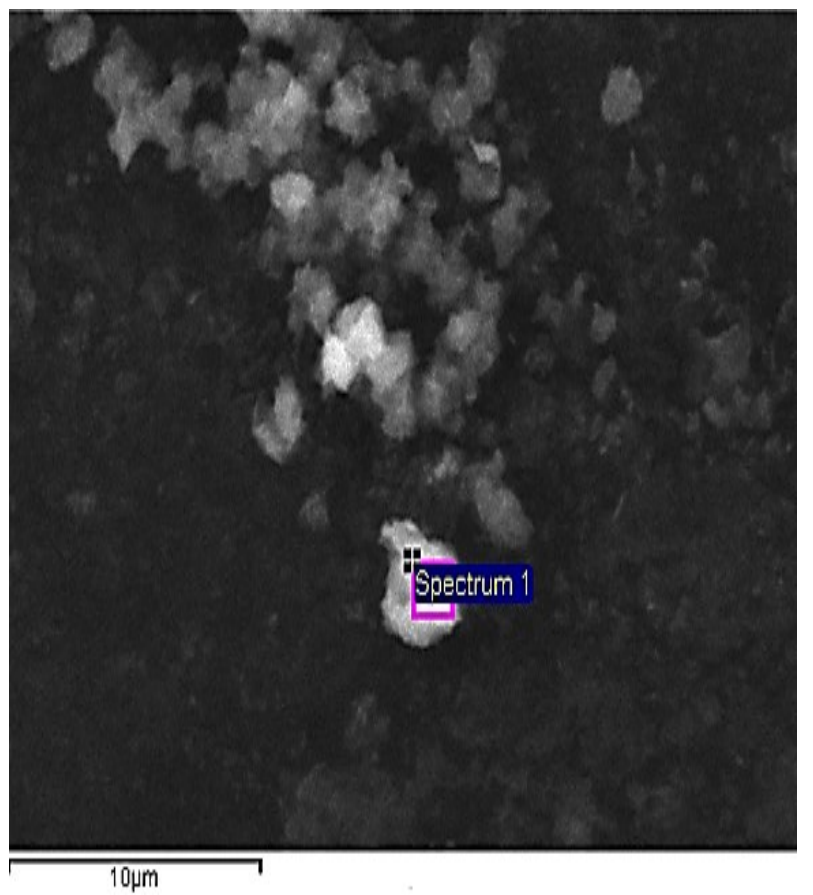

(a)

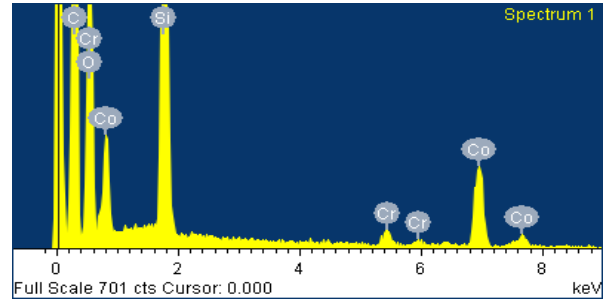

(b)

\begin{tabular}{|c|c|c|}
\hline Element & wt\% & at\% \\
\hline C K & 126.53 & 66.55 \\
\hline O K & 74.07 & 29.25 \\
\hline Si K & 12.82 & 2.88 \\
\hline Cr K & 1.12 & 0.14 \\
\hline Co K & 11.08 & 1.19 \\
\hline Totals & 225.61 & 100.00 \\
\hline
\end{tabular}

Figure A7 EDX results of region 2 of the corrosion products on the Stellite 6 alloy sample tested in cyclohexylamine solution: (a) SEM morphology; (b) EDX spectrum; (c) elemental content table 
The corrosion products on the 17-4PH stainless steel sample in morpholine solution and cyclohexylamine solution after potentiostatic polarization for $1500 \mathrm{~s}$ could be collected using a pipette and then dripped onto a glass slice. Carbon conductive coating was used to enhance the conductivity of the glass slice before the SEM/EDX analyses. No corrosion products were found on 17-4PH stainless steel sample after the tests in sodium hydroxide solution. Two regions of the corrosion products of the 17-4PH stainless steel sample tested in morpholine solution could be identified, as shown in Figure A8. These regions were analyzed with EDX, the results are reported in Figure A9 and Figure A10. For the corrosion products on the 17-4PH stainless steel sample in cyclohexylamine solution, there are almost no distinct regions, but there are some light regions and dim regions that can be identified, as shown in Figure A11. These regions were analyzed with EDX, the results are reported in Figure A12 and Figure A13.

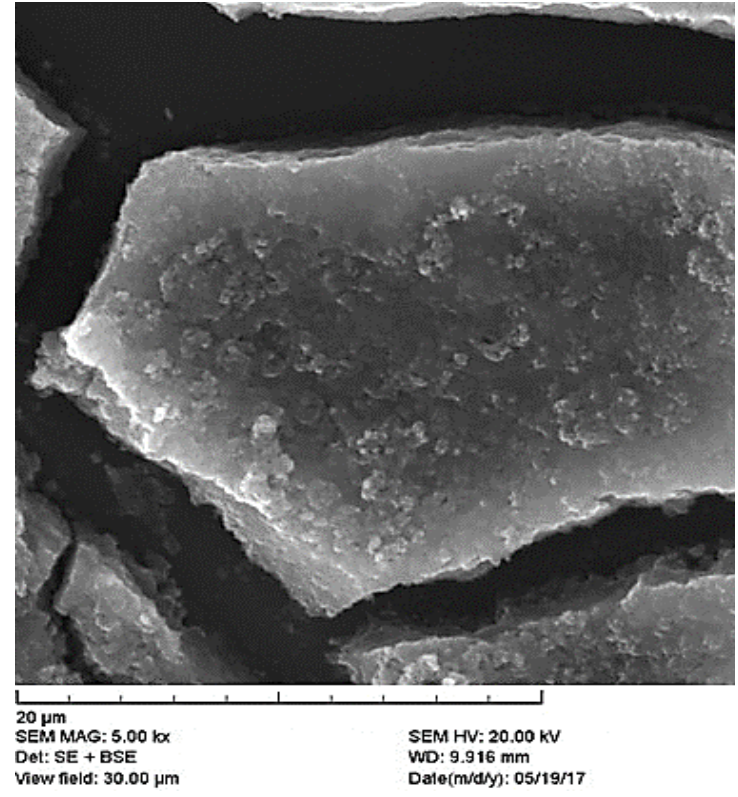

(a)

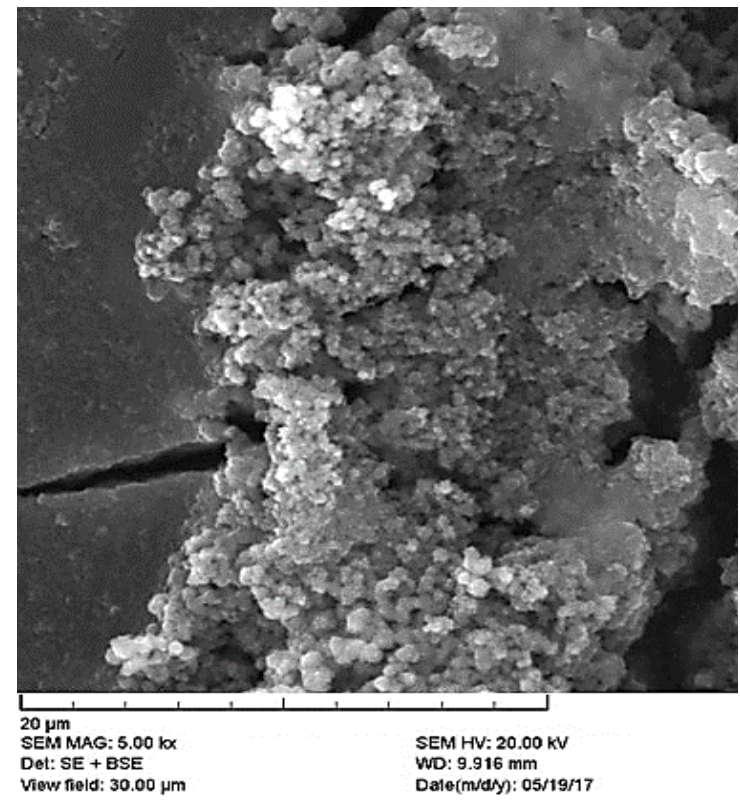

(b)

Figure A8 SEM images of the corrosion products of the 17-4PH stainless steel sample tested in morpholine solution: (a) region 1; (b) region 2 


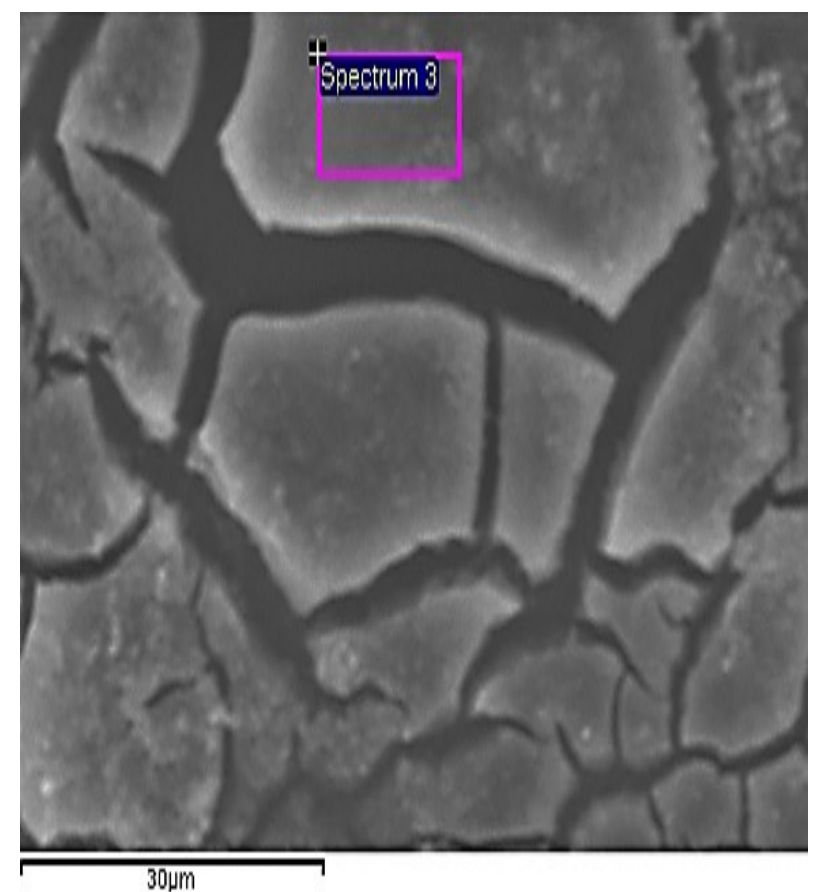

(a)

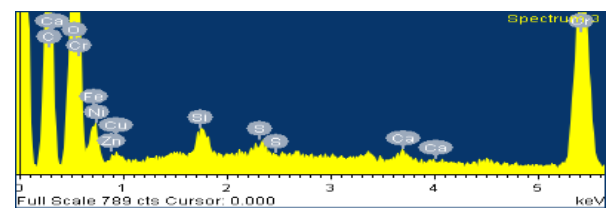

(b)

\begin{tabular}{|c|c|c|}
\hline Element & wt\% & at\% \\
\hline C K & 41.50 & 49.37 \\
\hline O K & 43.26 & 38.64 \\
\hline Si K & 0.74 & 0.38 \\
\hline S K & 0.31 & 0.14 \\
\hline Ca K & 0.33 & 0.12 \\
\hline Cr K & 14.45 & 3.97 \\
\hline Fe K & 25.75 & 6.59 \\
\hline Ni K & 1.09 & 0.27 \\
\hline Cu K & 1.64 & 0.37 \\
\hline Zn K & 0.77 & 0.17 \\
\hline Totals & 129.85 & 100.00 \\
\hline
\end{tabular}

(c)

Figure A9 EDX results of region 1 of the corrosion products on the 17-4PH stainless steel sample tested in morpholine solution: (a) SEM morphology; (b) EDX spectrum; (c) elemental content table

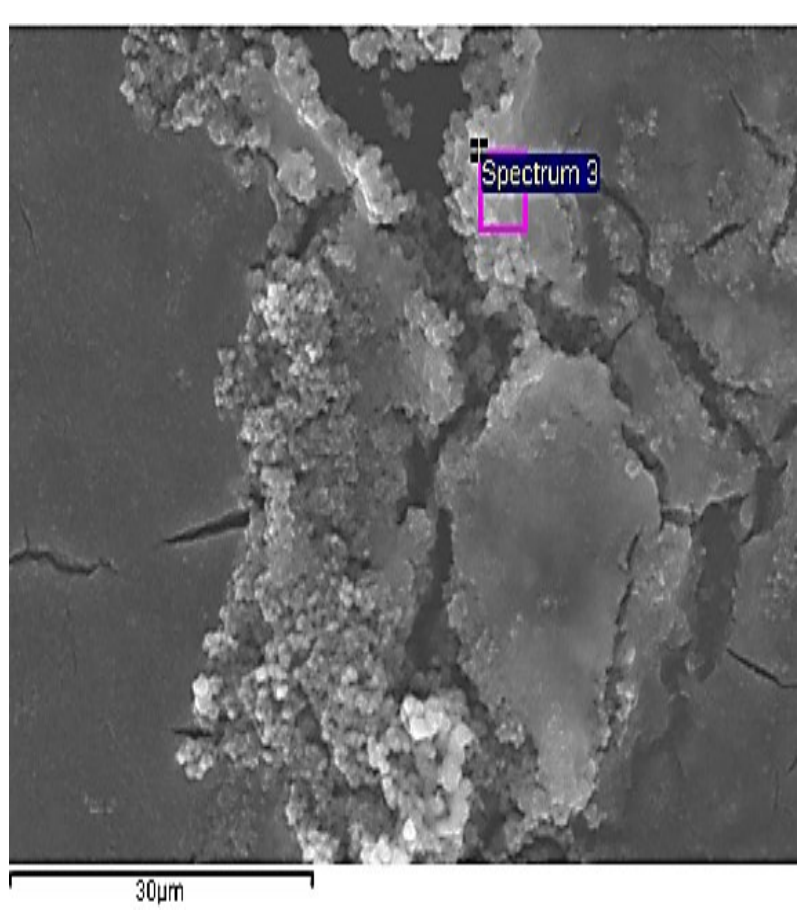

(a)

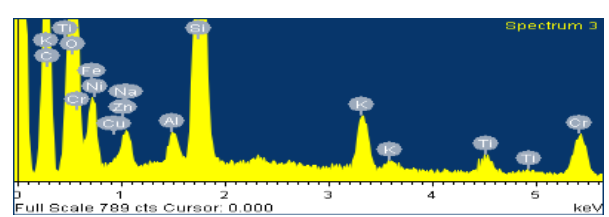

(b)

\begin{tabular}{|c|c|c|}
\hline Element & wt\% & at\% \\
\hline C K & 48.47 & 46.57 \\
\hline O K & 55.48 & 40.02 \\
\hline Na K & 1.50 & 0.75 \\
\hline Al K & 0.90 & 0.38 \\
\hline Si K & 11.10 & 4.56 \\
\hline K K & 2.15 & 0.63 \\
\hline Ti K & 0.95 & 0.23 \\
\hline Cr K & 3.09 & 0.69 \\
\hline Fe K & 26.94 & 5.57 \\
\hline Ni K & 0.74 & 0.15 \\
\hline Cu K & 0.99 & 0.18 \\
\hline Zn K & 1.58 & 0.28 \\
\hline Totals & 153.88 & 100.00 \\
\hline \multicolumn{2}{|c}{} \\
\hline
\end{tabular}

(c)

Figure A10 EDX results of region 2 of the corrosion products on the 17-4PH stainless steel sample tested in morpholine solution: (a) SEM morphology; (b) EDX spectrum; (c) elemental content table 


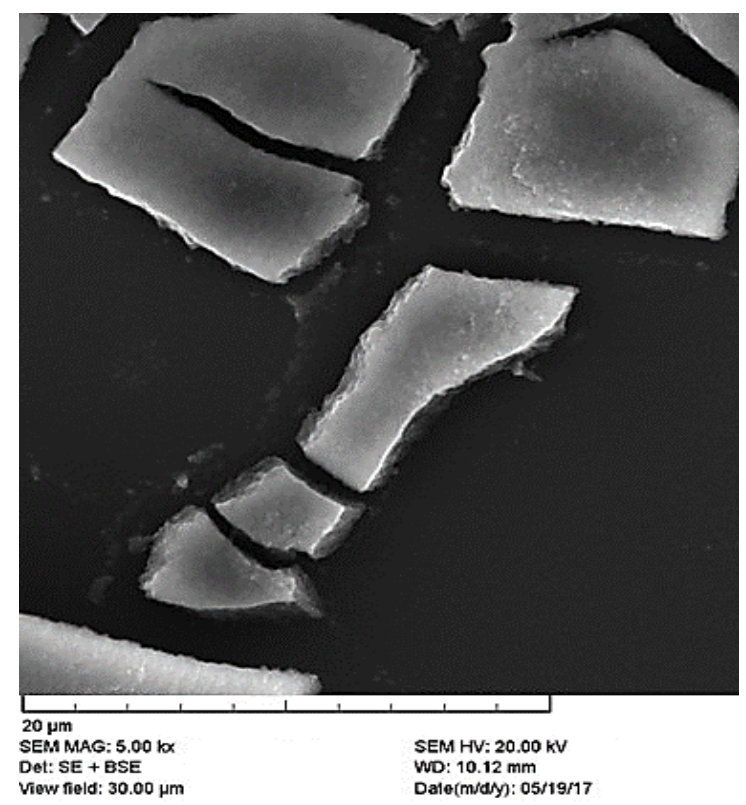

(a)

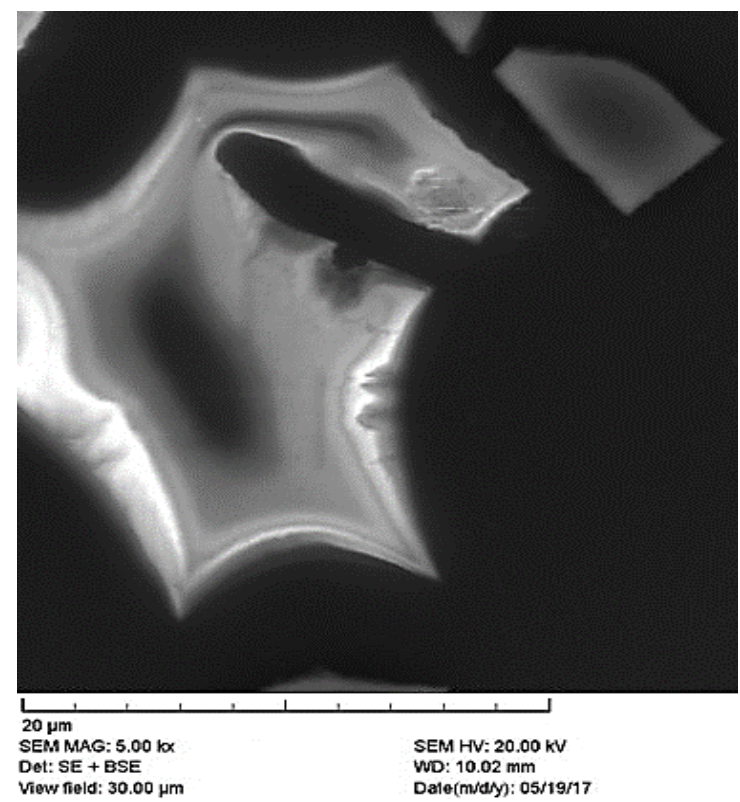

(b)

Figure A11 SEM images of the corrosion products of the 17-4PH stainless steel sample tested in cyclohexylamine solution: (a) region 1; (b) region 2

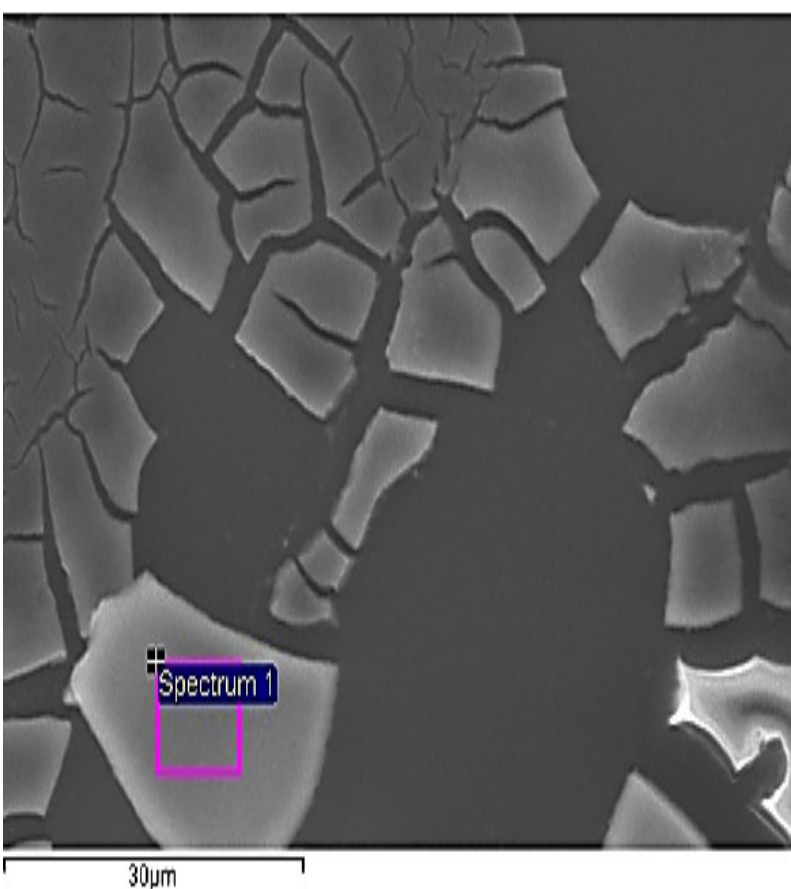

(a)

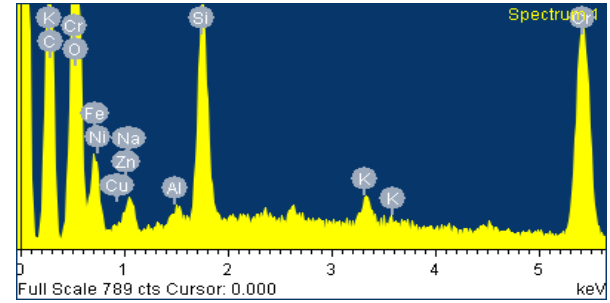

(b)

\begin{tabular}{|c|c|c|}
\hline Element & wt\% & at\% \\
\hline C K & 37.77 & 48.78 \\
\hline O K & 33.73 & 32.70 \\
\hline Na K & 1.18 & 0.79 \\
\hline Al K & 0.30 & 0.17 \\
\hline Si K & 4.09 & 2.26 \\
\hline K K & 0.62 & 0.25 \\
\hline Cr K & 10.27 & 3.06 \\
\hline Fe K & 40.12 & 11.14 \\
\hline Ni K & 0.98 & 0.26 \\
\hline Cu K & 1.69 & 0.41 \\
\hline Zn K & 0.73 & 0.17 \\
\hline Totals & 131.48 & 100.00 \\
\hline
\end{tabular}

(c)

Figure A12 EDX results of region 1 of the corrosion products on the 17-4PH stainless steel sample tested in cyclohexylamine solution: (a) SEM morphology; (b) EDX spectrum; (c) elemental content table 


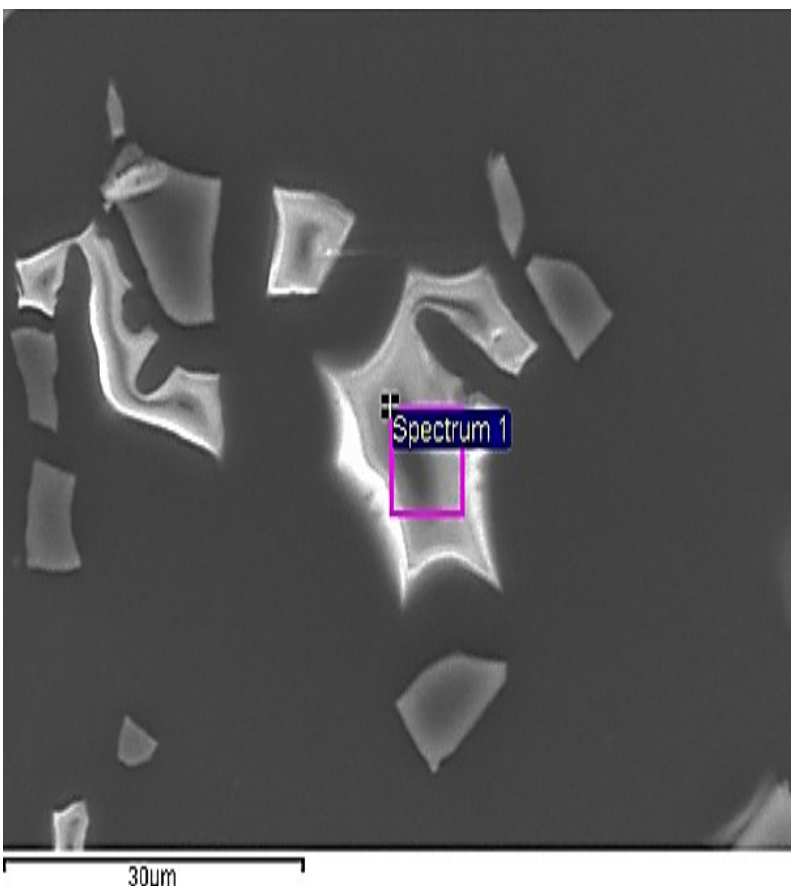

(a)

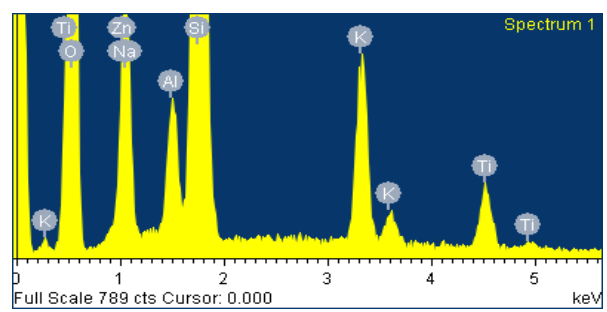

(b)

\begin{tabular}{|c|c|c|}
\hline Element & wt\% & at\% \\
\hline O K & 55.44 & 68.09 \\
\hline Na K & 5.72 & 4.89 \\
\hline Al K & 2.17 & 1.58 \\
\hline Si K & 29.77 & 20.83 \\
\hline K K & 5.15 & 2.59 \\
\hline Ti K & 2.34 & 0.96 \\
\hline Zn K & 3.53 & 1.06 \\
\hline Totals & 104.12 & 100.00 \\
\hline
\end{tabular}

(c)

Figure A13 EDX results of region 2 of the corrosion products on the 17-4PH stainless steel sample tested in cyclohexylamine solution: (a) SEM morphology; (b) EDX spectrum; (c) elemental content table 